\title{
Proteomics investigations : towards mechanisms and biomarkers for drug-induced hepatotoxicity
}

Citation for published version (APA):

Anke Summeren van, A. (2014). Proteomics investigations : towards mechanisms and biomarkers for drug-induced hepatotoxicity. [Doctoral Thesis, Maastricht University]. Maastricht University. https://doi.org/10.26481/dis.20140312as

Document status and date:

Published: 01/01/2014

DOI:

10.26481/dis.20140312as

Document Version:

Publisher's PDF, also known as Version of record

\section{Please check the document version of this publication:}

- A submitted manuscript is the version of the article upon submission and before peer-review. There can be important differences between the submitted version and the official published version of record.

People interested in the research are advised to contact the author for the final version of the publication, or visit the DOI to the publisher's website.

- The final author version and the galley proof are versions of the publication after peer review.

- The final published version features the final layout of the paper including the volume, issue and page numbers.

Link to publication

\footnotetext{
General rights rights.

- You may freely distribute the URL identifying the publication in the public portal. please follow below link for the End User Agreement:

www.umlib.nl/taverne-license

Take down policy

If you believe that this document breaches copyright please contact us at:

repository@maastrichtuniversity.nl

providing details and we will investigate your claim.
}

Copyright and moral rights for the publications made accessible in the public portal are retained by the authors and/or other copyright owners and it is a condition of accessing publications that users recognise and abide by the legal requirements associated with these

- Users may download and print one copy of any publication from the public portal for the purpose of private study or research.

- You may not further distribute the material or use it for any profit-making activity or commercial gain

If the publication is distributed under the terms of Article $25 \mathrm{fa}$ of the Dutch Copyright Act, indicated by the "Taverne" license above, 


\section{Proteomics investigations}

towards mechanisms and biomarkers

for drug-induced hepatotoxicity

Anke Van Summeren 


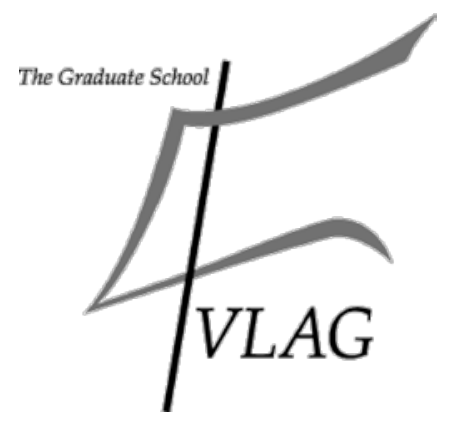

The studies presented in this thesis were performed whitin GROW, school for Oncology and Developmental Biology in collaboration with NUTRIM, school for Nutrition, Toxicology and Metabolism which participates in the Graduate School VLAG (Food Technology, Agrobiotechnology, Nutrition and Health Sciences), accredited by the Royal Netherlands academy of Arts and Sciences.

Layout \& Cover Design: Anke Van Summeren

Printed by: Ridderprint BV, Ridderkerk

CC Copyright Anke Van Summeren, Maastricht 2014

ISBN: 978-90-5335-794-1 


\title{
Proteomics investigations
}

\author{
towards mechanisms and biomarkers \\ for drug-induced hepatotoxicity
}

\section{PROEFSCHRIFT}

ter verkrijging van de graad van doctor aan de Universiteit Maastricht, op gezag van de Rector Magnificus, prof. dr. L. L. G. Soete, volgens het besluit van het College van Decanen, in het openbaar te verdedigen op woensdag 12 maart 2014 om 16:00 uur

$$
\text { door }
$$

\section{Anke Van Summeren}

geboren te Lommel op 17 augustus 1984 


\section{Promotores}

Prof. dr. J. C. S. Kleinjans

Prof. dr. E. C. M. Mariman

\section{Copromotor}

Dr. J. Renes

\section{Beoordelingscommissie}

Prof. dr. W. H. Lamers (voorzitter)

Prof. dr. L. Arckens (Katholieke Universiteit Leuven, België)

Prof. dr. H. van Loveren (RIVM, Bilthoven)

Dr. S. Olde Damink

Prof. dr. H. H. H. W. Schmidt

The research described in this thesis was funded by the Netherlands Genomics Initiative (NGI) from the Netherlands Organization for Scientific Research (NWO), (050-060-510). 
CHAPTER 1

CHAPTER 2

CHAPTER 3

CHAPTER 4

CHAPTER 5

CHAPTER 6

CHAPTER 7

CHAPTER 8

Proteomics investigations of drug-induced hepatotoxicity in HepG2 cells

Screening for drug-induced hepatotoxicity in primary mouse hepatocytes using acetaminophen, amiodarone and cyclosporin A as model compounds: an 'omics-guided approach

The secretome of primary mouse hepatocytes in drug-induced hepatotoxicity screening

Conserved hepatotoxic responses in HepG2 and primary mouse hepatocytes

In vitro to in vivo comparison of cyclosporin A-induced protein expression profiles

Analysis of protein expression patterns induced by classifying hepatotoxicants in primary mouse hepatocytes

Summary and general discussion

Nederlandse samenvatting

Supplemental data

Dankwoord

Curriculum Vitae

List of publications 



\section{CHAPTER 1}

\section{General introduction}

Proteomics in the search for mechanisms and biomarkers of drug-induced hepatotoxicity

Anke Van Summeren, Johan Renes, Joost H. M. van Delft, Jos C. S. Kleinjans and Edwin C. M. Mariman

Toxicology in vitro : an international journal published in association with BIBRA 26, 373-385, (2012). 


\section{INTRODUCTION}

In the development of new medicins the appearance of unexpected toxicity is one of the major reasons for the withdrawal of the product from the market. With respect to toxicity testing hepatotoxicity is most prominent, because after oral intake the compounds will be transported to the liver, where they will be metabolized and eliminated. Currently, hepatotoxicity is evaluated in 28-day in vivo repeated-dose toxicity tests by analysis of hematological, histopathological and clinical parameters. However, these parameters are often insensitive and can generate false negative results ${ }^{1,2}$. Consequently, unexpected hepatotoxicity often appears in the clinical trials or even when the product is already on the market ${ }^{3}$. This emphasizes the need for novel screening methods that address toxicological hazards early in the developmental process ${ }^{4}$. These new screening methods are preferably applied on in vitro test systems to reduce the number of laboratory animals. The 'omics-technologies have already shown promising results for improving the current toxicity tests, in particular transcriptomic based screenings. However, studying the transcription level of a gene only gives a rather rough estimate of its corresponding protein expression level. With proteomics the functional molecules, are investigated which may reveal new biomarkers and toxicity signatures for preclinical safety assessment and disease diagnostics ${ }^{4}$. As such a better understanding of the underlying molecular mechanisms-of-action can be achieved. Proteomics used for predictive or mechanistic toxicological research with acute or long-term exposure to toxicants is known as toxicoproteomics, which was first introduced by Wetmore and Merrick ${ }^{5}$. With proteomics not only protein expression is monitored, but also post-translational modifications and protein interactions. These cannot be monitored by transcriptomics and are of substantial value for obtaining full insight in toxicity mechanisms. In addition, several proteins are secreted into body fluids or cell culture medium (secretome). These secreted proteins may play a significant role in the search for toxicity biomarkers, especially in liver research. Because, the liver is responsible for the production and secretion of a large variety of plasma proteins. Furthermore, it is also a major target for drug-induced toxicity, for those reasons changes in secreted protein profiles can reveal relevant toxicity signatures.

Drug-induced hepatotoxicity have several features, for example: steatosis, cholestasis, and necrosis caused by oxidative stress. For a correct classification specific biomarkers need to be determined. Proteomics enables the identification of individual proteins or protein panels reflecting defined hepatotoxic mechanisms.

The development of an in vitro assay based on protein analysis requires the validation of proteome responses of hepatotoxic compounds against in vivo data ${ }^{6}$. 'Omics technologies provide the opportunity to measure similar endpoints of compound-induced changes, enabling in vitro to in vivo comparison.

Ideally, conventional toxicological assays, proteomics and other 'omics-applications are integrated in a systems biology approach. This information will contribute to the development of alternative models for hepatotoxicity screening, characterized by a better prediction of hu- 
man liver toxicity and a reduction of the number of laboratory animals. Furthermore, the application of high throughput screening methods will lead to more cost-effective toxicity testing.

\section{PROTEOMICS TECHNIQUES}

The objective of differential proteomics is to separate, quantify and identify the proteins and peptides in a complex mixture in order to reveal differences of protein expression between experimental conditions. The main current technologies applied for protein separation are either gel-based (1- or 2-dimensional gel electrophoresis (1-DE or 2-DE)), gel-free techniques (Liquid chromatography tandem mass spectrometry (LC-MS/MS)) or a combination of both (Figure 1.1) ${ }^{7}$. Both approaches are complementary since they focus on subsets of proteins that are only partially overlapping. The separation methods are combined with tandem mass spectrometry for the identification of proteins. Here the focus is on 2-DE and LC-MS/MS, since they are currently the most frequently used approaches. The pros and cons of both technologies are summarized in Table 1.1.

\section{Gel-based proteomics}

2-dimensional gel electrophoresis (2-DE)

In 2-DE, the protein sample is first separated by iso-electric focusing on an immobilized $\mathrm{pH}$ gradient gel under the influence of an electric field. The proteins are focused in the gel according to their isoelectric point. Secondly, the proteins are separated according to their molecular mass by sodium dodecyl sulphate polyacrylamide gel electrophoresis (SDS-PAGE). The visualization of proteins after the separation can be achieved by non-specific protein staining either with Coomassie blue, silver, or with fluorescent dyes that are currently the most frequently used ${ }^{8}$. Fluorescent dyes have a high sensitivity and cover a large dynamic range. After staining, the gels are scanned and the protein spots are quantified according their spot intensities. Based on these spot intensities differential protein spots are determined. The main technical drawback of traditional 2-DE is the comparison of various gels corresponding to individual samples. Because gels are treated separately, the risk for technical variation such as alterations in protein migration patterns and staining variability is high. 

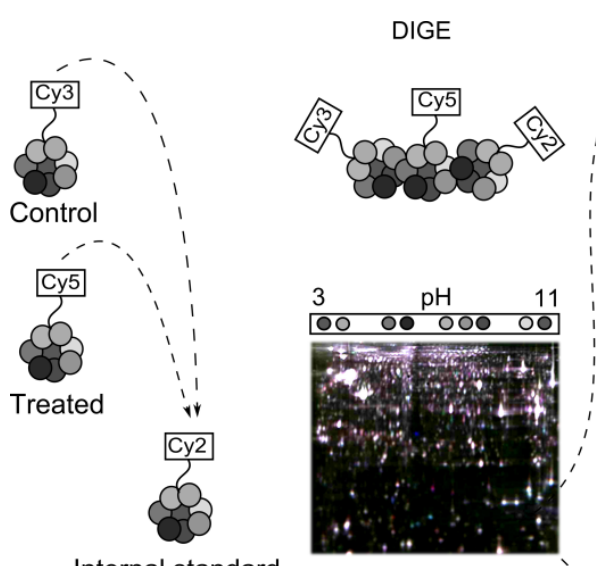

Internal standard
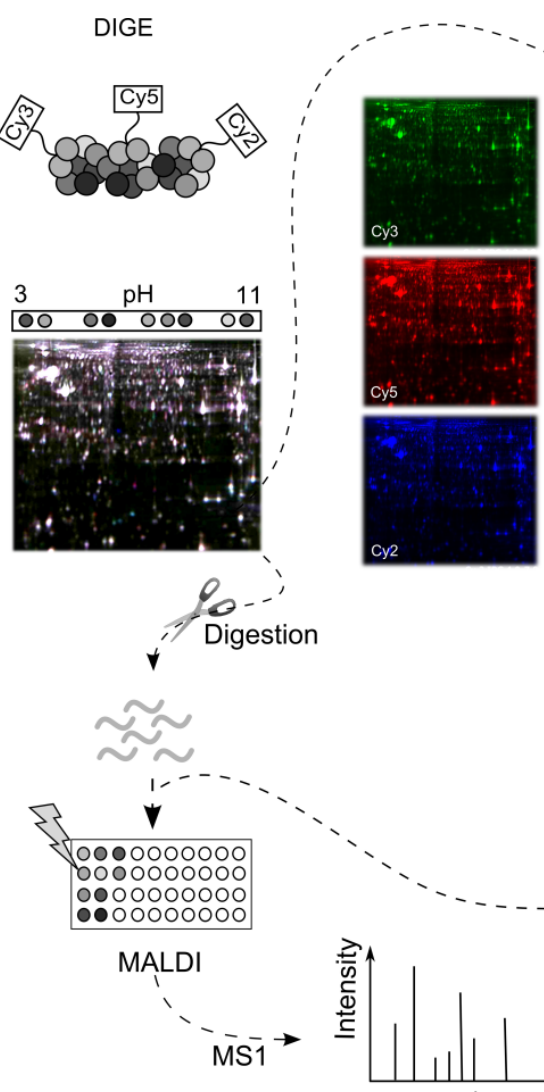

LC MS/MS
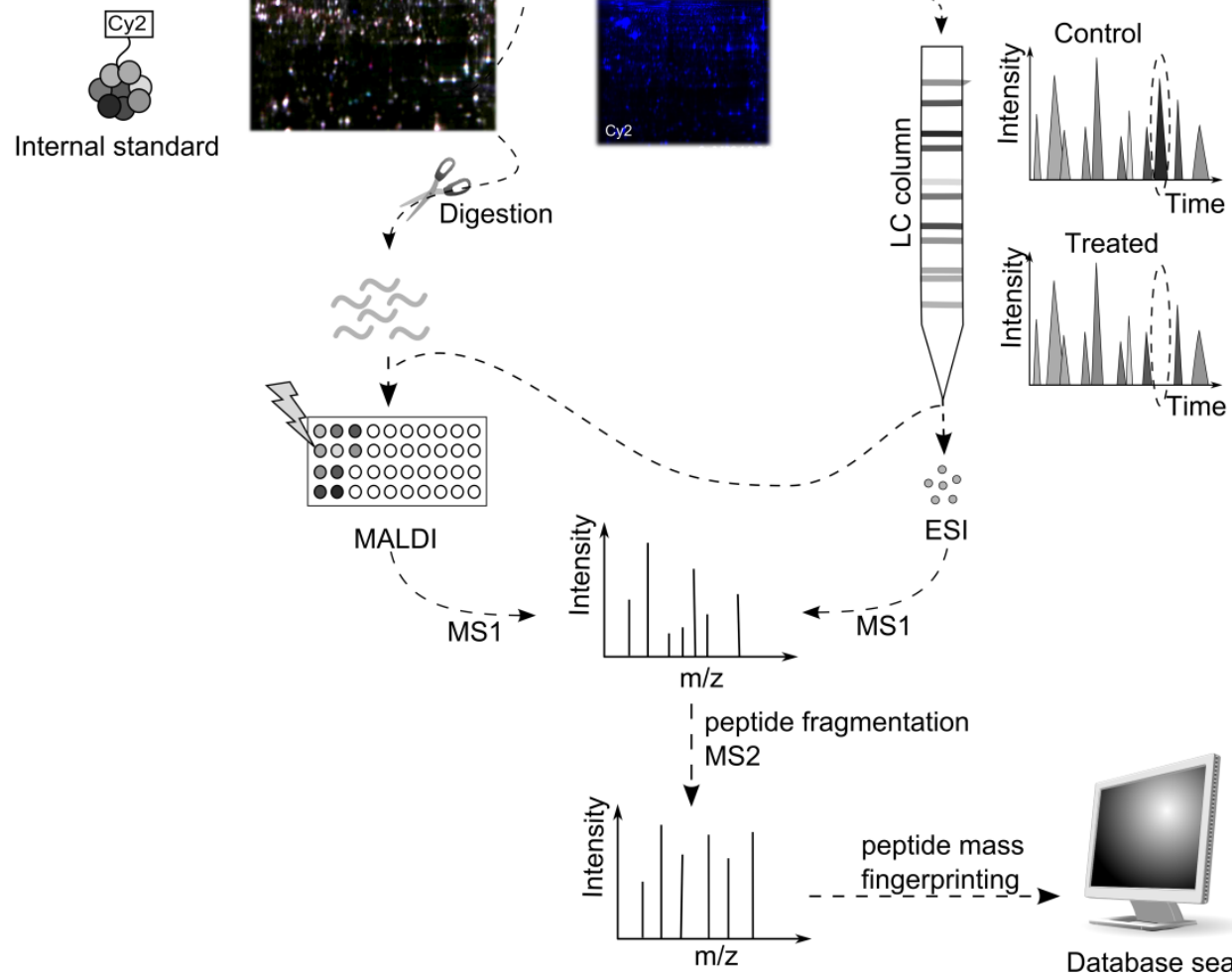

Figure 1.1: overview of the basic principles of DIGE and LC-MS/MS the two mainly used proteomics techniques. On the Left side, the DIGE method combined with mass spectrometry is illustrated. With DIGE the protein samples and internal standard are first labeled with fluorescent dyes (Cy3, Cy5 and Cy2). The internal standard is a an equal mixture of each protein sample. The labeled protein samples are mixed together with the internal standard. Afterwards the protein mixture is separated by isoelectric focussing on an immobilized pH-gradient. After the isoelectric foccusing the proteins are separated according their molecular mass by means of SDS-PAGE. Subsequently, the gel is scanned and analyzed by dedicated software. Afterwards, differentially expressed protein spots are excised and the proteins are digested (usually by trypsin). Afterwards the peptides are introduced into the mass spectrometer by electrospray ionisation (ESI) or spotted on a target plate for matrix assisted laser desorption ionisation (MALDI) mass spectrometry. On the Right side, a LC-MS/MS approach is illustrated. This approach starts with digestion of the protein sample, followed by separation of the peptides by liquid chromatography (LC). In an online LC-MS/MS approach the separated peptides are directly introduced into the mass spectrometer by ESI. In an off-line method the separated peptides are automatically spotted on a MALDI target plate for subsequent MS/MS identification. 
The intrinsic technical variations of classical 2-DE has been strongly reduced by the introduction of difference gel electrophoresis (DIGE). With this technology proteins are labeled with fluorescent dyes prior to separation ${ }^{9}$. Three different fluorescent dyes are available, which allow labeling of two protein samples and one internal standard. The internal standard is a mixture of an equal amount of each protein sample. These labeled protein samples are mixed and separated on one gel according to standard 2-DE method. Subsequently, the gel is scanned and analyzed by dedicated software. The possibility to run two different protein samples on a single gel as well as the introduction of an internal standard on each gel considerably reduces the gel-to-gel variability, thereby improving the classical 2-DE. By this approach the DIGE technology has overcome the limitations of classical 2-DE. For identification of the differentially expressed proteins the protein spots are picked from the gel, digested and introduced into the mass spectrometer by electrospray ionization (ESI) or spotted on a target plate for matrix assisted laser desorption ionization (MALDI) mass spectrometry. Overall, the strength of the 2-DE approach is determined by the accurate and relatively easy quantification of the differentially expressed proteins. Moreover, 2-DE indicates the molecular weight and $\mathrm{pl}$ of proteins. So, different protein iso-forms are easily visualized. Despite the major improvement by the DIGE technology, the 2-DE approach is still hampered by comigration of different proteins on the gel, and has limited applicability for hydrophobic (membrane) proteins and proteins with extreme masses and pl's. Moreover, accurate matching and comparison of the gel images for protein quantification is a time consuming process. This is one of the reasons why shotgun proteomics gained more interest in the last decade.

\section{Blue native gel electrophoresis}

As stated, the 2-DE approach is less suitable for separation of more hydrophobic membrane proteins. To overcome this limitation one can consider blue native polyacrylamide gel electrophoresis (BN-PAGE). In BN-PAGE, the first dimension separation is performed under nondenaturating conditions, which preserve protein-protein interactions and protein complexes, like the oxidative phosphorylation system. Therefore BN-PAGE can provide information about the size, number, stoichiometry, protein composition and relative abundance of protein complexes.

\section{Gel-Free proteomics}

\section{Liquid chromatography tandem mass spectrometry (LC-MS/MS)}

As an alternative for gel-based protein separation, gel-free analysis of complex protein samples by LC-MS/MS has become popular in the last decade, particular the so-called shotgun proteomics approach. With this method protein samples are first digested into complex mixtures of peptides. The peptides are subsequently separated by reversed-phase highperformance LC which is directly coupled to a mass spectrometer by an ESI interface. As an 
alternative the separated peptides can be automatically spotted on a MALDI target plate for MS/MS identification.

Shotgun proteomics enables the detection of more hydrophobic proteins and is considered as high throughput, since thousands of peptides are simultaneously analyzed. However, due to the complexity of the samples, the protein quantification is more challenging. As such, both label-based and label free protein quantification methods have been established for LCMS/MS-mediated proteome analyses.

\section{Isotope-code labeling shotgun technologies}

As gel-free counterpart for DIGE, isotope-coded labeling shotgun technologies were developed. Proteins are labeled with isotopes prior to the separation, e.g. by iTRAQ-Isobaric Tags or by Stable Isotope Labeling with Amino acids in Cell culture (SILAC). Quantification and comparison are performed by detection of the introduced label during MS analysis ${ }^{10}$. Isotope-coded labeling shotgun approaches are considered as accurate technologies for protein quantification. Though, they require expensive isotope labels, specific software, and expertise to analyze data ${ }^{11}$.

\section{Label-free quantification}

Less expensive quantification methods are label free approaches that rely on spectral counting or signal intensity measurement during MS. With spectral counting it is assumed that when more abundant peptides are selected for fragmentation, they will produce a higher abundance of MS/MS spectra, corresponding with the protein amount ${ }^{11}$. However, spectral counting is rather susceptible to variation.

Signal intensity measurement or area under the curve (AUC) relies on the ion abundances at specific retention times for the given ionized peptides, often referred to as ion counts. Partly due to the availability of optimized software, the label-free quantification methods are currently gaining more popularity, compared to the more expensive label-based methods ${ }^{11}$.

\section{Targeted proteomics}

Although both 2-DE and shotgun proteomics are successful techniques to identify candidate biomarkers, validation is required to ensure that those proteins are specific for a certain biological condition. Usually such validations target the protein of interest by applying antibodybased techniques like Western blotting, ELISA or immunohistochemistry. These convenient techniques are widely accepted for the verification and validation of biomarkers. However, when multiple markers or samples need to be analyzed, costs and analysis time will rise. Moreover, suitable antibodies are not available for all potential biomarkers. Therefore, multiple reaction monitoring (MRM) or selective reaction monitoring (SRM) was developed. With this targeted MS approach only the known peptides of interest are selected, while others are ignored which allows the parallel analysis of specific multiple peptide transitions ${ }^{12}$. Therefore 
MRM offers the high throughput and specificity required for the verification of multiple candidate biomarkers. MRM requires a triple quadrupole mass spectrometer, where in the first quadrupole (Q1) the known precursor ion is isolated, in Q2 the ion is fragmented and in Q3 the optimum fragment ions are monitored ${ }^{12}$. Previously proof-of-principle has been delivered by reliable detection of CYP450 enzymes and Udp-glucuronosyltransferases in liver by means of $\mathrm{MRM}^{13}$.

Table 1.1: pros and cons of gel based and gel-free proteomics.

\begin{tabular}{|c|c|c|}
\hline & Gel based proteomics & Gel-free proteomics \\
\hline+ & $\begin{array}{l}\text { - Accurate and relatively easy quantifica- } \\
\text { tion } \\
\text { - Indication of molecular weight and pl so } \\
\text { post-translational modifications are visu- } \\
\text { alized }\end{array}$ & $\begin{array}{ll}- & \text { High throughput } \\
- & \text { Suitable for most proteins }\end{array}$ \\
\hline- & $\begin{array}{l}\text { - Unsuitable for all proteins (depending } \\
\text { their concentration, hydrophobicity, pl } \\
\text { and molecular weight.) } \\
\text { - High abundant proteins can cover low } \\
\text { abundant ones by co-migration } \\
\text { (can be solved by fractionation). } \\
\text { - Matching and comparison of the gel im- } \\
\text { ages is time consuming. }\end{array}$ & $\begin{array}{l}\text { - Quantification is more challenging which } \\
\text { require expensive isotope labels (however, } \\
\text { label-free methods are available), specific } \\
\text { software, and expertise to analyze data. } \\
\text { - } \quad \text { High abundant peptides can cover low } \\
\text { abundant ones by co-elution (which can be } \\
\text { solved by fractionation). }\end{array}$ \\
\hline
\end{tabular}

\section{PROTEOMIC STUDIES ON HEPATOTOXICITY}

\section{In vivo}

In order to be recognized as safe for human consumption or exposure, new compounds need to be tested on rodents or other mammals. In the pharmaceutical industry rats are a preferred model because of their size, ease of manipulation, breeding characteristics, short life span ( \pm 3 years) and because of the in-depth knowledge of the model gained through many years of experience ${ }^{14}$. In vivo studies usually involve 28 or 90 day repeated-dose toxicity tests to evaluate chronic effects, organ toxicity and to establish a non-observable effect level. During these repeated-dose toxicity tests the animals are observed for indications of toxicity. Afterwards, necropsy, blood analysis and histopathology of the organs of the animals are performed.

With in vivo proteomics studies a further insight in the hepatotoxic mechanisms, individual proteins or protein panels reflective for hepatotoxicity can be revealed. Together with other 'omics technologies, proteomics techniques have the potential to detect toxicological effects at earlier time points and lower doses compared as the conventional toxicity assays. 


\section{Tissue proteomics}

Fountoulakis et al. performed proteomics investigation towards acetaminophen toxicity in livers from mice ${ }^{15}$. This study revealed 35 modified proteins after acetaminophen treatment. Some of these proteins were known targets for covalent modification of $\mathrm{N}$-acetyl-pbenzoquinoneimine, which is the most toxic metabolite of acetaminophen ${ }^{15}$.

With the usage of radioactive labeled compounds the toxicant-target proteins can be directly visualized after 2-DE. This technique was used to study bromobenzene-target proteins. For this purpose mice were treated with ${ }^{14} \mathrm{C}$-bromobezene ${ }^{16}$. Afterwards, the liver proteins were isolated and separated with 2-DE, the bromobenzene-target proteins were visualized after measuring the radioactivity of the spots in the gel. In total 33 unique protein targets for bromobenzene were detected including gluthathione S-transferases, protein disulfide isomerases and liver fatty acid-binding protein ${ }^{16}$.

To generate marker panels for carcinogenicity and genotoxicity proteome analysis was used in an in vivo 28-day repeated dose study of 63 chemical compounds ${ }^{17}$. The proteome of rat livers was analyzed by DIGE and the carcinogic characteristic proteins were classified. In this study $79.3 \%$ of the genotoxic compounds and $76.5 \%$ of the non-genotoxic compounds could be correctly classified ${ }^{17}$. In a comparable gene expression signature for predicting nongenotoxic hepatocarcinogens, Fielden et al. ${ }^{18}$ found an accuracy of 63-69\%, whereas the gene expression signature accuracy for predicting non-genotoxic carcinogens was between 55 and $64 \%{ }^{19}$. Although these accuracies still involve a high number of false negative results, proteomics techniques show promising results in the detection of toxicity signatures.

\section{Proteomics of cellular fractionations}

The in vivo proteomics studies described above investigated the overall cellular protein expression. However, when the total proteome of a cell type is examined by 2-DE, low abundant differential proteins can be overshadowed by high abundant proteins. Furthermore, hydrophobic proteins like membrane proteins can be absent from the cell lysate because they are difficult to dissolve. Low abundant or hydrophobic proteins can be enriched by fractionation of the proteome in subcellular fractions like mitochondria, endoplasmic reticulum, microsomes or the cell membrane ${ }^{20}$.

\section{Endoplasmic Reticulum and microsomes}

The endoplasmic reticulum (ER) is a key organelle for protein secretion and is involved in the synthesis of both proteins and lipids ${ }^{21}$. Moreover, the ER is involved in the detoxification of xenobiotic compounds, which explains its attractiveness for toxicological studies ${ }^{21}$. To acquire in-depth knowledge of the cellular and ER protein expression, Zgoda et al. ${ }^{22}$ has fractionated the proteome of liver samples into a cytosolic and a microsome fraction. Microsomes are small vesicles derived from the ER when the cells are mechanically disrupted and contain high amounts of cytochrome P450 metabolizing enzymes ${ }^{23}$. The proteome of liver samples derived from mice treated with phenobarbital and 3-methylcholanthrene were fractionated in a 
cytosolic and a microsome fraction. Both fractions were subjected to 2-DE ${ }^{22}$. Despite the fractionation, the cytosolic fraction revealed more information than the subcellular microsome fraction. Microsomal changes induced by phenobarbital and 3-methylcholanthrene were quite similar. While the cytosolic response induced by phenobarbital showed distinct differences from the 3-methylcholanthrene-induced response ${ }^{22}$. A high amount of CYP450 enzymes was expected in the microsomal fraction, however only two CYP450 enzymes were detected. This can be explained by the fact that CYP450 enzymes are membrane-associated proteins which are difficult to analyze by means of 2-DE. Therefore, shotgun proteomics is probably more suitable for analyzing the differential expression of cytochrome P450 metabolizing enzymes ${ }^{24,25}$. For example, microsomes isolated from carbon tetrachloride $\left(\mathrm{CCl}_{4}\right)^{-}$ treated rat liver were used to analyze the changes in protein expression of the CYP450 enzymes $^{26}$. From a 1-DE gel the area from 45-66 kDa was excised, since this corresponds with the molecular weight of most CYP450 proteins. Subsequently an acetylation stable isotopic labeling method coupled with Linear Trap Quadrupole Fourier Transform Ion Cyclotron Resonance mass spectrometry (LTQ-FTICR) was applied. With this approach the researchers were able to identify and quantify 17 cytochrome P450 proteins. Among them, the expression of $2 \mathrm{C} 11,3 \mathrm{~A} 2$, and $2 \mathrm{E} 1$ was down-regulated, while that the expression of $2 \mathrm{C} 6,2 \mathrm{~B} 2$, and $2 \mathrm{~B} 1$ was up-regulated $^{26}$.

\section{Mitochondria}

Mitochondria are responsible for the oxidation of fatty acids into acetyl-CoA through the $\beta$ oxidation. Impairment of this $\beta$-oxidation process, either drug-induced or through long-term imbalance between food intake and energy expenditure, will lead to fat accumulation in hepatocytes and ultimately steatosis. Therefore, analyzing mitochondrial proteins can reveal mechanistic details in the development of drug-induced steatosis, although mitochondrial dysfunction is not exclusive for steatosis. Bailey et al. described 2-DE and BN-PAGE to analyze mitochondrial proteins in alcoholic liver disease ${ }^{27}$. Both gel-based techniques were used to study the mitochondrial liver proteome of rats with ethanol-induced hepatotoxicity ${ }^{28}$. In total 43 differentially expressed proteins were identified, comprising enzymes of the $\beta$-oxidation cycle, nuclear encoded subunits of the oxidative phosphorylation system, mitochondrial chaperones and enzymes of amino acid metabolism. Furthermore a decrease of several polypeptides of the respiratory complexes in the ethanol treated rats was observed.

\section{Plasma/serum proteome}

Since most proteins in blood are secreted by the liver, plasma or serum is a potential source for hepatotoxicity biomarkers, represented by aberrantly secreted proteins or proteins leaked from the liver due to injury. This is demonstrated by the current liver function tests where the enzymatic activity of alanine aminotransferase, aspartate aminotransferase, gamma glutamic transpeptidase, lactic dehydrogenase and alkaline phosphatase are measured in blood. In addition, blood is easily accessible and practical for repeated sampling and analysis. Previously, the protein expression in Z24-treated rat livers was studied in parallel with the plasma proteome from the animals ${ }^{29}$. $\mathrm{Z} 24$ is a synthetic anti-angiogenic compound that inhibits 
growth and metastasis of tumors. However, it was shown that Z24 induces hepatotoxicity in rodents. The rats were administered once daily with Z24 for 5 days at doses of 0,50 , 100, or $200 \mathrm{mg} / \mathrm{kg}$ per day. Twenty-four hours after the final administration, blood samples and whole livers were collected. From these samples differentially expressed proteins were analyzed with 2-DE and MALDI-TOF/TOF MS, 22 non-redundant liver proteins and 11 plasma proteins were found differentially expressed. These proteins are involved in several important metabolic pathways, including carbohydrate, lipid, amino acid, and energy metabolism, biotransformation, and apoptosis. Most of the identified hepatic proteins were located in mitochondria, where Z24 also increased the ROS production and decreased the NAD(P)H levels. Therefore, it was concluded that Z24 inhibits the aerobic carbohydrate oxidation, fatty acid $\beta$ oxidation, and oxidative phosphorylation pathways resulting in mitochondrial dysfunction and apoptosis-mediated cell death. In addition, potential biomarkers for Z24-induced hepatotoxicity, namely fetub protein and argininosuccinate synthase were detected in the plasma ${ }^{29}$. These two proteins were also found differentially expressed together with several other proteins in the serum of rats 24 hour after acetaminophen exposure ${ }^{30}$.

\section{In vitro}

The current in vivo repeated-dose toxicity studies, which include hepatotoxicity screening, have often only a limited reliability and involve numerous animals. Therefore, properties of possible non-animal-based models and their ability to classify toxic compounds need to be evaluated as potential replacements. The most commonly used in vitro models in hepatotoxicity studies are cell lines like HepG2, HepaRG and primary hepatocytes or liver slices from several species. The most frequently used species are rat, mouse and human ${ }^{6,31-35}$. In addition, recent developments in stem cell-derived hepatocytes have led to their use in toxicological investigations ${ }^{36}$.

The baseline expression of the biotransformation genes in several cell models (primary human hepatocytes, HepG2, HepaRG and stem-cell-derived hepatocytes) were compared with the expression human liver and their biotransformation pathways were statistically ranked ${ }^{37}$. The expression pattern of the biotransformation genes of both primary hepatocytes and HepaRG cells was similar to those of human liver samples. Whereas the expression pattern of HepG2 did not. Two analyzed stem-cell-derived hepatocytes had only a few biotransformation genes with an expression comparable with those from the normal liver samples and primary hepatocytes ${ }^{37}$. According to the ranking test of the meta pathway for biotransformation, the liver cell models can be placed in following order: human liver, primary human hepatocytes, HepaRG > HepG2 > human stem cell models ${ }^{37}$. This study can be complemented with results from the baseline protein expression of the cell models. Below, the basal protein expression of the most commonly used in vitro models is discussed, including the results of hepatotoxic proteome studies. 


\section{Precision cut liver slices}

Hepatotoxicity is often the result of multi-cellular processes in the liver. In precision cut liver slices the three-dimensional structure of the liver is preserved and contains all the cell types present in the liver in vivo. The presence of other cells than hepatocytes, like Stellate and Kupffer cells, adds a significant value to this model as these cells often play an important role in the mediation of hepatotoxicity ${ }^{38}$. On the other hand, the presence of different cell types will increase the variation in the study; consequently the slices must be isolated in a controlled manner. For their use in toxicological research it is essential that the liver slices maintain their metabolic activity to ensure the biotransformation of xenobiotic compounds. Unfortunately, the use of precision cut liver slices is hampered due to their short life span, the poor penetration of compounds to the inner cell layers, the limited availability of donors for human liver slices and the great inter-individual variation, of which the latter can be minimized by using sufficient biological replicas. So far, studies on the toxicological proteome of liver slices have not been published, probably due to the fact that liver slices are not commonly used. Therefore, there is still limited knowledge about the intercellular interactions in liver toxicity mechanisms. Hence, if their life span, which is now around 16 hours, could be increased, the liver slice model would become increasingly important for hepatotoxicity testing in the future.

\section{Primary hepatocytes}

Primary hepatocytes are considered as 'the gold standard' for in vitro xenobiotic metabolism and toxicity studies. They express both phase I and phase II enzymes, which are necessary for biotransformation of xenobiotic compounds, and maintain other liver specific functions. Considering these characteristics, primary hepatocytes will probably predict toxicity for xenobiotic compounds in a better way as compared to cell lines ${ }^{39}$. Seglen ${ }^{40}$ introduced the two-step collagenase perfusion to obtain primary hepatocytes from rats, a technique that is now increasingly used. The primary hepatocytes need to be cultured in a sandwich configuration of extracellular matrix proteins, like collagen I. This sandwich configuration mimics the three dimensional structure of the liver that retains the original functions of the hepatocytes and increases their life span ${ }^{41}$. After isolation, a recuperation period of 42 hours needs to be taken into account before the hepatocytes can be exposed to any toxicological compound. In this recuperation period, gap junctions as well as bile canalicular networks will be formed between the hepatocytes ${ }^{31}$, as can be seen in Figure 1.2. 


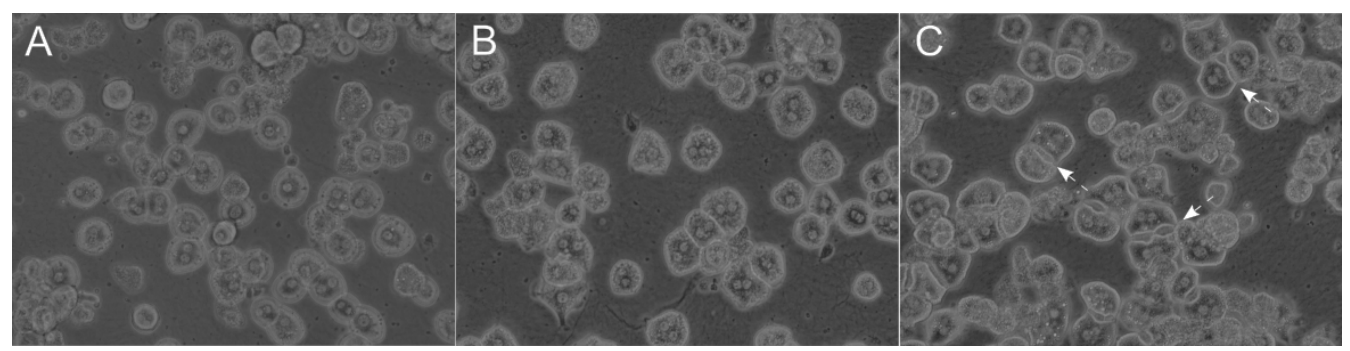

Figure 1.2 Primary mouse hepatocytes (A) after isolation, (B) after a recuperation period of 16 hour and (C) after a recuperation period of 42 hour. After 42 hours the bile canalicular networks (indicated by arrows) will be formed

\section{Rodent hepatocytes}

The rat is the most frequently used rodent species for the preparation of primary hepatocytes. Until now most in vivo toxicity studies are performed in rats, therefore the protein expression measured in vivo can directly be linked to proteome data from primary hepatocytes. For the prediction of drug-induced hepatotoxicity several studies have been conducted to investigate differential protein expression after administration of hepatotoxic compounds (acetaminophen, amiodarone, tetracycline and carbon tetrachloride) to rats in vivo or to isolated primary rat hepatocytes ${ }^{42-45}$. These comparable in vitro and in vivo experiments enable the in vitro to in vivo comparisons of the protein expression profiles induced by well-known hepatotoxicants. Primary rat hepatocytes exposed to the hepatotoxicants, revealed the differentially expression of proteins related to oxidative stress and mitochondrial metabolism regulation ${ }^{43,44}$. The results of these in vitro experiments appeared to be well in line with the protein expression of similar in vivo studies ${ }^{42,45}$. Oxidative stress with an altered mitochondrial metabolism often indicate the development of necrosis.

A compound in preclinical development, referred as CDA, was found to induce hepatocellular steatosis in vivo. In a first experiment using DIGE analysis, it was shown that $250 \mathrm{mg} / \mathrm{kg} \mathrm{CDA}$ induced hepatotoxicity in rats 6 hours after treatment ${ }^{46}$. Several identified differentially expressed proteins could be associated with known toxicological mechanisms involved in liver steatosis. These protein changes occurred before the onset of clinical biochemistry parameters, suggesting that these proteins could represent potential early markers of CDA-induced hepatotoxicity ${ }^{46}$. This study was extended by an in vitro experiment in primary hepatocytes, to investigated whether this information was also represented in vitro ${ }^{47}$. Pathway analysis showed that CDA predominantly affected cell death and cellular assembly and organization. This included alterations in secreted proteins, endoplasmic reticulum and mitochondrial chaperones, antioxidant proteins, and enzymes involved in fatty acid biosynthesis.

In vitro to in vivo comparisons showed that hepatotoxic responses of primary rat hepatocytes at protein level are well preserved compared to the hepatotoxic responses in vivo ${ }^{42-47}$.

The isolation of primary hepatocytes may entail a decreased expression of the detoxifying capacities. This may result in a decreased hepatotoxic response after exposure to indirect 
acting toxicants, which require the activation by drug metabolizing enzymes. The hepatotoxic responses of direct and indirect acting toxicants (aflatoxin B1, cadmium, N-methyl- $\mathrm{N}^{\prime}$-nitro-Nnitrosoguanidine, methyl methanesulfonate, carbon tetrachloride, N,N-dimethylformamide, vinyl acetate and acetaminophen) in primary rat hepatocytes was investigated by Farkas and Tannenbaum ${ }^{48}$. Aflatoxin $B_{1}$ and the direct acting toxicants induced a significantly decrease of the urea and albumin secretion. Where this was not the case for the indirect toxicants. Western blotting showed that the primary rat hepatocytes maintained the protein expression of the cytochrome P450 enzymes CYP1A, 2B, 3A2 but gradually have lost the expression of CYP2E1. CYP2E1 is one of the main enzymes responsible for the metabolic activation of acetaminophen ${ }^{49}$, carbon tetrachloride ${ }^{50}$, and $\mathrm{N}, \mathrm{N}$-dimethylformamide ${ }^{51}$. The partial loss of CYP2E1 can explain the resistance of the hepatocytes to these indirect compounds.

The primary rat hepatocytes and to a lesser extent primary human hepatocytes, are wellestablished in vitro systems. Primary mouse hepatocytes however, are less commonly used in toxicity studies although transgenic mouse models provide an opportunity to study specific toxicity mechanisms. Moreover, compared to primary rat hepatocytes, primary mouse hepatocytes are more stable and remain their metabolic competence for a longer period ${ }^{31}$. Primary mouse hepatocytes from different strains showed only little differences between cellular function and expression of liver specific enzymes. Furthermore, the cytotoxicity of three model compounds acetaminophen, WY-14,643 and rifampin did not differ much between the strains ${ }^{52}$. Therefore, primary mouse hepatocytes have not only potential for toxicity screening, they can also serve as an alternative for population diversity ${ }^{52}$.

\section{Primary human hepatocytes}

Similar to human liver slices, studies with primary human hepatocytes are limited by the scarcity of suitable liver samples. Furthermore, they are hindered by a great inter-individual variation ${ }^{53}$. The isolation of human hepatocytes is also based on the two-step collagenase perfusion of Seglen ${ }^{40}$ but adapted to the ex vivo treatment of human liver from a donor ${ }^{54}$. Until now the proteome of primary human hepatocytes has not frequently been evaluated in hepatotoxicity studies. One study showed the effects of bezafibrate on the proteome of primary human hepatocytes ${ }^{55}$. In contrast to primary rat hepatocytes, the expression of CYP4A was not induced in primary human hepatocytes. This study indicates the differences of the drugmetabolizing enzymes between species, and emphasizes the use of primary human hepatocytes to overcome issues related to interspecies variation ${ }^{55}$.

\section{Cell lines}

Liver cell lines are commonly used in hepatocellular and toxicity studies ${ }^{6,56-58}$. These models are a relatively simple system, since they are easy to maintain and cultivate. Several studies compared hepatic cell lines with primary hepatocytes. Most of these studies are based on the comparison of gene expression profiles at the transcriptome level which poorly reflect the differences at the proteome level ${ }^{59}$. 
Slany et al. ${ }^{6}$ applied 2-DE analysis and shotgun proteomics to compare cellular and secreted proteins from the hepatoma cells HepG2 and Hep3B with primary human hepatocytes. Both 2-DE and shotgun proteomics of the cellular proteome lead to the conclusion that HepG2 cells express more liver specific proteins than Hep3B, while Hep3B displayed more commonalities to skin fibroblasts. The cellular proteome revealed 104 liver specific proteins in primary human hepatocytes, 20 in HepG2, and only 6 in Hep3B. With shotgun proteomics, 32 detoxifying proteins were found in primary human hepatocytes and only 4 in HepG2 and 2 in Hep3B. This illustrates the loss of the detoxifying capacities in hepatic cells lines compared to primary hepatocytes. With respect to secreted proteins, 46 out of 72 proteins identified in the secretome of primary hepatocytes were plasma proteins characteristic for hepatocytes. In HepG2 this was 55 out of 139, while in Hep3B this was only 24 out of $72^{6}$. Analyses of the secretome showed that HepG2 cells secrete a considerable amount of plasma proteins such as albumin, serotransferrin, apolipoproteins and fibrinogen, which is a characteristic function of hepatocytes ${ }^{6}$. Hep3B cells however secreted only a relatively small amount of plasma proteins, while other secreted proteins were not characteristic for liver cells ${ }^{6}$.

The suitability of HepG2 as an in vitro cell system for toxicity screening was investigated by comparing the differential protein expression of HepG2 cells with the in vivo protein expression in rats after administration of 6 hepatotoxicants (1-naphthyl-isothiocyanate, indomethacine, acetaminophen, cisplatin, tetracycline, dimethyl-nitrosamine) and 4 non-toxic compounds (aspirin, isoproterenol, phenylephrine and DMSO) ${ }^{56}$. This comparative proteome analysis demonstrated the functional differences between HepG2 cells and the in vivo situation. The in vivo rat system rather reflected the full, although species-specifically biased, dynamics of a liver which could not be completely replaced by HepG2 cells. Nevertheless, 8 of 13 potential toxicity marker proteins found in rat liver were also detected in HepG2 ${ }^{56}$. In addition, HepG2 cells showed the quality of an in vitro testing system for detecting hepatotoxicity in an early stage of drug discovery ${ }^{56}$.

The proteome of the mouse hepatoma cell line Hepa1- 6 was compared with primary mouse hepatocytes ${ }^{59}$. Bioinformatic analysis of both proteomes showed a deficient mitochondrial activity in Hepa1-6 cells, reflecting re-arrangement of metabolic pathways, drastically upregulated cell cycle-associated functions, and largely inactive drug metabolizing enzymes ${ }^{59}$. From the comparative studies between primary hepatocytes and hepatic cell lines it can be concluded that hepatoma cell lines still have liver-specific functions and can be a valuable tool in toxicity screening. Nevertheless researchers should be aware of the fading of several specific functions, such as activity of cytochrome P450 enzymes due to the immortalization of the cells. This was demonstrated in the hierarchical clustering analysis of the expressed proteins of nine hepatocellular carcinoma cell lines and primary human hepatocytes from five individuals, where all cell lines were distinguished from the primary hepatocytes ${ }^{60}$. Therefore these cell lines will not always be successful in hepatotoxicity screening, particularly with respect to xenobiotics which need biotransformation/activation before they become toxic. 


\section{In vitro secretome}

The secretome of cells and tissues is a rich source of potential markers, since it reflects a broad variety of pathological conditions. Moreover, secreted biomarkers are readily accessible and can therefore easily be measured in the cell culture medium. One of the major functions of the liver is the production of serum proteins. Consequently, when specific protein markers for hepatotoxicity can be found in the secretome of hepatocytes, it is likely that these markers are present in serum and available for diagnosing hepatotoxicity in patients or subjects from clinical trials. However, the analysis of proteins secreted by cultured cells is often a challenge because they can be masked by non-secreted proteins. Cultivation of cells is unavoidably accompanied by cell death. Consequently, significant amounts of cytoplasmic proteins may be released into the medium. By selective inhibition of secretion pathways it is possible to discriminate secreted from non-secreted proteins. The inhibition of these pathways can be obtained for example with Brefeldin A that is known to inhibit protein secretion by interfering with the function of the Golgi apparatus. Alternatively, incubation at $20^{\circ} \mathrm{C}$ inhibits both the ER/Golgi-dependent and independent pathways ${ }^{61}$. The differentially expressed proteins between the non-inhibited and the inhibited conditions are considered as genuinely secreted. Ideally this is verified with bioinformatics tools. To improve the analysis of secreted proteins, Zwickl et al ${ }^{35}$ have used metabolic labeling of proteins that are synthesized during a limited incubation period of HepG2 and human liver slices and combined it with 2-DE. Whereas fluorescent staining of the gel led to the detection of a large number of proteins derived from residual plasma and dead cells, the autoradiographs selectively displayed genuinely secreted proteins. Therefore this technique can improve the specific detection of secreted proteins $^{35}$.

Previously, the proteins secreted by HepG2/C3A cells in response to ethanol exposure were investigated ${ }^{62}$. All differentially expressed proteins from this study are related with known in vivo effects of ethanol exposure. These effects vary from apoptosis and inflammation to cell leakage from disturbed cells, indicating that this model can be used for the identification of potential toxicity markers ${ }^{62}$. In addition, the value of the secretome of HepG2 is confirmed by a study of Choi et al. ${ }^{57}$ where potential biomarkers for the genotoxic compound di(2ethylhexyl)phthalate were found in the secretome of exposed HepG2 cells ${ }^{57}$. In this study the proteins were analyzed by 2-DE using two different pl ranges (4-7 and 6-9) combined to a large size 2-DE gel. This revealed 35 differential proteins belonging to several functional groups ${ }^{57}$. Based on the differentially expressed proteins, di(2-ethylhexyl)phthalate was found to affect the formation of cell structure, apoptosis, and tumor progression.

Analysis of proteins secreted by primary rat hepatocytes exposed to aflatoxin B1 showed a decreased expression of $\alpha 2$-macroglobulin and $\alpha 1$-antitrypsin ${ }^{34}$. Patients with $\alpha 1$-antitrypsin deficiency have an increased risk for liver carcinoma and cirrhosis; these symptoms are also seen with aflatoxin B1 intoxication. Therefore, it is likely that the decreased expression of these protease inhibitors in the medium is linked to an impairment of the liver. 
In another study the medium of human immortalized hepatocytes with an over-expression of the CYP3A4, the most common type of CYP450 enzyme in human liver, was studied after exposure to hepatotoxic (troglitazone, ciglitazone, farglitazar, ritonavir) and non-hepatotoxic compounds (DMSO, indinavir, rosiglitazone, tesaglitazar) ${ }^{63}$. The cells were incubated with the compounds for 20 hours and afterwards the conditioned medium was analyzed with LCMS/MS. This analysis revealed two proteins, referred to as BMS-PTX-265 and BMS-PTX-837, that were significantly increased in the secretome of the cells treated with each of the hepatotoxic compounds. The response of these two proteins to an expanded set of 20 compounds was further analyzed. For all 20 drugs, elevations of BMS-PTX-265 correlated exactly with the known safety profile; whereas changes in BMS-PTX-837 correctly predicted the safety profile from 19 of the 20 drugs (one false negative) ${ }^{63}$. This study exemplifies that proteomics and particularly secretome analysis can reveal biomarkers for hepatotoxicity screening.

\section{PROTEOMICS IN RELATION TO OTHER 'OMICS TECHNIQUES}

Toxicological effects is often a result of complex interactions at multiple levels within biological systems. Therefore, to obtain a complete picture of the toxicological processes transcriptomics, proteomics and metabolomics should be studied in parallel.

Bromobenzene-induced hepatotoxicity was analyzed in a combined transcriptomics and proteomics experiment ${ }^{64}$. By using principle component analyses (PCA) the treated samples were clearly distinguished from control samples, as well on the transcriptome as on the proteome level. However, direct comparison of the transcriptomics and proteomics data showed only a modest overlap. In addition, parallel analysis of gene and protein expression in rat liver after acetaminophen administration, did not detect a simultaneous expression of the mRNA and their corresponding proteins ${ }^{65}$. The overlap between gene and protein expression is often limited because, the coverage of the proteins detected by proteomics is usually much lower the thousands of genes analyzed by means of microarray analysis. Furthermore, some proteins are stored in the cytoplasma and released upon the insult before their transcription is initiated to refill the cytoplasmic store. Another explanation is that differentially expressed proteins detected with proteomics are often differential due to posttranslational modifications, which do not appear at the transcriptome level. However, despite the wide range of the 'omics-data and the different ways of interpreting these results, the overall outcome is usually independent of the tools applied ${ }^{2,65}$.

Several public or private partnerships have made an effort to integrate 'omics data and techniques for a better mechanistic understanding of toxicity. The Chemical Effects in Biological Systems (CEBS) is an integrated public repository for toxicogenomics data, which allows the user to integrate various data types and allows studies on the early prediction of key toxicities. It is available at http://cebs.niehs.nih.gov and described by ${ }^{66}$. This database includes 27 toxicogenomics studies from which 22 are of rat, 4 are of mice and 1 is of Caenorhabditis 
elegans ${ }^{66}$. However, 26 studies are based on transcriptomics data and only one contains proteomics data.

In the EU Framework 6 project: Predictive Toxicology (PredTox), the effects of 16 different compounds were analyzed by several 'omics techniques together with conventional toxicological parameters ${ }^{2}$. The compounds used in this project included 14 pharmaceuticals which were discontinued at certain stages of preclinical development due to toxicological findings in liver and/or kidney after 2- to 4-weeks in systemic rat studies. Beside those 14 proprietary drug candidates, 2 reference toxic compounds (gentamicin and troglitazone) were used ${ }^{2}$. These studies were focused on hepato- and nephrotoxicity caused by the selected compounds. From this study liver, kidney, blood and urine samples were collected for transcriptomics, proteomics and metabolomics. Again the comparison of both techniques was hindered by the lower coverage of the detected proteins compared to detection of thousands of genes analyzed by micro-arrays. However, toxicological mechanisms identified by proteome were similar to the results from the transcriptome analysis ${ }^{67}$. For NMR analysis urine appeared to be the most informative bio fluid, which could discriminate between metabolites that were confirmed by histopathology. From all 'omics approaches, target organ transcriptomics revealed the most information for the generation of mechanistic models ${ }^{2,68}$.

The Liver Toxicity Biomarker Study (LTBS), analyzes the biochemical effects of drugs which can cause hepatotoxicity ${ }^{69}$. In this project will study 5 compound pairs, with each a similar therapeutic mechanism, in a 28-day rat study. Liver and body fluids will be collected to perform transcriptomics, proteomics and metabolomics. The preliminary results from the first phase of the project revealed 2 compounds (entacapone and tolcapone) with promising marker analytes ${ }^{69}$. The LTBS study illustrates the value of the broad-spectrum, molecular systems analysis to study hepatotoxic effects.

\section{STUDY HYPOTHESIS OF THIS THESIS}

The liver is responsible for the metabolism and elimination of chemical compounds and consequently, it is the main target for drug-induced toxicity. Therefore, the identification of potential hepatotoxic compounds represents a critical step in the development of new drugs. More sensitive and reliable endpoints are necessary to detect hepatotoxicity at an early stage of drug discovery. With the introduction of toxicogenomics, the number of simultaneously measurable endpoints increased remarkably. In addition, the toxicological endpoints measured in vivo by means of toxicogenomics, are similar to the endpoints measured in vitro. This indicates the value of toxicogenomics in the development of in vitro screening assays predictive for hepatotoxicity in humans. Besides the reduction of used laboratory animals, these in vitro models can contribute to the identification of potential hepatotoxicity at an earlier stage of drug discovery. Especially hepatocyte-based in vitro models like hepatic cell lines, precision-cut livers slices and primary hepatocytes are of interest. The use of primary hepatocytes is recommended because of their metabolic capacities. Primary mouse hepatocytes in par- 
ticular, have shown to be a promising in vitro model for screening purposes. They are metabolic competent and were able to discriminate genotoxic compounds from non-genotoxic compounds ${ }^{31,70}$. Moreover, transgenic mouse models are available, which may provide primary hepatocytes suitable for dedicated mechanistic investigations of liver toxicity.

Currently, several approaches to study gene expression have been conducted to search for appropriate endpoints to improve the current toxicity tests ${ }^{33,71-74}$. However, the mRNA expression is not always predictive for the expression at protein level demonstrating the complexity in regulation of gene expression ${ }^{75}$. Nevertheless, reports on the protein expression related to hepatotoxicity are still limited. This indicates that there is need of proteome analysis in the field of toxicogenomics.

This PhD project is based on the hypothesis that a toxicoproteomics approach enables the detection of specific fingerprints in protein expression induced by well-defined hepatotoxicants. This would allow the classification and identification of hepatotoxic compounds in in vitro hepatic cell systems and short term in vivo models.

To obtain specific protein fingerprints to classify hepatotoxic compounds according their hepatotoxic phenotype, it is essential to use well-defined hepatotoxicants. Based on their in vivo phenotype, most hepatotoxic compounds can be classified as inducers of necrosis, steatosis or cholestasis.

Oxidative stressors induce excessive injury to mitochondria and other cell organelles through free radicals and/or toxic metabolites. This excessive injury can lead to the development of necrosis, a form of premature cell death.

Compounds which cause steatosis are thought to induce mitochondrial damage through impairment of the fatty acid $\beta$-oxidation and accumulation of small lipid vesicles within hepatocytes. A severe decrease in energy production may cause hepatic failure, coma and death.

Cholestasis represents failure of bile excretion. It is often caused by compounds which inhibit bile acid transport proteins. That leads to accumulation of hepatocellular bile acids resulting in liver damage.

The compounds used in the studies described in this thesis have been selected to be indicative for necrosis, steatosis or cholestasis based on their in vivo phenotype as determined from extended literature search. Prototypical compounds chosen for these three categories are amiodarone for steatosis, cyclosporin A for cholestasis and acetaminophen for cytotoxicity by oxidative stress. In a final study this list of hepatotoxic compound is extended with tetracycline and valproic acid (steatosis), chlorpramazine and ethenylestradiol (cholestasis), diclofenac and paraquat (necrosis)and two nephrotoxicants: D-mannitol, $\mathrm{LiCO}_{3}$.

By means of proteomics expression profiles indicative for the different phenotypes of hepatotoxicity (necrosis, cholestasis and steatosis) have been generated. Besides screening of the cellular proteome, proteomics techniques enable the analysis of secreted proteins. Previously it has been shown that liver injury affects lipid metabolism and protein secretory activity as 
exemplified by altered secretion of lipoproteins and coagulation factors. Therefore, it was decided to analyze the secretome additionally to the analysis of the cellular proteome.

This PhD project is focused on a proteomics approach. However, biological phenomena cannot be fully explained by a single 'omics platform. Instead the net interactions of global cellular and biochemistry components within a cell or organism are required. Therefore proteome analysis data was partially supplemented with complementary transcriptome data. However a direct comparison of proteomic and transcriptomic expression data from divergent platforms remains challenging, because the coverage of differentially expressed proteins detected with proteomics technologies is still less than the gene alterations covered by microarray procedures.

\section{OUTLINE OF THIS THESIS}

The human hepatoma cell line HepG2 is a well-known in vitro model which is frequently used in toxicity studies. In chapter $\mathbf{2}$ we investigated its value for screening purposes of hepatotoxic compounds. The changes in protein expression of HepG2 after exposure to acetaminophen, amiodarone and cyclosporin A, were studied using DIGE.

The use of primary human hepatocytes is preferred in toxicological studies. However, their use is hindered by limited availability and inter-individual variation. These barriers may be overcome by using primary mouse hepatocytes. In chapter $\mathbf{3}$ we analyzed the cellular proteome of primary mouse hepatocytes for its ability to detect drug-induced hepatotoxicity, and the level of conservation of responses in comparison with a human in vitro model. For that reason we analyzed the protein expression in primary mouse hepatocytes after exposure to acetaminophen, amiodarone and cyclosporin $\mathrm{A}$ and compared it with the protein expression in HepG2, as described in chapter 2.

The cellular proteome of primary mouse hepatocytes revealed that cyclosporin A induce endoplasmic reticulum (ER) stress and altered the ER-Golgi transport probably resulting in an inhibited protein secretion. This prompted us to analyze the secretome of these primary mouse hepatocytes after administration of the same hepatotoxic compounds, which is described in chapter 4.

Analysis of one 'omics platform can only partially explain the occurrence of biological events. Previous chapters describe studies performed on HepG2 and primary mouse hepatocytes evaluated by a limited set of proteins by means of DIGE. With use of DNA-microrrays it is possible to study simultaneously the expression of thousands of genes. In chapter $\mathbf{5}$, we applied a global toxicogenomics approach to compare the whole-genome gene expression between HepG2 and primary mouse hepatocytes after the exposure to acetaminophen, cyclosporin A and amiodarone. 
In order to reduce the amount of laboratory animals, alternative in vitro models (HepG2 and primary mouse hepatocytes) are evaluated for their screening properties. Ideally these in vitro models reflect the in vivo toxicological response. Accordingly, in vitro to in vivo comparison is necessary. In chapter 6 the hepatic protein expression from C57BL/6 mice after CsAinduced cholestasis was examined. The development of cholestasis at the proteome level was analyzed after 4, 11 and 25 days of daily doses of CsA. The cholestatic phenotype was established after 25 days and was confirmed by serum biochemistry and histopathology. To establish an in vitro-in vivo comparison of CsA-induced protein expression profiles the results from our previous studies with HepG2, described in chapter 2 and primary mouse hepatocytes, described in chapter 3 , were used.

Previously, acetaminophen, amiodarone and cyclosporin A were used as model compounds for necrosis, steatosis and cholestasis, respectively. Chapter 7 describes a study in which compounds inducing similar toxicological endpoints were examined for comparable changes in protein expression. Primary mouse hepatocytes were exposed to six different wellcharacterized hepatotoxicants: diclofenac and paraquat (necrosis), tetracycline and valproic acid (steatosis), chlorpramazine and ethenylestradiol (cholestasis), and two nephrotoxicants: D-mannitol, $\mathrm{LiCO}_{3}$. Afterwards changes in protein expression were determined. By using a discriminant principal component analysis we attempted to differentiate between the hepatotoxic phenotypes. For this analysis we have included the protein spot maps from our previous study where primary mouse hepatocytes were exposed to acetaminophen, amiodarone and cyclosporin A, inducing necrosis, steatosis and cholestasis, respectively.

Finally, chapter $\mathbf{8}$ presents the summary and discussion of the major findings of all these studies and a general discussion to place the results in the context of hepatotoxicity.

\section{REFERENCES}

1 Olson, H., Betton, G., Stritar, J. \& Robinson, D. The predictivity of the toxicity of pharmaceuticals in humans from animal data--an interim assessment. Toxicol Lett 102-103, 535-538 (1998).

2 Suter, L. et al. EU Framework 6 Project: Predictive Toxicology (PredTox)-overview and outcome. Toxicol Appl Pharmacol 252, 73-84 (2010).

3 Hartung, T. Toxicology for the twenty-first century. Nature 460, 208-212 (2009).

4 Amacher, D. E. The discovery and development of proteomic safety biomarkers for the detection of drug-induced liver toxicity. Toxicol Appl Pharmacol 245, 134-142 (2010).

5 Wetmore, B. A. \& Merrick, B. A. Toxicoproteomics: proteomics applied to toxicology and pathology. Toxicol Pathol 32, 619-642 (2004).

6 Slany, A. et al. Cell characterization by proteome profiling applied to primary hepatocytes and hepatocyte cell lines Hep-G2 and Hep-3B. J Proteome Res 9, 6-21 (2010).

7 Barrier, M. \& Mirkes, P. E. Proteomics in developmental toxicology. Reprod Toxicol 19, 291-304 (2005).

8 Gorg, A., Weiss, W. \& Dunn, M. J. Current two-dimensional electrophoresis technology for proteomics. Proteomics 4, 3665-3685 (2004).

9 Karp, N. A., Feret, R., Rubtsov, D. V. \& Lilley, K. S. Comparison of DIGE and post-stained gel electrophoresis with both traditional and SameSpots analysis for quantitative proteomics. Proteomics 8, 948-960 (2008).

10 Wilm, M. Quantitative proteomics in biological research. Proteomics 9, 4590-4605 (2009). 
11 Neilson, K. A. et al. Less label, more free: approaches in label-free quantitative mass spectrometry. Proteomics 11, 535-553 (2011).

12 Meng, Z. \& Veenstra, T. D. Targeted mass spectrometry approaches for protein biomarker verification. J Proteomics (2011).

13 Sakamoto, A. et al. Reliability and robustness of simultaneous absolute quantification of drug transporters, cytochrome P450 enzymes, and udp-glucuronosyltranferases in human liver tissue by multiplexed MRM/selected reaction monitoring mode tandem mass spectrometry with nano-liquid chromatography. J Pharm Sci 100, 40374043 (2011).

14 Aitman, T. J. et al. Progress and prospects in rat genetics: a community view. Nat Genet 40, 516-522 (2008).

15 Fountoulakis, M. et al. Two-dimensional database of mouse liver proteins: changes in hepatic protein levels following treatment with acetaminophen or its nontoxic regioisomer 3-acetamidophenol. Electrophoresis 21, 2148-2161 (2000).

16 Koen, Y. M., Gogichaeva, N. V., Alterman, M. A. \& Hanzlik, R. P. A proteomic analysis of bromobenzene reactive metabolite targets in rat liver cytosol in vivo. Chem Res Toxicol 20, 511-519 (2007).

17 Yamanaka, H., Yakabe, Y., Saito, K., Sekijima, M. \& Shirai, T. Quantitative proteomic analysis of rat liver for carcinogenicity prediction in a 28-day repeated dose study. Proteomics 7, 781-795 (2007).

18 Fielden, M. R., Brennan, R. \& Gollub, J. A gene expression biomarker provides early prediction and mechanistic assessment of hepatic tumor induction by nongenotoxic chemicals. Toxicol Sci 99, 90-100 (2007).

$19 \mathrm{Nie}, \mathrm{A}$. Y. et al. Predictive toxicogenomics approaches reveal underlying molecular mechanisms of nongenotoxic carcinogenicity. Mol Carcinog 45, 914-933 (2006).

20 Brunet, S. et al. Organelle proteomics: looking at less to see more. Trends Cell Biol 13, 629-638 (2003).

21 Lavoie, C. \& Paiement, J. Topology of molecular machines of the endoplasmic reticulum: a compilation of proteomics and cytological data. Histochem Cell Biol 129, 117-128 (2008).

22 Zgoda, V. et al. Proteomic profiles of induced hepatotoxicity at the subcellular level. Proteomics 6, 4662-4670 (2006).

23 Seliskar, M. \& Rozman, D. Mammalian cytochromes P450--importance of tissue specificity. Biochim Biophys Acta 1770, 458-466 (2007).

24 Galeva, N. \& Altermann, M. Comparison of one-dimensional and two-dimensional gel electrophoresis as a separation tool for proteomic analysis of rat liver microsomes: cytochromes P450 and other membrane proteins. Proteomics 2, 713-722 (2002).

25 Nisar, S. et al. A proteomic approach to the identification of cytochrome P450 isoforms in male and female rat liver by nanoscale liquid chromatography-electrospray ionization-tandem mass spectrometry. Drug Metab Dispos 32, 382-386 (2004)

$26 \mathrm{Jia}, \mathrm{N}$. et al. A proteomic method for analysis of CYP450s protein expression changes in carbon tetrachloride induced male rat liver microsomes. Toxicology 237, 1-11 (2007).

27 Bailey, S. M., Andringa, K. K., Landar, A. \& Darley-Usmar, V. M. Proteomic approaches to identify and characterize alterations to the mitochondrial proteome in alcoholic liver disease. Methods Mol Biol 447, 369-380 (2008).

28 Venkatraman, A. et al. Modification of the mitochondrial proteome in response to the stress of ethanoldependent hepatotoxicity. J Biol Chem 279, 22092-22101 (2004).

29 Wang, Y. et al. Plasma and liver proteomic analysis of 3Z-3-[(1H-pyrrol-2-yl)-methylidene]-1-(1piperidinylmethyl)-1,3-2H-indol-2 -one-induced hepatotoxicity in Wistar rats. Proteomics 10, 2927-2941 (2010).

30 Merrick, B. A. et al. Alterations in the rat serum proteome during liver injury from acetaminophen exposure. $J$ Pharmacol Exp Ther 318, 792-802 (2006).

31 Mathijs, K. et al. Assessing the metabolic competence of sandwich-cultured mouse primary hepatocytes. Drug Metab Dispos 37, 1305-1311 (2009).

32 Kienhuis, A. S. et al. A sandwich-cultured rat hepatocyte system with increased metabolic competence evaluated by gene expression profiling. Toxicol In Vitro 21, 892-901 (2007).

33 Kienhuis, A. S. et al. A toxicogenomics-based parallelogram approach to evaluate the relevance of coumarininduced responses in primary human hepatocytes in vitro for humans in vivo. Toxicol In Vitro 23, 1163-1169 (2009).

34 Farkas, D., Bhat, V. B., Mandapati, S., Wishnok, J. S. \& Tannenbaum, S. R. Characterization of the secreted proteome of rat hepatocytes cultured in collagen sandwiches. Chem Res Toxicol 18, 1132-1139 (2005). 
35 Zwickl, H. et al. A novel technique to specifically analyze the secretome of cells and tissues. Electrophoresis 26, 2779-2785 (2005).

36 Greenhough, S., Medine, C. N. \& Hay, D. C. Pluripotent stem cell derived hepatocyte like cells and their potential in toxicity screening. Toxicology 278, 250-255 (2010).

37 Jennen, D. G. et al. Biotransformation pathway maps in WikiPathways enable direct visualization of drug metabolism related expression changes. Drug Discov Today 15, 851-858 (2010).

38 Guyot, C. et al. Hepatic fibrosis and cirrhosis: the (myo)fibroblastic cell subpopulations involved. Int J Biochem Cell Biol 38, 135-151 (2006).

39 Wang, K., Shindoh, H., Inoue, T. \& Horii, I. Advantages of in vitro cytotoxicity testing by using primary rat hepatocytes in comparison with established cell lines. J Toxicol Sci 27, 229-237 (2002).

40 Seglen, P. O. Preparation of isolated rat liver cells. Methods Cell Biol 13, 29-83 (1976).

41 Dunn, J. C., Tompkins, R. G. \& Yarmush, M. L. Long-term in vitro function of adult hepatocytes in a collagen sandwich configuration. Biotechnol Prog 7, 237-245 (1991).

42 Kikkawa, R. et al. In vivo hepatotoxicity study of rats in comparison with in vitro hepatotoxicity screening system. J Toxicol Sci 31, 23-34 (2006).

43 Kikkawa, R., Yamamoto, T., Fukushima, T., Yamada, H. \& Horii, I. Investigation of a hepatotoxicity screening system in primary cell cultures --"what biomarkers would need to be addressed to estimate toxicity in conventional and new approaches?". J Toxicol Sci 30, 61-72 (2005).

44 Yamamoto, T., Kikkawa, R., Yamada, H. \& Horii, I. Identification of oxidative stress-related proteins for predictive screening of hepatotoxicity using a proteomic approach. J Toxicol Sci 30, 213-227 (2005).

45 Yamamoto, T., Kikkawa, R., Yamada, H. \& Horii, I. Investigation of proteomic biomarkers in in vivo hepatotoxicity study of rat liver: toxicity differentiation in hepatotoxicants. J Toxicol Sci 31, 49-60 (2006).

46 Meneses-Lorente, G. et al. A proteomic investigation of drug-induced steatosis in rat liver. Chem Res Toxicol 17, 605-612 (2004).

47 Meneses-Lorente, G. et al. Identification of early proteomic markers for hepatic steatosis. Chem Res Toxicol 19, 986-998 (2006).

48 Farkas, D. \& Tannenbaum, S. R. Characterization of chemically induced hepatotoxicity in collagen sandwiches of rat hepatocytes. Toxicol Sci 85, 927-934 (2005).

49 Rumack, B. H. Acetaminophen hepatotoxicity: the first 35 years. J Toxicol Clin Toxicol 40, 3-20 (2002).

50 Weber, L. W., Boll, M. \& Stampfl, A. Hepatotoxicity and mechanism of action of haloalkanes: carbon tetrachloride as a toxicological model. Crit Rev Toxicol 33, 105-136 (2003).

51 Tolando, R., Zanovello, A., Ferrara, R., lley, J. N. \& Manno, M. Inactivation of rat liver cytochrome P450 (P450) by $\mathrm{N}, \mathrm{N}$-dimethylformamide and N,N-dimethylacetamide. Toxicol Lett 124, 101-111 (2001).

52 Martinez, S. M. et al. Evaluation of an in vitro toxicogenetic mouse model for hepatotoxicity. Toxicol Appl Pharmacol 249, 208-216 (2010).

53 Donato, M. T., Lahoz, A., Castell, J. V. \& Gomez-Lechon, M. J. Cell lines: a tool for in vitro drug metabolism studies. Curr Drug Metab 9, 1-11 (2008).

54 Pichard, L. et al. Human hepatocyte culture. Methods Mol Biol 320, 283-293 (2006).

55 Alvergnas, M. et al. Proteomic mapping of bezafibrate-treated human hepatocytes in primary culture using twodimensional liquid chromatography. Toxicol Lett 201, 123-129 (2011).

56 Thome-Kromer, B. et al. Toward the identification of liver toxicity markers: a proteome study in human cell culture and rats. Proteomics 3, 1835-1862 (2003).

57 Choi, S. et al. Identification of toxicological biomarkers of di(2-ethylhexyl) phthalate in proteins secreted by HepG2 cells using proteomic analysis. Proteomics 10, 1831-1846 (2010)

58 Van Summeren, A. et al. Proteomics Investigations of Drug-Induced Hepatotoxicity in HepG2 Cells. Toxicol Sci 120, 109-122 (2011).

59 Pan, C., Kumar, C., Bohl, S., Klingmueller, U. \& Mann, M. Comparative proteomic phenotyping of cell lines and primary cells to assess preservation of cell type-specific functions. Mol Cell Proteomics 8, 443-450 (2009).

60 Fujii, K. et al. Database of two-dimensional polyacrylamide gel electrophoresis of proteins labeled with CyDye DIGE Fluor saturation dye. Proteomics 6, 1640-1653 (2006).

61 Wang, P. et al. Profiling of the secreted proteins during 3T3-L1 adipocyte differentiation leads to the identification of novel adipokines. Cell Mol Life Sci 61, 2405-2417 (2004). 
62 Lewis, J. A., Dennis, W. E., Hadix, J. A. \& Jackson, D. Analysis of Secreted Proteins as an in vitro Model for Discovery of Liver Toxicity Markers. J Proteome Res (2010).

63 Gao, J. et al. Identification of in vitro protein biomarkers of idiosyncratic liver toxicity. Toxicol In Vitro 18, 533-541 (2004).

64 Heijne, W. H., Stierum, R. H., Slijper, M., van Bladeren, P. J. \& van Ommen, B. Toxicogenomics of bromobenzene hepatotoxicity: a combined transcriptomics and proteomics approach. Biochem Pharmacol 65, 857-875 (2003).

65 Ruepp, S. U., Tonge, R. P., Shaw, J., Wallis, N. \& Pognan, F. Genomics and proteomics analysis of acetaminophen toxicity in mouse liver. Toxicol Sci 65, 135-150 (2002).

66 Waters, M. et al. CEBS--Chemical Effects in Biological Systems: a public data repository integrating study design and toxicity data with microarray and proteomics data. Nucleic Acids Res 36, D892-900 (2008).

67 Boitier, E. et al. A comparative integrated transcript analysis and functional characterization of differential mechanisms for induction of liver hypertrophy in the rat. Toxicol Appl Pharmacol 252, 85-96 (2011).

68 Matheis, K. A. et al. Cross-study and cross-omics comparisons of three nephrotoxic compounds reveal mechanistic insights and new candidate biomarkers. Toxicol Appl Pharmacol 252, 112-122 (2011).

69 McBurney, R. N. et al. The liver toxicity biomarker study: phase I design and preliminary results. Toxicol Pathol 37, 52-64 (2009).

70 Mathijs, K. et al. Discrimination for genotoxic and nongenotoxic carcinogens by gene expression profiling in primary mouse hepatocytes improves with exposure time. Toxicol Sci 112, 374-384 (2009).

71 de Longueville, F. et al. Use of a low-density microarray for studying gene expression patterns induced by hepatotoxicants on primary cultures of rat hepatocytes. Toxicological sciences : an official journal of the Society of Toxicology 75, 378-392, (2003).

72 Boess, F. et al. Gene expression in two hepatic cell lines, cultured primary hepatocytes, and liver slices compared to the in vivo liver gene expression in rats: possible implications for toxicogenomics use of in vitro systems. Toxicol Sci 73, 386-402 (2003).

73 Kienhuis, A. S. et al. Parallelogram approach using rat-human in vitro and rat in vivo toxicogenomics predicts acetaminophen-induced hepatotoxicity in humans. Toxicol Sci 107, 544-552 (2009).

74 Gerets, H. H. et al. Characterization of primary human hepatocytes, HepG2 cells, and HepaRG cells at the mRNA level and CYP activity in response to inducers and their predictivity for the detection of human hepatotoxins. Cell Biol Toxicol (2012).

75 Low, T. Y. et al. Quantitative and qualitative proteome characteristics extracted from in-depth integrated genomics and proteomics analysis. Cell Rep 5, 1469-1478, (2013). 


\section{CHAPTER 2}

\section{Proteomics investigations of drug-induced hepatotoxicity in HepG2 cells}

Anke Van Summeren, Johan Renes, Freek G. Bouwman, Jean-Paul Noben, Joost H. M. van Delft, Jos C. S. Kleinjans and Edwin C. M. Mariman

Toxicological Sciences 120, 109-122 (2011) 


\begin{abstract}
Unexpected hepatotoxicity is one of the major reasons of drugs failing in clinical trials. For that reason, the pharmaceutical industry is looking for new screening methods that address toxicological hazards early in the drug discovery process. 'omics approaches offers the opportunity to develop and use of in vitro models to predict hepatotoxic responses in humans. Here, proteomics techniques were used to gain further insight into the mechanistic processes of the hepatotoxic compounds. Drug-induced hepatotoxicity is mainly divided in hepatic steatosis, cholestasis or necrosis. For each class a compound was selected, respectively amiodarone, cyclosporin A and acetaminophen. The changes in protein expressions in HepG2 after exposure to these test compounds, were studied using quantitative difference gel electrophoresis (DIGE). Identification of differentially expressed proteins was performed by MaldiTOF/TOF MS and nLC-MS/MS. In this study 254 differentially expressed protein spots were detected in a 2-D proteome map from which 86 were identified. This study shows that the proteome of HepG2 cells is responsive to hepatotoxic compounds. Cyclosporin A treatment was responsible for most differentially expressed proteins and could be discriminated in the hierarchical clustering analysis. The identified differential proteins indicate that cyclosporin A induces ER stress and disturbs the ER-Golgi transport, with an altered vesicle mediated transport and protein secretion as result. Moreover, the differential protein pattern seen after cyclosporin A treatment can be related to cholestatic mechanisms. Therefore, our findings indicate that the HepG2 in vitro cell system has distinctive characteristics enabling the assessment of cholestatic properties of novel compounds at an early stage of drug discovery.
\end{abstract}




\section{INTRODUCTION}

Before a drug can be released on the market, its safety must be screened by several toxicity tests in preclinical and later clinical stages. The preclinical studies involve numerous animals, in addition they often generate false negative results, especially regarding liver toxicity ${ }^{1}$. The liver is an important target organ for toxic compounds, since it is responsible for the metabolism and elimination of chemical compounds. Unexpected hepatotoxicity is one of the main reason for withdrawal of candidate drugs from the market and emphasizes the need for novel screening methods that address toxicological hazards early in the discovery process ${ }^{2,3}$. These new screening methods are preferably in vitro test systems, to reduce the number of lab animals. By means of 'omics-technologies it is possible to identify specific hepatotoxic pathways and mechanisms. Their use in toxicological research have shown promising results and have the potential to improve the current toxicity tests ${ }^{2,4}$. Therefore, 'omics-technologies are playing an increasingly important role in the development of in vitro test systems for screening purposes.

Hepatotoxicity occurs with several symptoms which mainly fall under the following categories: hepatic steatosis, cholestasis or necrosis. Hepatic steatosis is caused by damaged mitochondrial structures, enzymes, DNA replication or transcription which can disturb the $\beta$ oxidation of lipids. Interruption of the $\beta$-oxidation cycle in hepatocytes will lead to intracellular accumulation of small lipid vesicles ${ }^{5}$. Bile transport proteins are responsible for the transport of bile salt from the hepatocyte into the bile canaliculi. Cholestasis occurs after damage of these bile transport proteins, leading to the accumulation of bile, causing secondary injury to hepatocytes ${ }^{6}$. Excessive injury to mitochondria caused by free radicals and/or toxic metabolites can cause necrosis, which is a form of premature cell death due to external factors ${ }^{7}$. Here, three drugs were used as prototypical model compounds for steatosis, cholestasis and necrosis in order to examine whether observable differences exists between these classes of hepatotoxicity, using proteomics technology. Amiodarone, which is used as an antiarrhythmic drug, has hepatic steatosis as a side effect ${ }^{8}$. Cyclosporin $A$ is a widely used immunosuppressive drug which has been shown to induce cholestasis ${ }^{9}$. The widely known and used analgesic acetaminophen is applied as a model compound for necrosis. Although acetaminophen is safe at a therapeutic dose, it is known for its toxicity at higher doses causing severe damage to the liver ${ }^{1}$.

In this study we studied the changes in protein expression of HepG2 cells exposed for 72 hours to these test compounds. The human cell line HepG2 was chosen as in vitro cell model because of its demonstrated value for screening purposes of hepatotoxic compounds ${ }^{10-13}$. For instance, Schoonen et al. classified $70 \%$ of known toxic compounds as cytotoxic when using HepG2 cells ${ }^{12,13}$. Using high content screening based on automated epifluorescence microscopy and image analysis of cells, $\mathrm{O}^{\prime}$ Brien ${ }^{11}$ was able to detect cytotoxicity in HepG2 cells with $80 \%$ sensitivity and $90 \%$ specificity. In addition, HepG2 cells has been used in many toxicogenomic studies ${ }^{14-17}$. By means of whole genome gene expression of HepG2 cells it was possible to discriminate between genotoxic and non-genotoxic carcino- 
gens ${ }^{10}$. This implies that HepG2 cells, represent a promising in vitro model for classification studies with use of toxicogenomics ${ }^{10}$. In this study we will use a proteomics approach. Previous toxicogenomics studies were mainly based on transcriptomics ${ }^{18-20}$. However, mRNA expression levels are often not parallel with the protein expression of the studied genes. To obtain a better insight into the toxicological mechanisms, it is necessary to acquire protein data by means of proteomics technologies. With proteomics not only protein expression is identified, but also post-translational modifications and protein interactions, which can give more information about mechanistic processes ${ }^{21}$. Here, the quantitative analysis of the differentially expressed proteins was done by using Difference Gel Electrophoresis (DIGE).

\section{MATERIALS AND METHODS}

\section{Chemicals}

Modified Eagle's medium (MEM) plus glutamax, sodium pyruvate, fetal calf serum (FCS), nonessential amino acids, penicillin/streptomycin, Hanks' calcium- and magnesium-free buffer were obtained from Invitrogen (Breda, The Netherlands). Dimethylsulfoxide (DMSO), Trypan blue, 3-(4,5-dimethylthiazol-2-yl)-2,5-diphenyltetrazolium bromide (MTT), acetaminophen, amiodarone, cyclosporin A (BioChemika) and $\mathrm{N}, \mathrm{N}$-dimethylformamide (anhydrous, 99.8\%) were purchased from Sigma-Aldrich (Zwijndrecht, The Netherlands). The Protein Assay Kit and the non-fat dry milk powder (NFDM) were from BioRad (Veenendaal, The Netherlands). All chemicals used for DIGE were purchased from GE Healthcare (Diegem, Belgium). The antibodies against liver carboxylesterase $1, \operatorname{Sec} 23 \mathrm{~A}$, transferrin and hnRNP A1 used for western blotting were purchased from Abcam (Cambridge, UK). The antibody against $\beta$-Actin was obtained from Santa Cruz Biotechnology (Heidelberg, Germany). The Horseradish peroxidase conjugated secondary antibodies rabbit anti mouse and swine anti rabbit were obtained from DAKO (Enschede, The Netherlands). The chemiluminescent substrate (SuperSignal CL) was purchased from Thermo Fisher Scientific (Etten-Leur, The Netherlands)

\section{Cell culture}

HepG2 cells were cultured in MEM plus glutamax containing $10 \%$ (v/v) FCS, $1 \%$ (v/v) Sodium Pyruvate, $1 \%(\mathrm{v} / \mathrm{v})$ non-essential amino acids, $2 \%(\mathrm{w} / \mathrm{v})$ penicillin and streptomycin at $37^{\circ} \mathrm{C}$ in an atmosphere containing $5 \% \mathrm{CO}_{2}$.

\section{Cytotoxicity Analysis}

HepG2 cells with a confluence of $70-80 \%$, were exposed for 72 hours to several concentrations of the test compounds (acetaminophen, amiodarone or cyclosporin A). The following concentration ranges were applied: acetaminophen 0-20 mM; amiodarone 0-100 $\mu \mathrm{M}$; cyclosporin A 0-40 $\mu \mathrm{M}$. All compounds were dissolved in DMSO and added to medium with $2 \%$ 
(v/v) FCS with a final concentration of $0.5 \%$ (v/v) DMSO. Cells incubated in the presence of $0.5 \%(\mathrm{v} / \mathrm{v})$ DMSO served as control. Cytotoxicity was determined with the MTT reduction method ${ }^{22}$ and was used to determine the $\mathrm{IC}_{20}$ of the compounds (Figure 2.1).
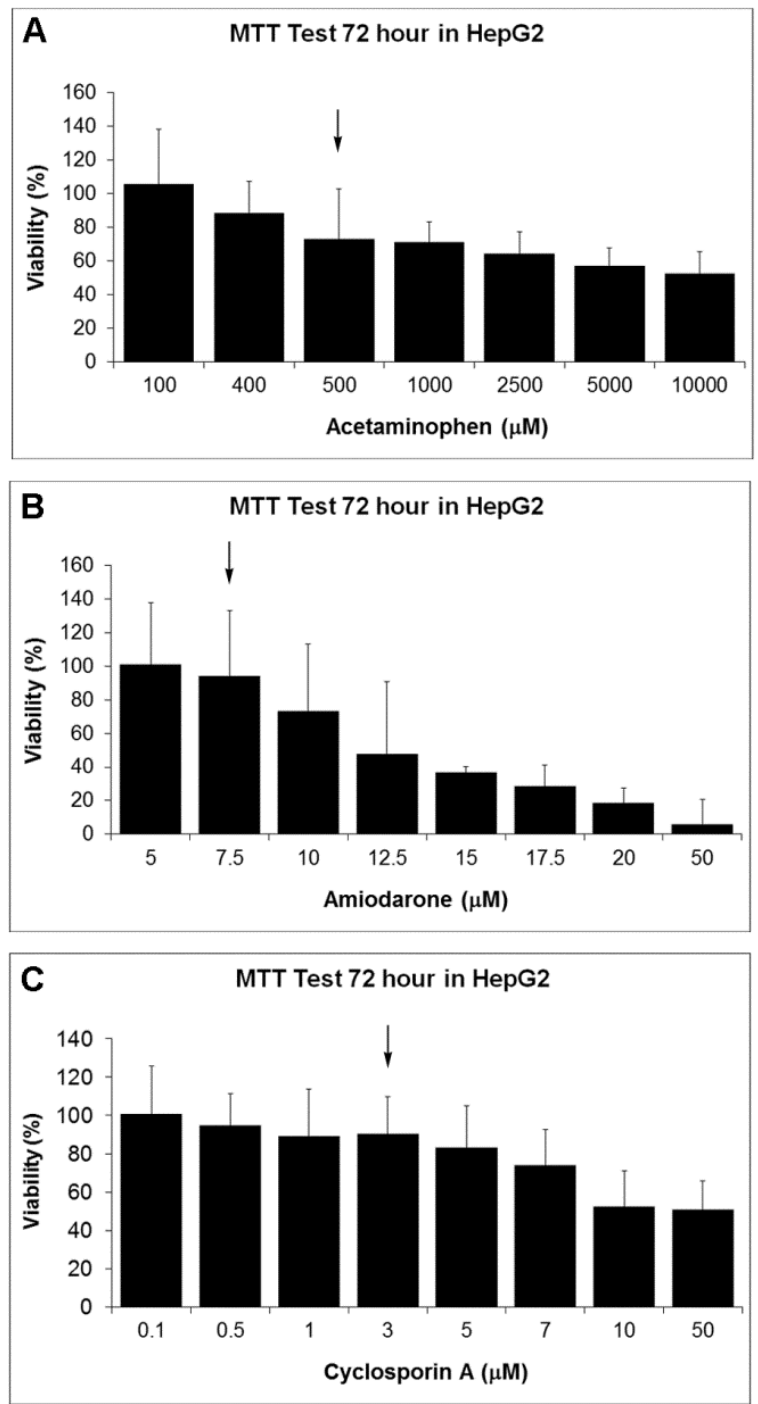

Figure 2.1: MTT test in HepG2 cells after 72 hours exposure to (A) acetaminophen, (B) amiodarone and (C) cyclosporin $\mathrm{A}$. The arrows indicate the $\mathrm{IC}_{20}$ concentration of each compound.

\section{Cell treatment}

HepG2 cells with a confluence of $70-80 \%$, were exposed for 72 hours to the $\mathrm{IC}_{20}$ concentration of the compounds determined in the cytotoxicity analysis $(0.5 \mathrm{mM}$ acetaminophen, 
7.5 $\mu \mathrm{M}$ amiodarone and $3 \mu \mathrm{M}$ cyclosporin A). Plasma levels after treatment of patients with therapeutic dosages of acetaminophen, amiodarone, cyclosporin A were found to be respectively 66-132 $\mu \mathrm{M}$. 1.6-4 $\mu \mathrm{M}$; and 0.04-0.1 $\mu \mathrm{M}^{23}$. Our experimental concentrations were chosen to be 5-10 fold higher in order to induce distinct toxicity but remained below levels causing substantial cell death.

The concentrations of the compounds in this experiment were chosen to induce an initial toxic effect without necrotizing all the cells, making it still possible to distinguish between the compounds inducing either steatosis, cholestasis or necrosis. All compounds were dissolved in DMSO and added to the medium with a final concentration of $0.5 \%(v / v)$ DMSO. Cells incubated in the presence of $0.5 \%(\mathrm{v} / \mathrm{v})$ DMSO served as control. The experiment was performed with five replicates from five independent cultures, with passage numbers between 8 and 14 . After an incubation of 72 hours the cellular proteins were isolated.

\section{Protein extraction and fluorescence dye labeling}

The cells were washed twice with ice cold PBS and harvested by scraping. The cell suspension was centrifuged at $350 \mathrm{~g}$ for $10 \mathrm{~min}$ at $4^{\circ} \mathrm{C}$. The cell pellet was dissolved in a DIGE labeling buffer 1 containing $7 \mathrm{M}$ urea, $2 \mathrm{M}$ thiourea, $4 \%$ (w/v) CHAPS and $30 \mathrm{mM}$ Tris- $\mathrm{HCl}$. This mixture was subjected to three cycles of freeze thawing with liquid nitrogen, vortexed thoroughly and centrifuged at $20000 \mathrm{~g}$ for $30 \mathrm{~min}$ at $10^{\circ} \mathrm{C}$. Supernatant was collected, aliquoted and stored at $-80^{\circ} \mathrm{C}$ until further analysis. Protein concentrations were determined with the Protein Assay Kit from Biorad (Veenendaal, the Netherlands). The proteins were labeled with CyDyes for performing the DIGE. Prior to protein labeling the $\mathrm{pH}$ of the samples was checked with a $\mathrm{pH}$ indicator strip, if necessary the $\mathrm{pH}$ was adjusted to 8.5 using $50 \mathrm{mM} \mathrm{NaOH}$. An internal standard was prepared by making a mixture with equal amounts of all the samples used in this experiment. The protein samples were labeled with Cy3 or Cy5 cyanine dyes and the internal standard with Cy2 dye, by adding 300 pmol of CyDye in $1 \mu$ l of anhydrous N,N dimethylformamide per $30 \mu \mathrm{g}$ of protein. The labeling reaction was performed for $30 \mathrm{~min}$ on ice in the dark. Afterwards the reaction was stopped by adding $10 \mathrm{mM}$ lysine followed by incubation for $10 \mathrm{~min}$ on ice in the dark. After the labeling the samples were combined. The combined samples were further adjusted to $90 \mu$ with DIGE labeling buffer 1 and afterwards further diluted with $90 \mu \mathrm{l}$ DIGE labeling buffer 2 (containing $7 \mathrm{M}$ urea, $2 \mathrm{M}$ thiourea, $4 \%$ (w/v) CHAPS, $2 \%(w / v)$ DTT and $2 \%(v / v)$ IPG buffer (pH 3-11)), to a final volume of $180 \mu \mathrm{l}$.

\section{DIGE}

The iso-electric focusing was performed with cup loading. For this, Immobiline DryStrips (24 $\mathrm{cm}, \mathrm{pH} 3-11$, non-linear) were rehydrated in $450 \mu$ Destreak $^{\mathrm{TM}}$ solution (GE Healthcare) with $0.5 \%(\mathrm{v} / \mathrm{v})$ IPG buffer ( $\mathrm{pH} 3-11)$ for 6 hours. Then the mixed samples were loaded into the cups and proteins separated by iso-electric focusing according to the following protocol: $3 \mathrm{~h}$ at $150 \mathrm{~V}, 3 \mathrm{~h}$ at $300 \mathrm{~V}, 6 \mathrm{~h}$ gradient from 600 to $1000 \mathrm{~V}, 1 \mathrm{~h} 15 \mathrm{~min}$ gradient from 1000 to 
8000 V, 3 h 45 min at 8000 V. Strips were rinsed briefly with MilliQ water and equilibrated for $15 \mathrm{~min}$ in equilibration buffer (6 M urea, $2 \%$ (w/v) SDS, $50 \mathrm{mM}$ Tris $\mathrm{HCl}$ 8.8, $30 \%$ (v/v) glycerol) with $1 \%(\mathrm{w} / \mathrm{v}) \mathrm{DTT}$, followed by 15 min incubation in equilibration buffer with $2.5 \%(\mathrm{w} / \mathrm{v})$ iodoacetamide. After each equilibration step the strips were rinsed with MilliQ water. The strips were then loaded on $12.5 \%$ SDS-polyacrylamide gels. Gels were run on the Ettan ${ }^{\mathrm{TM}}$ Dalt twelve system (GE Healthcare) at 0.5 Watt per gel at $25^{\circ} \mathrm{C}$ for 1 hour followed by 15 Watt per gel at $25^{\circ} \mathrm{C}$ until the bromophenol blue dye front almost ran off the bottom of the gels. The protein spot patterns of the three different dyes were acquired using an Ettan ${ }^{\mathrm{TM}}$ DIGE Imager laser scanner (GE Healthcare) at the excitation/emission wavelength of 488/520 nm (Cy2), $532 / 670 \mathrm{~nm}$ (Су3) and 633/670 nm (Cy5). The exposure time of the laser was chosen in a way that the protein spots had no saturated signal. For spot picking and identification a preparative gel was loaded with $150 \mu \mathrm{g}$ of the internal standard labeled with 300 pmol Cy2 and run in the same way as the analytical gels.

\section{Data Analysis}

Image analysis was performed with the DeCyder ${ }^{\mathrm{TM}} 7.0$ software (GE Healthcare), according to the manufacturer's instructions. First, protein spot detection and quantification, compared to the internal standard as a volume ratio was performed with the Differential In-gel Analysis (DIA) module. Second, protein spots on different gels were matched and statistical analysis was done with the Biological Variation Analysis (BVA) module. All statistical analysis was performed with the Extended Data Analysis (EDA) module of the DeCyder software. A one-way ANOVA test $(P \leq 0.05)$ was used to select the significant differentially abundant spots between the four groups (control, acetaminophen, amiodarone, cyclosporin A). The spots were filtered to retain those that were present in at least $80 \%$ of the spot maps. When the spots were assigned to be significant, they were carefully checked for correct matching throughout all the gels and were included in the pick list. Afterwards, the fold changes of the control and each compound were calculated based on their differences in the standardized abundance. The EDA module of the DeCyder software was also used to perform a hierarchal clustering analysis. This analysis clusters the experimental groups with similar overall protein expression together.

\section{In-gel digestion and protein identification}

Differentially expressed spots were picked from the preparative gel with the Ettan ${ }^{\mathrm{TM}}$ spot picker (GE Healthcare) and processed on a MassPREP digestion robot (Waters, Manchester, U.K.) as described before ${ }^{24}$. After the tryptic digestion $1.0 \mu \mathrm{L}$ of peptide mixture and $1.0 \mu \mathrm{L}$ of matrix solution $(2.5 \mathrm{mg} / \mathrm{mL}$-cyano-4-hydroxycinnamic acid in $50 \%$ ACN/ $0.1 \%$ TFA) were spotted automatically onto a 384 well-format target plate and air-dried for homogeneous crystallization. Mass spectra were obtained from the matrix assisted laser desorption ionization time-of-flight (MALDI-TOF/TOF) mass spectrometer (4800 MALDI TOF/TOF analyzer, Applied Biosystems) ${ }^{24}$. Samples that could not be identified via MALDI TOF/TOF MS were 
further analyzed by nano liquid chromatography tandem mass spectrometry (nLC-MSMS) on a LCQ Classic (ThermoFinnigan) as described ${ }^{25}$.

\section{Pathway and network analysis}

The differentially expressed proteins, that were identified, were further investigated by pathway analysis using MetaCore ${ }^{\mathrm{TM}}$ (GeneGo, St. Joseph, MI). For each experimental group a data set was created that contains the accession numbers of the identified proteins with their Pvalue from the Tukey's multiple comparison test and their fold changes compared to the control group. For proteins with several isoforms only the isoform with the lowest P-value was included. These data sets were imported as an Excel sheet into MetaCore ${ }^{\mathrm{TM}}$. The data sets were filtered based on the Tukeys post hoc test $(P \leq 0.05)$. The size of intersection between the subset of uploaded proteins and the proteins on all possible pathway maps in the MetaCore $^{\mathrm{TM}}$ database were computed, revealing several pathway maps. The relevant pathway maps were ranked based on their statistical significance with respect to the uploaded datasets. Further, the shortest path algorithm was used to build hypothetical networks of proteins from our experiment and proteins from the MetaCore ${ }^{\mathrm{TM}}$ database.

\section{Western blotting}

Samples with equal amount of protein ( $50 \mu \mathrm{g} / \mathrm{lane}$ ) were separated by SDS-PAGE on 4-12 \% Bis-Tris Criterion gels (Bio-Rad, Hercules, CA), at 150 V. and transferred to a $0.45-\mathrm{mm}$ nitrocellulose membranes for $90 \mathrm{~min}$ at $100 \mathrm{~V}$. After Ponceau S staining and de-staining, membranes were blocked in $5 \%$ non-fat dry milk (NFDM) in Tris-buffered saline containing $0.1 \%$ Tween 20 (TBST) for $1 \mathrm{~h}$. Thereafter, the four blots were incubated with the primary antibodies against Liver carboxylesterase 1 (1:500 dilution), Sec23A (1:500 dilution), Transferrin (1:1000 dilution) or hnRNP A1 (1:2000 dilution) in $5 \%$ NFDM-TBST overnight at $4^{\circ} \mathrm{C}$ on a shaker. Thereafter, the blots were washed three times for $10 \mathrm{~min}$ in TBST and then incubated for $1 \mathrm{~h}$ with a 1:10 000 dilution of the horseradish peroxidase conjugated secondary antibody in $5 \%$ NFDM-TBST. The blots were washed three times for $10 \mathrm{~min}$ in TBST. A CCD camera (XRSsystem, Biorad) was used to detect immunoreactive bands using chemiluminescent substrate (SuperSignal CL). The quantification was performed with the program Quantity One version 4.6.5 (Bio-Rad). $\beta$-Actin was used to standardize for the amount of protein loaded.

\section{RESULTS}

\section{DIGE analysis}

To compare the hepatotoxic effects of the drugs amiodarone, cyclosporin A and acetaminophen, cellular proteins of HepG2 cells treated for 72 hours with the test compounds or DMSO as a vehicle control were analysed using DIGE. An incubation of 72 hours was chosen because, 
in general, in vitro toxicity takes several days to express itself ${ }^{11-13}$. Also a convenient time period is necessary for the eventual translation of mRNA into proteins.

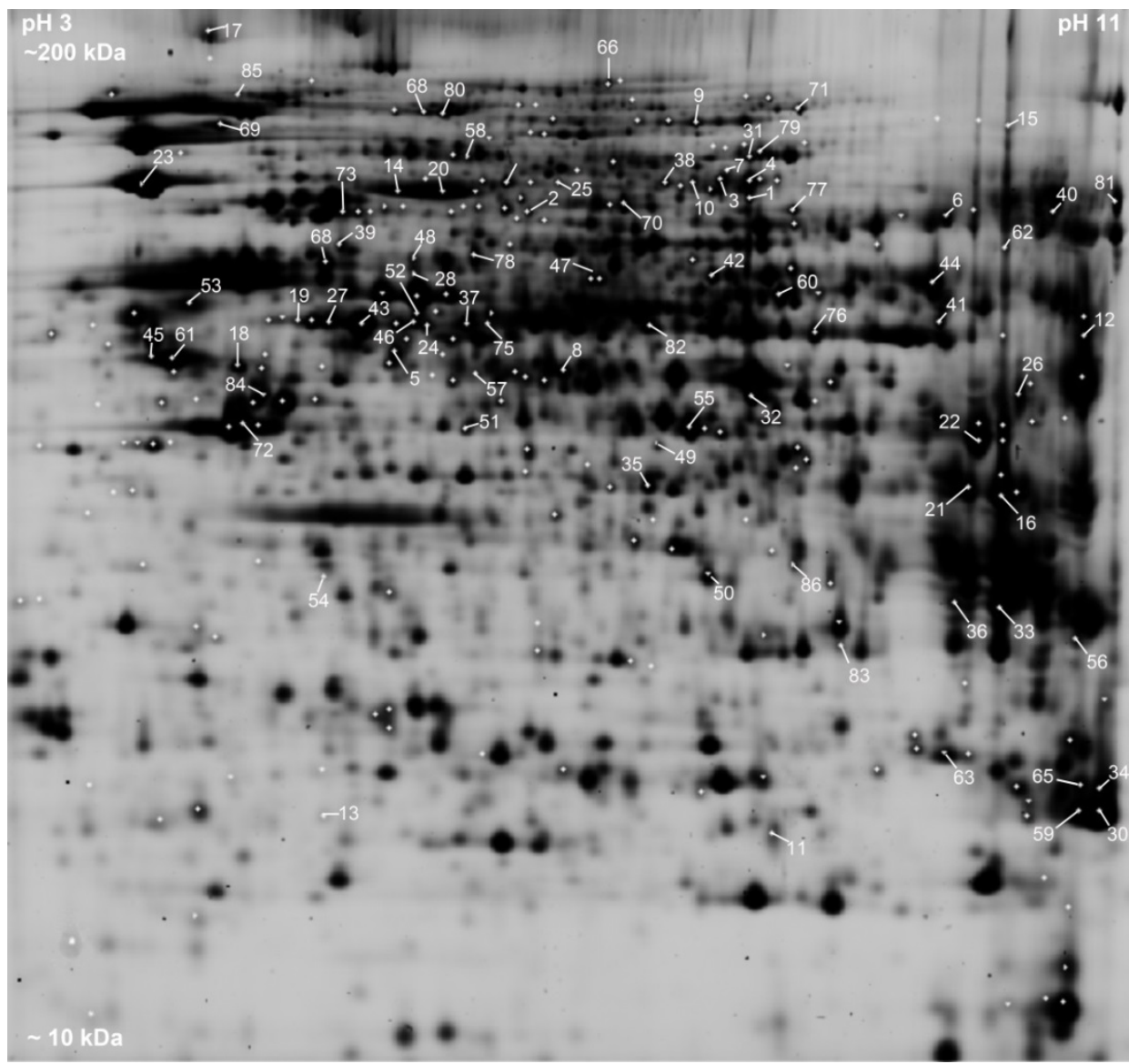

Figure 2.2: Proteome map of the differentially expressed proteins in HepG2 exposed to the hepatotoxicants Acetaminophen, Amiodarone or cyclosporin A. All the spots which are significantly differential based on one-way ANOVA (P-value $\leq 0.05$ ) are shown; the identified spots are indicated with a number which corresponds to the numbers used in Table 2.1.

In total twenty samples were obtained from this experiment (five biological replicates per group). After labeling with Cy3 or Cy5 the samples were run in ten gels together with an internal standard labeled with Cy2. The internal standard represents an average of all the samples being compared, and it is used as a normalization standard across all gels. In total 1869 spots could be matched with all the images. Out of these matched spots 254 significantly differential spots were detected with a one-way ANOVA test ( $P$ value $\leq 0.05$ ). A 2-DE map made from the master gel is presented in Figure 2.2. All the spots which are significantly differential based on one-way ANOVA ( $P$-value $\leq 0.05$ ) are shown; the identified spots are indicated with a number which corresponds to the numbers used in Table 2.1. 


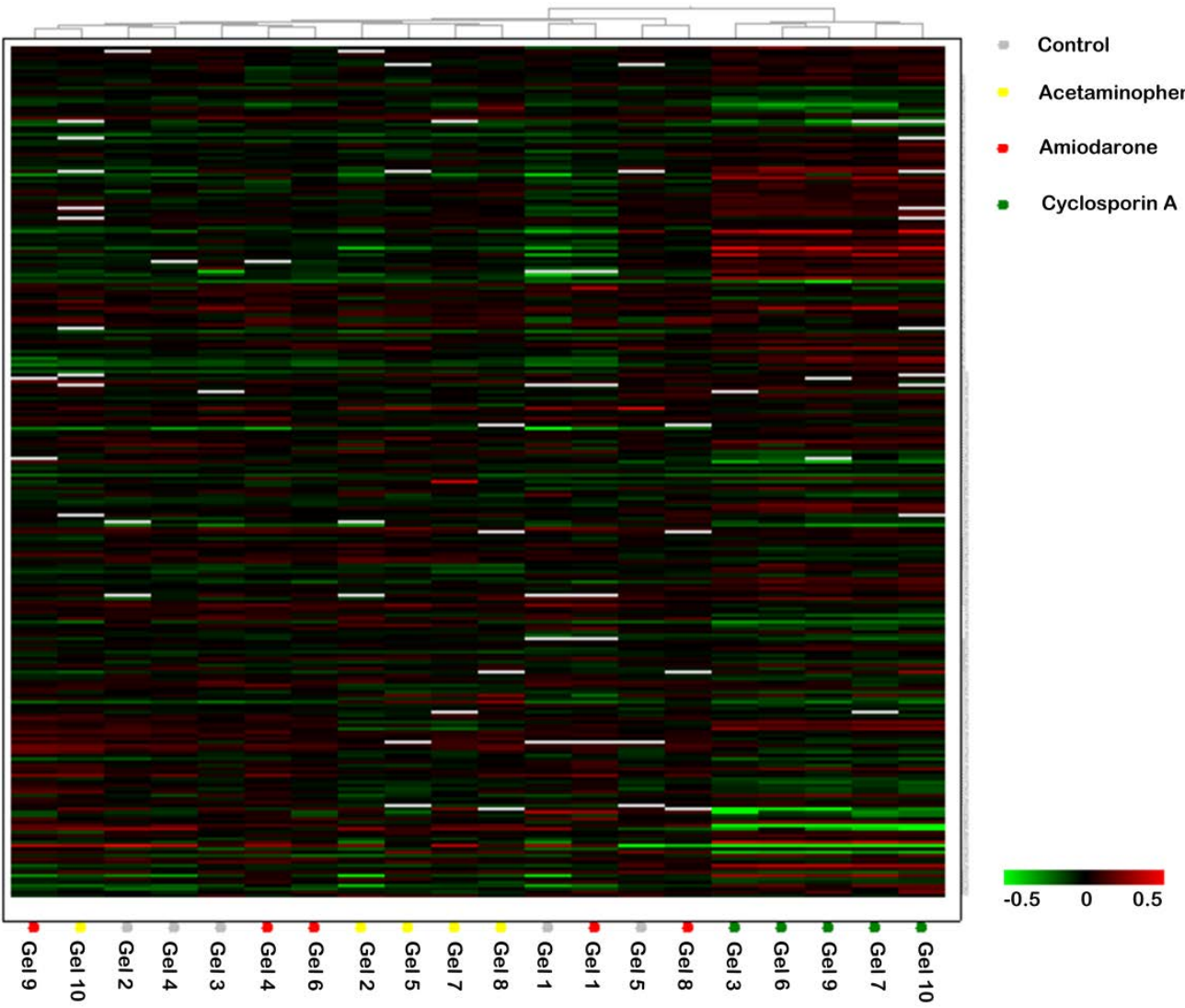

Figure 2.3: Hierarchical cluster analysis of the experimental groups (control, acetaminophen, amiodarone and cyclosporin A). The clustering is based on the log standard abundance of the 254 significant differential spots $(P$-value $\leq 0.05)$ with a hierarchal clustering algorithm in the EDA module of the Decyder software.

\section{Hierarchical clustering analysis}

The experimental groups (control, acetaminophen, amiodarone and cyclosporin A) were clustered based on the log standard abundance of the 254 differential spots with a hierarchical clustering algorithm. As shown in Figure 2.3, the spot maps of cyclosporine $A$ are distinguished the most from the other spot maps. Differences were also found between the control, acetaminophen and amiodarone although they were rather small causing them to cluster together.

\section{Protein identification}

The differential spots were included in a pick list and excised from a preparative gel. Protein identification was performed by in-gel digestion followed by MALDI-TOF/TOF and/or LC 
MS/MS analysis. Out of the 254 spots, the proteins of 86 spots were identified belonging to 69 different proteins (Table 2.1). A total of 32 spots were isoforms from 13 proteins due to post-translational modifications or processing of the protein. For spot numbers 35 and 51 two proteins for one spot were identified with LC MS/MS, both protein identifications delivering the same number of peptides. Consequently, for spot 35 and 51 it is not possible to conclude which protein is responsible for the significant change of the fold change. In Table 2.1 the spots are listed with their protein identification and their fold changes between the control and compound. The significant changes (Tukey's multiple comparison test P-value $\leq 0.05$ ) are marked with an asterisk. The results of Table 2.1 indicate that most significant differences were found between cyclosporin A and the control, which is in line with the results from the hierarchical cluster analysis. Proteins given in Table 2.1 were divided into several classes according to their function. The spot numbers were given in decreasing order according to the absolute value of their fold changes between cyclopsorin $A$ and the control. The fold changes between cyclosporin $A$ and the control revealed thirteen spots with a change $\geq 1.5$, belonging to ten proteins: serotransferrin, serum albumin, fibrinogen gamma chain, FGA Isoform 2 of fibrinogen alpha chain, protein transport protein Sec23A, alpha-enolase, elongation factor 2, keratin type I cytoskeletal 10, elongation factor 1-alpha 1, and apolipoprotein A1. Remarkably, five of these ten differential proteins are secreted proteins. The comparison of acetaminophen and the control samples showed a significant increase of liver carboxylesterase 1 with a fold change of 1.51 and of an isoform of T-complex protein subunit alpha with a fold change of 1.11. The incubation of HepG2 cells with amiodarone in HepG2 cells induced a significantly increased expression of heterogeneous nuclear ribonucleoprotein A1 with a fold change of 1.91 and of aldo-keto reductase family 1 member C1 with a fold change of 1.18. 
Table 2.1: Protein identification of differentially expressed proteins after exposure to acetaminophen, amiodarone or cyclosporin A in HepG2.

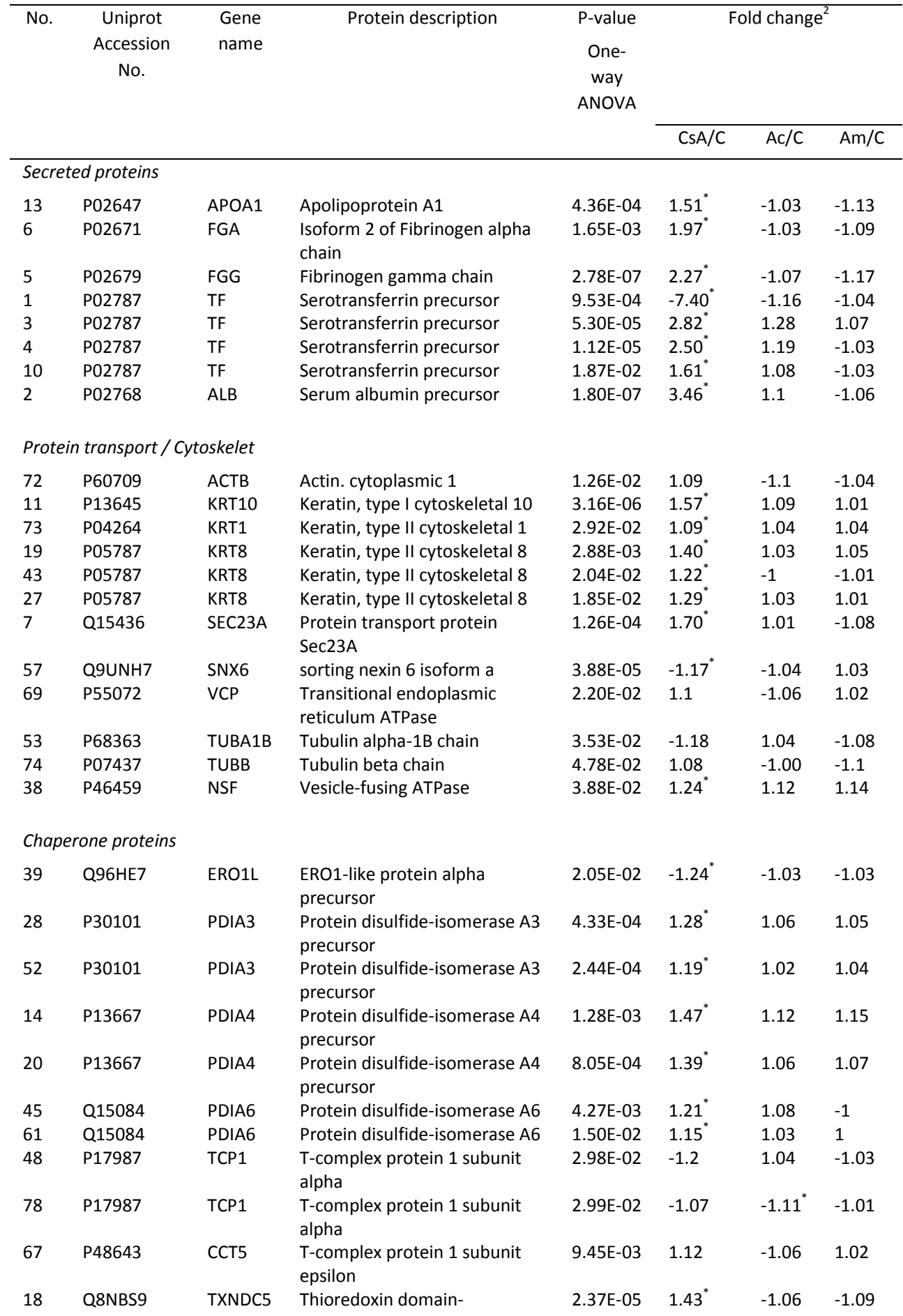




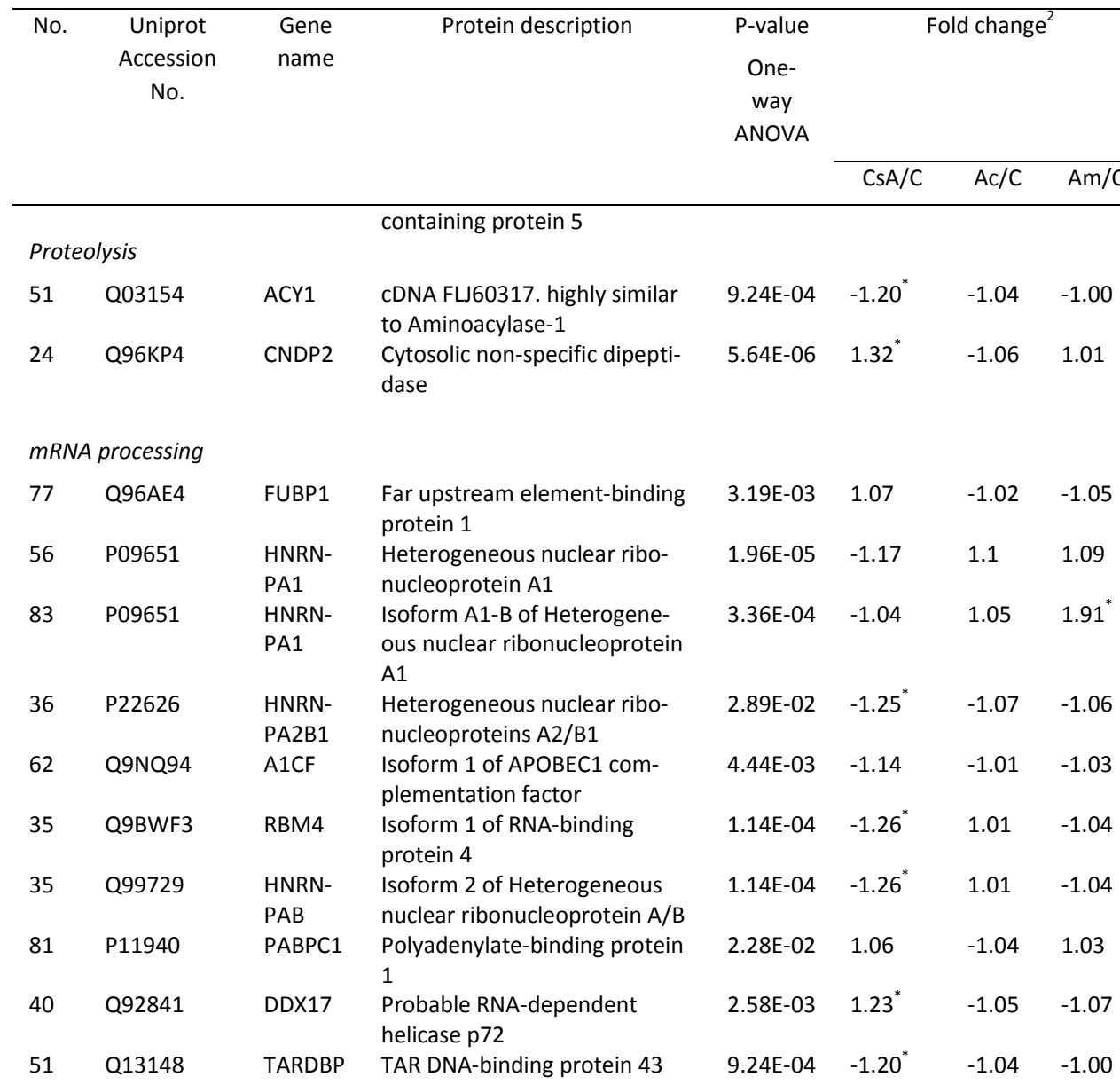

Protein biosynthesis

(1)

$12 \quad \mathrm{P} 68104$

$9 \quad \mathrm{P} 13639$

$49 \quad \mathrm{P} 49411$

EEF1A1

\section{EEF2}

TUFM

$84 \quad \mathrm{P} 60842$

31 Q92945

EIF4A1

KHSRP

$25 \quad \mathrm{P} 41250$

GARS

GARS

$65 \quad \mathrm{P} 41250$

$75 \quad \mathrm{P} 23381$

WARS

$42 \quad$ P54577

YARS

Glycolysis / gluconeogenesis

$\begin{array}{lll}32 & \text { P06733 } & \text { ENO1 } \\ 16 & \text { P04075 } & \text { ALDOA } \\ 21 & \text { P04075 } & \text { ALDOA }\end{array}$

21 P04075 ALDOA
Elongation factor 1-alpha 1

Elongation factor 2

Elongation factor Tu, mito-

chondrial

Eukaryotic initiation factor 4A-I

Far upstream element binding protein 2

GARS Glycyl-tRNA synthetase

GARS Glycyl-tRNA synthetase

Isoform 1 of Tryptophanyl-

tRNA synthetase, cytoplasmic

Tyrosyl-tRNA synthetase,

cytoplasmic

Alpha enolase

Fructose-bisphosphate al-

dolase A

Fructose-bisphosphate al-

dolase $\mathrm{A}$

$\begin{array}{llll}2.41 \mathrm{E}-02 & -1.56^{*} & -1.4 & -1.11 \\ 1.15 \mathrm{E}-03 & -1.64^{*} & -1.17 & -1.02 \\ 1.80 \mathrm{E}-02 & -1.20^{*} & -1.03 & 1.01 \\ & & & \\ 1.73 \mathrm{E}-02 & 1.04 & -1.11 & -1.03 \\ 1.92 \mathrm{E}-03 & 1.27^{*} & 1.07 & -1.02 \\ & & & \\ 1.41 \mathrm{E}-03 & 1.31^{*} & -1.04 & 1.13 \\ 2.55 \mathrm{E}-03 & 1.12 & -1.13 & 1.07 \\ 4.78 \mathrm{E}-02 & 1.08 & -1 & -1.1 \\ & & & \\ 5.81 \mathrm{E}-04 & 1.22^{*} & -1.08 & 1.14\end{array}$

$\begin{array}{llll}3.33 \mathrm{E}-02 & -1.27^{*} & -1.09 & 1.01 \\ 1.98 \mathrm{E}-04 & -1.46^{*} & -1.07 & 1.07 \\ & & & \\ 2.26 \mathrm{E}-03 & -1.37^{*} & -1.06 & 1.03\end{array}$









\begin{tabular}{ccccc}
\hline No. Uniprot & Gene & Protein description & P-value & Fold change $^{2}$ \\
Accession & name & One- & \\
No. & & way & & \\
& & ANOVA & & \\
\cline { 3 - 4 } & & & $\mathrm{CsA} / \mathrm{C} \quad \mathrm{Ac} / \mathrm{C} / \mathrm{C}$
\end{tabular}

Xenobiotic metabolic process

\begin{tabular}{|c|c|c|c|c|c|c|c|}
\hline 47 & P23141 & CES1 & $\begin{array}{l}\text { Isoform } 1 \text { of Liver carboxyles- } \\
\text { terase } 1\end{array}$ & $1.23 \mathrm{E}-02$ & 1.2 & $1.51^{*}$ & 1.27 \\
\hline 86 & Q04828 & AKR1C1 & $\begin{array}{l}\text { Aldo-keto reductase family } 1 \\
\text { member } \mathrm{C} 1\end{array}$ & $6.14 \mathrm{E}-03$ & -1.01 & 1.03 & $1.18^{*}$ \\
\hline \multicolumn{8}{|c|}{ Not listed } \\
\hline 68 & Q14697 & GANAB & $\begin{array}{l}\text { Neutral alpha-glucosidase } A B \\
\text { precursor }\end{array}$ & $1.36 \mathrm{E}-02$ & 1.11 & 1.06 & 1.02 \\
\hline 80 & Q14697 & GANAB & $\begin{array}{l}\text { Neutral alpha-glucosidase } A B \\
\text { precursor }\end{array}$ & 2.97E-02 & 1.06 & 1.07 & 1.05 \\
\hline 79 & Q92499 & DDX1 & $\begin{array}{l}\text { ATP-dependent RNA helicase } \\
\text { DDX1 }\end{array}$ & $2.53 \mathrm{E}-02$ & 1.07 & -1.01 & 1 \\
\hline 63 & P38117 & ETFB & $\begin{array}{l}\text { ETFB Isoform } 1 \text { of Electron } \\
\text { transfer flavoprotein subunit } \\
\text { beta }\end{array}$ & 6.57E-03 & 1.14 & 1.07 & 1.02 \\
\hline 54 & P62873 & GNB1 & $\begin{array}{l}\text { Guanine nucleotide-binding } \\
\text { protein } G(I) / G(S) / G(T) \text { subunit } \\
\text { beta-1 }\end{array}$ & 2.75E-02 & $1.18^{*}$ & -1.02 & -1.08 \\
\hline 58 & P14923 & JUP & JUP Junction plakoglobin & $1.36 \mathrm{E}-02$ & $1.16^{*}$ & 1.14 & 1.12 \\
\hline 15 & P55157 & MTTP & $\begin{array}{l}\text { Microsomal triglyceride trans- } \\
\text { fer protein large subunit } \\
\text { precursor }\end{array}$ & 2.97E-02 & $1.47^{*}$ & 1.03 & 1.08 \\
\hline
\end{tabular}

\footnotetext{
${ }^{1} \mathrm{P}$-value from one-way ANOVA statistical test between the four groups with each five biological replicates.

${ }_{2}$ The difference in the standardized abundance of the proteins is expressed as the fold change between the control (C) and the treated groups (T). The fold change is calculated by taking the means of standardized volume values for the protein spot in the corresponding groups $(C=$ control, $C s A=$ cyclosporin $A, A C=$ acetaminophen, $A m=a m i o d a-$ rone), values are calculated as $\mathrm{T} / \mathrm{C}$ and displayed in the range of +1 to $+\infty$ for increases in expression and calculated as $-\mathrm{C} / \mathrm{T}$ and displayed in the range of $-\infty$ to -1 for decreased expression.

${ }^{*}$ Indicates significant fold changes $(\mathrm{P} \leq 0.05)$ between the control and the treated group, calculated with a multiple comparison test.
}

\section{Pathway and network analysis}

Metacore ${ }^{\mathrm{TM}}$ Pathway analysis was performed on the differentially expressed proteins. First, the data sets were filtered based on the Tukey's post hoc test $(P \leq 0.05)$; here only cyclosporin A delivered a list of significantly differential proteins, which is in line with the results from the hierarchal clustering analysis. Therefore we decided to focus on the data set of cyclosporin $A$ in pathway analysis. Pathway maps with a $\mathrm{P} \leq 0.05$ were considered as relevant and are shown in Table 2.2. The affected pathways involve glycolysis/gluconeogenesis, pyruvate metabolism, HDL metabolism, cholesterol and protein trafficking from the endoplasmic reticulum (ER) to the Golgi apparatus. 
Table 2.2: Pathway maps based on pathway analysis from differential expressed proteins in HepG2 after 72 hours incubation with $3 \mu \mathrm{M}$ cyclosporin $\mathrm{A}$, as performed by Metacore ${ }^{\mathrm{TM}}$.

\begin{tabular}{|c|c|c|c|c|}
\hline \multirow{2}{*}{$\begin{array}{l}\text { Map } \\
\text { Glycolysis and gluconeogenesis } \\
\text { (short map) }\end{array}$} & \multirow{2}{*}{$\begin{array}{l}\text { Map Folders } \\
\text { Metabolic maps/Metabolic maps (common } \\
\text { pathways)/ Carbohydrates metabolism }\end{array}$} & \multirow{2}{*}{$\begin{array}{l}\text { p-value } \\
3.782 E-05\end{array}$} & \multicolumn{2}{|c|}{ Objects } \\
\hline & & & 6 & 37 \\
\hline Glycolysis and gluconeogenesis p.3 & $\begin{array}{l}\text { Metabolic maps/Metabolic maps (common } \\
\text { pathways)/ Carbohydrates metabolism }\end{array}$ & $1.126 \mathrm{E}-04$ & 4 & 15 \\
\hline $\begin{array}{l}\text { Glycolysis and gluconeogenesis p.3 } \\
\text { / Human version }\end{array}$ & $\begin{array}{l}\text { Metabolic maps/Organism-specific metabol- } \\
\text { ic maps for Mouse, Rat and Hu- } \\
\text { man/Carbohydrates metabolism }\end{array}$ & $1.126 \mathrm{E}-04$ & 4 & 15 \\
\hline $\begin{array}{l}\text { Transcription_Role of Akt in hypox- } \\
\text { ia induced HIF1 activation }\end{array}$ & $\begin{array}{l}\text { Protein function/Kinases Protein func- } \\
\text { tion/Transcription factors Regulatory pro- } \\
\text { cesses/Hypoxia response }\end{array}$ & $1.068 \mathrm{E}-03$ & 4 & 26 \\
\hline Pyruvate metabolism & $\begin{array}{l}\text { Metabolic maps/Metabolic maps (common } \\
\text { pathways)/ Carbohydrates metabolism }\end{array}$ & $1.103 \mathrm{E}-02$ & 3 & 26 \\
\hline $\begin{array}{l}\text { Cholesterol and Sphingolipids } \\
\text { transport / Recycling to plasma } \\
\text { membrane in lung (normal and CF) }\end{array}$ & Disease maps/Lung Diseases/Cystic Fibrosis & $1.623 \mathrm{E}-02$ & 2 & 11 \\
\hline $\begin{array}{l}\text { Normal wtCFTR traffic / ER-to- } \\
\text { Golgi }\end{array}$ & Disease maps/Lung Diseases/Cystic Fibrosis & $2.249 \mathrm{E}-02$ & 2 & 13 \\
\hline $\begin{array}{l}\text { Delta508-CFTR traffic / ER-to-Golgi } \\
\text { in CF }\end{array}$ & Disease maps/Lung Diseases/Cystic Fibrosis & $2.249 \mathrm{E}-02$ & 2 & 13 \\
\hline $\begin{array}{l}\text { Chemotaxis_Lipoxin inhibitory } \\
\text { action on } \mathrm{fMLP} \text {-induced neutrophil } \\
\text { chemotaxis }\end{array}$ & $\begin{array}{l}\text { Protein function/Cyto/chemokines Regula- } \\
\text { tory processes/Cell adhesion Regulatory } \\
\text { processes/Chemotaxis Regulatory process- } \\
\text { es/Cytoskeleton remodeling Regulatory } \\
\text { processes/Immune response }\end{array}$ & $2.297 \mathrm{E}-02$ & 3 & 34 \\
\hline $\begin{array}{l}\text { Cytoskeleton remodeling_Keratin } \\
\text { filaments }\end{array}$ & $\begin{array}{l}\text { Regulatory processes/Cytoskeleton remod- } \\
\text { eling }\end{array}$ & $2.673 \mathrm{E}-02$ & 3 & 36 \\
\hline $\begin{array}{l}\text { Blood coagulation_Blood coagula- } \\
\text { tion }\end{array}$ & Regulatory processes/Blood coagulation & $2.673 \mathrm{E}-02$ & 3 & 36 \\
\hline Niacin-HDL metabolism & $\begin{array}{l}\text { Metabolic maps/Metabolic maps (common } \\
\text { pathways)/Vitamin and cofactor metabo- } \\
\text { lism }\end{array}$ & $3.343 E-02$ & 2 & 16 \\
\hline $\begin{array}{l}\text { Protein folding Membrane traffick- } \\
\text { ing and signal transduction of G- } \\
\text { alpha (i) heterotrimeric G-protein }\end{array}$ & $\begin{array}{l}\text { Protein function/G-proteins Regulatory } \\
\text { processes/Transport }\end{array}$ & $4.603 \mathrm{E}-02$ & 2 & 19 \\
\hline
\end{tabular}

The imported data sets contained the accession numbers of the identified proteins with their P-value from the Tukey's multiple comparison test and their fold change compared to the control group. The relevant pathway maps are filtered and ranked based on their statistical significance $(P \leq 0.05)$. The network analysis performed by means of the shortest path algorithm shows a hypothetical network built on proteins from our experiment and proteins from the MetaCore ${ }^{\mathrm{TM}}$ database (Figure 2.3). 


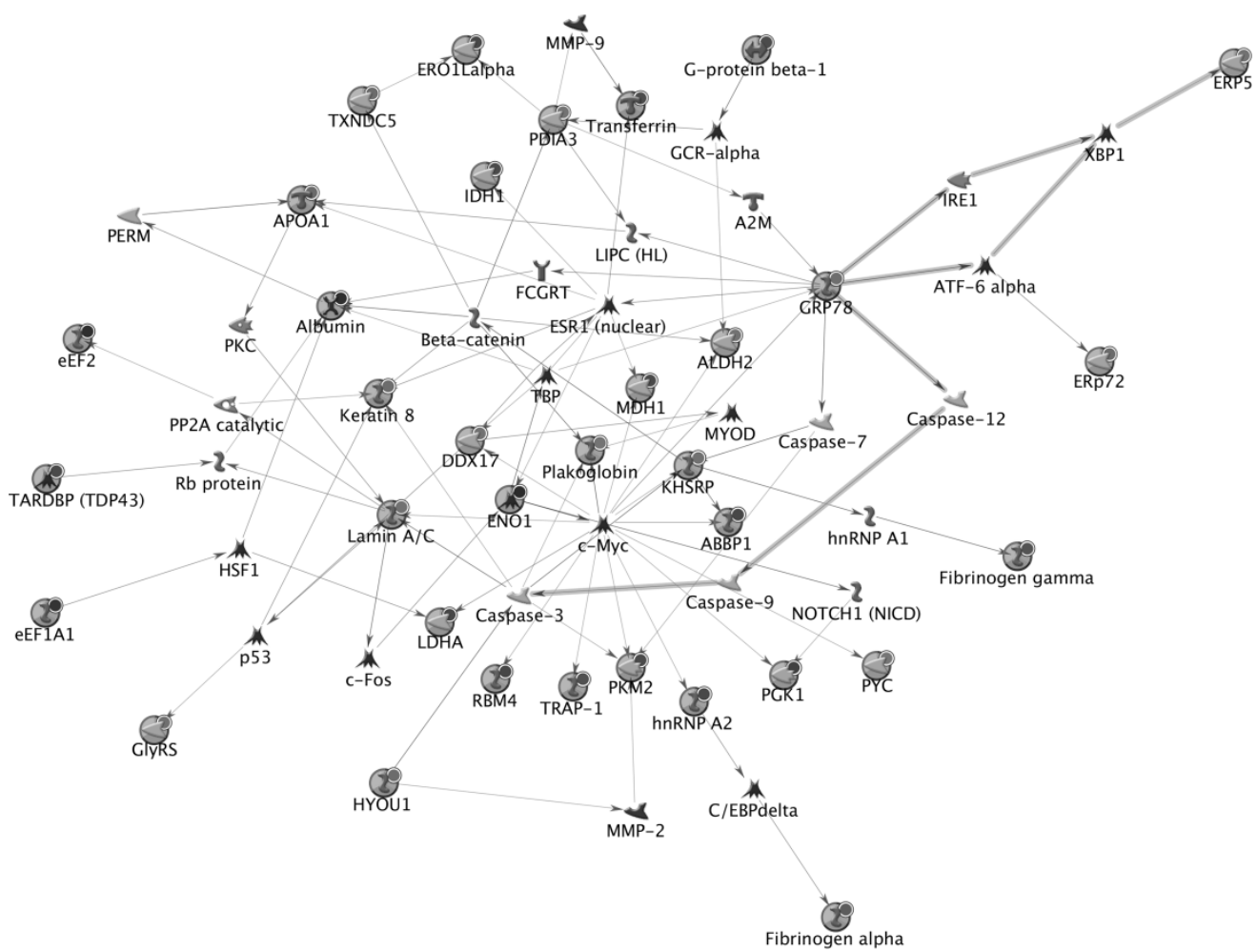

Figure 2.3: Protein networks associated with the proteins differentially expressed by HepG2 cells after 72 hours incubation with $3 \mu \mathrm{M}$ cyclosporin A. The network was generated by shortest path algorithm of MetaCore ${ }^{\mathrm{TM}}$ using the list of differentially expressed proteins identified by DIGE/MS analysis. Individual proteins are represented as nodes, and the different shapes of the nodes represent the functional class of the proteins. The edges define the relationships of the nodes: the arrowheads indicate the direction of the interaction.

\section{Western blotting}

To confirm our findings obtained with the DIGE analysis, western blotting was performed for four significantly changed proteins. The DIGE results revealed a significant differential expression of several protein spots belonging to serotransferrin upon exposure to cyclosporin $A$ (Table 2.1, spot no 1, 3, 4 and 10) Most of these spots were up-regulated while one spot was down-regulated. This indicates the presence of posttranslational modifications. The western blot analysis showed a trend $(P=0.069)$ for a cyclosporin $A$ induced expression of serotransferrin (Figure 2.4A). On the other hand, with acetaminophen a significantly increased expression of serotransferrin was observed with the western blot. This agrees with the trend for increased expression found with the DIGE analysis.

The DIGE analysis showed a significant up-regulation of protein transport protein Sec23A by cyclosporin (spot no. 7), which was confirmed by our western blot data (Figure 2.4B, 
$P=0.00044)$. Expression levels of Heterogenous nuclear ribonucleoprotein A1 were not found to be significantly changed with the western blot analysis (Figure 2.4C). However, although not significant, with amiodarone an increased expression of this protein was observed which is in line with our DIGE analysis (spot no. 83). With respect to liver caboxylesterase 1, the DIGE analysis showed an increased expression of this protein with all three drugs which was significant for acetaminophen. The western blot showed a significantly increased expression for all three drugs with P-values of $0.00024 ; 0.0082 ; 0.00022$ for acetaminophen, amiodarone and cyclosporin A, respectively (Figure 2.4D). Overall, western blot results were well in line with the results from DIGE-analysis.
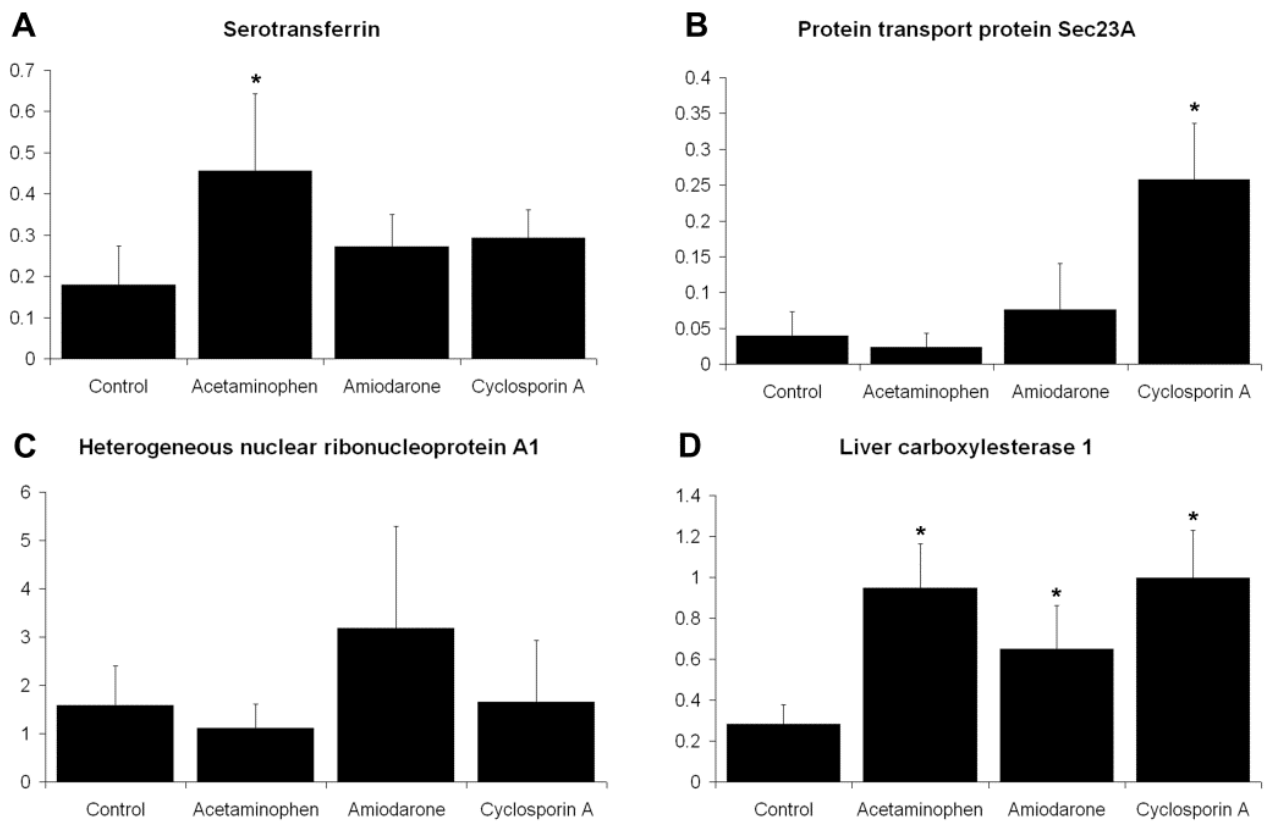

Figure 2.4: Expression differences by western blotting of 4 different proteins from HepG2 cells treated with amiodarone, CyclosporinA or acetaminophen. (A) Serotransderrin, (B) Protein transport protein Sec23A, (C) Heterogeneous nuclear ribonucleoprotein A1 (D) Liver carboxyl esterase 1.

\section{DISCUSSION}

In this study three model compounds (amiodarone, cyclosporin A and acetaminophen) for either steatosis, cholestasis or necrosis were investigated in order to examine whether distinct differences exist between these classes of hepatotoxicity. We studied the changes in protein expressions in HepG2 cells after 72 hours exposure to these test compounds. By means of hierarchical clustering analyses, it was possible to distinguish cyclosporin A from amiodarone and acetaminophen based on their hepatotoxic effects reflected in the proteome of HepG2 cells. On the other hand, the experimental set-up was not able to make an ade- 
quate differentiation between amiodarone, acetaminophen and the control samples. This despite the fact that the concentrations of the compounds used in this study caused $20 \%$ of cell death detected with a MTT test (Figure 2.1). Probably the differences in cytotoxic effects between these compounds are relatively small, and not detectable with the applied DIGE method. Compared to primary hepatocytes, HepG2 cells have a low expression of certain cytochrome P450 (CYP450) enzymes ${ }^{26}$. CYP450 enzymes are known to be responsible for the biotransformation of acetaminophen in $\mathrm{N}$-acetyl-p-benzoquinoneimine which is the main metabolite responsible for the toxicity of acetaminophen ${ }^{27}$. Amiodarone is extensively metabolized in the liver by CYP3A4 to its toxic mono-N-desethyl and di-N-desethyl metabolites ${ }^{28}$. For acetaminophen and amiodarone a low expression of CYP450 enzymes can lead to less toxic metabolites and therefore only a relatively small effect can be observed in HepG2.

After incubation with acetaminophen, HepG2 cells show an increased expression of liver carboxylesterase 1 (CES1). Carboxylesterases are categorized as phase I drug metabolizing enzymes which are responsible for the hydrolysis of ester- and amide bond-containing drugs and pro-drugs ${ }^{29}$. Other studies detected elevated serum carboxylesterases in patients suffering from necrotizing liver diseases and liver damage as a consequence of overdoses of acetaminophen ${ }^{30}$. These studies even suggest that carboxylesterases may serve as an indicator of liver damage ${ }^{30}$. Therefore, its differential expression indicates that acetaminophen did induce hepatotoxicity in our experiment.

Amiodarone induced an increased expression of heterogeneous nuclear ribonucleoprotein A1 (HNRNP A1). In a previous study it was shown that HNRNP A1 binds with the transcript of Cyp2a5 and protects it from degradation ${ }^{31}$.

Further, in the amiodarone treated cells, a significantly increased expression of aldoketoreductase family $1 \mathrm{C} 1$ is shown. Aldo-ketoreductases are Phase I drug metabolizing enzymes for a variety of carbonyl containing drugs. They are known to detoxify reactive aldehydes formed from exogenous toxicants, such as aflatoxin, endogenous toxicants, and those formed from the breakdown of lipid peroxides ${ }^{32}$. So, an increased expression of aldoketoreductase can indicate an excess amount of lipid peroxides, which need to be broken down by the aldo-ketoreductase. Hence, differential expression of aldo-ketoreductase $1 \mathrm{C} 1$ may play a role in the development of steatosis induced by amiodarone.

cyclosporin $A$ is a strong immunosuppressant that inhibits both lymphokine release and subsequent activation of cytotoxic $T$ cells, but induces cholestasis as a side effect ${ }^{33}$. The mechanism for cholestasis development upon cyclosporin A treatment can be explained by cyclosporin $A$ being a competitive inhibitor of the bile salt export pump (ABCB11), multidrug resistance protein 2 ( $A B C C 2$ ) and P-glycoprotein ( $A B C B 1$ ) in canalicular membrane vesicles ${ }^{34,35}$. These ATP Binding Cassette transporters ( $A B C$ transporters) are responsible for the secretion of bile components into the bile canaliculus ${ }^{36}$. Therefore, their blocking inhibits bile secretion, thus resulting in cholestasis ${ }^{37}$.

The protein expression in HepG2 cells treated with cyclosporin A, reveals a remarkably high differential expression of secreted proteins is shown such as: serum albumin, ApoA1, sero- 
transferrin and fibrinogen. Other studies have reported an inhibition of protein secretion related to cyclosporin A. For instance, Lodish and Kong ${ }^{38}$ have reported an inhibited secretion of transferrin caused by cyclosporin A, where our dataset show an increase of cellular serotransferrin. An inhibited secretion was ascribed to the disturbed activity of the cyclophilins after binding with cyclosporin A. Cyclophillins are peptidyl-prolyl-trans isomerases, enzymes that accelerate or slow down steps in the folding of proteins. Therefore, inhibited activity of cyclophillin can be responsible for an inhibited folding of transferrin and other secreted proteins ${ }^{38}$. However the amounts of cyclophillin binding sites that are blocked at therapeutically concentrations are estimated at $1-2 \%$ of the total binding sites, so probably this is not the only mechanism responsible for an inhibited secretion ${ }^{39}$. Kockx et al. ${ }^{40}$ have shown an inhibited secretion and degradation of ApoE independent from ABCA1 transporter inhibition mediated by cyclosporin A. By means of fluorescent staining, an inhibited ApoE trafficking from the ER to the Golgi apparatus was shown. This was explained by an affected vesicle transport from the ER to the Golgi apparatus with a link to calcineurin inhibition ${ }^{40}$. Here we report differential expression of the sec23A transport protein, vesicle-fusing ATPase, tubulin alpha-1B chain, actin cytoplasmic 1, transitional endoplasmic reticulum ATPase and sorting nexin- 6 which are important proteins for vesicle mediated transport from the ER to the Golgi apparatus. Furthermore, several chaperone proteins were found differentially expressed, for example protein disulfide isomerases $A 3, A 4$ and $A 6$, thioredoxin domain-containing protein 5 , endoplasmic oxidoreductin-1-like protein, T-complex protein 1 subunit alpha, which may indicate ER stress. Therefore cyclosporin A probably induces ER stress, with an altered chaperone activity together with disturbed protein transport resulting in a decreased protein secretion. In the electron microcopy study of Ryffel et al. ${ }^{41}$ cellular swelling, dilatation of the endoplasmic reticulum, and the presence of lipid droplets and giant mitochondria was observed after a cyclosporin A treatment. This observation probably visualized both a disturbed vesicle mediated transport with ER stress together with a mitochondrial dysfunction. A disturbance of the calcium homeostasis could be a common cause for the dysfunction of both ER and mitochondria. It is well known that functional interactions between the regulation of calcium stores in mitochondria and ER are important for the communication between these organelles in calcium homeostasis ${ }^{42,43}$. A perturbation of intracellular Ca-signaling with cyclosporin A was reported in other studies ${ }^{44,45}$. The ER contains a pool of calcium ions, which is essential for the translocation, folding, glycosylation, disulphide bonding and sorting of secreted proteins ${ }^{46}$. Bonilla et al. showed that the depletion of $\mathrm{Ca}^{2+}$ from the ER indeed disturbs the efficiency of protein folding 47 .

Bile acids are mainly transported by ATP driven transport proteins, but when the trans cellular flux and biliary excretion of bile salts increases, vesicle mediated secretion of bile will also play an important role in hepatocytes ${ }^{48}$. As mentioned before, cyclosporin $A$ is an inhibitor of $A B C B 11, A B C C 2$ and $A B C B 1$ and consequently inhibits the secretion of bile into the bile canaliculus, causing cholestasis ${ }^{34}$. Nevertheless, it is possible that besides vesicle mediated protein transport, also the vesicle mediated bile transport is affected by cyclosporin A, promoting 
the development of cholestasis. This was also proposed by Roman et al. ${ }^{49}$ after their experiment indicated an inhibition of hepatocellular vesicular transport by cyclosporin $A$ in rats.

The ABCA1 transporter protein is also blocked by cyclosporin $A^{50}$. This transporter is important for the ApoA1/HDL generation. Endogenous ApoA1 is secreted by the HepG2 cells and then interacts with cellular ABCA1 to generate $\mathrm{HDL}^{51}$. The inhibition of the ABCA1 transporter will result in reduced lipid secretion and low HDL plasma levels ${ }^{40,52,53}$. An increased expression of ApoA1 in the cellular protein fraction as seen in our study is probably the result of a reduced secretion of ApoA1. This reduced secretion probably is due to ER stress and will have a further lowering effect on the HDL plasma levels.

The findings of the cyclosporin A dataset are confirmed by pathway analysis, done with Metacore $^{\mathrm{TM}}$. The glycolysis/gluconeogenesis and pyruvate metabolism, were assigned as differential pathways. The affecting of these pathways signifies a disturbed metabolic activity of the cells by cyclosporin A treatment. Also pathways related to cystic fibrosis were found to be altered significantly. Cystic fibrosis is often observed together with cholestatic liver disease ${ }^{54}$. Cystic fibrosis is a genetic disorder with a mutation of the cystic fibrosis membrane conductance regulator (CFTR), that is also an $A B C$ transport protein ( $A B C C 7)^{55}$. Additionally, the cystic fibrosis disturbed pathways involve the ER-Golgi trafficking, emphasizing that ER stress and affected ER-Golgi trafficking are core-elements of cyclosporin A-induced cholestasis.

Together with pathway analysis a hypothetical network was built from the differential expressed proteins of our experiment and proteins from the MetaCore ${ }^{\mathrm{TM}}$ database. The nodes from this generated network involve several apoptotic proteins, like the members 3, 9 and 12 of the Caspase family which play an essential role in apoptosis. ER related proteins GRP78, ERP5 and HYOU1 in this network may indicate that this apoptotic reaction is a response of ER stress. Centrally positioned in this network is the C-Myc protein; this protein is a transcription factor that plays a role in cell apoptosis but is also involved in cell cycle progression and cellular transformation.

Another remarkable node from the network is estrogen receptor ESR1, which is a nuclear hormone receptor. It is known that estrogens can cause cholestasis in susceptible women during pregnancy, after administration of oral contraceptives or during a postmenopausal therapy ${ }^{56}$. Yamamoto et al. ${ }^{57}$ showed that the synthetic estrogen 17 -ethynylestradiol induces liver damage by activating the ESR1 signalling pathway ${ }^{57}$. Moreover, ESR1 repressed the expression of bile acid and cholesterol transporters in the liver. In line with this, biliary secretions of both bile acids and cholesterol were markedly decreased in 17-ethynylestradiol treated wild-type mice but not in the estradiol treated ESR1 knockout mice ${ }^{57}$. The nodal position of ESR1 which is a main receptor for estradiol, suggests that cyclosporin A- and estradiolinduced cholestasis rely on similar mechanisms.

Similar in vivo and in vitro proteome studies, revealed the differential expression of several proteins related to oxidative stress and mitochondrial metabolism were found differentially expressed due to drug-induced hepatotoxicity ${ }^{58-60}$. The results of these studies follow the 
same trend of our findings though our study could identify proteins related to ER stress and secreted proteins which may be specific for cyclosporin A treatment.

In summary, based on a mechanistic proteome analysis, we showed that with HepG2 cells it is possible to distinguish modes-of-action of the cholestatic compound cyclosporin A from the other hepatotoxic compounds amiodarone and acetaminophen. We identified several differential proteins related to cyclosporin A induced ER stress and the ER-Golgi transport, which may alter vesicle mediated transport and protein secretion. Several findings explicate that the differential protein expression pattern seen with cyclosporin $A$, is related to cholestatic mechanisms. Therefore the HepG2 in vitro cell system probably has distinctive characteristics in order to detect cholestasis at an early stage of drug discovery. Additional investigations with other cholestatic compounds are required for a further generalization of our present results.

\section{ACKNOWLEDGMENTS}

We thank Erik Royackers from the Biomedical Research Institute of Hasselt University for his technical support of the LC MS/MS analysis. 


\section{REFERENCES}

1 Murray, K. F., Hadzic, N., Wirth, S., Bassett, M. \& Kelly, D. Drug-related hepatotoxicity and acute liver failure. $J$ Pediatr Gastroenterol Nutr 47, 395-405 (2008).

2 Amacher, D. E. The discovery and development of proteomic safety biomarkers for the detection of drug-induced liver toxicity. Toxicol Appl Pharmacol 245, 134-142 (2010).

3 Lee, W. M. Drug-induced hepatotoxicity. N Engl J Med 349, 474-485 (2003).

4 Blomme, E. A., Yang, Y. \& Waring, J. F. Use of toxicogenomics to understand mechanisms of drug-induced hepatotoxicity during drug discovery and development. Toxicol Lett 186, 22-31 (2009).

5 Fromenty, B. \& Pessayre, D. Inhibition of mitochondrial beta-oxidation as a mechanism of hepatotoxicity. Pharmacol Ther 67, 101-154 (1995).

6 Pauli-Magnus, C. \& Meier, P. J. Hepatobiliary transporters and drug-induced cholestasis. Hepatology 44, 778-787 (2006).

7 Nelson, S. D. Mechanisms of the formation and disposition of reactive metabolites that can cause acute liver injury. Drug Metab Rev 27, 147-177 (1995).

8 Fromenty, B. et al. Amiodarone inhibits the mitochondrial beta-oxidation of fatty acids and produces microvesicular steatosis of the liver in mice. J Pharmacol Exp Ther 255, 1371-1376 (1990).

9 Rotolo, F. S., Branum, G. D., Bowers, B. A. \& Meyers, W. C. Effect of cyclosporine on bile secretion in rats. Am J Surg 151, 35-40 (1986).

10 Jennen, D. G. et al. Comparison of HepG2 and HepaRG by whole-genome gene expression analysis for the purpose of chemical hazard identification. Toxicol Sci 115, 66-79 (2010).

11 O'Brien, P. J. et al. High concordance of drug-induced human hepatotoxicity with in vitro cytotoxicity measured in a novel cell-based model using high content screening. Arch Toxicol 80, 580-604 (2006).

12 Schoonen, W. G., de Roos, J. A., Westerink, W. M. \& Debiton, E. Cytotoxic effects of 110 reference compounds on HepG2 cells and for 60 compounds on HeLa, ECC-1 and CHO cells. II mechanistic assays on NAD(P)H, ATP and DNA contents. Toxicol In Vitro 19, 491-503 (2005).

13 Schoonen, W. G., Westerink, W. M., de Roos, J. A. \& Debiton, E. Cytotoxic effects of 100 reference compounds on Hep G2 and HeLa cells and of 60 compounds on ECC-1 and CHO cells. I mechanistic assays on ROS, glutathione depletion and calcein uptake. Toxicology in vitro : an international journal published in association with BIBRA 19, 505-516, (2005).

14 Hockley, S. L. et al. Interlaboratory and interplatform comparison of microarray gene expression analysis of HepG2 cells exposed to benzo(a)pyrene. Omics 13, 115-125 (2009).

15 Hong, Y., Muller, U. R. \& Lai, F. Discriminating two classes of toxicants through expression analysis of HepG2 cells with DNA arrays. Toxicol In Vitro 17, 85-92 (2003).

16 Burczynski, M. E. et al. Toxicogenomics-based discrimination of toxic mechanism in HepG2 human hepatoma cells. Toxicol Sci 58, 399-415 (2000).

17 Iguchi, K. et al. Identification of differentially expressed genes in hepatic HepG2 cells treated with acetaminophen using suppression subtractive hybridization. Biol Pharm Bull 28, 1148-1153 (2005).

18 Harris, A. J., Dial, S. L. \& Casciano, D. A. Comparison of basal gene expression profiles and effects of hepatocarcinogens on gene expression in cultured primary human hepatocytes and HepG2 cells. Mutat Res 549, 79-99 (2004).

19 Kienhuis, A. S. et al. A toxicogenomics-based parallelogram approach to evaluate the relevance of coumarininduced responses in primary human hepatocytes in vitro for humans in vivo. Toxicol In Vitro 23, 1163-1169 (2009).

20 Waring, J. F., Ciurlionis, R., Jolly, R. A., Heindel, M. \& Ulrich, R. G. Microarray analysis of hepatotoxins in vitro reveals a correlation between gene expression profiles and mechanisms of toxicity. Toxicology letters 120, 359368 (2001).

21 Barrier, M. \& Mirkes, P. E. Proteomics in developmental toxicology. Reprod Toxicol 19, 291-304 (2005).

22 Mosmann, T. Rapid colorimetric assay for cellular growth and survival: application to proliferation and cytotoxicity assays. J Immunol Methods 65, 55-63 (1983).

23 Anderson, P. O., Knoben, J. E. \& Troutman, W. G. Handbook of clinical drug data. 1148 (2002). 
24 Bouwman, F. G. et al. The Physiologic Effects of Caloric Restriction Are Reflected in the in Vivo AdipocyteEnriched Proteome of Overweight/Obese Subjects. J Proteome Res 8, 5532-5540 (2009).

25 Dumont, D., Noben, J. P., Raus, J., Stinissen, P. \& Robben, J. Proteomic analysis of cerebrospinal fluid from multiple sclerosis patients. Proteomics 4, 2117-2124 (2004).

26 Hewitt, N. J. \& Hewitt, P. Phase I and II enzyme characterization of two sources of HepG2 cell lines. Xenobiotica 34, 243-256 (2004).

27 Bessems, J. G. \& Vermeulen, N. P. Paracetamol (acetaminophen)-induced toxicity: molecular and biochemical mechanisms, analogues and protective approaches. Crit Rev Toxicol 31, 55-138 (2001).

28 Zahno, A. et al. The role of CYP3A4 in amiodarone-associated toxicity on HepG2 cells. Biochem Pharmacol 81, 432-441 (2010).

29 Satoh, T. \& Hosokawa, M. Structure, function and regulation of carboxylesterases. Chem Biol Interact 162, 195211 (2006).

30 Talcott, R. E., Pond, S. M., Ketterman, A. \& Becker, C. E. Ethylesterases as indicators of liver damage. I. Studies on malathion carboxylesterases. Toxicol Appl Pharmacol 65, 69-74 (1982).

31 Raffalli-Mathieu, F., Glisovic, T., Ben-David, Y. \& Lang, M. A. Heterogeneous nuclear ribonucleoprotein A1 and regulation of the xenobiotic-inducible gene Cyp2a5. Mol Pharmacol 61, 795-799 (2002).

32 Jin, Y. \& Penning, T. M. Aldo-keto reductases and bioactivation/detoxication. Annu Rev Pharmacol Toxicol 47, 263-292 (2007).

33 Belin, M. W., Bouchard, C. S. \& Phillips, T. M. Update on topical cyclosporin A. Background, immunology, and pharmacology. Cornea 9, 184-195 (1990).

34 Akashi, M., Tanaka, A. \& Takikawa, H. Effect of cyclosporin A on the biliary excretion of cholephilic compounds in rats. Hepatol Res 34, 193-198 (2006).

35 Ryffel, B., Woerly, G., Rodriguez, C. \& Foxwell, B. M. Identification of the multidrug resistance-related membrane glycoprotein as an acceptor for cyclosporine. J Recept Res 11, 675-686 (1991).

36 Trauner, M. \& Boyer, J. L. Bile salt transporters: molecular characterization, function, and regulation. Physiol Rev 83, 633-671 (2003).

37 Alrefai, W. A. \& Gill, R. K. Bile acid transporters: structure, function, regulation and pathophysiological implications. Pharm Res 24, 1803-1823 (2007).

38 Lodish, H. F. \& Kong, N. Cyclosporin A inhibits an initial step in folding of transferrin within the endoplasmic reticulum. J Biol Chem 266, 14835-14838 (1991).

39 Russell, R. G. et al. Cyclosporin A. Mode of action and effects on bone and joint tissues. Scand J Rheumatol Suppl 95, 9-18 (1992).

40 Kockx, M. et al. Cyclosporin A decreases apolipoprotein E secretion from human macrophages via a protein phosphatase 2B-dependent and ATP-binding cassette transporter A1 (ABCA1)-independent pathway. J Biol Chem 284, 24144-24154 (2009).

41 Ryffel, B. et al. Cyclosporine--relationship of side effects to mode of action. Transplantation 46, 90S-96S (1988).

42 Bernardi, P. Mitochondrial transport of cations: channels, exchangers, and permeability transition. Physiol Rev 79, 1127-1155 (1999).

43 Landolfi, B., Curci, S., Debellis, L., Pozzan, T. \& Hofer, A. M. Ca2+ homeostasis in the agonist-sensitive internal store: functional interactions between mitochondria and the ER measured In situ in intact cells. $J$ Cell Biol 142, 1235-1243 (1998)

44 Arora, P. D., Silvestri, L., Ganss, B., Sodek, J. \& McCulloch, C. A. Mechanism of cyclosporin-induced inhibition of intracellular collagen degradation. J Biol Chem 276, 14100-14109 (2001)

45 Fomina, A. F., Fanger, C. M., Kozak, J. A. \& Cahalan, M. D. Single channel properties and regulated expression of $\mathrm{Ca}(2+)$ release-activated $\mathrm{Ca}(2+)$ (CRAC) channels in human T cells. J Cell Biol 150, 1435-1444 (2000).

46 Meldolesi, J. \& Pozzan, T. The endoplasmic reticulum Ca2+ store: a view from the lumen. Trends Biochem Sci 23, 10-14 (1998).

47 Bonilla, M., Nastase, K. K. \& Cunningham, K. W. Essential role of calcineurin in response to endoplasmic reticulum stress. Embo J 21, 2343-2353 (2002).

48 Crawford, J. M., Berken, C. A. \& Gollan, J. L. Role of the hepatocyte microtubular system in the excretion of bile salts and biliary lipid: implications for intracellular vesicular transport. J Lipid Res 29, 144-156 (1988). 
49 Roman, I. D., Monte, M. J., Gonzalez-Buitrago, J. M., Esteller, A. \& Jimenez, R. Inhibition of hepatocytary vesicular transport by cyclosporin A in the rat: relationship with cholestasis and hyperbilirubinemia. Hepatology 12, 83-91 (1990).

50 Le Goff, W. et al. Cyclosporin A traps ABCA1 at the plasma membrane and inhibits ABCA1-mediated lipid efflux to apolipoprotein A-I. Arterioscler Thromb Vasc Biol 24, 2155-2161 (2004).

51 Tsujita, M. et al. On the hepatic mechanism of HDL assembly by the ABCA1/apoA-I pathway. J Lipid Res 46, 154162 (2005).

52 Ito, J., Nagayasu, Y., Kato, K., Sato, R. \& Yokoyama, S. Apolipoprotein A-I induces translocation of cholesterol, phospholipid, and caveolin-1 to cytosol in rat astrocytes. J Biol Chem 277, 7929-7935 (2002).

53 Kheirollah, A., Ito, J., Nagayasu, Y., Lu, R. \& Yokoyama, S. Cyclosporin A inhibits apolipoprotein A-I-induced early events in cellular cholesterol homeostasis in rat astrocytes. Neuropharmacology 51, 693-700 (2006).

54 Moyer, K. \& Balistreri, W. Hepatobiliary disease in patients with cystic fibrosis. Curr Opin Gastroenterol 25, 272 278 (2009).

55 Childers, M., Eckel, G., Himmel, A. \& Caldwell, J. A new model of cystic fibrosis pathology: lack of transport of glutathione and its thiocyanate conjugates. Med Hypotheses 68, 101-112 (2007).

56 Pusl, T. \& Beuers, U. Intrahepatic cholestasis of pregnancy. Orphanet J Rare Dis 2, 26 (2007).

57 Yamamoto, Y. et al. Estrogen receptor alpha mediates 17alpha-ethynylestradiol causing hepatotoxicity. The Journal of biological chemistry 281, 16625-16631, (2006).

58 Kikkawa, R. et al. In vivo hepatotoxicity study of rats in comparison with in vitro hepatotoxicity screening system. J Toxicol Sci 31, 23-34 (2006).adipo

59 Yamamoto, T., Kikkawa, R., Yamada, H. \& Horii, I. Identification of oxidative stress-related proteins for predictive screening of hepatotoxicity using a proteomic approach. J Toxicol Sci 30, 213-227 (2005).

60 Yamamoto, T., Kikkawa, R., Yamada, H. \& Horii, I. Investigation of proteomic biomarkers in in vivo hepatotoxicity study of rat liver: toxicity differentiation in hepatotoxicants. J Toxicol Sci 31, 49-60 (2006). 


\section{CHAPTER 3}

\section{Screening for drug-induced hepatotoxicity in}

primary mouse hepatocytes using acetaminophen, amiodarone and cyclosporin $A$ as model compounds: an 'omics guided approach

Anke Van Summeren, Johan Renes, Daneida Lizarraga, Freek G. Bouwman, Jean-Paul Noben, Joost H. M. van Delft, Jos C. S. Kleinjans and Edwin C. M. Mariman

Omics : a journal of integrative biology 17, 71-83 (2013). 


\begin{abstract}
Drug-induced hepatotoxicity is a leading cause of attrition for candidate pharmaceuticals in development. New pre-clinical screening methods are crucial to predict drug toxicity prior to human studies. Of all in vitro hepatotoxicity models, primary human hepatocytes are considered as 'the gold standard'. However, their use is hindered by limited availability and interindividual variation. These barriers may be overcome by using primary mouse hepatocytes. We used difference gel electrophoresis (DIGE) to study large scale protein expression of primary mouse hepatocytes. These hepatocytes were exposed to three well defined hepatotoxicants: acetaminophen, amiodarone and cyclosporin A. Each hepatotoxicant induces a different hepatotoxic phenotype. Based on the DIGE results, the mRNA expression levels of deregulated proteins from cyclosporin A-treated cells were also analyzed. We were able to distinguish the cyclosporin A samples from the control, as well as the acetaminophen and amiodarone-treated samples. Cyclosporin A induced endoplasmic reticulum (ER) stress and altered the ER-Golgi transport. Moreover, liver carboxylesterase and bile salt sulfotransferase were differentially expressed. These proteins were associated with a protective adaptive response against cyclosporin A-induced cholestasis. The results of this study are comparable with effects seen in HepG2 cells. Therefore, we suggest both models can be used to analyze the cholestatic properties of cyclosporin A. Furthermore, this study showed a conserved response between primary mouse hepatocytes and HepG2 cells. These findings collectively lend support to use of 'omics strategies in preclinical toxicology, and might inform future efforts to better link preclinical and clinical research in rational drug development.
\end{abstract}




\section{INTRODUCTION}

The liver is responsible for detoxification and elimination of potentially harmful substances. Therefore, it is an important target organ for xenobiotic compounds. For that reason, hepatotoxicity is the most prominent adverse drug reaction leading to the failure of candidate drugs in preclinical or clinical trials. New screening methods, which can detect drug-induced liver injury at an early stage of the drug development, represent an important step towards rational drug development.

In xenobiotic metabolism and toxicity studies, primary hepatocytes are considered as the current standard of in vitro models ${ }^{1}$. The isolation of primary hepatocytes was introduced by Seglen ${ }^{2}$. He developed the two-step collagenase perfusion protocol to obtain viable rat hepatocytes. Primary rat and to a lesser extent human hepatocytes, are currently established in vitro systems. Previously it was shown that primary human hepatocytes have a similar expression pattern of the biotransformation genes as human liver tissue ${ }^{3}$. However, the use of primary human hepatocytes is hindered by the scarcity of suitable liver samples and by considerable inter-individual variation due to genetic, environmental and age differences of the donors ${ }^{4}$. Furthermore, the longer the culturing, both human as the alternative primary rat hepatocytes show a relatively rapid decline of their cytochrome P450 (CYP450) enzyme activities ${ }^{5}$. Based on their gene expression and CYP450 enzyme activity, primary mouse hepatocytes maintain their metabolic competence better than rat hepatocytes ${ }^{6}$. Moreover, transgenic mouse models are widely available, which may provide primary hepatocytes suitable for dedicated mechanistic investigations of liver toxicity. For instance hepatocytes from the DNA repair-deficient $\mathrm{Xpa}^{-/-} \mathrm{p53^{+/- }}$ mouse have been considered as a model for carcinogenicity screening ${ }^{7}$. As such, primary mouse hepatocytes seem a promising model for hepatic toxicity studies.

Beside primary hepatocytes, human cell lines as HepG2 cells are established models for toxicity studies. It has been shown that HepG2 cells are able to metabolize xenobiotic compounds leading to toxic effects, including genotoxicity, oxidative stress and mitochondrial dysfunction ${ }^{8-10}$. However, it is undeniable that these cells have lost some liver specific functions due to immortalization, in particular the phase I drug metabolizing enzymes ${ }^{5,11}$.

The 'omics-technologies provide powerful tools for expression profiling of biological events, and have the potential to improve current toxicity tests ${ }^{12}$. The conventional toxicity tests mostly rely on the examination of classical biological endpoints. It can take weeks, months, or even years before these traditional toxicological endpoints occur. Specific changes in protein and mRNA expression could occur within a few hours or days after exposure to chemical compounds.

Within the 'omics-field the whole genome gene expression analysis is still the driving technology. But it is acknowledged that the relative gene expression levels often only moderately correlate with the relative abundance of its protein product. This moderate correlation is due to the turnover differences of proteins and mRNA ${ }^{13}$. Moreover, post-translational modifica- 
tions and protein interactions are not detected by transcriptomics, in contrast to proteomics techniques. For example, with DIGE proteins are separated based on their $\mathrm{pl}$ and molecular weight, so different protein isoforms can be visualized.

Previously we have studied the proteome of HepG2 cells after incubation with three well defined hepatotoxicants: acetaminophen, amiodarone and cyclosporin A. They each represent a different class of hepatotoxicity ${ }^{14}$. Cyclosporin $A$ induced the most prominent effect on the proteome of the HepG2 cells. Furthermore, the protein expression profile of the cyclosporin-A treated cells could be distinguished from the protein expression profiles generated by the control acetaminophen- and the amiodarone-treated cells.

The use of primary hepatocytes is preferred in toxicological studies, because they maintain well their metabolizing capacities. For that reason we analyzed the proteome of primary mouse hepatocytes for its ability to discriminate between different types of drug-induced hepatotoxicity, and the level of conservation of response in comparison with a human in vitro model. In this study, we analyzed the protein expression in primary mouse hepatocytes after exposure to acetaminophen, amiodarone and cyclosporin $A$ and compared it with the protein expression in HepG2, obtained from our previous study ${ }^{14}$. To investigate the correlation between the protein and mRNA expression we compared the differentially expressed proteins induced by cyclosporin $\mathrm{A}$, with their corresponding mRNA expression.

\section{MATERIALS AND METHODS}

\section{Chemicals}

Dulbecco's Modified Eagle's medium, fetal calf serum, penicillin/streptomycin, Hanks' calcium- and magnesium-free buffer and insulin were obtained from Life Technologies Europe BV (Bleiswijk, The Netherlands). Glucagon, hydrocortisone (water soluble), collagenase type IV, dimethylsulfoxide (DMSO), trypan blue, 3-(4,5-dimethylthiazol-2-yl)-2,5-diphenyltetrazolium bromide, $\mathrm{NaCl}, \mathrm{NaHCO}_{3}, \mathrm{KCl}, \mathrm{KH}_{2} \mathrm{PO}_{4} ; \mathrm{MgSO}_{4}$, glucose; $\mathrm{CaCl}_{2}$, acetaminophen, amiodarone, cyclosporin A (BioChemika) and N,N-dimethylformamide (anhydrous, $99.8 \%$ ) were purchased from Sigma-Aldrich (Zwijndrecht, The Netherlands). Collagen Type I Rat Tail was obtained from BD BioSciences (Bedford, MA, USA), the Protein Assay Kit and non-fat dry milk powder (NFDM) was from Bio-Rad (Veenendaal, The Netherlands). All chemicals used for DIGE were purchased from GE Healthcare (Diegem, Belgium). The antibody against perilipin2/adipophilin (Plin2) used for Western blotting was purchased from Abcam (Cambridge, UK). The antibody against $\beta$-Actin and bile sulfotransferase were obtained from Santa Cruz Biotechnology (Heidelberg, Germany). The Horseradish peroxidase-conjugated secondary antibodies rabbit anti mouse and swine anti rabbit were obtained from DAKO (Enschede, The Netherlands). The chemiluminescent substrate (SuperSignal $\mathrm{CL}$ ) was purchased from Thermo Fisher Scientific (Etten-Leur, The Netherlands). The Trizol reagent and the RNeasy mini kit were from Qiagen Westburg (Leusden, The Netherlands). 


\section{Animals}

Permission for animal studies was obtained from the Animal Ethical Committee of the Maastricht University, The Netherlands (approval no. 2008-075). Adult male C57/B6 mice, weighing 20-25 g, were obtained from Charles River $\mathrm{GmbH}$, Sulzfeld, Germany. The animals were housed in macrolon cages with sawdust bedding at $22^{\circ} \mathrm{C}$ and $50-60 \%$ humidity. The light cycle was $12 \mathrm{~h}$ light/12 h dark. Food and tap water were available ad libitum.

\section{Isolation and culturing of primary mouse hepatocytes}

Hepatocytes were isolated by the two-step collagenase perfusion method according to Seglen ${ }^{2}$, with modifications as described before ${ }^{6}$. Cell suspensions with cell viability $\geq 80 \%$, determined by trypan blue exclusion, were brought into culture in a collagen-collagen sandwich as described before ${ }^{6}$. Prior to treatment, the primary mouse hepatocytes were allowed to recover for $40-42 \mathrm{~h}$ at $37^{\circ} \mathrm{C}$ in a humidified chamber with $95 \%$ / $5 \%$ air / CO2 in serum-free culture medium supplemented with insulin $(0.5 \mathrm{U} / \mathrm{ml})$, glucagon $(7 \mathrm{ng} / \mathrm{ml})$, hydrocortisone $(7.5 \mu \mathrm{g} / \mathrm{ml})$, and $2 \%$ penicillin/streptomycin $(5000 \mathrm{U} / \mathrm{ml}$ penicillin and $5000 \mathrm{Im} / \mathrm{ml}$ streptomycin). Culture medium was refreshed every $24 \mathrm{~h}$. After the recovery period, the culture medium was replaced by the culture medium containing one of the selected compounds or $0.5 \%$ DMSO, as a vehicle control. For each compound the $\mathrm{IC}_{20}$ concentration was determined by the MTT reduction method ${ }^{15}$. Based on the $\mathrm{IC}_{20}$ values, the hepatocytes were exposed as follows: $1 \mathrm{mM}$ acetaminophen, $1 \mu \mathrm{M}$ amiodarone and $10 \mu \mathrm{M}$ cyclosporin A or $0.5 \%$ DMSO as a vehicle control for 48 hours. For proteome analysis, five independent biological experiments, each with cells from a different animal, were performed. For transcriptomics, three new independent biological experiments were conducted.

\section{Protein extraction}

The cells were washed twice with PBS. Before the protein extraction, the collagen layers were removed from the hepatocytes to prevent its interference with the proteome analysis. Intact cells were isolated from the collagen layers after 10 min incubation with collagenase buffer. The collagenase buffer contains $1150 \mathrm{CDU} / 100 \mathrm{ml}$ collagenase $(\mathrm{CDU}=$ collagen digestion units) dissolved in a krebs buffer $(118 \mathrm{mM} \mathrm{NaCl} ; 25 \mathrm{mM} \mathrm{NaHCO}$; $4.8 \mathrm{mM} \mathrm{KCl} ; 1.2 \mathrm{mM}$ $\mathrm{KH}_{2} \mathrm{PO}_{4} ; 1.2 \mathrm{mM} \mathrm{MgSO}_{4} ; 11 \mathrm{mM}$ glucose; $1.5 \mathrm{mM} \mathrm{CaCl}_{2}$ ). The suspension of detached cells was washed and further diluted till $50 \mathrm{ml}$ with PBS, and centrifuged for $5 \mathrm{~min}$ at $65 \mathrm{~g}$. To wash the cells thoroughly, the washing step was repeated. Afterwards the supernatant was removed and the cell pellet was dissolved in a DIGE labeling buffer containing $7 \mathrm{M}$ urea, $2 \mathrm{M}$ thiourea, $4 \%(\mathrm{w} / \mathrm{v})$ CHAPS and $30 \mathrm{mM}$ Tris- $\mathrm{HCl}$. This mixture was subjected to three cycles of freeze thawing with liquid nitrogen, vortexed thoroughly and centrifuged at $20000 \mathrm{~g}$ for $30 \mathrm{~min}$ at $10^{\circ} \mathrm{C}$. Supernatant was collected, aliquoted and stored at $-80^{\circ} \mathrm{C}$ until further analysis. Protein concentrations were determined with the Protein Assay Kit from Bio-Rad (Veenendaal, The Netherlands). 
The total RNA from hepatocytes treated with cyclosporin A was isolated, using Trizol reagent with the RNeasy kit according to the manufacturer's protocol. Total cellular RNA levels were measured with a spectrophotometer and the quality of each RNA preparation was determined with a bio-analyzer (Agilent Technologies, The Netherlands). Extracted RNA was stored at $-80^{\circ} \mathrm{C}$.

\section{DIGE}

The protein labeling and the DIGE were performed as described before ${ }^{14}$. A one-way ANOVA analysis $(P \leq 0.05)$ was used to select the significant differential spots between the experimental groups. The EDA module of the DeCyder software was also used to perform a hierarchical clustering analysis and a principle component analysis (PCA). The differentially expressed proteins $(\mathrm{P} \leq 0.05)$ were excised and identified by a matrix assisted laser desorption ionization time-of-flight (MALDI-TOF/TOF) mass spectrometer (4800 MALDI TOF/TOF analyzer, Applied Biosystems) ${ }^{16}$. Protein spots that could not be identified via MALDI-TOF/TOF were further analyzed by nano liquid chromatography tandem mass spectrometry (nLC-MS/MS) on an LCQ Classic (ThermoFinnigan) as described ${ }^{17}$.

\section{Western blotting}

Samples with equal amount of protein ( $30 \mu \mathrm{g} /$ lane) were separated by SDS-PAGE on $4-12 \%$ Bis-Tris Criterion gels (Bio-Rad, Hercules, CA), at $150 \mathrm{~V}$ and transferred to a $0.45 \mathrm{~mm}$ nitrocellulose membrane for $90 \mathrm{~min}$ at $100 \mathrm{~V}$. After Ponceau S staining and destaining, membranes were blocked in $5 \%$ NFDM in Tris-buffered saline containing $0.1 \%$ Tween 20 (TBST) for $1 \mathrm{~h}$. Thereafter, the blots were incubated with the primary antibodies against Adipophilin (1:500 dilution), in $5 \%$ NFDM-TBST overnight at $4^{\circ} \mathrm{C}$ on a shaker. The blot was washed three times for $10 \mathrm{~min}$ in TBST and then incubated for $1 \mathrm{~h}$ with a 1:10 000 dilution of the horseradish peroxidase-conjugated secondary antibody in $5 \%$ NFDM-TBST. The blots were washed three times for $10 \mathrm{~min}$ in TBST. To detect bile salt sulfotransferase (Sult2A1) the same procedure was repeated, except for blocking (5\% BSA) and the dilution of the first antibody (1:200). A CCD camera (XRS-system, Bio-Rad) was used to detect immunoreactive bands using chemiluminescent substrate (SuperSignal $\mathrm{CL}$ ). The quantification was performed with the program Quantity One version 4.6.5 (Bio-Rad). $\beta$-Actin was used as reference for the amount of protein loaded.

\section{Microarray analysis}

The targets were prepared according to the Affymetrix protocol. Data analysis was performed as described earlier ${ }^{18}$. Only the genes of the differentially expressed proteins were selected and retrieved from the transcriptome data. Fold change calculations and student T-tests were performed in Microsoft Excel. Differentially expressed 
genes with a $p$-value $\leq 0.05$ and a $\mid$ fold change $\mid \geq 1.5$ were considered as significant.

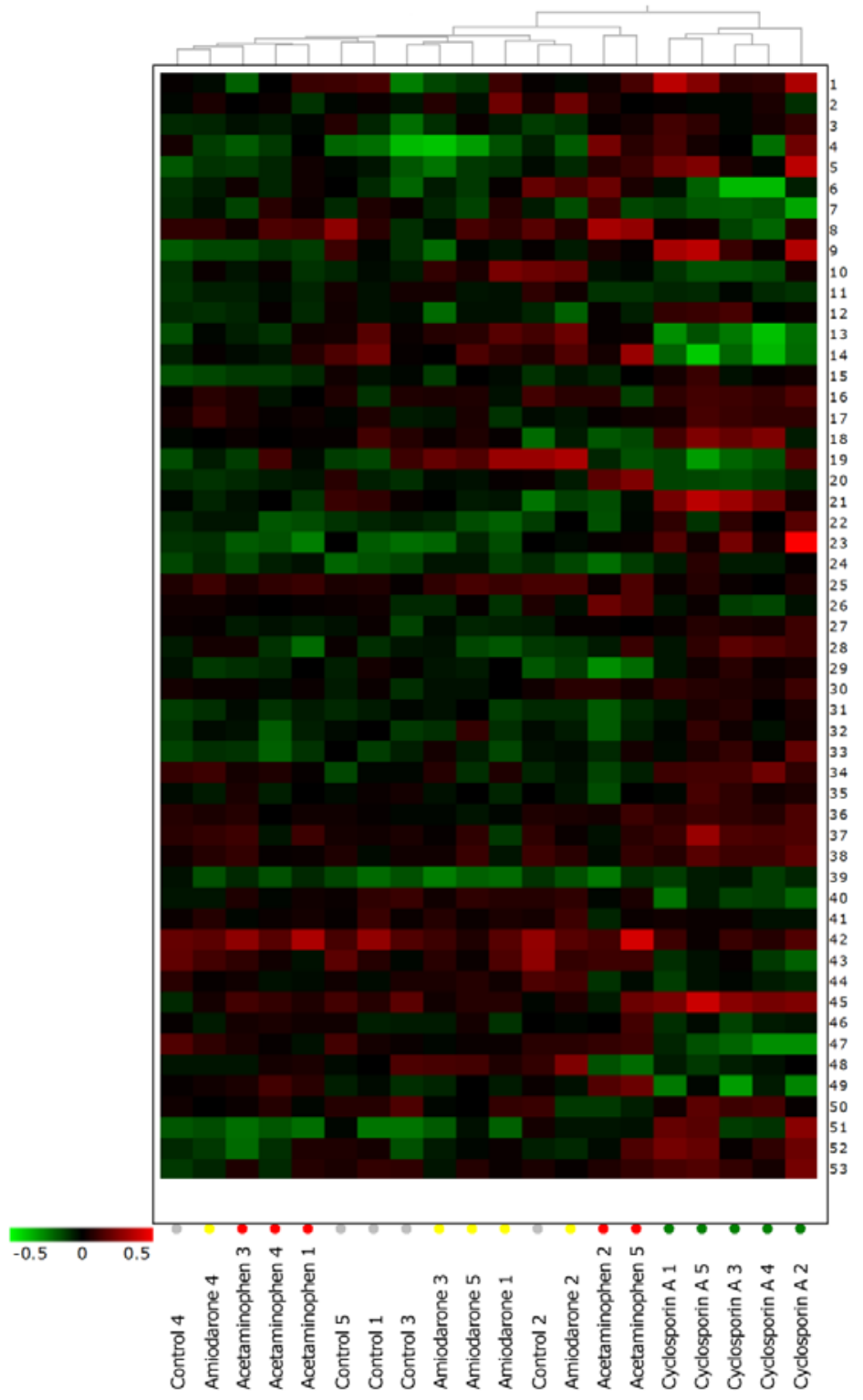

Figure 3.1: Hierarchical cluster analysis of the experimental groups (control, acetaminophen, amiodarone and cyclosporin A). The clustering is based on the log standard abundance of the significant differential spots (P-value $\leq 0.05)$ with a hierarchical clustering algorithm in the EDA module of the Decyder software. 


\section{RESULTS}

\section{Effect of the hepatotoxicants on the proteome of primary mouse hepatocytes}

The cellular proteins of the treated primary mouse hepatocytes were analyzed using DIGE. In total 1866 protein spots could be matched with all the images. With a one-way ANOVA analysis $(P \leq 0.05) 53$ spots were detected as significantly differential. Based on the Tukey's multiple comparison test, significantly differential protein expression was observed with cyclosporin $\mathrm{A}$, but not with acetaminophen and amiodarone. The experimental groups (control, acetaminophen, amiodarone and cyclosporin A) were clustered based on the log standard abundance of the 53 differential spots with a hierarchical clustering algorithm. As shown in Figure 3.1, the spot maps of cyclosporin A are distinguished mostly from other spot maps. Differences were found between the control, acetaminophen and amiodarone. Although they were rather small causing them to cluster together. A PCA was performed on the 53 differential spots (Figure 3.2). The cyclosporin A treated hepatocytes were discriminated by PC1 accounting for a variance of $44.3 \%$.

\section{Protein identification from the differential spots}

The differential spots were included in a pick list and excised from a preparative gel. Protein identification was performed by in-gel digestion followed by MALDI-TOF/TOF or nLC MS/MS. Out of the 53 spots, the proteins of 43 spots were identified (Table 3.1). Figure 3.3 shows the 2-DE map made from the master gel with the 43 identified differential spots indicated with a number which corresponds to the numbers presented in Table 3.1.

For spot numbers 1, 11, 14 and 17 multiple proteins for one spot were identified with $\mathrm{nLC}$ $\mathrm{MS} / \mathrm{MS}$, both protein identifications delivering the same number of peptides. Consequently, for these spots it is not possible to conclude which protein is responsible for the significant change of the fold change. Nine spots appeared isoforms from four proteins due to posttranslational modifications or processing of the protein. 


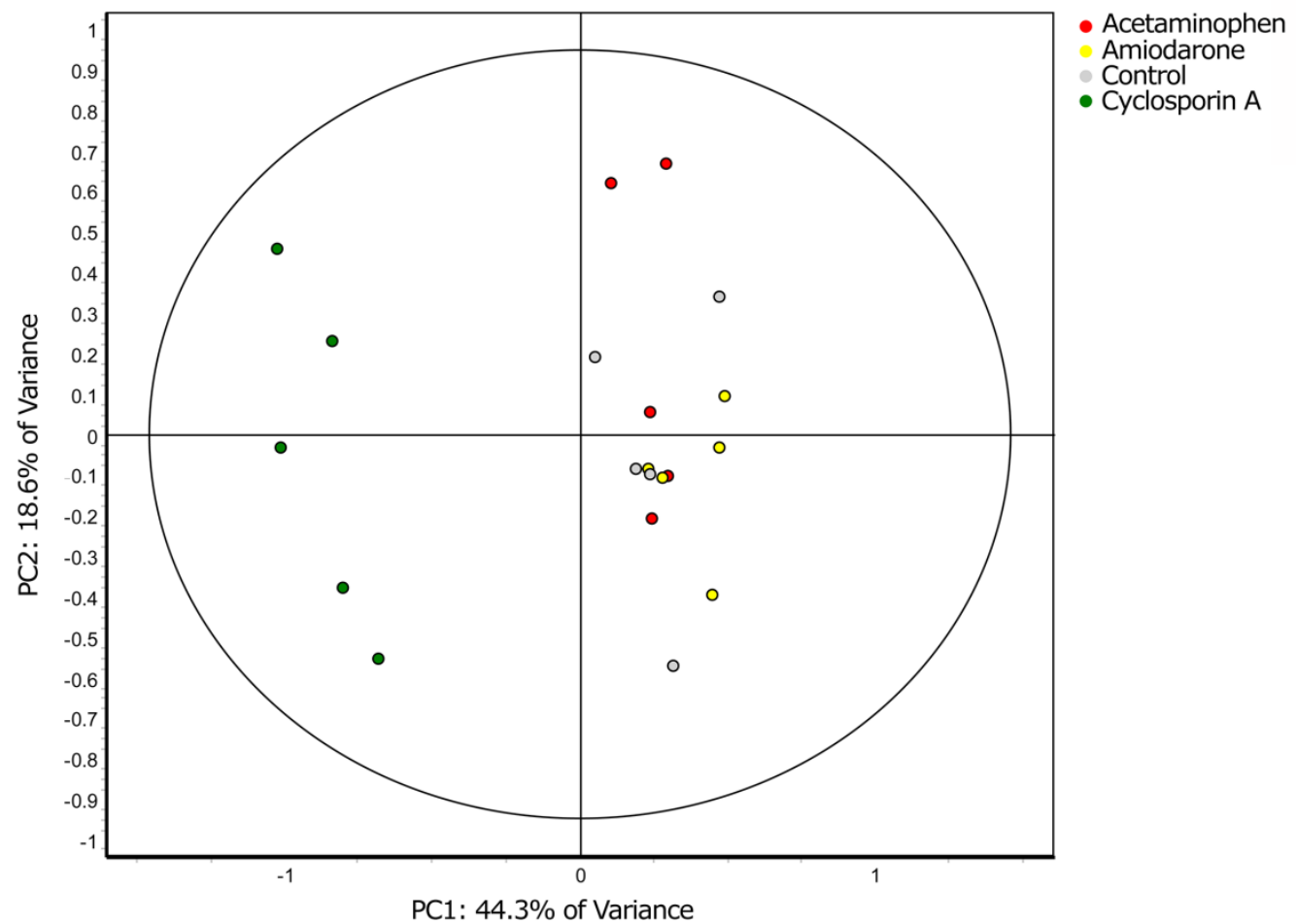

Figure 3.2: PCA analysis of the experimental groups (control, acetaminophen, amiodarone and cyclosporin A), based on the significant differential spots (one-way ANOVA P-value $\leq 0.05$ ), performed with the EDA module of the Decyder software. 
Table 3.1: Protein identification of differentially expressed proteins in primary mouse hepatocytes after exposure to acetaminophen, amiodarone or cyclosporin A and the mRNA expression of the differentially expressed proteins after exposure to cyclosporin A.

\begin{tabular}{|c|c|c|c|c|c|c|c|c|c|c|}
\hline \multirow[t]{3}{*}{ No. } & \multirow{3}{*}{$\begin{array}{c}\text { Uniprot } \\
\text { Accession } \\
\text { No. }\end{array}$} & \multirow[t]{3}{*}{ Protein description } & \multicolumn{4}{|c|}{ Protein expression } & \multirow[t]{3}{*}{ HepG2 } & \multirow{3}{*}{$\begin{array}{l}\text { Gene } \\
\text { name }\end{array}$} & \multicolumn{2}{|c|}{ mRNA expression } \\
\hline & & & \multirow{2}{*}{$\begin{array}{l}\text { P-value } \\
\text { (one-way } \\
\text { ANOVA) }\end{array}$} & \multicolumn{3}{|c|}{ Fold change } & & & \multirow{2}{*}{$\begin{array}{l}\text { P-value } \\
\text { (T-test) }\end{array}$} & \multirow{2}{*}{ 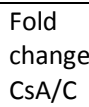 } \\
\hline & & & & $\mathrm{CsA} / \mathrm{C}$ & $\mathrm{Ac} / \mathrm{C}$ & $\mathrm{Am} / \mathrm{C}$ & & & & \\
\hline \multicolumn{11}{|c|}{ Metabolic process } \\
\hline 1 & P17182 & Alpha-enolase & 0.000117 & $-2.25^{*}$ & 1 & -1 & yes & Eno1 & $\mathrm{N} / \mathrm{A}$ & $\mathrm{N} / \mathrm{A}$ \\
\hline 1 & A2RSX9 & Arfaptin-1 protein & 0.00012 & $-2.25^{*}$ & 1 & -1 & & Arfip1 & 0.790 & -1.02 \\
\hline 2 & P11725 & Ornithine carbamoyltransferase, mitochondrial & 0.00385 & $2.12^{*}$ & -1.02 & -1.03 & & Otc & 0.0336 & $-6.53^{\ddagger}$ \\
\hline 5 & P09103 & Protein disulfide-isomerase & 0.01132 & $1.89^{*}$ & -1.08 & -1.16 & & P4hb & 0.00208 & $1.64^{\ddagger}$ \\
\hline 11 & Q9D1T5 & Proline-rich protein 15 & 0.00109 & $1.63^{*}$ & 1.07 & -1.04 & & Prr15 & 0.711 & -1.03 \\
\hline 11 & Q80ZP8 & $\begin{array}{l}\text { Mesencephalic astrocyte-derived neurotrophic } \\
\text { factor }\end{array}$ & 0.00109 & $1.63^{*}$ & 1.07 & -1.04 & & Manf & 0.00618 & $3.55^{\ddagger}$ \\
\hline 13 & P40936 & Indolethylamine $\mathrm{N}$-methyltransferase & 0.019 & $1.54^{*}$ & 1.06 & -1.03 & & Inmt & 0.182 & -1.26 \\
\hline 14 & P27773 & Protein disulfide-isomerase $\mathrm{A} 3$ & 0.022 & $-1.46^{*}$ & 1.05 & -1.07 & yes & Pdia3 & 0.0730 & 1.46 \\
\hline 15 & P16858 & $\begin{array}{l}\text { Glyceraldehyde-3-phosphate dehydrogenase } \\
\text { isoform } 1\end{array}$ & 0.0426 & -1.46 & 1.18 & 1.02 & & Gapdh & N/A & N/A \\
\hline 16 & P52843 & Bile salt sulfotransferase & 0.0229 & 1.45 & 1.06 & -1.05 & & Sult2a1 & $\mathrm{N} / \mathrm{A}$ & N/A \\
\hline 17 & Q60854 & Serpin B6 & 0.0288 & 1.43 & -1.16 & -1.01 & & $\begin{array}{l}\text { SER- } \\
\text { PINB6 }\end{array}$ & 0.0311 & $1.68^{\ddagger}$ \\
\hline 18 & Q8BGT5 & Alanine aminotransferase 2 & 0.0418 & -1.41 & 1.19 & -1.11 & & Gpt2 & 0.000636 & $-1.89^{\ddagger}$ \\
\hline 19 & P08003 & Protein disulfide-isomerase A4 & 0.00991 & $1.4^{*}$ & 1.17 & 1.05 & yes & Pdia4 & 0.0248 & $1.84^{\ddagger}$ \\
\hline 25 & P60843 & Eukaryotic initiation factor $4 \mathrm{~A}-\mathrm{I}$ & 0.045 & -1.26 & 1.24 & -1.04 & yes & Eif4a1 & N/A & $\mathrm{N} / \mathrm{A}$ \\
\hline 26 & Q922R8 & Protein disulfide-isomerase A6 & 0.046 & 1.25 & 1.01 & -1.09 & yes & Pdia6 & 0.0118 & $2.30^{\ddagger}$ \\
\hline 32 & P70195 & Proteasome subunit beta type- 7 & 0.0302 & $1.22^{*}$ & 1.09 & 1.04 & & Psmb7 & 0.0598 & 1.12 \\
\hline 33 & P54775 & $26 S$ protease regulatory subunit $6 B$ & 0.0427 & 1.21 & -1.06 & -1.03 & & Psmc4 & 0.299 & -1.09 \\
\hline 34 & P16460 & Argininosuccinate synthase & 0.025 & 1.21 & -1.1 & 1.02 & & ass1 & $\mathrm{N} / \mathrm{A}$ & $\mathrm{N} / \mathrm{A}$ \\
\hline 42 & P00375 & Dihydrofolate reductase & 0.0386 & -1.1 & -1.1 & 1.06 & & Dhfr & 0.0430 & $-1.68^{\ddagger}$ \\
\hline 43 & Q8C196 & Carbamoyl-phosphate synthase & 0.0471 & -1.02 & -1.02 & 1.3 & & Cps1 & $\mathrm{N} / \mathrm{A}$ & N/A \\
\hline \multicolumn{11}{|c|}{ Cellular Response } \\
\hline 20 & 070400 & PDZ and LIM domain protein 1 & 0.0195 & 1.39 & -1.04 & 1 & & Pdlim1 & 0.0479 & $1.54^{\ddagger}$ \\
\hline 21 & P60710 & Actin, cytoplasmic 1 & 0.04 & -1.37 & -1.19 & 1.48 & yes & Actb & 0.0276 & -1.12 \\
\hline
\end{tabular}




\begin{tabular}{|c|c|c|c|c|c|c|c|c|c|c|}
\hline \multirow[t]{3}{*}{ No. } & \multirow{3}{*}{$\begin{array}{c}\text { Uniprot } \\
\text { Accession } \\
\text { No. }\end{array}$} & \multirow[t]{3}{*}{ Protein description } & \multicolumn{4}{|c|}{ Protein expression } & \multirow[t]{3}{*}{ HepG2 } & \multirow{3}{*}{$\begin{array}{l}\text { Gene } \\
\text { name }\end{array}$} & \multicolumn{2}{|c|}{ mRNA expression } \\
\hline & & & \multirow{2}{*}{$\begin{array}{l}\text { P-value } \\
\text { (one-way } \\
\text { ANOVA) }\end{array}$} & \multicolumn{3}{|c|}{ Fold change } & & & P-value & Fold \\
\hline & & & & $\mathrm{CsA} / \mathrm{C}$ & $\mathrm{Ac} / \mathrm{C}$ & $\mathrm{Am} / \mathrm{C}$ & & & & $\mathrm{CsA} / \mathrm{C}$ \\
\hline 22 & P02535 & Keratin, type I cytoskeletal 10 & 0.013 & $1.35^{*}$ & 1.07 & 1.14 & \multirow{8}{*}{$\begin{array}{l}\text { yes } \\
\text { yes }\end{array}$} & Krt10 & 0.0313 & $1.61^{\ddagger}$ \\
\hline 23 & P11679 & Keratin, type II cytoskeletal 8 & 0.00909 & -1.28 & -1.1 & 1.31 & & Krt8 & 0.101 & -1.34 \\
\hline 32 & Q99P30 & $\begin{array}{l}\text { Isoform } 1 \text { of Peroxisomal coenzyme A diphos- } \\
\text { phatase NUDT7 }\end{array}$ & 0.0302 & $1.22^{*}$ & 1.09 & 1.04 & & Nudt7 & 0.120 & -1.38 \\
\hline 37 & P67778 & Prohibitin & 0.0449 & 1.16 & -1.21 & -1.06 & & phb & 0.0319 & -1.28 \\
\hline 38 & D3Z5W7 & Glutathione S-transferase, theta 1 & 0.013 & 1.16 & 1.04 & -1.06 & & Gstt1 & 0.0219 & $-3.28^{\ddagger}$ \\
\hline 36 & Q99LC5 & $\begin{array}{l}\text { Electron transfer flavoprotein subunit alpha, } \\
\text { mitochondrial }\end{array}$ & 0.0351 & -1.18 & 1.18 & -1.11 & & Etfa & 0.0135 & -1.42 \\
\hline 40 & P56395 & Cytochrome b5 & 0.00698 & -1.13 & 1.16 & -1.04 & & Cyb5a & 0.0367 & -1.34 \\
\hline 41 & P35700 & Peroxiredoxin-1 & 0.0373 & 1.11 & 1.01 & -1.17 & & Prdx1 & 0.600 & 1.03 \\
\hline \multicolumn{11}{|c|}{ Transport } \\
\hline 1 & P43883 & Perilipin2/adipophilin & 0.00012 & $-2.25^{*}$ & 1 & -1 & & Plin2 & 0.177 & -1.16 \\
\hline 3 & P43883 & Perilipin2/adipophilin & $6.19 \mathrm{E}-05$ & $-2.01^{*}$ & -1.17 & 1.14 & & Plin2 & 0.177 & -1.16 \\
\hline 4 & P24369 & Peptidyl-prolyl cis-trans isomerase B & 0.000011 & $-1.9^{*}$ & -1.08 & -1.12 & & Ppib & 0.0178 & 1.19 \\
\hline 8 & P24369 & Peptidyl-prolyl cis-trans isomerase B & 0.00298 & $-1.68^{*}$ & -1.21 & -1.14 & & Ppib & 0.0178 & 1.19 \\
\hline 6 & P07724 & Serum albumin precursor & 0.00147 & $1.86^{*}$ & 1.22 & -1.11 & & Alb & 0.0714 & -1.07 \\
\hline 12 & P07724 & Serum albumin & 0.0363 & 1.57 & 1.46 & -1.21 & yes & Alb & 0.0714 & -1.07 \\
\hline 9 & Q921I1 & Serotransferrin precursor & 0.0267 & 1.65 & 1.01 & -1.11 & yes & Tf & 0.0765 & -1.17 \\
\hline 24 & Q9D6F9 & Tubulin beta- 4 chain & 0.00229 & 1.28 & -1.01 & -1.18 & & Tubb4 & 0.908 & 1.03 \\
\hline 28 & Q60930 & $\begin{array}{l}\text { Voltage-dependent anion-selective channel } \\
\text { protein } 2\end{array}$ & 0.00886 & $1.24^{*}$ & 1.02 & 1.01 & & Vdac2 & 0.498 & 1.04 \\
\hline 29 & Q99J08 & SEC14-like protein 2 & 0.031 & 1.23 & 1.05 & -1.01 & & $\operatorname{Sec} 1412$ & 0.0190 & $-2.30^{\ddagger}$ \\
\hline \multicolumn{11}{|c|}{ Response to stimulus } \\
\hline 7 & P20029 & $78 \mathrm{kDa}$ glucose-regulated protein & 0.000763 & $1.69^{*}$ & -1.18 & -1.17 & yes & Hspa5 & 0.00323 & $2.11^{\ddagger}$ \\
\hline 10 & P20029 & $78 \mathrm{kDa}$ glucose-regulated protein & 0.045 & $1.64^{*}$ & -1.06 & -1.01 & yes & Hspa5 & 0.0032 & $2.11^{\ddagger}$ \\
\hline 17 & P20029 & $78 \mathrm{kDa}$ glucose-regulated protein & 0.0288 & 1.43 & -1.16 & -1.01 & yes & Hspa5 & 0.00323 & $2.11^{\ddagger}$ \\
\hline 14 & Q8VCC2 & Liver carboxylesterase 1 & 0.022 & $-1.46^{*}$ & 1.05 & -1.07 & yes & Ces1 & 0.0396 & $-6.27^{\ddagger}$ \\
\hline 27 & Q99KB8 & $\begin{array}{l}\text { Hydroxyacylglutathione hydrolase, mitochon- } \\
\text { drial }\end{array}$ & 0.00864 & $1.24^{*}$ & -1.04 & 1.01 & & Hagh & 0.00437 & 1.40 \\
\hline
\end{tabular}




\begin{tabular}{|c|c|c|c|c|c|c|c|c|c|c|}
\hline \multirow[t]{3}{*}{ No. } & \multirow{3}{*}{$\begin{array}{c}\text { Uniprot } \\
\text { Accession } \\
\text { No. }\end{array}$} & \multirow[t]{3}{*}{ Protein description } & \multicolumn{4}{|c|}{ Protein expression } & \multirow[t]{3}{*}{ HepG2 } & \multirow{3}{*}{$\begin{array}{l}\text { Gene } \\
\text { name }\end{array}$} & \multicolumn{2}{|c|}{ mRNA expression } \\
\hline & & & \multirow{2}{*}{$\begin{array}{l}\text { P-value } \\
\text { (one-way } \\
\text { ANOVA) }\end{array}$} & \multicolumn{3}{|c|}{ Fold change } & & & P-value & \\
\hline & & & & $\mathrm{CsA} / \mathrm{C}$ & $\mathrm{Ac} / \mathrm{C}$ & $\mathrm{Am} / \mathrm{C}$ & & & & $\mathrm{CsA} / \mathrm{C}$ \\
\hline 30 & P30115 & Glutathione S-transferase Yc & 0.037 & 1.23 & -1.01 & 1.01 & & Gsta3 & 0.0434 & $-1.85^{\ddagger}$ \\
\hline 31 & Q63836 & Selenium-binding protein 2 & 0.038 & -1.22 & -1.22 & -1.06 & & $\begin{array}{l}\text { Selenb } \\
\text { p2 }\end{array}$ & N/A & N/A \\
\hline 35 & P14602 & Isoform A of Heat shock protein beta-1 & 0.043 & 1.19 & -1.12 & 1.06 & & Hspb1 & $\# N / A$ & N/A \\
\hline 39 & Q9WVLO & Maleylacetoacetate isomerase & 0.0252 & 1.15 & -1.07 & -1.1 & & Gstz1 & 0.0574 & -2.10 \\
\hline
\end{tabular}

${ }^{1}$ P-value from one way ANOVA statistical test between the four groups with each five biological replicates.

${ }^{2}$ The difference in the standardized abundance of the proteins is expressed as the fold change between the control (C) and the treated groups ( $T$ ). The fold change is calculated by taking the means of standardized volume values for the protein spot in the corresponding groups $(C=\operatorname{control}, \mathrm{Cs} A=\mathrm{cyclosporin} \mathrm{A}, \mathrm{Ac}=\mathrm{acetaminophen}, \mathrm{Am}=\mathrm{amioda}-$ rone), values are calculated as $\mathrm{T} / \mathrm{C}$ and displayed in the range of +1 to $+\infty$ for increases in expression and calculated as $-\mathrm{C} / \mathrm{T}$ and displayed in the range of $-\infty$ to -1 for decreased expression.

* Indicates significant fold changes $(P \leq 0.05)$ between the control and the treated group, calculated with a multiple comparison test.

${ }^{\ddagger}$ Indicates significant fold changes $(P \leq 0.05 ;|F C| \geq 1.5)$ between the control and the cyclosporin A treated group, calculated with a student $T$-test. 
$\mathrm{pH} 3$

$\sim 200 \mathrm{kDa}$

pH 11

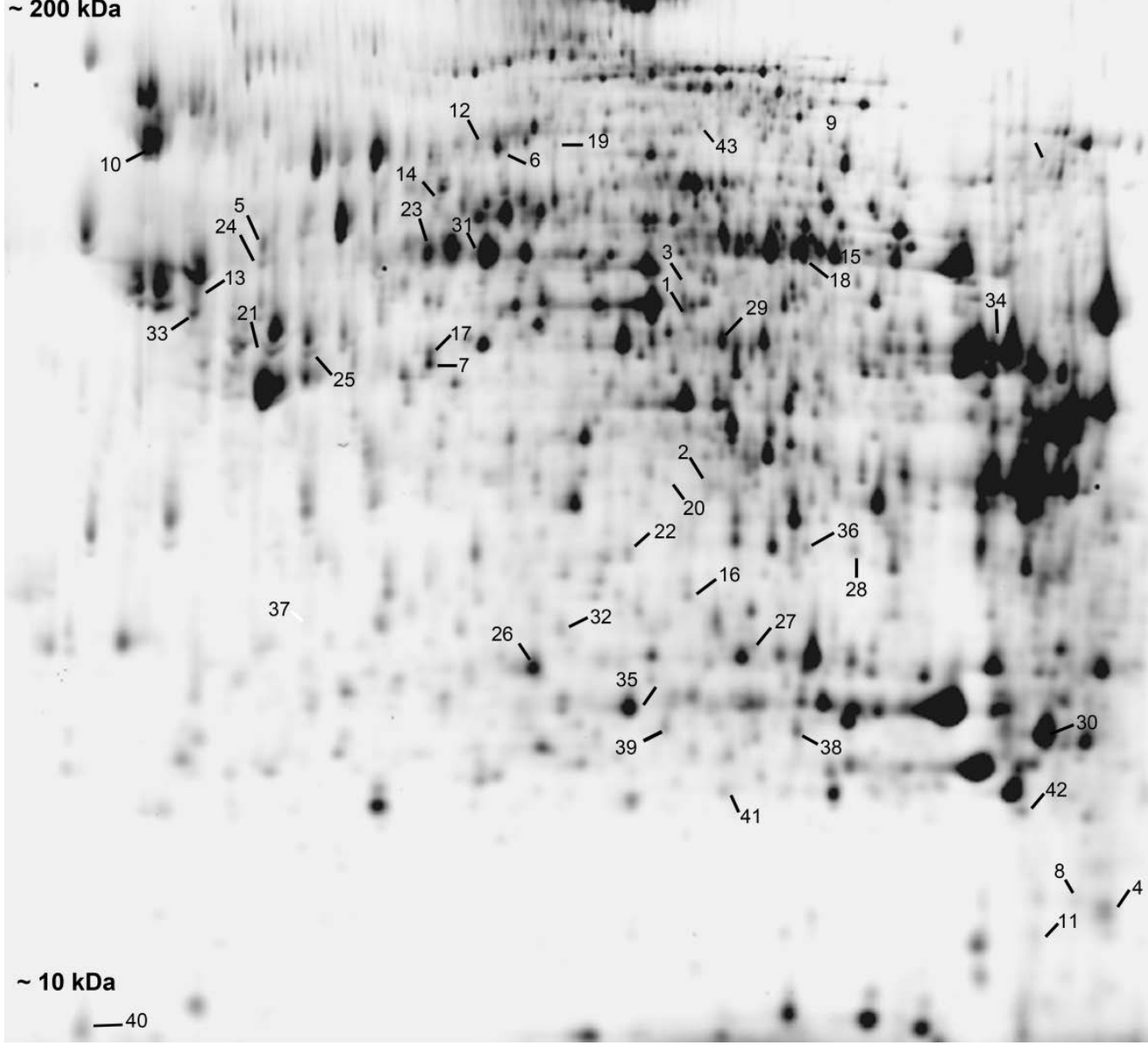

Figure 3.3: Proteome map of the differentially expressed proteins (One-way ANOVA P-value $\leq 0.05$ ), in primary mouse hepatocytes exposed to the hepatotoxicants Acetaminophen, Amiodarone or cyclosporin A.. All the identified spots are indicated with a number which corresponds to the numbers used in Table 3.1.

The functional properties of the identified proteins were obtained by the Panther classification system (http://www.pantherdb.org). The majority of the differential proteins are involved in transport, metabolic and cellular processes (Figure 3.4).

To confirm our findings obtained with the DIGE analysis, western blotting was performed on two significantly changed proteins, Plin2 and Sult2A1. Because of their role in the cholesterol metabolism these proteins are probably important for the mechanism behind cyclosporin Ainduced cholestasis. 


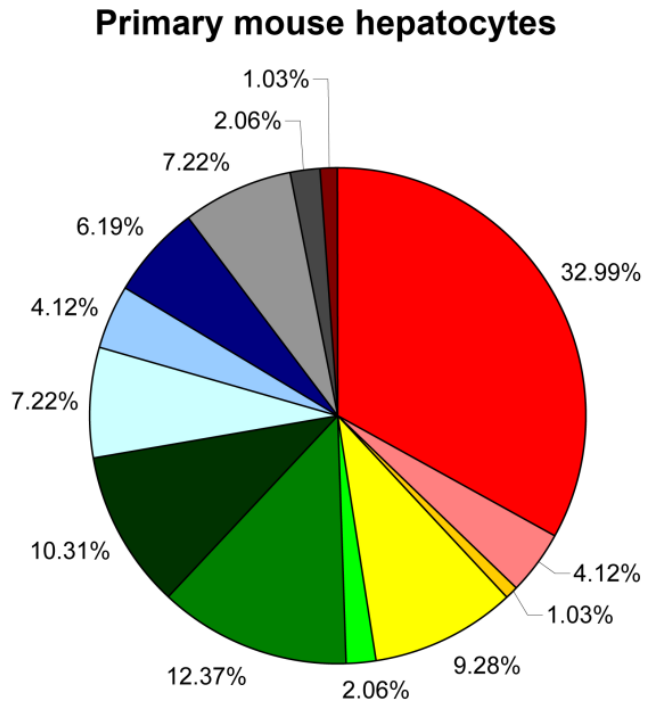

$\square$ metabolic process

$\square$ cell adhesion

$\square$ cell communication

- transport

$\square$ system process

response to stimulus

$\square$ generation of precursor metabolites and energy

\section{HepG2}

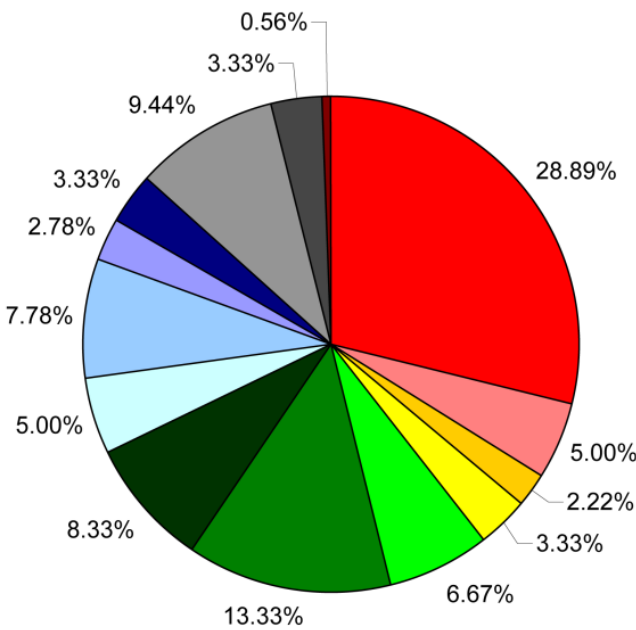

$\square$ cell cycle

$\square$ immune system process

$\square$ cellular process

$\square$ cellular component organization

$\square$ reproduction

$\square$ developmental process

unkown

Figure 3.4: Classification of the differential expressed proteins in HepG2 and primary mouse hepatocytes after exposure to cyclosporin A with the Panther classification system (http://www.pantherdb.org).

The DIGE analysis showed a decreased expression of Plin2 after cyclosporin A treatment. This was confirmed by western blotting, which showed a significantly decreased expression of this protein after cyclosporin A treatment with a P-value of 0.037 (Figure 3.5A). An insignificant decrease of this protein was found after treatment with the other hepatotoxicants. The DIGE analysis revealed an increased expression of Sult2A1 upon cyclosporin A treatment. The western blot showed a significantly increased expression for all three drugs with P-values of $0.0016,0.030,0.043$ for acetaminophen, amiodarone and cyclosporin A, respectively (Figure 3.5B).

\section{Correlation of the differentially expressed proteins with RNA expression}

The mRNA levels of the differentially expressed proteins from cyclosporin A-treated cells were retrieved from the micro-array data. We focused on the cyclosporin A treated cells, since all differentially expressed proteins were assigned to cyclosporin A. For the following significant- 
ly up-regulated proteins a corresponding up-regulation of their mRNA was found: protein disulfide-isomerase, protein disulfide-isomerase $A 6$, protein disulfide-isomerase $A 4$, keratin type I cytoskeletal 10, $78 \mathrm{kDa}$ glucose-regulated protein and mesencephalic astrocyte-derived neurotrophic factor. Liver carboxylesterase 1 (Ces1) shows a significant decrease on the proteome and transcriptome induced by cyclosporin A. Furthermore, cyclosporin A also downregulated protein expression of alanine aminotransferase 2 (Gpt2), however not significant according to the multiple comparison test. On the other hand Gpt2 was significantly downregulated on transcriptome. These data suggest that cellular changes due to 48 hours of cyclosporin A treatment can be the consequence of transcriptional adaptations. However, divergences between protein and RNA expression were also observed. For instance, the protein expression of ornithine carbamoyltransferase was found up-regulated with a fold change of 2.12 , while the RNA expression of the same protein was found highly down-regulated with a fold change of -6.53. Differences in protein and RNA expression, point out altered gene expression regulation at the translational or posttranslational level. No clear indication for posttranslational regulation was obtained, since the detected isoforms from Plin2 and $78 \mathrm{kDa}$ glucose-regulated protein changed all in the same direction.

A

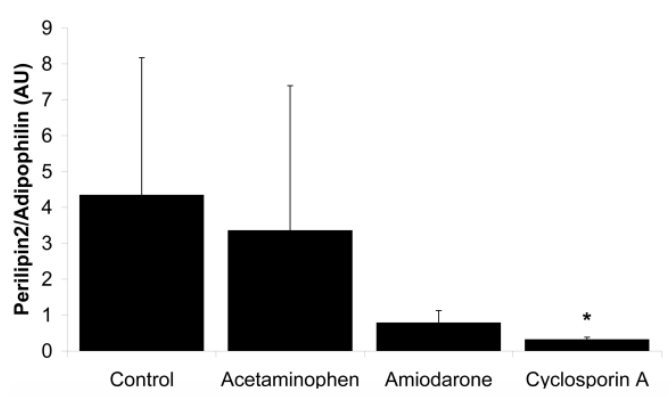

B

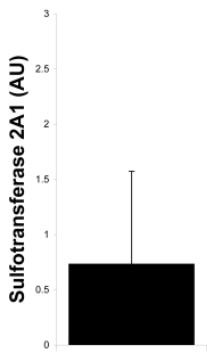

Control
Sulfotransferase 2A1

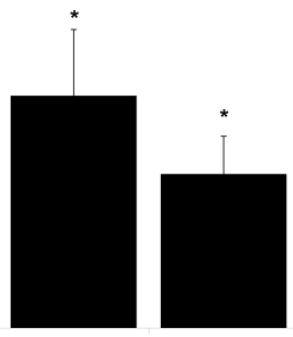

Acetaminophen Amiodarone

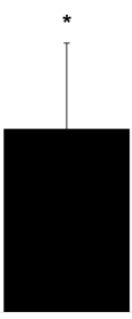

Cyclosporin A

Figure 3.5: Expression differences by western blotting of $(A)$ perilipin2/adipophilin and (B) bile salt sulfotransferase from primary mouse hepatocytes treated with acetaminophen, amiodarone or cyclosporin $A$.

\section{DISCUSSION}

In this study the proteome of primary mouse hepatocytes was assessed for its ability to discriminate between different phenotypes of drug-induced hepatotoxicity, and the level of conservation in response when compared to a human in vitro model was determined.

Three well characterized hepatotoxicants: acetaminophen, amiodarone and cyclosporin A were investigated. Each compound induces a different hepatotoxic phenotype, making it possible to analyze the differences in the pathways which are specific for different classes of hepatotoxicants. Acetaminophen is a analgesic which is safe at a therapeutic dose, but causes severe damage to the liver at higher doses and therefore it is applied as a reference compound for necrosis ${ }^{19}$. Cycloporin $A$ is a immunosuppressive drug which has been shown to 
induce cholestasis ${ }^{20}$. The anti-arrhythmic drug, amiodarone has been shown to induce steatosis as side effect ${ }^{21}$. The primary mouse hepatocytes were exposed for 48 hours to the test compounds and afterwards changes in protein expression were studied. Significantly differentially expressed proteins were only found for cyclosporin A treated cells. The differentially expressed spots were used in a hierarchical clustering analysis where cyclosporin A could be distinguished from acetaminophen and amiodarone. This result is in agreement with our previous study where HepG2 cells were exposed to the same compounds. However, primary mouse hepatocytes show a considerably fewer amount of differentially expressed proteins compared with HepG2 cells, namely 53 spots versus 254 spots ${ }^{14}$. This result was unexpected, since primary hepatocytes have a higher metabolic activity and liver specificity than HepG2 cells ${ }^{22}$. One explanation may be the larger biological variation between the primary mouse hepatocytes compared to HepG2 cells leading to the detection of a smaller number of significantly changed spots.

Furthermore, just like HepG2 the proteome of the primary mouse hepatocytes was not able to make an adequate differentiation between amiodarone, acetaminophen, and the control samples. For each in vitro model, the dosages of the compounds were based on the $\mathrm{IC}_{20}$ concentrations determined with a MTT assay. We used individual $\mathrm{IC}_{20}$ concentrations because, each model can differ in sensitivity to xenobiotic compounds due to their differences in metabolic activity. Probably, the $\mathrm{IC}_{20}$ of acetaminophen and amiodarone induced relatively small effects in vitro which were not detectable with the applied DIGE method. Furthermore, both acetaminophen and amiodarone are indirect toxins, which require biotranformation for toxicity. Acetaminophen is metabolized by the CYP450 enzymes 2E1, 1A2, and 3A4 to its reactive intermediate $\mathrm{N}$-acetyl $p$-benzoquinoneimine, (NAPQI) which is the main metabolite responsible for the toxicity of acetaminophen ${ }^{23,24}$. Amiodarone is extensively metabolized in the liver by CYP3A4 to its toxic mono-N-desethyl and di-N-desethylmetabolites ${ }^{25}$. Although primary mouse hepatocytes are a robust model with drug metabolizing capacities, they do show a decline of the CYP1A2, CYP3A4 and CYP2E1 expression with increased cultivation time ${ }^{6}$. Probably, the decreased expression of these drug metabolizing enzymes leads to less toxic metabolites. So only a relatively small effect can be observed.

We observed 12 differentially expressed proteins that overlap between the primary mouse hepatocytes in the present study and the previously analyzed HepG2 cells (Figure 3.6). These proteins are: Eno1, Hspa5, Tf, Alb, Ces1, Pdia3, Pdia4, Pdia6, Actb, Krt10, Krt8, Eif4a1. 


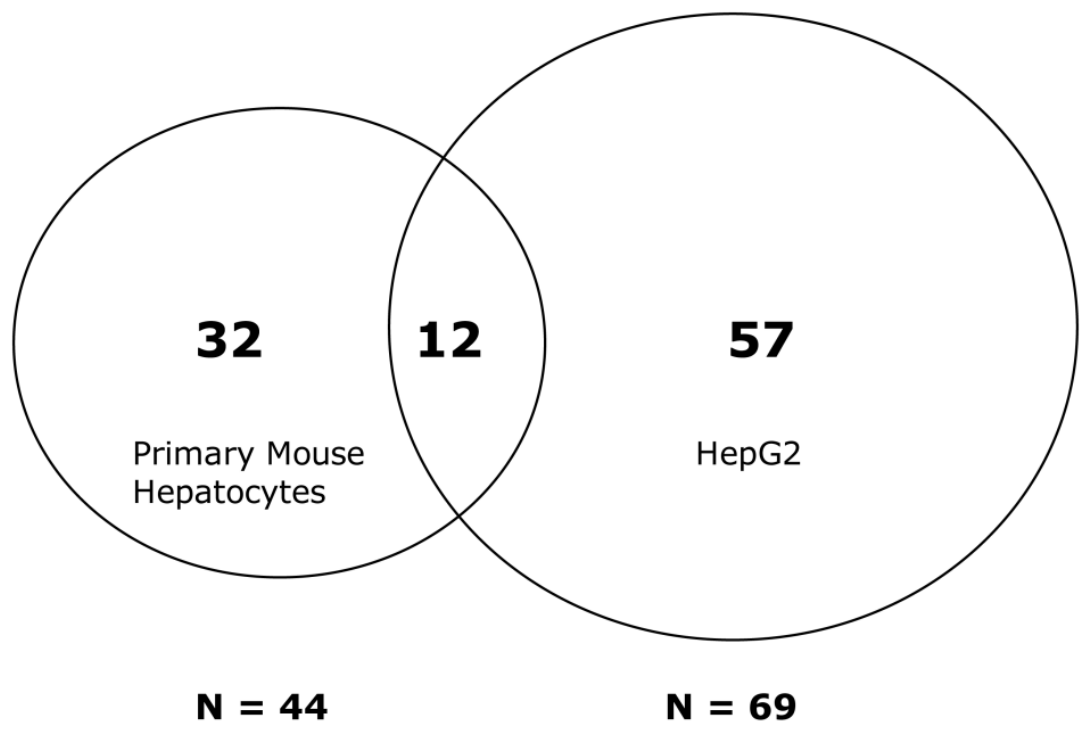

Figure 3.6: Venn diagram of the significant differentially expressed proteins in HepG2 and primary mouse hepatocytes. Overlaps contain the number of significant differentially expressed proteins in both systems induced by the used hepatotoxicants.

Moreover, a classification based on the GO-terms of the differentially expressed proteins with the Panther classification system (http://www.pantherdb.org) revealed a similar outcome (Figure 3.4). Although, different sets of proteins were found differentially expressed, the same pathways in both in vitro systems seem to be affected by cyclosporin A. This strongly indicates that the protein expression induced by the hepatotoxicants, cyclosporin $A$ in particular is conserved in primary mouse hepatocytes when compared to HepG2 cells.

Cyclosporin $A$ is a strong immunosuppressant and induces cholestasis as adverse reaction ${ }^{26}$. It is known that cyclosporin $A$ inhibits the bile salt export pump (ABCB11), multidrug resistance protein 2 ( $A B C C 2)$ and P-glycoprotein (ABCB1) in canalicular membrane vesicles. These ATP Binding Cassette transporters ( $A B C$ transporters) are responsible for the secretion of bile components into the bile canaliculus ${ }^{27}$. Therefore, inhibition of these transport proteins will hamper the bile secretion, which results in cholestasis ${ }^{28}$.

Production and secretion of bile acids is the major route for the elimination of excessive cholesterol ${ }^{29}$. Consequently, cyclosporin A not only induces the accumulation of bile acids but it also increases the hepatic pool of free cholesterol. Excess of free cholesterol is esterified with long-chain fatty acids by acyl-CoA cholesterol acyltransferase-2 (ACAT2). These cholesteryl esters either establish as a part of the neutral lipid core of very low-density lipoprotein (VLDL) or are accumulated as cytoplasmic lipid droplets ${ }^{29}$.

Previously, it was shown that cyclosporin A induced an increase of hepatic VLDL triglyceride secretion ${ }^{30}$, and a decrease of high-density lipoprotein (HDL) plasma levels. The secretion of VLDL, containing cholesteryl esters, might be an alternative pathway for the removal of excessive cholesterol. Consistent with an increased triglyceride secretion is our observation that 
Plin 2, a major protein for storage of triglycerides, was found decreased after a cyclosporin A treatment. In adipocytes it was also shown that a decreased expression of Plin 2 was accompanied with an increased secretion of VLDL ${ }^{31}$.

The cholesteryl esters, stored in intracellular lipid droplets, can be hydrolyzed by Ces1. So they become available for bile acid synthesis ${ }^{29}$. Here, cyclosporin $A$ induced a downregulation of Ces1 on the proteome and transcriptome of primary mouse hepatocytes. A decreased expression of Ces1 will lower the amount of free cholesterol and subsequently decrease the amount of bile acids in the hepatotocytes, which can be seen as a protective adaptive response against cyclosporin A-induced cholestasis.

The phase II detoxifying enzymes are responsible for another mechanism to lower the amount of intracellular bile acids. By means of a conjugation reaction (e.g. sulfation, acetylation and glucuronidation) these biotransformation enzymes convert xenobiotics or endogenous products into more easily excretable substances ${ }^{32}$.

Our study shows an increased expression of the Sult2A1 as a response to cyclosporin A treatment. This phase II detoxifying enzyme catalyzes the sulfation of steroids and bile acids in the liver to increase their polarity and to enhance renal and fecal excretion ${ }^{33}$. Consequently, sulfation of bile salts is linked to a possible protective mechanism against monohydroxy bile salts $^{34}$.

Two forms of glutathione-S-transferase (Gsta3 and GSTT1) were found down-regulated at the mRNA level, but up-regulated at the protein level. The difference in protein expression may indicate protein modifications of these enzymes. One could expect an increased expression of these phase II detoxifying enzymes in response to chemical compounds. However also rats treated with cyclosporin A, showed a decreased mRNA expression of hepatic glutathione-Stransferase and two glutathione producing enzymes ( $\gamma$-glutamylcysteine synthetase heavy and light chain) ${ }^{35}$.

The selenium-binding (Selenbp 2) protein, a major target of reactive acetaminophen metabolites, was also differentially expressed. Fountoulakis et al. ${ }^{36}$ reported a decrease of Selenbp 2 after an acetaminophen treatment. In this study we also found an acetaminophen-mediated decreased expression of Selenbp 2 (fold change of -1.22, although not significant in the multiple comparison test). However, Selenbp 2 did not only respond to acetaminophen treatment, the same decrease was also found after cyclosporin A treatment of primary mouse hepatocytes. Previously, Selenbp 2 was found down-regulated in the livers of $\mathrm{CCl} 4$ treated mice, a model for liver fibrosis and in $\left(\mathrm{Abc4}^{-} \mathrm{J}^{-}\right)$mice, a model for sclerosing cholangiocytes ${ }^{37}$. Moreover, a reduced level of selenium in serum and an increased concentration hepatic selenium were earlier already linked to cholestasis ${ }^{38,39}$.

Similar to our previous study ${ }^{14}$ several proteins related to ER stress were identified. For example, the ER stress marker $78 \mathrm{kDa}$ glucose-regulated protein was found up-regulated, after cyclosporin A treatment. In addition, the protein disulfide-isomerases A3, A4 and A6 were differentially expressed. Notably, the changes in these proteins were all accompanied by 
transcriptional up-regulation. Protein disulfide-isomerases are important enzymes for proper protein-folding, for this purpose they work in close collaboration with cyclophilins. Cyclophilins are peptidyl-prolyl-trans isomerases, enzymes that accelerate or slow down steps in the folding of proteins. Moreover they are involved in the protein quality control in the ER of living cells ${ }^{40}$. Cyclosporin A is known to be a specific inhibitor of the cyclophilin family. Previously, cyclosporin A was found to translocate cyclophilin B from the ER and promote its secretion ${ }^{41}$. This leads to a decreased expression of cyclophilin $B$ in the cell and an increased expression of cyclophilin $B$ in the secretome ${ }^{42}$. Here, we observed a decreased expression of cyclophilin B, while its transcription is up-regulated. This was also observed for the other proteins involved in ER stress and protein-folding. A disturbed ER-function may be accompanied by an altered secretion from hepatocytes. Indeed, as in the HepG2 cells, evidence of an altered ER-golgi transport was found in the primary mouse hepatocytes. Since cyclosporin A induced the differential expression of Sec14/2. This protein is known to both regulate lipid metabolism and trans-Golgi pathways ${ }^{43}$. It may regulate cholesterol biosynthesis by increasing the transfer of squalene to a metabolic active pool in the cell ${ }^{44}$. In our previous HepG2 study a remarkable amount of secretory proteins were found up-regulated in the cellular protein fraction. In primary mouse hepatocytes we now also found an increased expression of the serum proteins albumin and serotransferrin. The accumulation of unfolded proteins causing ER stress can cause $\mathrm{Ca}^{2+}$ release. High $\mathrm{Ca}^{2+}$ concentrations mediate the transfer of an apoptosis signal to mitochondria ${ }^{45}$.

Increased $\mathrm{Ca}^{2+}$ concentrations will lead to the opening of the mitochondrial permeability transition pore (MPTP). The MPTP is formed by the interaction of the voltage-dependent anionselective channel protein (VDAC) with cyclophilin D and adenine nucleotide translocase ${ }^{46}$. The opening of the permeability transition pore increases the mitochondrial permeability. This results in an increased osmolar load, mitochondrial swelling and eventually rupture of the outer membrane, initiating apoptosis. Previously, it was shown that the permeability transition pore is inhibited by cyclosporin $A$ due to its interaction with cyclophilin $D$, and thereby protecting the cell from apoptosis ${ }^{46}$. Here VDAC2 was up-regulated after cyclosporin A treatment. Probably this may be an attempt to correct for the inhibition of the MPTP by cyclosporin A.

Mitochondrial dysfunction was also indicated by the differential expression of several enzymes of the urea cycle like: ornithine carbamoyl transferase, arginosuccinate synthetase and carbamoylphosphate synthase. Ornithine carbamoyl transferase is responsible for the synthesis of citrulline out of the substrates ornithine and carbamoylphosphate. In the 2-DE proteome map a significant up-regulation of this protein after cyclosporin A treatment was detected, whereas the same condition resulted in a remarkably down regulated expression of the mRNA corresponding to this protein. An altered urea cycle is often seen at patients with liver disease, leading to a reduced capacity to detoxify ammonia resulting in hyperammonemia ${ }^{47}$. 


\section{CONCLUSION}

In conclusion, using primary mouse hepatocytes, as well as HepG2 we were able to distinguish cyclosporin A from control, as well as acetaminophen and amiodarone-treated samples. However, just like for HepG2, the proteome of primary mouse hepatocytes did not allow us to make an adequate differentiation between acetaminophen, amiodarone and the control. We believe this may be due to the decreased expression of certain biotransformation enzymes.

Cyclosporin A induced a different sets of deregulated proteins in the two models, however these belong to similar pathways. Again, the differential expression of proteins related to cyclosporin A induced ER stress and the ER-Golgi transport, which may alter vesicle mediated transport and protein secretion. These similar pathways indicate that cyclosporin A induces a response which is conserved in primary mouse hepatocytes when compared to the HepG2 cells.

Several findings in this study suggest that the differential protein expression pattern seen with cyclosporin $A$, is related to protective mechanisms to cholestasis, such as the expression of Ces 1 and phase II detoxifying enzymes. This study does not only show that both models can be used to analyze the cholestatic properties of cyclosporin A, but also indicates the conserved response of primary mouse hepatocytes compared to HepG2 cells.

\section{ACKNOWLEDGMENTS}

We thank Erik Royackers from the Biomedical Research Institute of Hasselt University for his technical support of the LC MS/MS analysis. We also like to thank Karen Brauers and Charly John from Maastricht University for their technical assistance in the microarray analysis.

\section{REFERENCES}

1 Hewitt, N. J. et al. Primary hepatocytes: current understanding of the regulation of metabolic enzymes and transporter proteins, and pharmaceutical practice for the use of hepatocytes in metabolism, enzyme induction, transporter, clearance, and hepatotoxicity studies. Drug Metab Rev 39, 159-234 (2007).

2 Seglen, P. O. Preparation of isolated rat liver cells. Methods Cell Biol 13, 29-83 (1976).

3 Jennen, D. G. et al. Biotransformation pathway maps in WikiPathways enable direct visualization of drug metabolism related expression changes. Drug Discov Today 15, 851-858 (2010).

4 Donato, M. T., Lahoz, A., Castell, J. V. \& Gomez-Lechon, M. J. Cell lines: a tool for in vitro drug metabolism studies. Curr Drug Metab 9, 1-11 (2008).

5 Boess, F. et al. Gene expression in two hepatic cell lines, cultured primary hepatocytes, and liver slices compared to the in vivo liver gene expression in rats: possible implications for toxicogenomics use of in vitro systems. Toxicol Sci 73, 386-402 (2003).

6 Mathijs, K. et al. Assessing the metabolic competence of sandwich-cultured mouse primary hepatocytes. Drug Metab Dispos 37, 1305-1311 (2009).

7 van Kesteren, P. C. et al. Deregulation of cancer-related pathways in primary hepatocytes derived from DNA repair-deficient Xpa-/-p53+/- mice upon exposure to benzo[a]pyrene. Toxicol Sci 123, 123-132 (2011). 
8 Schoonen, W. G., de Roos, J. A., Westerink, W. M. \& Debiton, E. Cytotoxic effects of 110 reference compounds on HepG2 cells and for 60 compounds on HeLa, ECC-1 and CHO cells. II mechanistic assays on NAD(P)H, ATP and DNA contents. Toxicol In vitro 19, 491-503 (2005).

9 O'Brien, P. J. et al. High concordance of drug-induced human hepatotoxicity with in vitro cytotoxicity measured in a novel cell-based model using high content screening. Arch Toxicol 80, 580-604 (2006).

10 Knasmuller, S. et al. Use of human-derived liver cell lines for the detection of environmental and dietary genotoxicants; current state of knowledge. Toxicology 198, 315-328 (2004).

11 Wilkening, S. \& Bader, A. Influence of culture time on the expression of drug-metabolizing enzymes in primary human hepatocytes and hepatoma cell line HepG2. J Biochem Mol Toxicol 17, 207-213 (2003).

12 Aardema, M. J. \& MacGregor, J. T. Toxicology and genetic toxicology in the new era of "toxicogenomics": impact of "-omics" technologies. Mutat Res 499, 13-25 (2002).

13 Greenbaum, D., Colangelo, C., Williams, K. \& Gerstein, M. Comparing protein abundance and mRNA expression levels on a genomic scale. Genome Biol 4, 117 (2003).

14 Van Summeren, A. et al. Proteomics Investigations of Drug-Induced Hepatotoxicity in HepG2 Cells. Toxicol Sci 120, 109-122 (2011).

15 Mosmann, T. Rapid colorimetric assay for cellular growth and survival: application to proliferation and cytotoxicity assays. J Immunol Methods 65, 55-63 (1983).

16 Bouwman, F. G. et al. The Physiologic Effects of Caloric Restriction Are Reflected in the in vivo AdipocyteEnriched Proteome of Overweight/Obese Subjects. J Proteome Res 8, 5532-5540 (2009).

17 Dumont, D., Noben, J. P., Raus, J., Stinissen, P. \& Robben, J. Proteomic analysis of cerebrospinal fluid from multiple sclerosis patients. Proteomics 4, 2117-2124 (2004).

18 Mathijs, K. et al. Discrimination for genotoxic and nongenotoxic carcinogens by gene expression profiling in primary mouse hepatocytes improves with exposure time. Toxicol Sci 112, 374-384 (2009).

19 Murray, K. F., Hadzic, N., Wirth, S., Bassett, M. \& Kelly, D. Drug-related hepatotoxicity and acute liver failure. J Pediatr Gastroenterol Nutr 47, 395-405 (2008).

20 Rotolo, F. S., Branum, G. D., Bowers, B. A. \& Meyers, W. C. Effect of cyclosporine on bile secretion in rats. Am J Surg 151, 35-40 (1986).

21 Fromenty, B. et al. Amiodarone inhibits the mitochondrial beta-oxidation of fatty acids and produces microvesicular steatosis of the liver in mice. J Pharmacol Exp Ther 255, 1371-1376 (1990).

22 Gerets, H. H. et al. Characterization of primary human hepatocytes, HepG2 cells, and HepaRG cells at the mRNA level and CYP activity in response to inducers and their predictivity for the detection of human hepatotoxins. Cell Biol Toxicol (2012).

23 Patten, C. J. et al. Cytochrome P450 enzymes involved in acetaminophen activation by rat and human liver microsomes and their kinetics. Chem Res Toxicol 6, 511-518 (1993).

24 Bessems, J. G. \& Vermeulen, N. P. Paracetamol (acetaminophen)-induced toxicity: molecular and biochemical mechanisms, analogues and protective approaches. Crit Rev Toxicol 31, 55-138 (2001).

25 Zahno, A. et al. The role of CYP3A4 in amiodarone-associated toxicity on HepG2 cells. Biochem Pharmacol 81 432-441 (2010).

26 Belin, M. W., Bouchard, C. S. \& Phillips, T. M. Update on topical cyclosporin A. Background, immunology, and pharmacology. Cornea 9, 184-195 (1990).

27 Trauner, M. \& Boyer, J. L. Bile salt transporters: molecular characterization, function, and regulation. Physiol Rev 83, 633-671 (2003).

28 Alrefai, W. A. \& Gill, R. K. Bile acid transporters: structure, function, regulation and pathophysiological implications. Pharm Res 24, 1803-1823 (2007).

29 Zhao, B., Natarajan, R. \& Ghosh, S. Human liver cholesteryl ester hydrolase: cloning, molecular characterization, and role in cellular cholesterol homeostasis. Physiol Genomics 23, 304-310 (2005).

$30 \mathrm{Wu}$, J., Zhu, Y. H. \& Patel, S. B. Cyclosporin-induced dyslipoproteinemia is associated with selective activation of SREBP-2. Am J Physiol 277, E1087-1094 (1999).

31 Magnusson, B. et al. Adipocyte differentiation-related protein promotes fatty acid storage in cytosolic triglycerides and inhibits secretion of very low-density lipoproteins. Arterioscler Thromb Vasc Biol 26, 1566-1571 (2006). 
32 Jancova, P., Anzenbacher, P. \& Anzenbacherova, E. Phase II drug metabolizing enzymes. Biomed Pap Med Fac Univ Palacky Olomouc Czech Repub 154, 103-116 (2010).

33 Chen, L. J. \& Segel, I. H. Purification and characterization of bile salt sulfotransferase from human liver. Arch Biochem Biophys 241, 371-379 (1985).

34 Cowen, A. E., Korman, M. G., Hofmann, A. F. \& Cass, O. W. Metabolism of lethocholate in healthy man. I. Biotransformation and biliary excretion of intravenously administered lithocholate, lithocholylglycine, and their sulfates. Gastroenterology 69, 59-66 (1975).

35 Bramow, S. et al. Cholestasis and regulation of genes related to drug metabolism and biliary transport in rat liver following treatment with cyclosporine A and sirolimus (Rapamycin). Pharmacol Toxicol 89, 133-139 (2001).

36 Fountoulakis, M. et al. Two-dimensional database of mouse liver proteins: changes in hepatic protein levels following treatment with acetaminophen or its nontoxic regioisomer 3-acetamidophenol. Electrophoresis $\mathbf{2 1}$, 2148-2161 (2000).

37 Henkel, C. et al. Changes of the hepatic proteome in murine models for toxically induced fibrogenesis and sclerosing cholangitis. Proteomics 6, 6538-6548 (2006).

38 Singh, S., Shackleton, G., Ah-Sing, E., Chakraborty, J. \& Bailey, M. E. Antioxidant defenses in the bile duct-ligated rat. Gastroenterology 103, 1625-1629 (1992).

39 Aaseth, J., Thomassen, Y., Aadland, E., Fausa, O. \& Schrumpf, E. Hepatic retention of copper and selenium in primary sclerosing cholangitis. Scand J Gastroenterol 30, 1200-1203 (1995).

40 Bernasconi, R. et al. Cyclosporine a-sensitive, cyclophilin B-dependent endoplasmic reticulum-associated degradation. PLoS One 5 (2010).

41 Price, E. R. et al. Cyclophilin B trafficking through the secretory pathway is altered by binding of cyclosporin A. Proc Natl Acad Sci U S A 91, 3931-3935 (1994).

42 Lamoureux, F. et al. Quantitative proteomic analysis of cyclosporine-induced toxicity in a human kidney cell line and comparison with tacrolimus. J Proteomics (2011).

43 Curwin, A. J., Fairn, G. D. \& McMaster, C. R. Phospholipid transfer protein Sec14 is required for trafficking from endosomes and regulates distinct trans-Golgi export pathways. J Biol Chem 284, 7364-7375 (2009).

44 Shibata, N. et al. Supernatant protein factor, which stimulates the conversion of squalene to lanosterol, is a cytosolic squalene transfer protein and enhances cholesterol biosynthesis. Proc Natl Acad Sci U S A 98, 22442249 (2001).

45 Grimm, S. The ER-mitochondria interface: The social network of cell death. Biochim Biophys Acta 1823, 327-334 (2011).

46 Crompton, M., Virji, S. \& Ward, J. M. Cyclophilin-D binds strongly to complexes of the voltage-dependent anion channel and the adenine nucleotide translocase to form the permeability transition pore. Eur J Biochem 258, 729-735 (1998).

47 Olde Damink, S. W., Jalan, R. \& Dejong, C. H. Interorgan ammonia trafficking in liver disease. Metab Brain Dis 24 169-181 (2009). 


\section{CHAPTER 4}

\section{The secretome of primary mouse hepatocytes in drug-induced hepatotoxicity screening}

Anke Van Summeren, Johan Renes, Freek G. Bouwman, Jean-Paul Noben, Joost H. M. van Delft, Jos C. S. Kleinjans and Edwin C. M. Mariman 


\section{ABSTRACT}

The screening of candidate pharmaceuticals for human safety asks for new in vitro models which detect drug-induced hepatotoxicity more accurate and earlier in the development. Preferably, these models are able to distinguish the different phenotypes of hepatotoxicity including necrosis, cholestasis and steatosis.

Besides the detoxification and elimination of toxic compounds, the liver is responsible for the production and secretion of serum proteins. In this respect, hepatotoxicity can be reflected in the plasma by changes in the levels of secreted liver proteins or by proteins leaking from affected hepatocytes. Therefore, the proteins secreted by hepatocytes are conceivably a rich source of candidate biomarkers for drug-induced hepatotoxicity, both in vitro as well as in vivo.

Here, we analyzed the secretome of primary mouse hepatocytes exposed to three different hepatotoxicants (acetaminophen, amiodarone and cyclosporin A), each reflecting a different hepatotoxic phenotype. Furthermore, we used a secretion blocking strategy by means of a reduction of the incubation temperature to $20^{\circ} \mathrm{C}$, to verify the active secretion of the proteins. The secretome map from non-challenged primary mouse hepatocytes shows a considerable amount of plasma proteins such as albumin, ceruloplasmin and antithrombin-III, indicating that primary mouse hepatocytes preserve well their liver-specific characteristics regarding protein secretion. Cyclosporin A and acetaminophen induced differential secretion of several proteins in the medium which can be related to toxicological mechanisms including an altered cholesterol and lipid metabolism and stress responses. As such, primary mouse hepatocytes can serve as a model to test drug-induced effects on the secretome. 


\section{INTRODUCTION}

The liver is one of the primary targets of drug-induced toxicity. This is because it is responsible for the detoxification and elimination of toxic compounds. Moreover, the liver produces serum proteins such as albumin, serotransferrin, fibrinogen and apolipoproteins which are actively secreted by the hepatocytes ${ }^{1}$. For that reason, aberrantly secreted proteins or proteins leaked from the cells induced by hepatotoxic compounds can serve as potential biomarkers for hepatotoxicity ${ }^{2}$. Secreted proteins are readily accessible through blood and plasma and therefore easy to detect in clinical studies. Examples are current liver function tests, where the activity of several liver enzymes e.g. alanine aminotransferase, aspartate aminotransferase, gamma glutamic transpeptidase, lactic dehydrogenase and alkaline phosphatase is measured in the blood.

Earlier, the cellular and secreted proteome of primary human hepatocytes and of the hepatoma cells HepG2 and Hep3B were compared ${ }^{1}$. As expected, the secretome of the primary human hepatocytes contained the most liver specific proteins, in comparison with the hepatoma cells. It was shown that primary human hepatocytes secrete a considerable amount of plasma proteins including albumin, serotransferrin, apolipoproteins and fibrinogen, which is a characteristic function of hepatocytes ${ }^{1}$.

In a different study, secretome analysis of primary rat hepatocytes revealed that they secrete several plasma proteins and as such indicate their potential for toxicity analysis ${ }^{3}$. These primary rat hepatocytes are often used as an alternative for the primary human hepatocytes since the latter are hindered by scarcity and genetic variation. However, both human and rat primary hepatocytes show a relatively rapid decline of their cytochrome P450 enzyme activity 4. Instead, the gene expression profile and cytochrome P450 enzyme activity of primary mouse hepatocytes showed that they preserve their metabolic competence better than primary rat hepatocytes ${ }^{5}$.

Earlier, we analyzed the cellular proteome of primary mouse hepatocytes after treatment with well-defined hepatotoxicants (acetaminophen, amiodarone and cyclosporin A) ${ }^{6}$. Each compound is reflective for another phenotype of hepatotoxicity; acetaminophen induces necrosis, amiodarone steatosis and cyclosporin A cholestasis. In the cellular proteome of primary mouse hepatocytes, cyclosporin $A$ was found to induce endoplasmic reticulum (ER) stress and an altered ER-Golgi transport which may result in an inhibited protein secretion. This prompted us to analyze the secretome of these primary mouse hepatocytes after administration of the same hepatotoxic compounds. Aiming to detect mechanism-specific candidate biomarkers for drug-induced hepatotoxicity, with respect to the different phenotypes of hepatotoxicity, the secretome was analyzed by difference gel electrophoresis (DIGE). Furthermore, we used a secretion blocking strategy by means of a reduction of the incubation temperature to $20^{\circ} \mathrm{C}$, to verify the active secretion of the proteins ${ }^{7,8}$. In this way genuinely secreted proteins were distinguished from cell-leakage proteins derived from cellular injury. 


\section{MATERIALS AND METHODS}

\section{Chemicals}

Dulbecco's modified Eagle's medium, fetal calf serum, penicillin/streptomycin, Hanks' calcium- and magnesium-free buffer and insulin were obtained from Invitrogen (Breda, The Netherlands). Glucagon, hydrocortisone (water soluble), collagenase type IV, dimethylsulfoxide (DMSO), trypan blue, 3-(4,5-dimethylthiazol-2-yl)-2,5-diphenyltetrazolium bromide, acetaminophen, amiodarone, cyclosporin A (BioChemika) and $\mathrm{N}, \mathrm{N}$-dimethylformamide (anhydrous, $99.8 \%$ ) were purchased from Sigma-Aldrich (Zwijndrecht, The Netherlands). Collagen Type I Rat Tail was obtained from BD BioSciences (Bedford, MA, USA), The Protein Assay Kit was from BioRad (Hercules, CA, USA). All DIGE chemicals were purchased from GE Healthcare (Diegem, Belgium).

\section{Animals}

Permission for animal studies was obtained from the Animal Ethical Committee of Maastricht University, The Netherlands (approval no. 2008-075). Adult male C57/B6 mice (Charles River), weighing 20-25 g, were obtained from Charles River GmbH (Sulzfeld, Germany). This mouse strain was chosen because it is frequently used in toxicological and pharmacological investigations. The animals were housed in macrolon cages with sawdust bedding at $22^{\circ} \mathrm{C}$ and $50-60 \%$ humidity. The light cycle was $12 \mathrm{~h}$ light/12 h dark. Food and tap water were available ad libitum.

\section{Isolation and culturing of primary mouse hepatocytes}

Hepatocytes were isolated by a two-step collagenase perfusion method according to Seglen ${ }^{9}$, with modifications as described before ${ }^{5}$. Cell suspensions with cell viability $\geq 80 \%$, determined by trypan blue exclusion, were brought into culture in a collagen-collagen sandwich formation as described ${ }^{5}$. Prior to treatment, the primary mouse hepatocytes were allowed to recover for $40-42 \mathrm{~h}$ at $37^{\circ} \mathrm{C}$ in a humidified chamber with $95 \%$ air / $5 \% \mathrm{CO} 2$ in serum-free culture medium supplemented with insulin $(0.5 \mathrm{U} / \mathrm{ml})$, glucagon $(7 \mathrm{ng} / \mathrm{ml})$, hydrocortisone $(7.5 \mu \mathrm{g} / \mathrm{ml})$, and $2 \%$ penicillin/streptomycin $(5000 \mathrm{U} / \mathrm{ml}$ penicillin and $5000 \mathrm{U} / \mathrm{ml}$ streptomycin). Culture medium was refreshed every $24 \mathrm{~h}$.

\section{Cytotoxicity Analysis}

To determine the $\mathrm{IC}_{20}$ concentration of the test compounds (acetaminophen, amiodarone or cyclosporin A) an MTT reduction method ${ }^{10}$ was performed. For this, the cells were cultured on collagen pre-coated 6 -well plates at a density of $1.3 \times 10^{6}$ cells/well and exposed for 48 hours to a concentration range of the test compounds (Figure 4.1). 

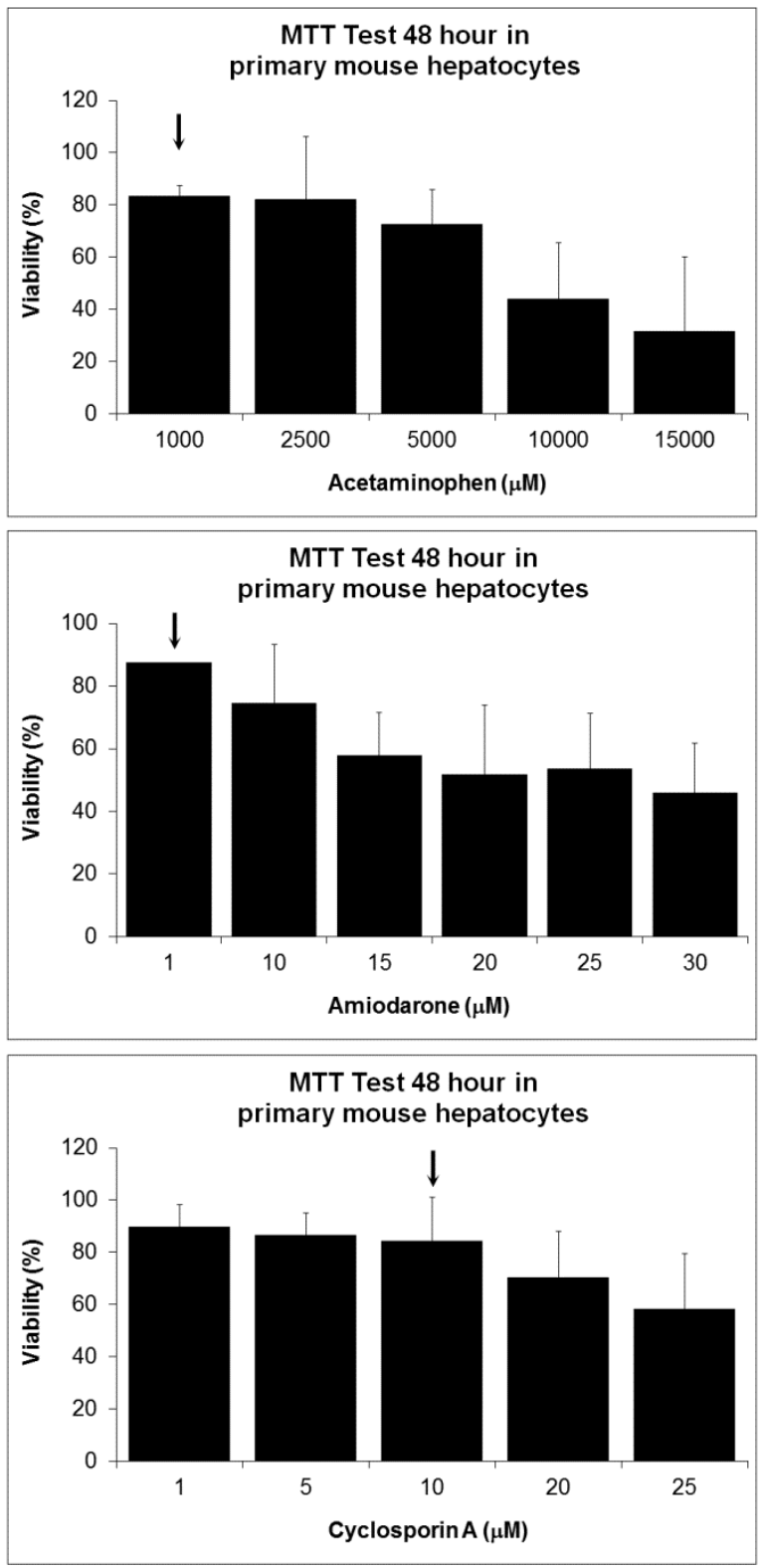

Figure 4.1: MTT test in primary mouse hepatocytes after 48 hours exposure to (A) acetaminophen, (B) amiodarone or $(\mathrm{C})$ cyclosporin $\mathrm{A}$. The arrows indicate the $\mathrm{IC}_{20}$ concentrations of each compound.

\section{Cell treatment}

Cells were cultured on collagen pre-coated petri dishes ( $6 \mathrm{~mm}$ inner diameter) at a density of $2.6 \times 10^{6}$ cells/dish. The hepatocytes were exposed to the $I_{20}$ of the test compounds: 
$1 \mathrm{mM}$ acetaminophen, $1 \mu \mathrm{M}$ amiodarone and $10 \mu \mathrm{M}$ cyclosporin A or $0.5 \%$ DMSO, as a vehicle control. The medium was refreshed and collected after $24 \mathrm{~h}$ and $48 \mathrm{~h}$ of exposure. The experiment contains five independent biological replicas (each from a different animal). Media containing the secreted proteins from each condition were collected from 4 petri dishes with $2.6 \times 10^{6}$ cells.

In addition, to discriminate secreted proteins from cell-leakage proteins a secretion blocking experiment was performed as described ${ }^{8}$. The cells were cultured 16 hours at 20 o $C$ to block protein secretion. The secretome from this condition was compared with the secreted proteins from cells incubated 16 hours at the standard temperature of $37^{\circ} \mathrm{C}$. Here, the cells were obtained from three independent biological experiments; the media from each condition came from 4 petri dishes with $2.6 \times 10^{6}$ cells.

\section{Protein extraction and DIGE}

The media from the cells were centrifuged at $4^{\circ} \mathrm{C}, 230 \mathrm{~g}$ for $10 \mathrm{~min}$, the supernatants were transferred to dialysis tubes (2 kDa molecular weight cut-off, Carl Roth GmbH, Karlsruhne, Germany) and dialyzed against $20 \mathrm{mM}$ ammonium bicarbonate for $48 \mathrm{~h}$ at $4^{\circ} \mathrm{C}$, with at least 8 buffer changes. The dialyzed samples were freeze-dried and dissolved in a DIGE labeling buffer containing $7 \mathrm{M}$ urea, $2 \mathrm{M}$ thiourea, $4 \%(\mathrm{w} / \mathrm{v}) \mathrm{CHAPS}$ and $30 \mathrm{mM}$ Tris- $\mathrm{HCl}$. The protein concentrations were determined with a protein assay kit (Bio-Rad). Aliquots were stored at $-80^{\circ} \mathrm{C}$.

The protein labeling and the DIGE were performed as described before ${ }^{11}$ with the exception that in this study $\mathrm{pH}$ 4-7 IPG strips/buffer were used. For the toxin experiment the gels were analyzed with the DeCyder ${ }^{\mathrm{TM}} 7.0$ software (GE Healthcare) and a two-way ANOVA test (Pvalue $\leq 0.05$ ) was used to select the significantly differential spots between the four treated groups (control, acetaminophen, amiodarone, cyclosporin A), and between the time points. With the DeCyder ${ }^{\mathrm{TM}}$ Extended Data Analysis module, a principle component analysis (PCA) was performed on the differential spots to analyze the correlations between the experimental groups. For the blocking experiment, the gels were analyzed with the DeCyder ${ }^{\mathrm{TM}} 7.0$ software (GE Healthcare). A student T-test ( $P$-value $\leq 0.05)$ was used to select the protein spots which were differentially expressed at $20^{\circ} \mathrm{C}$.

Furthermore, the differentially expressed protein spots from the toxin treatment were verified for protein secretion by comparing them with the protein spots from the secretion blocking experiment.

\section{In gel digestion and protein identification}

The differentially expressed proteins were picked from two preparative gels loaded with $150 \mu \mathrm{g}$ protein mixture from all samples post-stained with Sypro Ruby. The protein spots were identified by using matrix assisted laser desorption ionization time-of-flight tandem mass spectrometry (MALDI-TOF/TOF MS) as described ${ }^{12}$. Protein spots that could not be 
identified via MALDI-TOF/TOF MS were further analyzed by nano liquid chromatography tandem mass spectrometry (nLC-MSMS) on a LCQ Classic (ThermoFinnigan) as described ${ }^{13}$.

\section{Analysis of Secreted Protein Candidates}

For verification of secreted protein candidates the amino acid sequences were analyzed by means of SignalP 4.1 (http://www.cbs.dtu.dk/services/SignalP/) and SecretomeP 2.0 (http://www.cbs.dtu.dk/services/SecretomeP/). SignalP 4.1 predicts the presence and location of signal peptide cleavage sites in amino acid sequences. SecretomeP 2.0 predicts nonclassical, in particular not signal peptide-triggered protein secretion. In this process the nonclassically secreted proteins should obtain an NN-score above the threshold of 0.5 but not at the same time be predicted to contain a signal peptide. 
Table 4.1: secreted proteins from primary mouse hepatocytes inhibited after cultivation for 16 hours on $20^{\circ} \mathrm{C}$.

$\infty$

\begin{tabular}{|c|c|c|c|c|c|c|c|c|}
\hline No. & $\begin{array}{c}\text { Uniprot } \\
\text { Accession } \\
\text { No. }\end{array}$ & $\begin{array}{l}\text { Gene } \\
\text { name }\end{array}$ & protein description & $\begin{array}{l}\text { P-value } \\
\text { (T-test) }\end{array}$ & $\begin{array}{c}\text { Fold } \\
\text { change }\end{array}$ & $\begin{array}{c}\text { Presence } \\
\text { of signal } \\
\text { peptide }\end{array}$ & $\begin{array}{c}\text { Non-classically } \\
\text { secreted proteins } \\
\text { (NN-score) }\end{array}$ & Secreted \\
\hline 73 & Q00897 & Serpina1d & Alpha-1-antitrypsin 1-4 & 0.043 & -1.48 & Yes & 0.864 & Yes \\
\hline 5 & P29699 & Ahsg & Alpha-2-HS-glycoprotein & 0.01 & -1.71 & Yes & 0.713 & Yes \\
\hline 4 & P29699 & Ahsg & Alpha-2-HS-glycoprotein & 0.011 & -1.66 & Yes & 0.713 & Yes \\
\hline 20 & Q9R182 & Angptl3 & Angiopoietin-related protein 3 & 0.082 & -1.59 & Yes & 0.515 & Yes \\
\hline 77 & P32261 & Serpinc1 & Antithrombin-III & 0.00013 & -2.2 & Yes & 0.557 & Yes \\
\hline 76 & P32261 & Serpinc1 & Antithrombin-III & 0.002 & -1.75 & Yes & 0.557 & Yes \\
\hline 32 & Q8C196 & Cps1 & Carbamoyl-phosphate synthase, mitochondrial & 0.0073 & -2.21 & No & 0.460 & No \\
\hline 37 & P06797 & Ctsl & Cathepsin L1 & 0.009 & -3.22 & Yes & 0.686 & Yes \\
\hline 35 & P06797 & Ctsl & Cathepsin L1 & 0.012 & -3.29 & Yes & 0.686 & Yes \\
\hline 36 & P06797 & Ctsl & Cathepsin L1 & 0.036 & -2.04 & Yes & 0.686 & Yes \\
\hline 31 & Q61147 & $\mathrm{Cp}$ & Ceruloplasmin & 0.021 & -1.64 & Yes & 0.632 & Yes \\
\hline 28 & Q61147 & $\mathrm{Cp}$ & Ceruloplasmin & 0.023 & -1.83 & Yes & 0.632 & Yes \\
\hline 29 & Q61147 & $\mathrm{Cp}$ & Ceruloplasmin & 0.023 & -1.56 & Yes & 0.632 & Yes \\
\hline 30 & Q61147 & $\mathrm{Cp}$ & Ceruloplasmin & 0.039 & -1.55 & Yes & 0.632 & Yes \\
\hline 27 & P01027 & $\mathrm{C} 3$ & Complement C3 precursor & 0.0023 & -1.72 & Yes & 0.534 & Yes \\
\hline 26 & P01027 & $\mathrm{C} 3$ & Complement C3 precursor & 0.013 & -2.67 & Yes & 0.534 & Yes \\
\hline 44 & Q9QXC1 & Fetub & Fetuin-B & 0.0065 & -1.56 & Yes & 0.582 & Yes \\
\hline 62 & P47876 & Igfbp1 & Insulin-like growth factor binding protein 1 & 0.0051 & -3.04 & Yes & 0.693 & Yes \\
\hline 60 & P47876 & Igfbp1 & Insulin-like growth factor binding protein 1 & 0.0081 & -2.73 & Yes & 0.693 & Yes \\
\hline 65 & Q5FW60 & Mup20 & Major urinary protein 20 & 0.0076 & -2.04 & Yes & 0.877 & Yes \\
\hline 66 & Q5FW60 & Mup20 & Major urinary protein 20 & 0.017 & -2.07 & Yes & 0.877 & Yes \\
\hline 19 & Q07456 & AMBP & Protein AMBP & 0.012 & -2.28 & Yes & 0.767 & Yes \\
\hline 16 & P07724 & Alb & Serum albumin & 0.036 & -1.57 & Yes & 0.553 & Yes \\
\hline 10 & P07724 & Alb & Serum albumin & 0.086 & -1.48 & Yes & 0.553 & Yes \\
\hline 52 & P21614 & $\mathrm{Gc}$ & Vitamin D-binding protein & 0.0014 & -2.54 & Yes & 0.595 & Yes \\
\hline 47 & P21614 & $\mathrm{Gc}$ & Vitamin D-binding protein & 0.005 & -2.01 & Yes & 0.595 & Yes \\
\hline 49 & P21614 & $\mathrm{Gc}$ & Vitamin D-binding protein & 0.014 & -2.25 & Yes & 0.595 & Yes \\
\hline 51 & P21614 & $\mathrm{Gc}$ & Vitamin D-binding protein & 0.043 & -1.78 & Yes & 0.595 & Yes \\
\hline 48 & P21614 & $\mathrm{Gc}$ & Vitamin D-binding protein & 0.0034 & -2.33 & Yes & 0.595 & Yes \\
\hline
\end{tabular}




\section{RESULTS}

\section{Characterization of the secretome of primary mouse hepatocytes}

To analyze the secretome of the primary mouse hepatocytes, the cells were cultured for 16 hours at $37^{\circ} \mathrm{C}$ and at $20^{\circ} \mathrm{C}$. At $20^{\circ} \mathrm{C}$ the ER/Golgi as well as the ER/Golgi-independent secretion pathways are blocked ${ }^{7}$. As such secreted proteins were discriminated from cell-leakage proteins. From a total of 2069 spots, 44 proteins were significantly inhibited (P-value $\leq 0.05$ ) by $20^{\circ} \mathrm{C}$ and 9 spots showed a trend $(0.05<\mathrm{P}$-value $\geq 0.1)$ for a blocked protein secretion (Table 4.1). From these spots, 29 were identified, belonging to 13 different proteins.

Figure 4.2 shows the 2-DE map from the secreted proteins, the identified spots indicated with a red number were inhibited on $20^{\circ} \mathrm{C}$ and corresponds to the numbers used in Table 4.1. By using SignalP 4.1 and SecretomeP 2.0 analysis all proteins except carbamoyl-phosphate synthase, were determined as secreted proteins. These secreted proteins included liver specific proteins such as serum albumin, protein AMBP, ceruloplasmin, fetuin-B, antithrombin-III, angiopoietin-related protein 3 . The secretome map was matched with the 2-DE map from the toxicological experiment to verify secreted differentially expressed proteins.

\section{Differential expression of secreted proteins in treated primary mouse hepatocytes}

To analyze hepatotoxic effects of the compounds: amiodarone, cyclosporin A and acetaminophen; the secretome from treated primary mouse hepatocytes was collected after 24 and 48 hours. Afterwards, the differentially expressed proteins between the treated groups and DMSO as a vehicle control, were analyzed with DIGE.

From a total of 2069 spots 130 proteins were significantly (two-way Anova Condition Treatment P-value $\leq 0.05$ ) differentially expressed by the compounds. Fifty one protein spots were differentially expressed according to time as well as treatment ( $P$-value interaction time and treatment $\leq 0.05$ ). From the 134 spots, we identified 60 spots belonging to 38 different proteins (Table 4.2). From these 38 different proteins 26 were determined as classical secreted proteins (e.g. serum albumin, antithrombin-III, fibrinogen-like protein 1), 5 as non-classical secreted proteins (e.g. selenium-binding protein 2 and cytochrome b5) and 7 proteins were derived from cell death or leakage (e.g. 10-formyltetrahydrofolate dehydrogenase and carbamoyl-phosphate synthase). Figure 4.2 shows the 2-DE map with the identified differential spots indicated by a white number, which corresponds to the numbers presented in Table 4.2. 


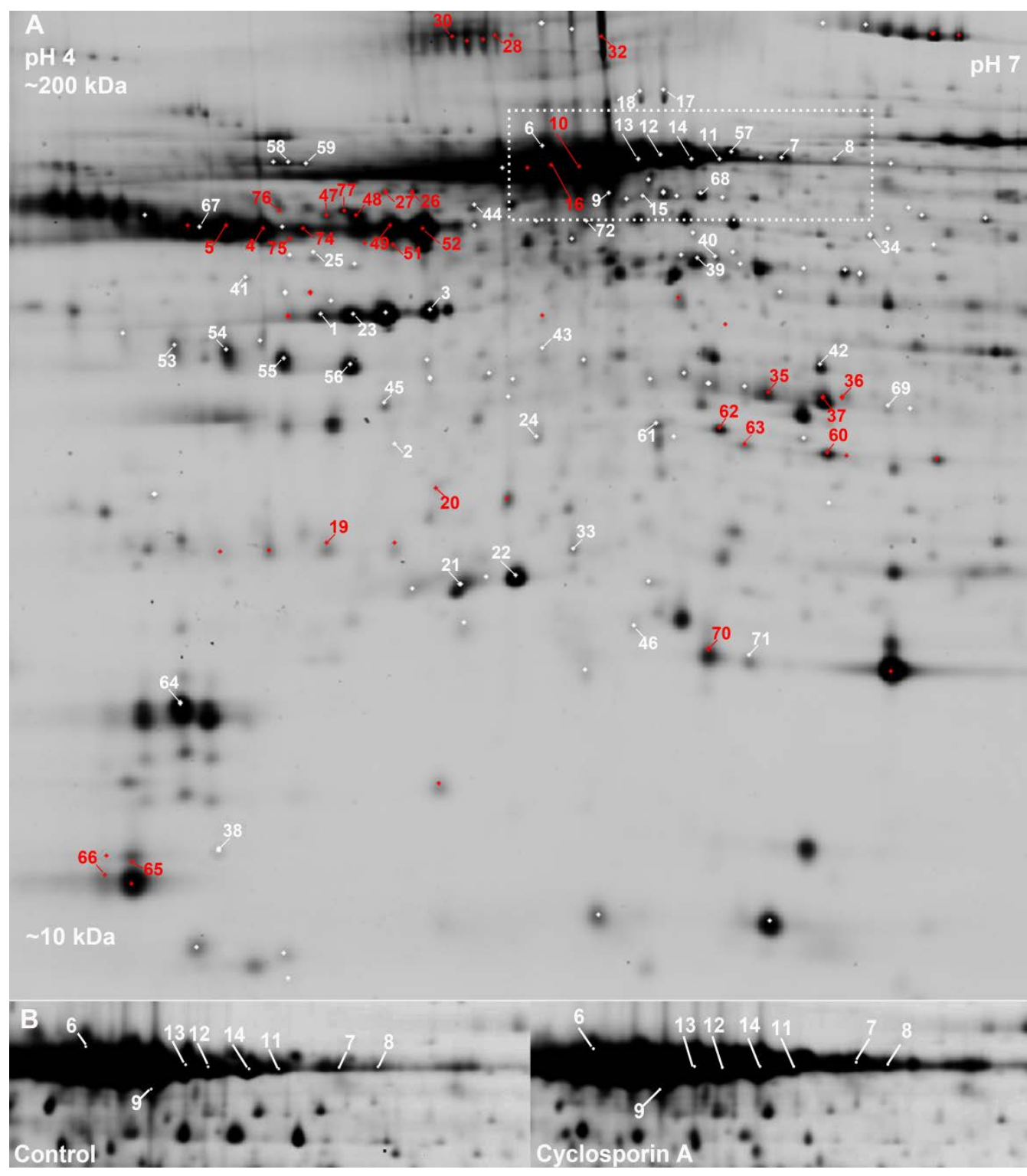

Figure 4.2: (A) Secretome map of the differentially expressed proteins. All differentially expressed spots are shown; the identified spots indicated with a red number are inhibited on $20{ }^{\circ} \mathrm{C}$ and corresponds to the numbers used in Table 4.1. The other spot numbers corresponds with Table 4.2. (B) Detail of the the secretome map with the different isoforms of albumin. 
For all analyzed compounds, 48 hour treatment induced greater differences than 24 hour treatment. A Tukey's multiple comparison test (P-value $\leq 0.05)$ was used to detect significant differences between the control and the different treatment groups. With cyclosporin $A$ treatment, the most differentially expressed proteins were detected. The least differences are discriminated by PC1 with a variance of $17.5 \%$ (Figure 4.3). In PC1, acetaminophen (48 hour) slightly differentiates from the control group (Figure 4.3). On the other hand, cyclosporin A (24 and 48 hours) is clearly distinguished in PC2 (35.6\% of variance), from the control and the other treatments (Figure 4.3).

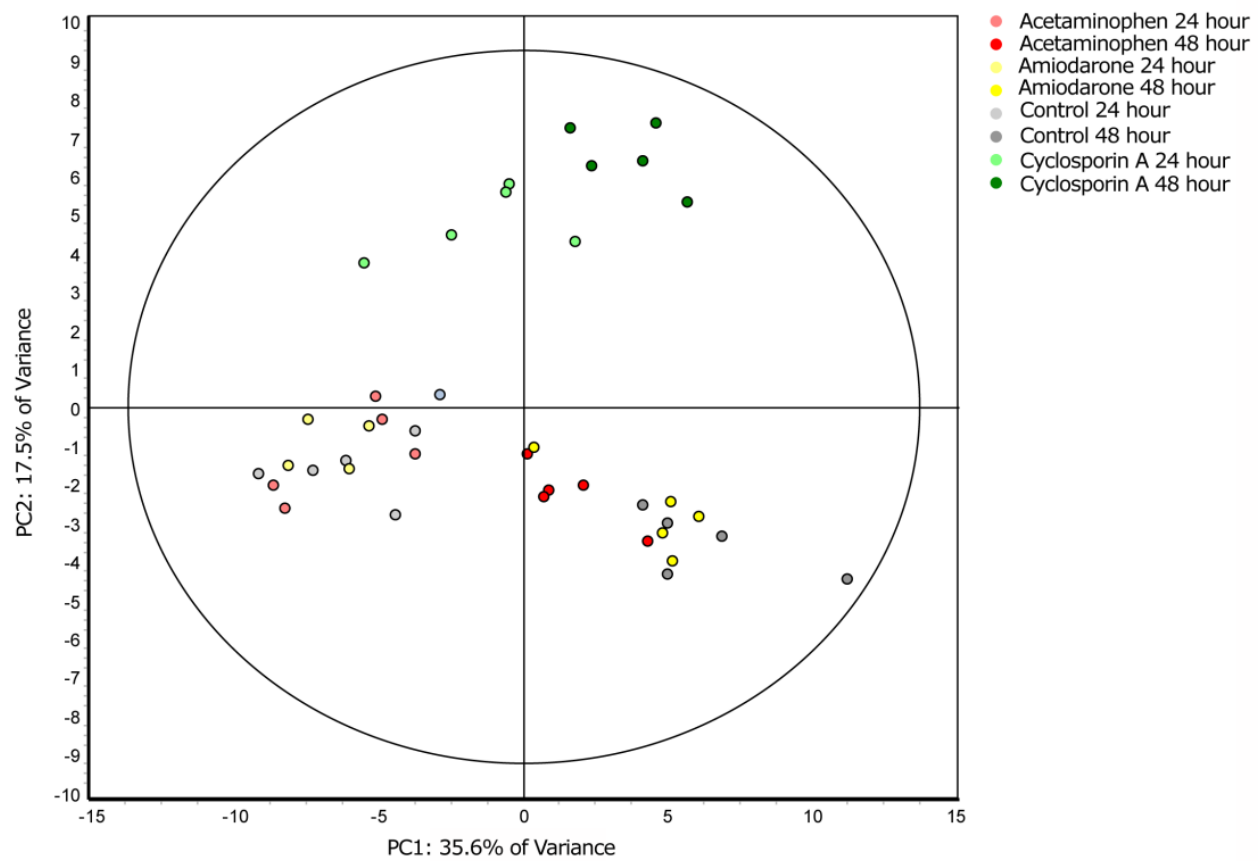

Figure 4.3: PCA analysis of the experimental groups (control, acetaminophen, amiodarone and cyclosporin A), based on the significant differential spots (one-way ANOVA P-value $\leq 0.05$ ), performed with the EDA module of the Decyder software. 
Table 4.2: differentially secreted proteins from primary mouse hepatocytes after exposure to acetaminophen, amiodarone or cyclosporin A.

\begin{tabular}{|c|c|c|c|c|c|c|c|c|c|c|c|c|c|c|}
\hline \multirow{3}{*}{ No. } & \multirow{3}{*}{$\begin{array}{l}\text { Uniprot } \\
\text { Accession } \\
\text { No. }\end{array}$} & \multirow{3}{*}{$\begin{array}{l}\text { Gene } \\
\text { name }\end{array}$} & \multirow{3}{*}{ Protein description } & \multirow{2}{*}{\multicolumn{3}{|c|}{$\begin{array}{c}\text { P-value } \\
\text { Two-way ANOVA }\end{array}$}} & \multicolumn{6}{|c|}{ Fold Change } & \multirow{3}{*}{$\begin{array}{l}\text { Blocked } \\
\text { on } 20^{\circ} \mathrm{C}\end{array}$} & \multirow{3}{*}{$\begin{array}{l}\text { Secre- } \\
\text { ted }\end{array}$} \\
\hline & & & & & & & & 24 hour & & 48 hour & & & & \\
\hline & & & & $\begin{array}{l}\text { Treat- } \\
\text { ment }\end{array}$ & Time & $\begin{array}{l}\text { Interact- } \\
\text { ion }\end{array}$ & Apap/C & Amio/C & $\mathrm{CSA} / \mathrm{C}$ & Apap/C & Amio/C & $\mathrm{CSA} / \mathrm{C}$ & & \\
\hline
\end{tabular}

Acute-phase response

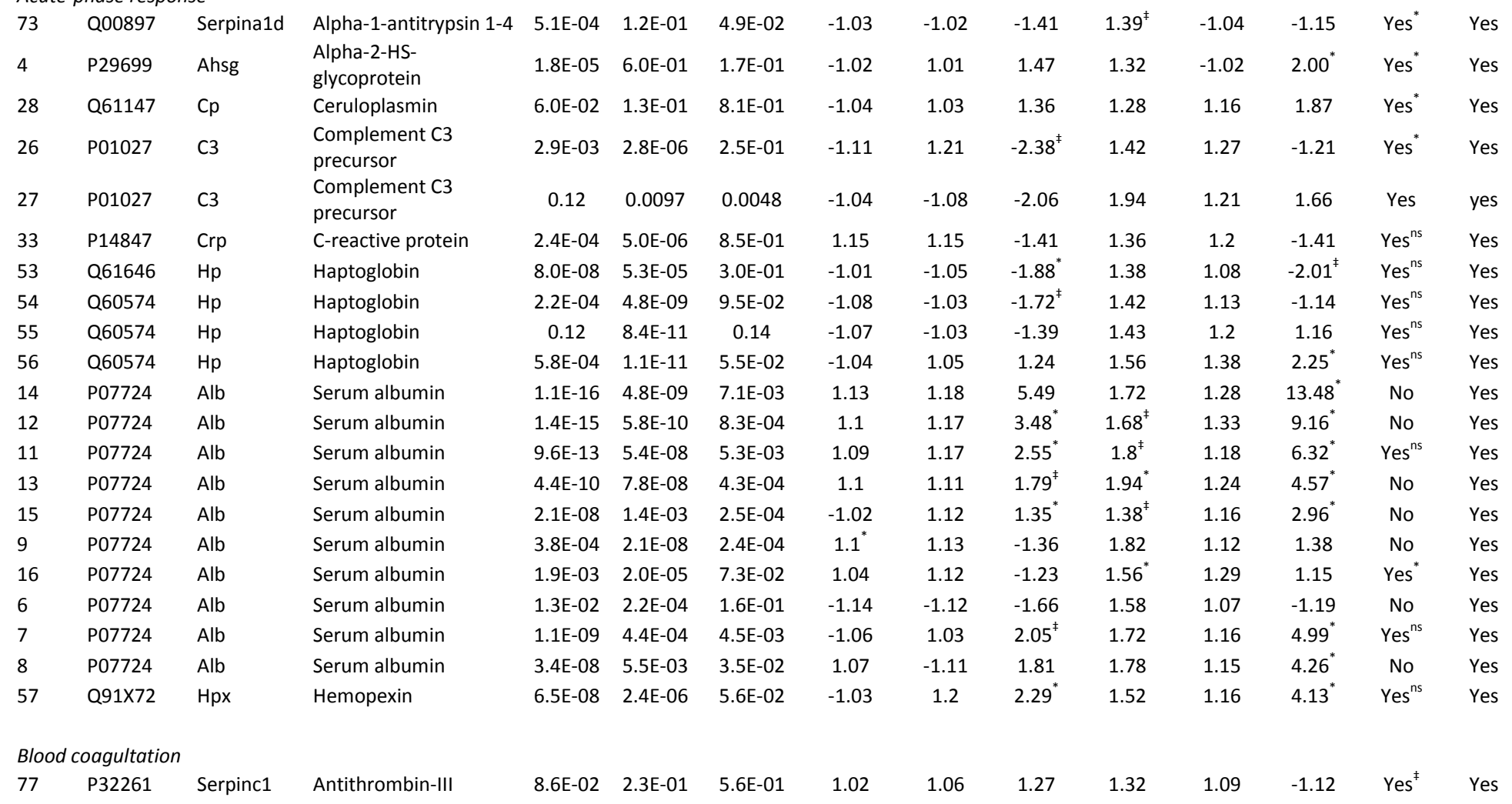




\begin{tabular}{|c|c|c|c|c|c|c|c|c|c|c|c|c|c|c|}
\hline \multirow{3}{*}{ No. } & \multirow{3}{*}{$\begin{array}{l}\text { Uniprot } \\
\text { Accession } \\
\text { No. }\end{array}$} & \multirow{3}{*}{$\begin{array}{l}\text { Gene } \\
\text { name }\end{array}$} & \multirow{3}{*}{ Protein description } & \multirow{2}{*}{\multicolumn{3}{|c|}{$\begin{array}{c}\text { P-value } \\
\text { Two-way ANOVA }\end{array}$}} & \multicolumn{6}{|c|}{ Fold Change } & \multirow{3}{*}{$\begin{array}{l}\text { Blocked } \\
\text { on } 20^{\circ} \mathrm{C}\end{array}$} & \multirow{3}{*}{$\begin{array}{l}\text { Secre- } \\
\text { ted }\end{array}$} \\
\hline & & & & & & & \multicolumn{3}{|c|}{24 hour } & \multicolumn{3}{|l|}{48 hour } & & \\
\hline & & & & $\begin{array}{l}\text { Treat- } \\
\text { ment }\end{array}$ & Time & $\begin{array}{l}\text { Interact- } \\
\text { ion }\end{array}$ & Apap/C & Amio/C & $\mathrm{CSA} / \mathrm{C}$ & Apap/C & Amio/C & $\mathrm{CSA} / \mathrm{C}$ & & \\
\hline 41 & 088947 & F10 & Coagulation factor $\mathrm{X}$ & $3.6 \mathrm{E}-04$ & $1.6 \mathrm{E}-06$ & $9.3 \mathrm{E}-02$ & -1.09 & -1 & $1.57^{\ddagger}$ & -1.3 & 1.09 & 1.43 & $Y_{e s}^{\text {ns }}$ & Yes \\
\hline 45 & Q71KU9 & Fgl1 & $\begin{array}{l}\text { Fibrinogen-like protein } \\
1\end{array}$ & $5.8 \mathrm{E}-03$ & $2.6 \mathrm{E}-08$ & 2.0E-01 & 1.02 & 1.06 & 1.22 & 1 & 1.11 & 1.8 & Yes $^{\text {ns }}$ & Yes \\
\hline \multicolumn{15}{|c|}{ Lipid/cholesterol related processes } \\
\hline 20 & Q9R182 & Angptl3 & $\begin{array}{l}\text { Angiopoietin-related } \\
\text { protein } 3\end{array}$ & $3.8 \mathrm{E}-07$ & 3.7E-06 & $6.0 \mathrm{E}-03$ & 1.16 & 1.21 & $-2.15^{*}$ & $2.22^{*}$ & 1.43 & 1.08 & Yes $^{\ddagger}$ & Yes \\
\hline 21 & Q00623 & ApoA1 & Apolipoprotein A1 & $7.2 \mathrm{E}-06$ & $5.6 \mathrm{E}-07$ & 4.5E-03 & 1.53 & 1.2 & 1.2 & $7.99^{*}$ & 2.19 & $7.26^{*}$ & $Y_{e s}{ }^{n s}$ & Yes \\
\hline 22 & Q00623 & ApoA1 & Apolipoprotein A1 & $1.5 \mathrm{E}-04$ & $5.5 \mathrm{E}-05$ & $5.0 \mathrm{E}-02$ & 1.63 & 1.31 & $1.33^{\ddagger}$ & 5.72 & 2.21 & 6.58 & Yes $^{\text {ns }}$ & Yes \\
\hline 23 & P06728 & ApoA4 & $\begin{array}{l}\text { Apolipoprotein A4 } \\
\text { precursor }\end{array}$ & $1.4 \mathrm{E}-02$ & $5.8 \mathrm{E}-08$ & 4.4E-01 & 1.33 & 1.28 & 1.08 & $2.06^{\ddagger}$ & 1.58 & 1.72 & Yes $^{\text {ns }}$ & Yes \\
\hline 24 & P08226 & ApoE & $\begin{array}{l}\text { Apolipoprotein E } \\
\text { precursor }\end{array}$ & $1.6 \mathrm{E}-02$ & $1.6 \mathrm{E}-05$ & $5.0 \mathrm{E}-01$ & 1.74 & 1.37 & -1.15 & 3.21 & 1.62 & 1.78 & $Y_{e s}{ }^{\text {ns }}$ & Yes \\
\hline 64 & P11589 & Mup2 & $\begin{array}{l}\text { Major urinary protein } \\
2\end{array}$ & $9.8 \mathrm{E}-02$ & 1.7E-12 & $2.9 \mathrm{E}-02$ & 1.04 & -1.07 & -1.12 & 1.65 & 1.35 & 2.02 & $Y_{e s}{ }^{n s}$ & Yes \\
\hline 71 & Q00724 & Rbp4 & $\begin{array}{l}\text { Retinol-binding } \\
\text { protein } 4\end{array}$ & $1.8 \mathrm{E}-02$ & $5.1 \mathrm{E}-02$ & $6.6 \mathrm{E}-01$ & 1.34 & 1.41 & 1.47 & 1.45 & 1.27 & 2.01 & $Y_{e s}{ }^{n s}$ & Yes \\
\hline 50 & P21614 & Gc & $\begin{array}{l}\text { Vitamin D-binding } \\
\text { protein }\end{array}$ & $1.0 \mathrm{E}-05$ & $3.1 \mathrm{E}-03$ & $1.0 \mathrm{E}-02$ & -1.06 & -1.02 & 1.2 & 1.25 & 1.12 & $2.24^{*}$ & $Y_{e s}{ }^{\text {ns }}$ & Yes \\
\hline \multicolumn{15}{|c|}{ Stress } \\
\hline 58 & P20029 & Hspa5 & $\begin{array}{l}78 \mathrm{kDa} \text { glucose- } \\
\text { regulated protein } \\
\text { precursor }\end{array}$ & 4.1E-06 & $6.8 \mathrm{E}-02$ & $1.8 \mathrm{E}-03$ & 1 & -1.21 & 1.2 & -1.17 & 1.03 & $3.73^{*}$ & No & Yes \\
\hline 59 & P20029 & Hspa5 & $\begin{array}{l}78 \mathrm{kDa} \text { glucose- } \\
\text { regulated protein } \\
\text { precursor }\end{array}$ & 1.7E-05 & $5.9 \mathrm{E}-01$ & $2.1 \mathrm{E}-03$ & -1.09 & -1.08 & 1.14 & -1.31 & 1.02 & $3.28^{*}$ & No & Yes \\
\hline 35 & P06797 & Ctsl & Cathepsin L1 & 8.7E-05 & $1.6 \mathrm{E}-01$ & 7.5E-01 & -1.17 & -1.03 & 1.84 & -1.3 & 1.11 & $2.87^{*}$ & Yes $^{*}$ & Yes \\
\hline 37 & P06797 & Ctsl & Cathepsin L1 & $1.5 \mathrm{E}-03$ & $6.0 \mathrm{E}-02$ & 8.5E-01 & -1.27 & -1.14 & 1.63 & -1.56 & -1.09 & 2.35 & Yes $^{\ddagger}$ & Yes \\
\hline 36 & P06797 & Ctsl & Cathepsin L1 & 2.0E-02 & $3.3 \mathrm{E}-03$ & 5.1E-01 & -1.08 & -1.08 & 1.25 & -1.43 & -1.23 & 1.89 & Yes $^{*}$ & Yes \\
\hline 60 & P47876 & Igfbp1 & $\begin{array}{l}\text { Insulin-like growth } \\
\text { factor binding protein } \\
1\end{array}$ & 7.3E-10 & 6.7E-01 & 8.9E-01 & -1.19 & 1.18 & $2.78^{*}$ & -2 & 1.22 & $3.4^{*}$ & Yes $^{*}$ & Yes \\
\hline
\end{tabular}




\begin{tabular}{|c|c|c|c|c|c|c|c|c|c|c|c|c|c|c|}
\hline \multirow{3}{*}{ No. } & \multirow{3}{*}{$\begin{array}{l}\text { Uniprot } \\
\text { Accession } \\
\text { No. }\end{array}$} & \multirow{3}{*}{$\begin{array}{l}\text { Gene } \\
\text { name }\end{array}$} & \multirow{3}{*}{ Protein description } & \multirow{2}{*}{\multicolumn{3}{|c|}{$\begin{array}{c}\text { P-value } \\
\text { Two-way ANOVA }\end{array}$}} & \multicolumn{6}{|c|}{ Fold Change } & \multirow{3}{*}{$\begin{array}{l}\text { Blocked } \\
\text { on } 20^{\circ} \mathrm{C}\end{array}$} & \multirow{3}{*}{$\begin{array}{l}\text { Secre- } \\
\text { ted }\end{array}$} \\
\hline & & & & & & & \multicolumn{3}{|c|}{24 hour } & \multicolumn{3}{|l|}{48 hour } & & \\
\hline & & & & $\begin{array}{l}\text { Treat- } \\
\text { ment }\end{array}$ & Time & $\begin{array}{l}\text { Interact- } \\
\text { ion }\end{array}$ & Apap/C & Amio/C & $\mathrm{CSA} / \mathrm{C}$ & Apap/C & Amio/C & $\mathrm{CSA} / \mathrm{C}$ & & \\
\hline 62 & P47876 & Igfbp1 & $\begin{array}{l}\text { Insulin-like growth } \\
\text { factor binding protein } \\
1\end{array}$ & $2.3 \mathrm{E}-04$ & $6.5 \mathrm{E}-02$ & 4.1E-01 & -1.27 & 1.1 & 1.34 & 1.02 & 1.24 & $2.02^{*}$ & Yes $^{*}$ & Yes \\
\hline 63 & P47876 & Igfbp1 & $\begin{array}{l}\text { Insulin-like growth } \\
\text { factor binding protein } \\
1\end{array}$ & $8.2 \mathrm{E}-13$ & 8.6E-01 & 4.0E-01 & -1.15 & 1.06 & $2.22^{*}$ & 1.03 & 1.28 & $3.34^{*}$ & / & Yes \\
\hline 61 & P47876 & Igfbp1 & $\begin{array}{l}\text { Insulin-like growth } \\
\text { factor binding protein } \\
1\end{array}$ & $1.0 \mathrm{E}-04$ & $2.0 \mathrm{E}-02$ & $8.5 \mathrm{E}-01$ & -1.12 & 1.06 & 1.32 & -1.14 & -1.01 & 1.21 & Yes $^{\text {ns }}$ & Yes \\
\hline 68 & P27773 & Pdia3 & $\begin{array}{l}\text { Protein disulfide- } \\
\text { isomerase A3 } \\
\text { precursor }\end{array}$ & $6.3 \mathrm{E}-09$ & 7.1E-01 & 7.1E-01 & -1.11 & 1.15 & 1.48 & -1.21 & 1.02 & $2.79^{*}$ & No & Yes \\
\hline 67 & P09103 & P4hb & $\begin{array}{l}\text { Protein disulfide- } \\
\text { isomerase precursor }\end{array}$ & $2.1 \mathrm{E}-02$ & $2.6 \mathrm{E}-06$ & $2.5 \mathrm{E}-03$ & 1.01 & 1.02 & -1.05 & $1.34^{\ddagger}$ & 1.03 & $1.46^{*}$ & Yes $^{\text {ns }}$ & Yes \\
\hline 74 & Q03734 & Serpina3m & $\begin{array}{l}\text { Serine protease } \\
\text { inhibitor } \mathrm{A} 3 \mathrm{M}\end{array}$ & $2.4 \mathrm{E}-07$ & $2.0 \mathrm{E}-02$ & $4.2 \mathrm{E}-01$ & -1.07 & -1 & $1.86^{*}$ & 1.15 & -1.08 & $2.38^{*}$ & Yes $^{\text {ns }}$ & Yes \\
\hline 75 & Q91WP6 & Serpina3n & $\begin{array}{l}\text { Serine protease } \\
\text { inhibitor } A 3 N\end{array}$ & $3.5 \mathrm{E}-07$ & $3.1 \mathrm{E}-08$ & $1.2 \mathrm{E}-01$ & 1.04 & 1.19 & 1.81 & 1.31 & -1.1 & $2.96^{*}$ & No & Yes \\
\hline \multicolumn{15}{|c|}{ Non secreted proteins } \\
\hline 17 & Q8R0Y6 & Aldh1l1 & $\begin{array}{l}10- \\
\text { formyltetrahydrofolate } \\
\text { dehydrogenase }\end{array}$ & $6.0 \mathrm{E}-03$ & $1.3 \mathrm{E}-06$ & $6.9 \mathrm{E}-01$ & -1.06 & -1 & 1.79 & -1.29 & -1.1 & 1.25 & No & No \\
\hline 18 & Q8R0Y6 & Aldh1|1 & $\begin{array}{l}10- \\
\text { formyltetrahydrofolate } \\
\text { dehydrogenase }\end{array}$ & 4.3E-02 & $5.9 \mathrm{E}-08$ & $6.5 \mathrm{E}-01$ & -1.07 & 1.05 & 1.56 & -1.17 & -1.03 & 1.12 & No & No \\
\hline 43 & Q920E5 & Fdps & $\begin{array}{l}\text { Farnesyl } \\
\text { pyrophosphate } \\
\text { synthetase }\end{array}$ & $4.6 \mathrm{E}-05$ & 7.6E-06 & $4.1 \mathrm{E}+01$ & 1.02 & 1.16 & $2.09^{*}$ & -1.18 & -1.2 & 1.45 & No & No \\
\hline 2 & P60710 & Actb & Actin, cytoplasmic 1 & $1.3 \mathrm{E}-03$ & $6.9 \mathrm{E}-11$ & 7.4E-01 & 1.07 & 1.03 & 1.29 & 1.03 & 1.06 & $1.53 \ddagger$ & No & No \\
\hline 1 & P60710 & Actb & Actin, cytoplasmic 1 & 4.4E-03 & 2.4E-09 & 1.0E-01 & 1.24 & 1.23 & -1.15 & $2.13^{*}$ & 1.45 & 1.54 & No & No \\
\hline 3 & P60710 & Actb & Actin, cytoplasmic 1 & $4.9 \mathrm{E}-02$ & $9.3 \mathrm{E}-06$ & $9.1 \mathrm{E}-01$ & 1.23 & 1.21 & 1.51 & 1.02 & 1.05 & 1.31 & No & No \\
\hline 32 & Q8C196 & Cps1 & Carbamoyl-phosphate & $1.8 \mathrm{E}-04$ & 2.9E-07 & $5.0 \mathrm{E}-03$ & 1.03 & 1.02 & -1.7 & $2.41^{*}$ & 1.38 & 1.21 & No & No \\
\hline
\end{tabular}




\begin{tabular}{|c|c|c|c|c|c|c|c|c|c|c|c|c|c|c|}
\hline \multirow{3}{*}{ No. } & \multirow{3}{*}{$\begin{array}{l}\text { Uniprot } \\
\text { Accession } \\
\text { No. }\end{array}$} & \multirow{3}{*}{$\begin{array}{l}\text { Gene } \\
\text { name }\end{array}$} & \multirow{3}{*}{ Protein description } & \multirow{2}{*}{\multicolumn{3}{|c|}{$\begin{array}{c}\text { P-value } \\
\text { Two-way ANOVA }\end{array}$}} & \multicolumn{6}{|c|}{ Fold Change } & \multirow{3}{*}{$\begin{array}{l}\text { Blocked } \\
\text { on } 20^{\circ} \mathrm{C}\end{array}$} & \multirow{3}{*}{$\begin{array}{l}\text { Secre- } \\
\text { ted }\end{array}$} \\
\hline & & & & & & & & 24 hour & & 48 hour & & & & \\
\hline & & & & $\begin{array}{l}\text { Treat- } \\
\text { ment }\end{array}$ & Time & $\begin{array}{l}\text { Interact- } \\
\text { ion }\end{array}$ & Apap/C & Amio/C & CSA/C & Apap/C & Amio/C & $\mathrm{CSA} / \mathrm{C}$ & & \\
\hline 34 & Q9DBE0 & Csad & $\begin{array}{l}\text { synthase [ammonia], } \\
\text { mitochondrial } \\
\text { Cysteine sulfinic acid } \\
\text { decarboxylase }\end{array}$ & $9.8 \mathrm{E}-04$ & 3.7E-06 & 4.1E-01 & -1.02 & -1.02 & 1.37 & -1.17 & -1.02 & 1.15 & No & No \\
\hline 39 & P17182 & Eno1 & Alpha-enolase & $2.8 \mathrm{E}-03$ & $6.4 \mathrm{E}-11$ & $3.4 \mathrm{E}-02$ & 1.06 & 1.16 & $2.4^{*}$ & -1.4 & -1.09 & -1.03 & No & No \\
\hline 40 & P17182 & Eno1 & Alpha-enolase & $4.3 \mathrm{E}-02$ & 7.7E-11 & $1.5 \mathrm{E}-01$ & 1.55 & 1.17 & 2.16 & -1.32 & -1.28 & 1.08 & No & No \\
\hline 46 & P29391 & Ftl1 & Ferritin light chain 1 & $5.2 \mathrm{E}-03$ & 2.6E-10 & $5.6 \mathrm{E}-01$ & -1.03 & -1.02 & 1.59 & -1.15 & 1.05 & 1.31 & No & No \\
\hline \multicolumn{15}{|c|}{ Varia } \\
\hline 38 & P56395 & Cyb5 & Cytochrome b5 & 4.0E-03 & $6.0 \mathrm{E}-03$ & $1.4 \mathrm{E}-01$ & -1.07 & -1 & 1.14 & -1.51 & -1.14 & 1.17 & No & NC \\
\hline 72 & Q63836 & Selenbp2 & $\begin{array}{l}\text { Selenium-binding } \\
\text { protein } 2\end{array}$ & $1.1 \mathrm{E}-03$ & 8.0E-08 & 9.7E-01 & -1.03 & 1.02 & 1.59 & -1.03 & $-1.05 \ddagger$ & 1.65 & No & NC \\
\hline 25 & P56480 & Atp5b & $\begin{array}{l}\text { ATP synthase beta } \\
\text { chain, mitochondrial }\end{array}$ & $3.6 \mathrm{E}-03$ & $9.8 \mathrm{E}-01$ & $6.5 \mathrm{E}-01$ & 1.04 & 1.21 & 1.39 & 1.13 & 1.08 & 1.68 & No & $\mathrm{NC}$ \\
\hline 42 & Q9QXD6 & Fbp1 & $\begin{array}{l}\text { Fructose-1,6- } \\
\text { bisphosphatase }\end{array}$ & $2.6 \mathrm{E}-03$ & $3.6 \mathrm{E}-07$ & $3.2 \mathrm{E}-02$ & -1.1 & -1.02 & $2.02^{\ddagger}$ & -1.88 & -1.28 & -1.24 & No & $\mathrm{NC}$ \\
\hline 69 & Q91X91 & Qprt & $\begin{array}{l}\text { Nicotinate-nucleotide } \\
\text { pyrophosphorylase } \\
\text { [carboxylating] }\end{array}$ & $9.6 \mathrm{E}-04$ & $3.8 \mathrm{E}-05$ & $3.8 \mathrm{E}-01$ & 1.02 & -1.06 & $1.88^{\ddagger}$ & -1.58 & -1.25 & 1.38 & No & $\mathrm{NC}$ \\
\hline 19 & Q07456 & AMBP & Protein AMBP & $1.8 \mathrm{E}-05$ & 4.0E-02 & $8.4 \mathrm{E}-01$ & 1.09 & 1.13 & -1.68 & 1.14 & 1.07 & $-1.92^{\ddagger}$ & Yes & Yes \\
\hline
\end{tabular}

${ }^{\ddagger}$ indicates fold changes with a P-value $\leq 0.1$ between the control and the treated group, calculated with a multiple comparison test.

* indicates significant fold changes with a P-value $\leq 0.05$ between the control and the treated group, calculated with a multiple comparison test.

${ }^{\text {ns }}$ Not significant

$\mathrm{NC}=$ Non Classical secreted proteins

/ spot not found 


\section{DISCUSSION}

In the first part of this study the secretome of primary mouse hepatocytes, cultured in a collagen sandwich was characterized by means of a blocking strategy. All identified proteins from the primary mouse hepatocyte secretome map, except carbamoyl-phosphate synthase, are known to be liver secreted proteins. One of the liver responsibilities is the production and secretion of blood proteins including: plasma carriers, apolipoproteins, hemostasis and fibrinolysis factors, and hormones. The identification of known secreted liver proteins demonstrates that primary mouse hepatocytes preserve well their liver-specific characteristics regarding protein secretion. However, it should be noted that carbamoyl-phosphate synthase was found down-regulated at $20^{\circ} \mathrm{C}$, which we cannot explain because carbamoyl-phosphate synthase is known as a mitochondrial protein.

In the second part of the study we analyzed the secretome of primary mouse hepatocytes exposed to three well-characterized hepatotoxic compounds (acetaminophen, amiodarone and cyclosporin A). The secretome of the drug-treated cells revealed several other liver secreted proteins like apolipoproteins A1, A4 and E, c-reactive protein, fibrinogen-like protein 1 and haptoglobulin. Although these proteins were not found significantly down-regulated in the $20^{\circ} \mathrm{C}$ blocking experiment, they could be identified as either classical or non-classical secreted proteins by means of the detection of the signal peptide or NN-score. Hence, the blocking strategy is a good tool to detect secreted proteins; however in primary mouse hepatocytes this strategy is not sufficient for all secreted proteins. Therefore, the detection of a signal peptide or the $\mathrm{NN}$-score seems helpful to distinguish these insufficiently blocked secreted proteins from cytoplasmic proteins.

Previously, the cellular protein expression in HepG2 cells and primary mouse hepatocytes after drug-induced hepatotoxicity was investigated ${ }^{11}$. In HepG2 as well as in the primary mouse hepatocytes most differentially expressed cellular proteins were assigned to cyclosporin A. This was also the case for the secretome of primary mouse hepatocytes. However, unlike the cellular proteome, the secretome of primary mouse hepatocytes was also suitable to detect acetaminophen-induced differentially expressed proteins. In primary rat hepatocytes no toxicity was observed with $1 \mathrm{mM}$ acetaminophen ${ }^{14}$.

According to the cellular proteome, cyclosporin A induced ER stress and altered the ER-Golgi transport. Moreover, proteins that are normally secreted were more abundant in the cellular proteome indicating that they accumulate inside the cell, due to altered secretion pathways ${ }^{11}$. Based on this information, we hypothesized that cyclosporin A may decrease vesicle mediated transport and protein secretion. Indeed, we found decreased secretion of some proteins. However, cyclosporin A also increased the secretion of ApoA1, haptoglobulin, hemopexin and serum albumin which are known as proteins abundantly secreted by the liver. Furthermore, some of these plasma proteins were also up-regulated with acetaminophen or amiodarone. Liver failure is mostly accompanied with a decreased plasma level of serum albumin, while here an increased secretion of serum albumin is observed. The hepatocytes were exposed to 
a relatively low dose of the compounds $\left(\mathrm{IC}_{20}\right)$, so the increased expression of serum albumin might be a low-dose-response leading to increased production and secretion by the cells to scavenge these compounds. Furthermore, differences in the various isoforms of serum albumin are visible, identified as spots $6,7,8,9,11,12,13,14,15$ and 16 (Figure 4.2B). These isoforms appear due to several posttranslational modifications albumin undergoes three major posttranslational modifications, namely: oxidation, glycation and S-nitrosylation ${ }^{15}$. Oxidative modification was detected by increased carbonyl groups and oxidation of cysteine34 in albumin ${ }^{16}$. Previously, progressive oxidative modification of albumin was found with increased of liver failure. Oxidative modifications can be caused by oxidative stress of hepatotoxic compounds like acetaminophen. They can alter the binding capacity of serum albumin transport protein for ions, fatty acids, hormones, bilirubin and drugs. Oxidation of serum albumin of can alter these binding capacities ${ }^{17}$.

Cyclosporin A induced decreased secretion of antithrombin-III, with an increased secretion of fibrinogen and coagulation factor $X$ secretion. Although these differences were not significance in the multiple comparison test, they indicate hyper coagulation. The secretion of coagulation proteins, which is regulated by cellular calcium concentration, indicates cellular damage. To protect the hepatocytes from cellular damage, increased insulin-like growth factor binding protein 1 (Igfbp1) promotes hepatic survival by stimulating liver regeneration ${ }^{18}$. This can explain the increased expression of Igfbp1 induced by acetaminophen and cyclosporin A. It is confirmed by other studies in which igfbp1 was also found up-regulated after acute liver injury ${ }^{19,20}$.

After 48 hours all conditions show, although not always significant, a differential expression of alpha-1-antitrypsin 1-4, alpha-2-HS-glycoprotein, complement C3, c-reactive protein, ceruplasmin, hemopexin, serum albumin and haptoglobulin which are known acute phase response proteins ${ }^{21}$. Acute phase is a clinical state that occurs after many inflammatory stimuli, including xenobiotic stimuli ${ }^{21}$.

The apolipoprotein ApoA1 was up-regulated by both acetaminophen as cyclosporin A. Previously, abnormal plasma lipid and apolipoprotein concentrations were seen in patients with cholestasis but also in non-cholestatic patients with hepatic dysfunction ${ }^{22,23}$. These proteins are important for reverse cholesterol transport, which among others is regulated by regulated by liver $X$ receptors (LXR). LXR are nuclear receptors, which act as cholesterol sensors ${ }^{24}$. In reverse cholesterol transport excess cholesterol from the peripheral tissues is transported back to the liver via high-density lipoproteins (HDL). ApoA1 is the most abundant apolipoprotein in HDL, with its expression being one of the first steps of reverse cholesterol transport.

In the liver, cholesterol is mainly metabolized to bile acids. Consequently, bile acid synthesis is the main route to remove excessive amounts of hepatocellular cholesterol. The metabolism of bile acids is regulated by farnesoid $X$ receptor $(F X R)$, pregnane $X$ receptor $(P X R)$ and vitamin $D$ receptor (VDR). When bile homeostasis is altered, these nuclear receptors initiate an adaptive response to reduce bile acid accumulation and concomitant toxicity ${ }^{25,26}$. Cyclosporin $A$ is known to cause cholestasis through the inhibition of bile salt transport proteins ${ }^{27}$. 
In our study farnesyl pyrophosphate synthetase (Fdps) was up-regulated after 24 hours of cyclosporin A treatment. Fdps is regulated by LXR and catalyzes the formation of farnesyl diphosphate, which is a precursor for several metabolites including sterols, dolichols, carotenoids, and ubiquinones ${ }^{28,29}$. Previously, Fdps together with other enzymes of the mevalonate pathway were also differentially expressed after drug treatment in rats ${ }^{29}$. While bile secretion is inhibited, other sterol efflux pathways are maintained and probably serve as an alternative route to remove excessive cholesterol ${ }^{30}$. Possibly, the production of derivatives from farnesyl diphosphate is in support of these alternative pathways to lower intracellular cholesterol levels.

Besides their role in maintaining cholesterol homeostasis, LXR also regulate fatty acid metabolism ${ }^{24}$. Among others, LXR activate the expression of angiopoietin-like protein $3^{28}$. Angiopoietin-like protein 3 is exclusively expressed and secreted by the liver, and belongs to the family angiopoietin-like proteins. However, its ability to enhance vascular angiogenesis is less than angiopoietin ${ }^{31}$. Previously it was shown that angiopoietin-like protein 3 inactivates lipoprotein lipase and increases plasma triglyceride levels ${ }^{32}$. After 24 hour of treatment with cyclosporin A, secretion of angiopoietein-like protein 3 from primary mouse hepatocytes was significantly down-regulated. In contrast, 48 hour exposure to Acetaminophen, resulted in the up-regulation of angiopoietein-like protein 3.

In our set of secreted proteins, vitamin-D-binding protein was up-regulated in the secretome of the primary mouse hepatocytes after 48 hours exposure of cyclosporin A. This is in line with an in vivo study where rats were treated with hepatotoxic compounds ${ }^{33}$. This in vivo study revealed an increased expression of vitamin-D-binding protein and even suggested it as serum marker for hepatotoxicity ${ }^{33}$. Vitamin $D$ binding protein binds vitamin $D$ and its metabolites ${ }^{34}$. In addition it activates the VDR, which is responsible for the detoxification of secondary bile acids ${ }^{24,35}$. VDR functions as an obligate heterodimer with the retinoic $X$ receptor (RXR) ${ }^{36}$. Both RXR and retinol binding protein 4 participate in the vitamin A and carotenoid metabolism (http://www.wikipathways.org/index.php/Pathway:WP716). Our data-set shows that cyclosporine $A$ increased the secretion of retinol-binding protein 4, although not reaching for significant. Retinol binding protein 4 delivers retinol from the liver stores to the peripheral tissue. Differential expression of retinol-binding protein is associated with liver diseases and drug-induced hepatotoxicity ${ }^{33,37}$. Previously, it was shown that serum retinol-binding protein 4 , positively correlates with the intra hepatic lipid content and insulin resistance ${ }^{38,39}$.

In conclusion, the secretome map of primary mouse hepatocytes at base-line consists of known secreted liver proteins, indicating that primary mouse hepatocytes preserve well their liver-specific characteristics regarding protein secretion.

Treatment with hepatotoxic compounds induced several differentially secreted proteins in the medium which can be related to toxicological mechanisms like an altered cholesterol and lipid metabolism and cellular damage. Fdps, angiopoietin-like protein 3, vitamin-D-binding protein and apolipoprotein A-I all indicate altered cholesterol and lipid metabolism and could be considered as a potential panel of candidate biomarkers for drug-induced hepatotoxicity, 
from which vitamin-D-binding protein and Fdps were specific for cyclosporin A, representing cholestasis. To identify class-specific biomarkers, further research with additional hepatotoxicants is recommended. Afterwards, these candidate biomarkers can be measured in serum of exposed mice with use of quantitative immunoassays.

The present study underscores the value of the secretome analysis from an in vitro model like primary mouse hepatocytes to screen for drug-induced effects.

\section{ACKNOWLEDGMENTS}

We thank Erik Royackers from the Biomedical Research Institute of Hasselt University for his technical support of the nLC-MS/MS analysis. 


\section{REFERENCES}

1 Slany, A. et al. Cell characterization by proteome profiling applied to primary hepatocytes and hepatocyte cell lines Hep-G2 and Hep-3B. J Proteome Res 9, 6-21 (2010).

2 Gray, J. et al. A proteomic strategy to identify novel serum biomarkers for liver cirrhosis and hepatocellular cancer in individuals with fatty liver disease. BMC Cancer 9, 271 (2009).

3 Farkas, D., Bhat, V. B., Mandapati, S., Wishnok, J. S. \& Tannenbaum, S. R. Characterization of the secreted proteome of rat hepatocytes cultured in collagen sandwiches. Chem Res Toxicol 18, 1132-1139 (2005).

4 Boess, F. et al. Gene expression in two hepatic cell lines, cultured primary hepatocytes, and liver slices compared to the in vivo liver gene expression in rats: possible implications for toxicogenomics use of in vitro systems. Toxicol Sci 73, 386-402 (2003).

5 Mathijs, K. et al. Assessing the metabolic competence of sandwich-cultured mouse primary hepatocytes. Drug Metab Dispos 37, 1305-1311 (2009).

6 Van Summeren, A. et al. Screening for drug-induced hepatotoxicity in primary mouse hepatocytes using acetaminophen, amiodarone, and cyclosporin a as model compounds: an omics-guided approach. Omics : a journal of integrative biology 17, 71-83 (2013).

7 Hughes, R. C. Secretion of the galectin family of mammalian carbohydrate-binding proteins. Biochim Biophys Acta 1473, 172-185 (1999).

8 Wang, P. et al. Profiling of the secreted proteins during 3T3-L1 adipocyte differentiation leads to the identification of novel adipokines. Cell Mol Life Sci 61, 2405-2417 (2004).

9 Seglen, P. O. Preparation of isolated rat liver cells. Methods Cell Biol 13, 29-83 (1976).

10 Mosmann, T. Rapid colorimetric assay for cellular growth and survival: application to proliferation and cytotoxicity assays. J Immunol Methods 65, 55-63 (1983).

11 Van Summeren, A. et al. Proteomics Investigations of Drug-Induced Hepatotoxicity in HepG2 Cells. Toxicol Sci 120, 109-122 (2011).

12 Bouwman, F. G. et al. The Physiologic Effects of Caloric Restriction Are Reflected in the in Vivo AdipocyteEnriched Proteome of Overweight/Obese Subjects. J Proteome Res 8, 5532-5540 (2009).

13 Dumont, D., Noben, J. P., Raus, J., Stinissen, P. \& Robben, J. Proteomic analysis of cerebrospinal fluid from multiple sclerosis patients. Proteomics 4, 2117-2124 (2004).

14 Farkas, D. \& Tannenbaum, S. R. Characterization of chemically induced hepatotoxicity in collagen sandwiches of rat hepatocytes. Toxicol Sci 85, 927-934 (2005).

15 Otagiri, M. \& Chuang, V. T. Pharmaceutically important pre- and posttranslational modifications on human serum albumin. Biol Pharm Bull 32, 527-534 (2009).

16 Oettl, K. et al. Oxidative damage of albumin in advanced liver disease. Biochim Biophys Acta 1782, 469-473 (2008)

17 Meucci, E., Mordente, A. \& Martorana, G. E. Metal-catalyzed oxidation of human serum albumin: conformational and functional changes. Implications in protein aging. J Biol Chem 266, 4692-4699 (1991).

18 Taub, R. Liver regeneration: from myth to mechanism. Nat Rev Mol Cell Biol 5, 836-847 (2004).

19 Scharf, J. G. et al. Insulin-like growth factor (IGF)-binding protein-1 is highly induced during acute carbon tetrachloride liver injury and potentiates the IGF-I-stimulated activation of rat hepatic stellate cells. Endocrinology 145, 3463-3472 (2004).

20 Zidek, N., Hellmann, J., Kramer, P. J. \& Hewitt, P. G. Acute hepatotoxicity: a predictive model based on focused illumina microarrays. Toxicological sciences : an official journal of the Society of Toxicology 99, 289-302 (2007).

21 Bhattacharya, S. Mechanisms of signal transduction in the stress response of hepatocytes. Int Rev Cytol 184, 109156 (1998).

22 Hilbrands, L. B., Demacker, P. N., Hoitsma, A. J., Stalenhoef, A. F. \& Koene, R. A. The effects of cyclosporine and prednisone on serum lipid and (apo)lipoprotein levels in renal transplant recipients. J Am Soc Nephrol 5, 2073 2081 (1995).

23 Sposito, A. C. et al. Apolipoprotein and lipid abnormalities in chronic liver failure. Braz J Med Biol Res 30, 12871290 (1997). 
24 Makishima, M. Nuclear receptors as targets for drug development: Regulation of cholesterol and bile acid metabolism by nuclear receptors. J Pharmacol Sci 97, 177-183, (2005).

25 Zollner, G. \& Trauner, M. Mechanisms of cholestasis. Clinics in liver disease 12, 1-26, vii, (2008).

26 Wagner, M., Zollner, G. \& Trauner, M. Nuclear receptor regulation of the adaptive response of bile acid transporters in cholestasis. Seminars in liver disease 30, 160-177, (2010).

27 Loor, F., Tiberghien, F., Wenandy, T., Didier, A. \& Traber, R. Cyclosporins: structure-activity relationships for the inhibition of the human MDR1 P-glycoprotein ABC transporter. J Med Chem 45, 4598-4612 (2002).

28 Kalaany, N. Y. \& Mangelsdorf, D. J. LXRS and FXR: the yin and yang of cholesterol and fat metabolism. Annu Rev Physiol 68, 159-191 (2006).

29 Andersson, M. et al. Modulations in hepatic branch-point enzymes involved in isoprenoid biosynthesis upon dietary and drug treatments of rats. Biochim Biophys Acta 1214, 79-87 (1994).

30 Huang, Z. H. \& Mazzone, T. ApoE-dependent sterol efflux from macrophages is modulated by scavenger receptor class B type I expression. J Lipid Res 43, 375-382 (2002).

31 Shimamura, M. et al. Angiopoietin-like protein 3, a hepatic secretory factor, activates lipolysis in adipocytes. Biochem Biophys Res Commun 301, 604-609 (2003).

32 Liu, J., Afroza, H., Rader, D. J. \& Jin, W. Angiopoietin-like protein 3 inhibits lipoprotein lipase activity through enhancing its cleavage by proprotein convertases. The Journal of biological chemistry 285, 27561-27570 (2010).

33 Amacher, D. E., Adler, R., Herath, A. \& Townsend, R. R. Use of proteomic methods to identify serum biomarkers associated with rat liver toxicity or hypertrophy. Clin Chem $\mathbf{5 1}$, 1796-1803 (2005).

34 Speeckaert, M. M. et al. Investigation of the potential association of vitamin D binding protein with lipoproteins. Ann Clin Biochem 47, 143-150 (2010).

35 Ramagopalan, S. V. et al. A ChIP-seq defined genome-wide map of vitamin D receptor binding: associations with disease and evolution. Genome Res 20, 1352-1360 (2010).

36 Zhang, J. et al. DNA binding alters coactivator interaction surfaces of the intact VDR-RXR complex. Nat Struct Mol Biol 18, 556-563 (2011).

37 Isozaki, M., Ito, K., Masubuchi, Y. \& Horie, T. Plasma retinol binding protein for monitoring the acetaminopheninduced hepatotoxicity. Drug Metab Pharmacokinet 17, 540-545 (2002).

38 Romanowska, A., Lebensztejn, D. M., Skiba, E., Tarasow, E. \& Kaczmarski, M. Retinol binding protein-4 as a serum biomarker of intrahepatic lipid content in obese children--preliminary report. Acta Biochim Pol 58, 35-38 (2011).

39 Stefan, N. et al. High circulating retinol-binding protein 4 is associated with elevated liver fat but not with total, subcutaneous, visceral, or intramyocellular fat in humans. Diabetes Care 30, 1173-1178 (2007). 


\section{CHAPTER 5}

\section{Conserved hepatotoxic responses in HepG2 and primary mouse hepatocytes}

Anke Van Summeren*, Daneida Lizarraga*, Karen J. Brauers, Charly John, Johan Renes, Edwin C. M. Mariman, Jos C. S. Kleinjans and Joost H. M. van Delft

* Both authors contributed equally. 


\section{ABSTRACT}

To identify hepatotoxic compounds out of candidate drugs it is necessary to develop robust in vitro models that enable the reliable prediction of liver toxicity in potentially treated humans. This study aimed to investigate the applicability of primary mouse hepatocytes and human HepG2 cells to detect drug-induced hepatotoxicity. Currently, tests with laboratory animals represent the standard for repeated dose toxicity in man. By comparing primary mouse hepatocytes with HepG2 we aimed to determine whether a particular adverse response observed in a mouse assay is conserved in a human assay.

Previous studies performed with these two in vitro models evaluated only a limited set of genes or proteins. Here we applied a global toxicogenomics approach to compare wholegenome gene expression in both in vitro models after exposure to acetaminophen, cyclosporin $A$ and amiodarone. These compounds were selected based on their specific hepatotoxic phenotypes (necrosis, cholestasis and steatosis). The Metacore ${ }^{\mathrm{TM}}$ toxicity analysis tool was used to determine the specific and conserved 'liver toxicity endpoint processes'. Several hepatotoxic mechanisms were identified. Respective hepatotoxicants appeared to induce similar patterns of gene expression modifications, in particular related to cholesterol metabolism. Furthermore, in combination with a pathway analysis in Tox-profiler this analysis revealed a strong hepatotoxicity-associated conservation at the gene as well as the pathway level among HepG2 and primary mouse hepatocytes. 


\section{INTRODUCTION}

In order to be recognized as safe for humans, new chemical compounds need to be tested on laboratory animals. However, the use of in vivo models in toxicological research is expensive and time-consuming. More importantly, the relevance of outcomes of rodent testing for human health risk seems limited. Furthermore, the use of animals used in toxicity testing is discussed for ethical reasons. Therefore, it is necessary to develop alternative, particular in vitro models, to characterize (hepato-) toxic compounds. Primary human hepatocytes are considered 'the gold standard' among in vitro models for toxicity screening. However, the use of primary human hepatocytes is hindered by the scarcity of suitable liver samples and by interindividual variation due to genetic, life style and age differences of the donors ${ }^{1}$. Primary rat hepatocytes may be used as an alternative for human hepatocytes, although these cells show a relatively rapid decline of their cytochrome P450 (CYP450) enzyme activities with increasing culturing time ${ }^{2}$. Previously it was shown that primary mouse hepatocytes maintain their metabolic competence better compared to primary rat hepatocytes ${ }^{3}$. Moreover, transgenic mouse models are available, which may provide primary hepatocytes suitable for dedicated mechanistic investigations of liver toxicity ${ }^{4}$.

HepG2 are human hepatic carcinoma cells that in contrast to primary hepatocytes are not limited by their availabilty. They are easier to culture, have a longer life span, demonstrate less biological variation and they are widely used in toxicological and toxicogenomics studies $^{5,6}$. Despite the advantages of HepG2, it is fair to note that they have a lower expression of certain phase I drug metabolizing enzymes such as CYP2E1 ${ }^{2,7}$.

Drug-induced hepatotoxicity comprises several phenotypes like necrosis, cholestasis and steatosis. Excessive injury to mitochondria caused by free radicals and/or toxic metabolites may cause necrosis ${ }^{8}$. Cholestasis is induced by impairment of the bile-salt transporter proteins leading to accumulation of bile, causing secondary injury to hepatocytes ${ }^{9}$. Steatosis is caused by a disturbed $\beta$-oxidation of lipids; interruption of the $\beta$-oxidation cycle in hepatocytes may lead to intracellular accumulation of small lipid vesicles ${ }^{10}$.

By comparing primary mouse hepatocytes with HepG2 we aim to determine whether a particular adverse response in a mouse model is conserved in a human model. The extrapolation of adverse responses from a mouse to a human in vitro model is necessary to predict adverse responses in humans.

Furthermore, we analyze whether distinct differences exist between the gene expression profiles of three hepatotoxic model compounds. Therefore, HepG2 and primary mouse hepatocytes were exposed to three well known hepatotoxicants: acetaminophen, amiodarone and cyclosporin A. These compounds are well known for inducing specific liver injury in humans. Acetaminophen is used as an analgesic, which can cause severe liver damage at higher doses and therefore applied as a reference compound for necrosis ${ }^{11}$. Amiodarone, is 
an anti-arrhythmic drug which induce steatosis as adverse side effect ${ }^{10}$. Cyclosporin $A$ is a widely used immunosuppressive drug which has been shown to induce cholestasis ${ }^{12}$. Previously, these compounds were used in both models to examine differentially expressed proteins ${ }^{13,14}$.

\section{MATERIALS AND METHODS}

\section{Chemicals}

Dulbecco's modified Eagle's medium (DMEM), fetal calf serum (FCS), penicillin/streptomycin, Hanks' calcium- and magnesium-free buffer and insulin were obtained from Invitrogen (Breda, The Netherlands). Glucagon, hydrocortisone (water soluble), collagenase type IV, dimethylsulfoxide (DMSO), trypan blue, 3-(4,5-dimethylthiazol-2-yl)-2,5-diphenyltetrazolium bromide, acetaminophen, amiodarone, cyclosporin A (BioChemika) and N,N-dimethylformamide (anhydrous, $99.8 \%$ ) were purchased from Sigma-Aldrich (Zwijndrecht, The Netherlands). Collagen Type I rat tail was obtained from BD BioSciences (Bedford, MA). The Qiazol reagent and RNeasy kit were purchased from Qiagen Westburg bv. (Leusden, the Netherlands).

\section{Animals}

Permission for animal studies was obtained from the Animal Ethical Committee of Maastricht University, the Netherlands (approval no. 2008-075). Adult male C57/B6 mice, weighing 20-25 $\mathrm{g}$, were obtained from Charles River $\mathrm{GmbH}$ (Sulzfeld, Germany). The animals were housed in macrolon cages with sawdust bedding at $22^{\circ} \mathrm{C}$ and $50-60 \%$ humidity. The light cycle was $12 \mathrm{~h}$ light/12 $\mathrm{h}$ dark. Food and tap water were available ad libitum.

\section{Cell culture of primary mouse hepatocytes and HepG2 cells}

The primary mouse hepatocytes were isolated by the two-step collagenase perfusion method according to Seglen ${ }^{15}$, with modifications as described before ${ }^{3}$. Cell suspensions with cell viability $\geq 80 \%$, determined by trypan blue exclusion, were brought into culture in a collagencollagen sandwich formation as described before ${ }^{3}$. The HepG2 cells were cultured as described earlier ${ }^{13}$.

\section{Cytotoxicity Analysis}

The $\mathrm{IC}_{20}$ concentrations of the test compounds (acetaminophen, cyclosporin A or amiodarone) in HepG2 cells and primary mouse hepatocytes were determined after $24 \mathrm{~h}$ exposure using the MTT reduction method ${ }^{16}$. The MTT test for HepG2 was performed under the same conditions as described in ${ }^{13}$, for primary mouse hepatocytes as described before ${ }^{17}$. 


\section{Cell treatment}

The HepG2 cells and primary mouse hepatocytes were exposed to the $\mathrm{IC}_{20}$ concentrations determined after an exposure time of 24 hours. The HepG2 cells were exposed to $10 \mathrm{mM}$ acetaminophen, $50 \mu \mathrm{M}$ cyclosporin A and $25 \mu \mathrm{M}$ amiodarone for $24,48 \mathrm{~h}$ and $72 \mathrm{~h}$. The primary mouse hepatocytes were exposed to $10 \mathrm{mM}$ acetaminophen, $15 \mu \mathrm{M}$ amiodarone or $20 \mu \mathrm{M}$ cyclosporin A for 24 and $48 \mathrm{~h}$. Time points later than $24 \mathrm{~h}$ at $\mathrm{IC}_{20}-24 \mathrm{~h}$ were analyzed in order to extend the observed damage by the compounds in this toxicity analysis. For HepG2 a $72 \mathrm{~h}$ time point was included, which make it possible to compare the mRNA expression with the previously presented protein expression after $72 \mathrm{~h}$ exposure ${ }^{13}$. All compounds were dissolved in DMSO and added to the medium with a final concentration of $0.5 \% \mathrm{v} / \mathrm{v}$ DMSO. Cells incubated in the presence of $0.5 \% \mathrm{v} / \mathrm{v}$ DMSO served as control. The cells were obtained from three independent biological experiments (each from a different animal).

\section{Total RNA isolation and microarray experiments}

Total RNA was isolated after 24 and $48 \mathrm{~h}$ of incubation with acetaminophen, amiodarone, cyclosporin A or DMSO in HepG2 and primary mouse hepatocytes. Total RNA was isolated from cells using the miRNeasy mini Kit (Qiagen Westburg, Leusden, the Netherlands) according to the manufacturer's instructions and followed by a DNAse I (Qiagen Westburg) treatment. RNA quantity was measured on a spectrophotometer and quality was determined on a BioAnalyzer (Agilent Technologies, Breda, the Netherlands). Only RNA samples that showed clear $18 \mathrm{~S}$ and $28 \mathrm{~S}$ peaks and obtained a RIN level higher than 8 were used. Sample preparation, hybridization, washing, staining and scanning of the Affymetrix Human Genome U133 Plus 2.0 GeneChip arrays were conducted according to the manufacturer's instructions as previously described ${ }^{18}$. Quality controls were within acceptable limits. Hybridization controls were present on all arrays and yielded the expected increases in intensities.

\section{Gene expression analyses}

The Affymetrix .CEL files were imported in R 2.11. by using the Affymetrix library in BioConductor ${ }^{19}$. All probes were reannotated using the BrainArray custom CDF files (EntrezGene, version 11.0.1), followed by a RMA normalization step. Prior to the statistical analysis all probe sets (excluding positive and negative controls spotted in the array) had to pass a detection filter criterium [Present/Marginal $\geq 2$ out of 3 biological replicates per condition, control or treated], resulting in a total of 12,518 reporters in HepG2 and 10,848 reporters in primary mouse hepatocytes. One-way ANOVA was used to calculate $p$-values per compound, differentially expressed genes (DEGs) with a $|F C| \geq 1.5$ and $p$-value $<0.05$ for at least one point were considered as significant. To compare the gene expression results in HepG2 and primary mouse hepatocytes, the Orthologene Library in ArrayTrack ${ }^{\mathrm{TM}}{ }^{20}$ (version 3.4.5., U.S. Food and Drug Administration, Silver Spring, USA) was used for obtaining comparable IDs for the human and mouse arrays. From the Orthologene function, the human IDs used for further analyses 
were the human EntrezGene IDs. DEGs conserved between models and compounds, were selected with at least two of the three compounds per in vitro model. Hierarchical clustering was done with Arraytrack $^{\mathrm{TM}}$ (version 3.4.5., U.S. Food and Drug Administration, Silver Spring, USA).

\section{Pathway analysis by MetaCore.}

The MetaCore ${ }^{\mathrm{TM}}$ software (GeneGo, San Diego, USA) was used for biological interpretation of the modulated mRNAs after acetaminophen, amiodarone and cyclosporin A exposure per time point, thereby focusing on the GeneGo 'Liver toxicity endpoint processes'. Experimental data is illustrated and rank-based in fold changes and $p$-values in MetaCore ${ }^{\mathrm{TM}}(|\mathrm{FC}| \geq 1.5$ and p-value $<0.05)$. In case of 'Liver toxicity endpoint processes', their $p$-values were calculated based on the targets that were present in the data per time point and per 'Liver toxicity endpoint processes'. Representative 'Liver toxicity endpoint processes' that showed pvalues $<0.05$ were considered as significant in our data.

MetaCore $^{\mathrm{TM}}$ identifies and visualizes the involvement of DEGs in specific cellular pathways and processes. In the present study we performed a toxicity analysis, which is a new feature in MetaCore $^{\mathrm{TM}}$, by which the toxicogenomic data was assembled in organ-specific toxicity ontologies. The ontologies we have investigated, were liver specific. These 'organ-centered' functional ontologies contain groups of network objects (genes/proteins) relevant to the analysis of organ-specific gene expression and proteomics data. By design, this is a mixed ontology that combines normal and pathological processes with organ-specific gene markers. For instance, the liver ontology includes genes known to contribute to the development of cholestasis and fibrosis, as well as to normal cellular processes such as cell cycle and apoptosis, which, when perturbed, may lead to a pathology. Also included are lists of drug metabolizing enzymes and targets of nuclear receptors controlling the expression of metabolic enzymes. In all cases, the lists are comprised of genes from pathways implicated in the different aspects of the organ's functionality. Organ-specific toxicity map ontologies have also been compiled by GeneGo experts. These are collections of organ-specific pre-built maps ${ }^{21}$. During the analysis the DEGs from both in vitro models were compared. After that the top 10 enriched toxicity processes for (A) common DEGs and (B) similar DEGs and (C) DEGs unique to each treatments or in vitro model were identified. Common DEGs is the set of genes that are present in all of the active experiments, while similar DEGs is the set of genes that are present in more than one active experiment, but not in all of them. During the analysis, a background list was used. The background list used contained 13893 genes that resulted from the data filtering used from both in vitro liver models.

\section{Pathways analysis by ToxProfiler.}

The complete data set of genes was used as input data into the analysis without statistically pre-selecting any DEGs. ToxProfiler uses the t-test to score the difference between the mean 
expression level of predefined groups of genes and that of all other genes within the complete data set of genes (http://www.t-profiler.org) ${ }^{22,23}$. This is in contrast with MetaCore ${ }^{\mathrm{TM}}$ analysis which requires such a pre-selection. In the present study, the predefined gene groups are based on specific GoProcesses or from part of a pathway defined by the KEGG.
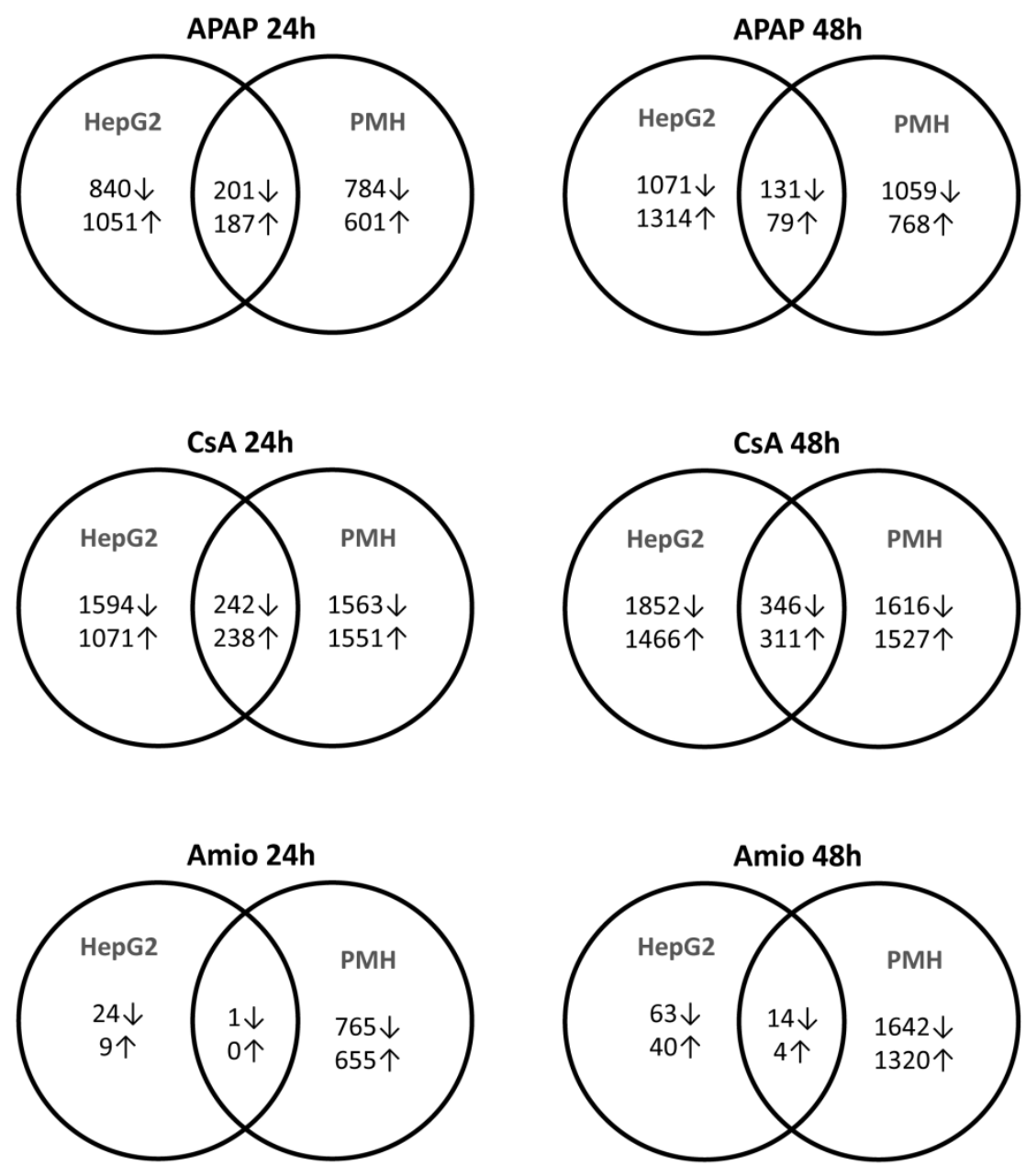

Figure 5.1: Venn diagrams with DEGs between HepG2 cells and primary mouse hepatocytes (PMH) cells after acetaminophen (APAP), cyclosporin A (CSA) and amiodarone (Amio) exposure. 


\section{RESULTS}

\section{Differentially expressed genes}

In order to identify specific and conserved gene expression changes both HepG2 and primary mouse hepatocytes were exposed for 24 and $48 \mathrm{~h}$ to acetaminophen, amiodarone, cyclosporin A or DMSO. In case of HepG2 cells, a $72 \mathrm{~h}$ time point was also included so the mRNA expression can be compared with the previously presented protein expression after $72 \mathrm{~h}$ exposure ${ }^{13}$. Only one dose was investigated at multiple time points, since it was shown previously that in in vitro models treatment time has a larger impact on chemically induced gene expression than the dose ${ }^{17,24,25}$. Expression changes in both in vitro models at its $\mathrm{IC}_{20}-24 \mathrm{~h}$ over time were identified by Affymetrix arrays. From the 16,395 genes in Affymetrix arrays, 12,518 genes could be analyzed for HepG2 and 10,848 genes (after orthologene 10,002 genes) for primary mouse hepatocytes. One-way ANOVA ( $p$-value $<0.05$ ) combined with an absolute fold change $\geq 1.5$ was used to identify significant DEGs per time point for each compound. The amount of DEGs of in HepG2 and primary mouse hepatocytes after exposure of the three compounds is presented in Supplemental Table 5.1.

The overlap of DEGs between HepG2 and primary mouse hepatocytes exposed to acetaminophen, cyclosporin A and amiodarone is shown in Figure 5.1. The overlap is the highest for cyclosporin $A$ and the lowest for amiodarone, this probably because amiodarone induced a relatively small effect at gene expression level in HepG2.

The Hierarchical clustering performed with Arraytrack ${ }^{\mathrm{TM}}$ revealed a proper cluster of the three compounds based on the gene expression in HepG2 (Supplemental Figure 5.1), which was in contrast to the clustering based on the gene expression in primary mouse hepatocytes.

\section{Pathway analysis by MetaCore ${ }^{T M}$}

MetaCore ${ }^{\mathrm{TM}}$ was used to analyze the pathways involved the liver toxicity, induced by each compound at a specific time point and to detect the common and similar processes affected across time. In the MetaCore ${ }^{\mathrm{TM}}$ pathway analysis, the $p$-values of the 'Liver toxicity endpoint processes' were calculated based on the targets that were present in the 'common' and 'similar' data between the models. In Figure 5.2 the top 10 enriched 'Liver toxicity endpoint processes' after acetaminophen exposure in HepG2 and primary mouse hepatocytes are shown.

In the liver acetaminophen is transformed to the reactive metabolite NAPQI which depletes glutathione by conjugation reaction. The loss of glutathione will lead to a decreased detoxification of reactive oxygen species (ROS) and thereby increasing oxidative stress. ROS can cause severe injury to the mitochondria which eventually will lead to necrosis ${ }^{26}$. In the top 10 of the 'Liver toxicity endpoint processes', development of oxidative stress was found at the fifth place and mainly detected in primary mouse hepatocytes exposed for $48 \mathrm{~h}$, with a $-\log (\mathrm{p}-$ value) of 2 (0.009385 in linear scale) (Figure 5.2). 
Top 10 enriched GeneGo Liver toxicity endpoint processes after APAP exposure

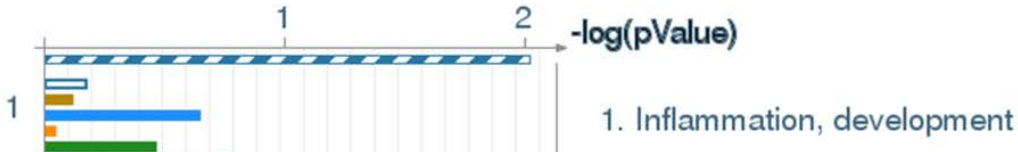

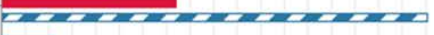
2. Steatosis, development_liver
2

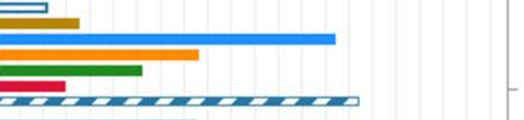
3

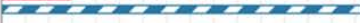

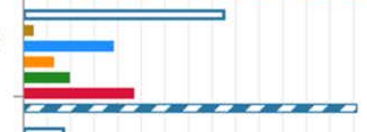
4

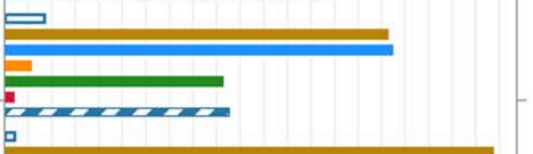
5

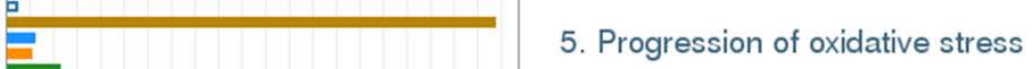
3. Cholestasis, development_liver
4. Peroxisomal proliferation, induction liver
6 arian

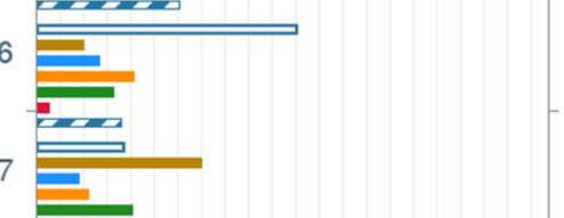
6. Hypertrophic organ growth_liver
8

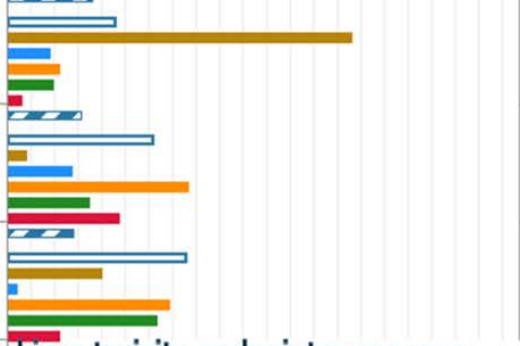
7. Fibrosis, development_liver
8. Ischemia-induced cellular changes_ liver
9. Phospholipidosis, development_liver
10. Cell cycle, processes involved in G0- phase
Liver toxicity endpoint processes

$$
\begin{aligned}
& \text { Legend } \\
& \begin{array}{|l|l}
\hline \text { APAP HepG2 } 24 \text { hour } \\
\text { APAP HepG2 } 48 \text { hour } \\
\text { APAP HepG2 } 72 \text { hour } \\
\text { APAP PMH } 24 \text { hour } \\
\text { APAP PMH } 48 \text { hour }
\end{array}
\end{aligned}
$$

\section{Common}

Figure 5.2: Top 10 enriched 'Liver toxicity endpoint processes' after acetaminophen (APAP) exposure in HepG2 and primary mouse hepatocytes (PMH). Sorting is done for the 'common' set of genes between the different time points and in vitro liver models. $X$-axis values are a negative $\log 10$ transformation of the $p$-value from a hypergeometric distribution. Colors and patterns of the bars represent the different DEG sets. 
Top 10 enriched GeneGo Liver toxicity endpoint processes after CsA exposure

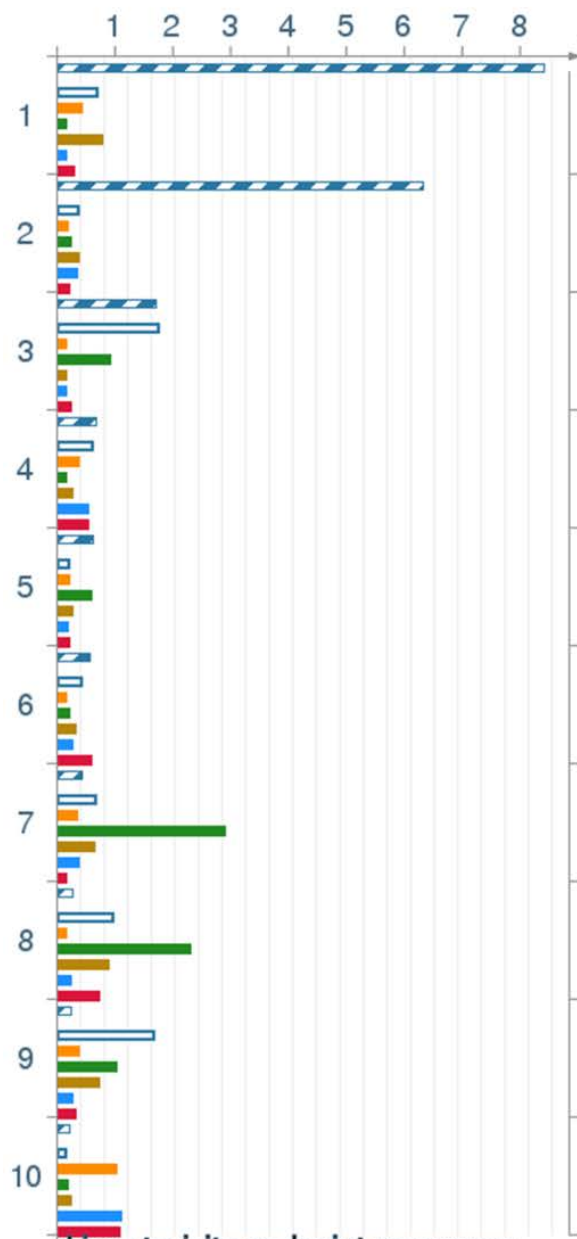

\section{$-\log (p$ Value $)$}

1. Steatosis, development_liver

2. Peroxisomal proliferation, induction liver

3. Cholestasis, development_liver

4. Hypertrophic organ growth_liver

5. Progression of oxidative stress

6. Inflammation, development

7. Ischemia-induced cellular changes liver

8. Apoptosis via Mitochondrial membrane dysfunction

9. Phospholipidosis, development_liver

10. Cell cycle, processes involved in G1phase

\begin{tabular}{|c|c|}
\hline Legend & \\
\hline CsA Hepg2 24 hour & Common \\
\hline CsA HepG2 48 hour & Similar \\
\hline CsA HepG2 72 hour & \\
\hline CsA PMH 24 hour & \\
\hline CsA PMH 48 hour & \\
\hline
\end{tabular}

Figure 5.3: Top 10 enriched 'Liver toxicity endpoint processes' after cyclosporin A (CsA) exposure in HepG2 and primary mouse hepatocytes (PMH). Sorting is done for the 'common' set of genes between the different time points and in vitro liver models. $\mathrm{X}$-axis values are a negative $\log 10$ transformation of the $\mathrm{p}$-value from a hypergeometric distribution. Colors and patterns of the bars represent the different DEG sets. 
Top 10 enriched GeneGo Liver toxicity endpoint processes after Amio exposure

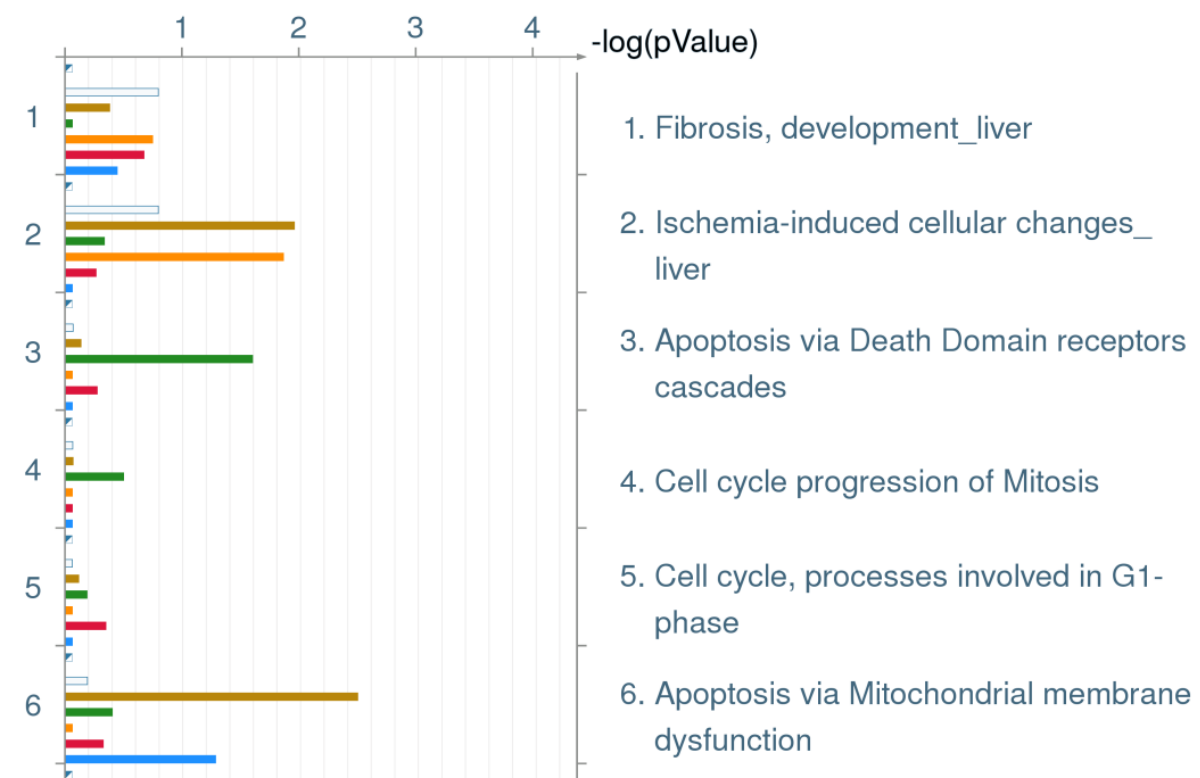

7. Necrosis, development

8. Cholestasis, development_liver

9. Progression of oxidative stress

10. Cell cycle, processes involved in G0phase

Liver toxicity endpoint processes

\begin{tabular}{|l|l|l|}
\hline Legend \\
\hline Amio HepG2 24 hour \\
Amio HepG2 48 hour \\
Amio HepG2 72 hour \\
Amio PMH 24 hour \\
Amio PMH 48 hour
\end{tabular}

Figure 5.4: Top 10 enriched 'Liver toxicity endpoint processes' after amiodarone (Amio) exposure in HepG2 and primary mouse hepatocytes (PMH). Sorting is done for the 'common' set of genes between the different time points and in vitro liver models. $X$-axis values are a negative $\log 10$ transformation of the $p$-value from a hypergeometric distribution. Colors and patterns of the bars represent the different DEG sets. 
For acetaminophen the top 3 of the common 'Liver toxicity endpoint processes' between HepG2 and primary mouse hepatocytes are development of inflammation, steatosis and cholestasis. For cyclosporin A the top 10 enriched 'Liver toxicity endpoint processes' in HepG2 and primary mouse hepatocytes are shown in Figure 5.3. Cyclosporin $A$ is a strong immunosuppressant and induces cholestasis as adverse reaction ${ }^{27}$. In the top 10 of the 'Liver toxicity endpoint processes', the development of cholestasis was found at the third place mainly due to common regulated gene expressions between HepG2 and primary mouse hepatocytes.

Other processes in the top 3 of the common 'Liver toxicity endpoint processes' between HepG2 and primary mouse hepatocytes are steatosis and peroxisomal proliferation. Figure 5.4 shows the top 10 enriched 'Liver toxicity endpoint processes' after amiodarone exposure in HepG2 and primary mouse hepatocytes. Amiodarone is used as an anti-arrhythmic drug and has hepatic steatosis as a toxic side effect due to an inhibited $\beta$-oxidation of fatty acids ${ }^{10}$. However, development of steatosis appeared not to be present in this top 10 of enriched 'Liver toxicity endpoint processes'. The only similar process detected after amiodarone exposure in HepG2 and primary mouse hepatocytes was cholestasis (with a -log(p-value) of 4 (0.00006857 in linear scale) (Figure 5.4). The pathway analysis with Metacore ${ }^{\mathrm{TM}}$ revealed some specific and conserved hepatotoxic pathways like cholestasis, inflammation and steatosis in HepG2 and primary mouse hepatocytes.

\section{KEGG Pathway analyses by ToxProfiler}

Based on gene expression data from HepG2 and primary mouse hepatocytes after acetaminophen, cyclosporin A and amiodarone exposure, ToxProfiler identified several biochemical/cellular pathways and biological processes, enabling a comparison between HepG2 and primary mouse hepatocytes at molecular level. Furthermore, this analysis showed the direction in which a pathway is regulated by each compound (up/down-regulated), This information provides a better insight in the most conserved pathways between both in vitro models.

Figure 5.5 shows the number of KEGG pathways in HepG2 and primary mouse hepatocytes modified by at least one of the compounds and the overlap of KEGG pathways as identified with Tox profiler.

With ToxProfiler 23 KEGG pathways were identified in primary mouse hepatocytes exposed to acetaminophen, while 7 KEGG pathways were identified in HepG2. These 7 pathways were all conserved in primary mouse hepatocytes and included glycolysis/gluconeogenesis, primary bile acid biosynthesis, glycine, serine and threonine metabolism, chromosome, transporters, complement and coagulation cascades, PPAR signaling pathway (Figure 5.5). Based on the GO Processes 21 processes were identified in primary mouse hepatocytes and only 1 (extracellular space) in HepG2 which was conserved in primary mouse hepatocytes (Supplemental table 5.2 and Supplemental Figure 5.2). 
For cyclosporin A, 19 KEGG pathways were detected in HepG2, from which 3 were also identified in primary mouse hepatocytes, comprising glycolysis/gluconeogenesis, fatty acid metabolism and complement and coagulation cascades (Figure 5.5). Notably, HepG2 showed more significant pathways modulated by cyclosporin A in comparison to primary mouse hepatocytes. Cyclosporin A treatment resulted in 23 modified GO Processes in HepG2 from which Lipid transporter activity, Extracellular space and very-low-density lipoprotein particle were conserved in primary mouse hepatocytes (Supplemental table 5.2 and Supplemental Figure 5.2).

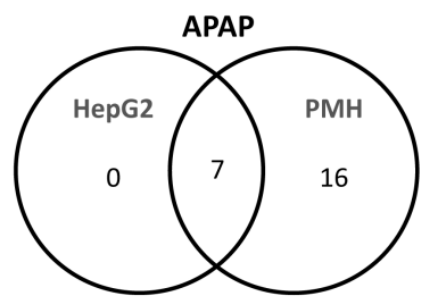

1. Glycolysis / Gluconeogenesis

2. Primary bile acid biosynthesis

3. Glycine, serine and threonine metabolism

4. Chromosome

5. Transporters

6. Complement and coagulation cascades

7. PPAR signaling pathway

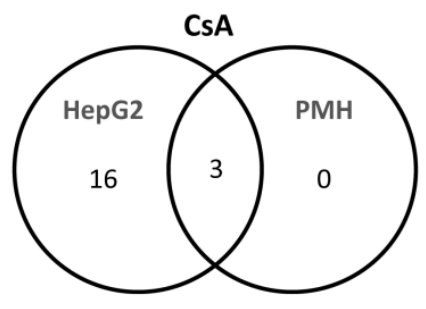

1. Glycolysis / Gluconeogenesis

2. Fatty acid metabolism

3. Complement and coagulation cascades

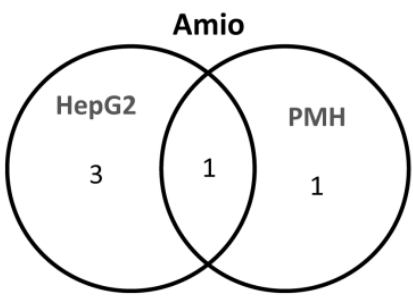

1. Complement and coagulation cascades

Figure 5.5: Venn diagrams with overlapped significant KEGG pathways after acetaminophen, cyclosporin A and amiodarone exposure in HepG2 and primary mouse hepatocytes (PMH).

According to the ToxProfiler analysis, amiodarone modified 4 KEGG pathways in HepG2 and 2 in primary mouse hepatocytes from which the pathway complement and coagulation cascades was conserved in both models (Figure 5.5). Amiodarone altered $3 \mathrm{GO}$ Processes in HepG2 and 1 in primary mouse hepatocytes, but no processes were conserved (Supplemental Figure 5.2). The reduced overlap between HepG2 and primary mouse hepatocytes regarding amiodarone is due to the poor number of DEGs after amiodarone exposure. Table 5.1 shows the conserved KEGG pathways per compound and demonstrates that those are significantly modulated in the same direction in both HepG2 and primary mouse hepatocytes.

As determined by ToxProfiler, the hepatotoxicants induced 11 common KEGG pathways in HepG2 and primary mouse hepatocytes, shown in Table 5.1. The applied hepatotoxicants modified mainly major liver functions like glycolysis/gluconeogenesis; primary bile acid biosynthesis, fatty acid metabolism, steroid hormone biosynthesis and drug metabolism. 
Table 5.1: Conserved KEGG Pathways in both in vitro models (HepG2 cells and primary mouse hepatocytes) after the exposure to acetaminophen (APAP), amiodarone (Amio) and cyclosporin $\mathrm{A}$ (CSA) per time point.

\begin{tabular}{|c|c|c|c|c|c|c|c|c|c|c|c|c|c|c|c|}
\hline \multirow[t]{3}{*}{ KEGG Pathways } & \multicolumn{9}{|c|}{ HepG2 cells (t-values) } & \multicolumn{6}{|c|}{ primary mouse hepatocytes (t-values) } \\
\hline & APAP & APAP & APAP & Amio & Amio & Amio & CsA & CsA & CsA & APAP & APAP & Amio & Amio & CsA & CsA \\
\hline & $24 \mathrm{~h}$ & $48 \mathrm{~h}$ & $72 \mathrm{~h}$ & $24 \mathrm{~h}$ & $48 \mathrm{~h}$ & $72 \mathrm{~h}$ & $24 \mathrm{~h}$ & $48 \mathrm{~h}$ & $72 \mathrm{~h}$ & $24 \mathrm{~h}$ & $48 \mathrm{~h}$ & $24 \mathrm{~h}$ & $48 \mathrm{~h}$ & $24 \mathrm{~h}$ & $48 \mathrm{~h}$ \\
\hline $\begin{array}{l}\text { Glycolysis / Glucone- } \\
\text { ogenesis }\end{array}$ & -5.79 & -4.53 & -2.45 & -0.13 & 2.02 & -0.51 & -10.16 & -5.26 & -4.79 & -4.38 & -1.94 & -2.43 & -3.23 & -3.77 & -4.12 \\
\hline $\begin{array}{l}\text { Fatty acid metabo- } \\
\text { lism }\end{array}$ & -3.28 & -3.35 & -2.19 & -1.45 & -2.42 & -3.29 & -14.23 & -5.87 & -6.36 & -2.89 & -0.25 & -3.79 & -4.45 & -5.55 & -5.05 \\
\hline $\begin{array}{l}\text { Primary bile acid } \\
\text { biosynthesis }\end{array}$ & -3.96 & -4.92 & -3.83 & -0.05 & -1.2 & -2.08 & -2.65 & -3.78 & -3.79 & -5.39 & 0.3 & 0.76 & 1.33 & -0.77 & -0.61 \\
\hline $\begin{array}{l}\text { Glycine, serine and } \\
\text { threonine metabo- } \\
\text { lism }\end{array}$ & -2.9 & -4.93 & -2.92 & 0.19 & -2.79 & -3.57 & -5.08 & -5.62 & -5.05 & -4.02 & 0.51 & -0.56 & 0.14 & 0.03 & -0.27 \\
\hline Chromosome & -1.49 & 5.92 & 7.57 & -3.11 & 2.39 & -4.48 & -4.24 & 1.45 & 1.41 & 4.88 & 1.01 & 0.82 & 0.95 & 0.32 & 0.11 \\
\hline Transporters & -2.53 & -4.6 & -3.53 & -0.87 & -0.79 & 1.22 & -2.8 & -3.09 & -3.68 & -5.74 & 0.42 & -2.38 & -1.26 & -2.57 & -1.24 \\
\hline $\begin{array}{l}\text { Complement and } \\
\text { coagulation cascades }\end{array}$ & -6.32 & -7.31 & -6.31 & -1.81 & -3 & -6.37 & -10.46 & -7.21 & -10.97 & -6.78 & -3.22 & -3.15 & -4.25 & -4.96 & -5.58 \\
\hline $\begin{array}{l}\text { PPAR signaling } \\
\text { pathway }\end{array}$ & -4.07 & -5.08 & -3.14 & -1.63 & -1.38 & -1.96 & -7.5 & -6.71 & -6.94 & -4.59 & -2 & -2.47 & -3.38 & -3.24 & -3.84 \\
\hline
\end{tabular}

Positive and negative t-values indicate predominant up- and down-regulation of genes within the term. All the significant KEGG pathways highlighted in this table have an evalue (Bonferroni corrected $p$-value (24)) below 0.05 and $\mid t$-value $\mid \geq 4$. 


\section{DISCUSSION}

In the present study a mouse in vitro model (primary mouse hepatocytes) and a human in vitro model (HepG2) were compared to detect hepatotoxic response pathways conserved across species. In addition, we examined whether distinct differences exist between the gene expression levels of three model compounds. HepG2 and primary mouse hepatocytes were exposed to acetaminophen, cyclosporin $\mathrm{A}$ and amiodarone and the effects of these compounds were measured by a large-scale transcriptome analysis. Each compound represents another class of liver injury, e.g. either necrosis (acetaminophen), cholestasis (cyclosporin A) or steatosis (amiodarone).

Both in vitro models were exposed for $24 \mathrm{~h}$ and $48 \mathrm{~h}$ to acetaminophen, cyclosporin $\mathrm{A}$, amiodarone or to $0.5 \%$ DMSO as vehicle control. In case of HepG2 cells, a $72 \mathrm{~h}$ time point was also included in the study. When evaluating numbers of DEGs in HepG2 as well as in primary mouse hepatocytes, cyclosporin A appeared to induce the most significant effects (Figure 5.1). This is in agreement with previously detected protein expression in these models after exposure to the same test compounds ${ }^{13,28}$. Acetaminophen as well as amiodarone induced a relatively small effect on the proteome ${ }^{13,28}$, while cyclosporin A treatment resulted in remarkable proteome changes in both models ${ }^{13,28}$.

At the mRNA level amiodarone induced a relatively small effect in HepG2 cells (Figure 5.1). It is known that the HepG2 cells have partially lost some of the liver specific functions due to immortalization, in particular the phase I drug metabolizing enzymes ${ }^{2,7}$. Nevertheless, the biotransformation capacity of HepG2 has been extensively reported ${ }^{6,29}$. Amiodarone is an indirect toxin and requires biotranformation for toxicity. Amiodarone is extensively metabolized in the liver by CYP3A4 to its toxic mono-N-desethyl and di-N-desethyl metabolites ${ }^{30}$. Previously it was shown that amiodarone was less toxic in wild type HepG2 than in HepG2 cells with overexpression of CYP3A4 ${ }^{30}$. A low expression of CYP3A4 leads to lower levels of toxic metabolites so only a relatively small effect on the transcriptome can be observed. Furthermore, a hierarchical clustering was performed using the generated list with DEGs (Supplemental Figure 5.1). In contrast to primary mouse hepatocytes, gene expression data in HepG2 allowed us to make a proper cluster of the three compounds. Despite the fact that the three compounds induce different endpoints, in primary mouse hepatocytes it appeared not possible to generate a proper distinction between compounds. However, it should be mentioned that drugs inducing different endpoints, may still trigger similar mechanisms of action. Primary mouse hepatocytes have a stable gene expression of phase I and phase II enzymes ${ }^{3}$. Furthermore, they are better metabolically competent than HepG2 cells. Therefore, primary mouse hepatocytes are capable of activating multiple mechanisms, which thus may complicate a clustering analysis.

In our study we first used the MetaCore ${ }^{\mathrm{TM}}$ toxicity analysis to determine the specific and conserved 'Liver toxicity endpoint processes' for HepG2 and primary mouse hepatocytes exposed 
to acetaminophen, amiodarone and cyclosporin A. Based on the MetaCore ${ }^{\mathrm{TM}}$ toxicity analysis the hepatotoxic phenotypes induced by the compounds were verified. In the common pathways between HepG2 and primary mouse hepatocytes the development of inflammation was enriched in the GeneGo toxicity endpoint processes after exposure to the necrotic compound acetaminophen. In contrast to apoptosis, necrosis is traditionally associated with inflammation ${ }^{31}$. Furthermore, acetaminophen also enriched the progression of oxidative stress endpoint process. It is known that acetaminophen-induced necrosis is linked to increased oxidative stress due to loss of glutathione which induces a decreased ROS detoxification ${ }^{26}$. However, results obtained from the toxicity analysis could not distinguish between the specific hepatotoxic effects per compound. By contrast, cholestasis was not only significant in the 'common' and 'similar' genes after the exposure of cyclosporin A, the model compound for cholestasis, but was also significant in the 'common' and 'similar' genes after acetaminophen and amiodarone exposure. In particular cholesterol metabolism appeared to be commonly deregulated by these three hepatotoxicants. Cholestasis is the accumulation of bile in the liver, which is accompanied by increased serum levels of bile, bilirubin and LDL cholesterol. Consequently, the modulated expression of genes involved in cholesterol and bile acid metabolism are linked to cholestasis. This was the reason to depict commonly expressed cholestasisrelated genes in a network presented in Figure 5.6. This network shows the down-regulation of CYP7A1, this indicates an adaptive response to cyclosporin A-induced cholestasis. CYP7A1 is in fact responsible for the initiation of the bile acid biosynthetic pathway through the conversion of cholesterol to bile acids ${ }^{32}$. Consequently, a down-regulation of CYP7A1 will lead to a decrease of the continuous bile acid production.

This is probably an adaptive response to the increasing cellular bile concentration, due to the inhibition of the bile transport proteins by cyclosporin $A^{33}$. In addition, CYP7B1 is responsible for the conversion of cholesterol to oxysterols before further metabolism to bile acids ${ }^{32}$. Down-regulation of mRNA of this enzyme is also an adaptive response to cyclosporin Ainduced cholestasis. However, besides cyclosporin A, acetaminophen as well as amiodarone induce a decreased expression of CYP7A1 and CYP7B1. Instead of CYP7A1 and CYPB1, the down-regulation of CYP51 is exclusively mediated by cyclosporin A and conserved in both models. Previously it was shown that the cholesterol biosynthesis is regulated by silencing of CYP51 and squalene synthase via the liver $X$ receptor $\alpha^{34}$. Probably the down-regulation of CYP51 is a hepaprotective response specific for drug-induced cholestasis and conserved in primary mouse hepatocytes. 


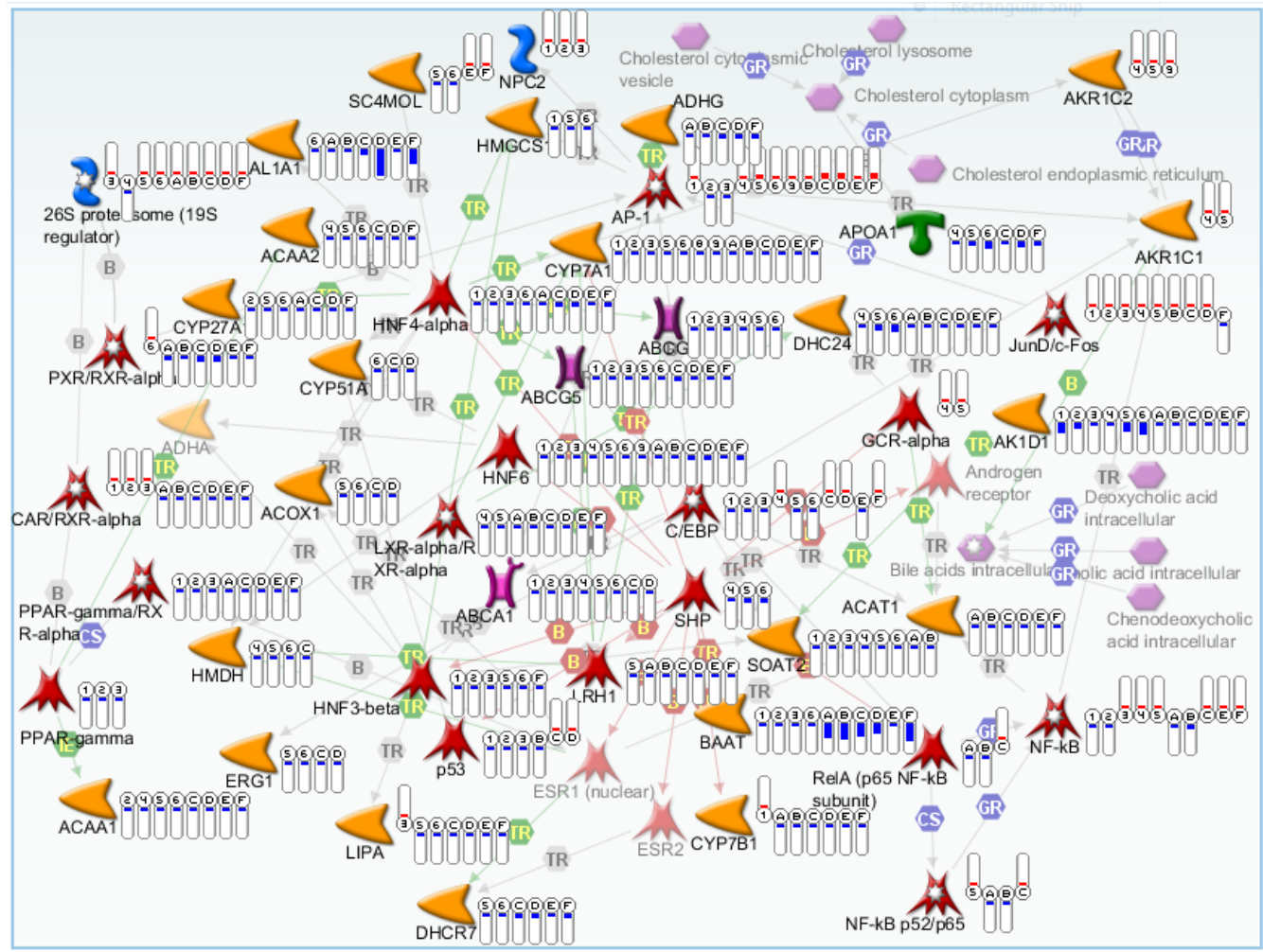

Figure 5.6: Cholestasis network in HepG2 cells and primary mouse hepatocytes after exposure to acetaminophen (APAP), cyclosporin A (CSA) and amiodarone (Amio) across time. Cholestasis was a GeneGO Liver toxicity endpoint process that was significantly altered in HepG2 cells and primary mouse hepatocytes after treatment with acetaminophen, amiodarone and cyclosporin $\mathrm{A}$. The red and blue thermometers denote up-regulation and downregulation, respectively. The thermometer numbering corresponds to HepG2: (1) APAP_24h, (2) APAP_48h, (3) APAP_72h, (4) CsA_24h, (5) CsA_48h and (6) CsA_72h, (7) Amio_24h, (8) Amio_48h, (9) Amio_72h of exposure; and to primary mouse hepatocytes: (A) APAP_24h, (B) APAP_48h, (C) CsA_24h, (D) CsA_48h, (E) Amio_24h and (F) Amio_48h of exposure. The green and red lines between elements denote stimulation and inhibition, respectively. The gray arrows denote unknown interaction between the elements. Significantly up-regulated or down-regulated genes: $F C \geq 1.5$ ( $p$-value $<0.05$ ) or FC $\leq-1.5$ ( $p$-value $<0.05$ ), respectively. The boxes on the lines denote the type of regulation: $M=$ microRNA binding; $T R=$ transcriptional regulation; $+P=$ phosphorylation; $B=$ binding; $C S=c o m p l e x$ subunit; $\mathrm{GR}=$ group relation.

ToxProfiler was used to further identify possibly conserved responses between the HepG2 cells and primary mouse hepatocytes. Acetaminophen induced in both cell types biochemical pathways related to major liver functions. These are glycolysis/gluconeogenesis, primary bile acid biosynthesis (down-regulated in agreement with the changes related with cholestasis at pathway (MetaCore ${ }^{\mathrm{TM}}$ ) and gene level), glycine, serine and threonine metabolism, chromosome, transporters, complement and coagulation cascades and PPAR signaling pathway. These conserved acetaminophen-induced pathways are all down-regulated, except for chromosome-related pathways, Previously, it was also shown that acetaminophen modulates biochemical pathways and processes related to major liver functions such as fatty acid me- 
tabolism, bile acid synthesis, glycolysis/gluconeogenesis, amino acid metabolism and metabolism of xenobiotics by CYP450 ${ }^{23}$. Acetaminophen-induced necrosis is associated with a decreased mitochondrial function and alterations in energy homeostasis ${ }^{35}$. These alterations are characterized primarily by a decreased production of ATP by mitochondria ${ }^{35}$. A decreased ATP production will lead to a repression of energy-consuming pathways like primary bile acid biosynthesis, glycine, serine and threonine metabolism and transporters. Furthermore, the glycolysis/gluconeogenis and PPAR signaling pathway are important regulators of energy homeostasis. The down-regulation of these biochemical pathways are important mechanisms in acetaminophen-induced necrosis which are conserved in both in vitro models.

Primary mouse hepatocytes and HepG2 share the following cyclosporin A-modulated pathways: glycolysis / gluconeogenesis, fatty acid metabolism and complement and coagulation cascades, which were all down-regulated. Previously it was shown that cholestasis is linked with an impaired fatty acid metabolism due to a decreased ketogenesis ${ }^{36}$. Probably in cyclosporin A-induced cholestasis the fatty acids are esterified and exported as very low-density lipoproteins ${ }^{36}$, which might explains abnormal lipoprotein plasma levels of cyclosporine A treated patients. ${ }^{37}$. Therefore the preservation of the fatty acid metabolism is an important mechanism for cyclosporine A-induced cholestasis. The primary bile acid metabolism is a key mechanism in the development of cholestasis. Although not significant we did observe a down regulation of the cholestasis pathway in both models.

Complement and coagulation cascades was the only conserved pathway for amiodarone. The reduced overlap between HepG2 and primary mouse hepatocytes regarding amiodarone is due to the poor number of DEGs after amiodarone exposure. For all three compounds Complement and coagulation cascades appeared a conserved response between HepG2 and primary mouse hepatocytes. The modulation of Complement and coagulation cascades indicates cellular damage and an acute phase response. Acute phase is a clinical state that occurs after many inflammatory stimuli, including xenobiotic stimuli.

In conclusion, with use of the MetaCore ${ }^{\mathrm{TM}}$ toxicity analysis we identified several hepatotoxic mechanisms induced by the three investigated compounds. Respective hepatotoxicants appeared to induce similar patterns of gene expression modifications, in particular related to cholesterol metabolism. Furthermore, pathway analysis of the transcriptomic data reveals hepatotoxicity-associated conserved responses among HepG2 and primary mouse hepatocytes. These conserved responses indicate that adverse reactions in primary mouse hepatocytes have the possibility to predict hepatotoxicty in humans. 


\section{REFERENCES}

1 Donato, M. T., Lahoz, A., Castell, J. V. \& Gomez-Lechon, M. J. Cell lines: a tool for in vitro drug metabolism studies. Curr Drug Metab 9, 1-11 (2008).

2 Boess, F. et al. Gene expression in two hepatic cell lines, cultured primary hepatocytes, and liver slices compared to the in vivo liver gene expression in rats: possible implications for toxicogenomics use of in vitro systems. Toxicol Sci 73, 386-402 (2003).

3 Mathijs, K. et al. Assessing the metabolic competence of sandwich-cultured mouse primary hepatocytes. Drug Metab Dispos 37, 1305-1311 (2009).

4 van Kesteren, P. C. et al. Deregulation of cancer-related pathways in primary hepatocytes derived from DNA repair-deficient Xpa-/-p53+/- mice upon exposure to benzo[a]pyrene. Toxicol Sci 123, 123-132 (2011).

5 Van Summeren, A., Renes, J., Mariman, E. C., Kleinjans, J. C. \& van Delft, J. H. Response to Pathophysiological Relevance of Proteomics Investigations of Drug-Induced Hepatotoxicity in HepG2 Cells. Toxicol Sci 121, 431-433 (2011).

6 Knasmuller, S. et al. Use of human-derived liver cell lines for the detection of environmental and dietary genotoxicants; current state of knowledge. Toxicology 198, 315-328 (2004).

7 Wilkening, S. \& Bader, A. Influence of culture time on the expression of drug-metabolizing enzymes in primary human hepatocytes and hepatoma cell line HepG2. J Biochem Mol Toxicol 17, 207-213 (2003).

8 Nelson, S. D. Mechanisms of the formation and disposition of reactive metabolites that can cause acute liver injury. Drug Metab Rev 27, 147-177 (1995).

9 Pauli-Magnus, C. \& Meier, P. J. Hepatobiliary transporters and drug-induced cholestasis. Hepatology 44, 778-787 (2006)

10 Fromenty, B. et al. Amiodarone inhibits the mitochondrial beta-oxidation of fatty acids and produces microvesicular steatosis of the liver in mice. J Pharmacol Exp Ther 255, 1371-1376 (1990).

11 Murray, K. F., Hadzic, N., Wirth, S., Bassett, M. \& Kelly, D. Drug-related hepatotoxicity and acute liver failure. J Pediatr Gastroenterol Nutr 47, 395-405 (2008).

12 Rotolo, F. S., Branum, G. D., Bowers, B. A. \& Meyers, W. C. Effect of cyclosporine on bile secretion in rats. Am J Surg 151, 35-40 (1986).

13 Van Summeren, A. et al. Proteomics Investigations of Drug-Induced Hepatotoxicity in HepG2 Cells. Toxicol Sci 120, 109-122 (2011).

14 Van Summeren, A. et al. Screening for drug-induced hepatotoxicity in primary mouse hepatocytes using acetaminophen, amiodarone, and cyclosporin a as model compounds: an omics-guided approach. Omics : a journal of integrative biology 17, 71-83 (2013).

15 Seglen, P. O. Preparation of isolated rat liver cells. Methods Cell Biol 13, 29-83 (1976).

16 Mosmann, T. Rapid colorimetric assay for cellular growth and survival: application to proliferation and cytotoxicity assays. J Immunol Methods 65, 55-63 (1983).

17 Mathijs, K. et al. Discrimination for genotoxic and nongenotoxic carcinogens by gene expression profiling in primary mouse hepatocytes improves with exposure time. Toxicol Sci 112, 374-384 (2009).

18 Jennen, D. G. et al. Comparison of HepG2 and HepaRG by whole-genome gene expression analysis for the purpose of chemical hazard identification. Toxicol Sci 115, 66-79 (2010).

19 Gentleman, R. C. et al. Bioconductor: open software development for computational biology and bioinformatics. Genome biology 5, R80 (2004).

20 Tong, W. et al. ArrayTrack--supporting toxicogenomic research at the U.S. Food and Drug Administration National Center for Toxicological Research. Environmental health perspectives 111, 1819-1826 (2003).

21 Dezso, Z. et al. A comprehensive functional analysis of tissue specificity of human gene expression. BMC Biol 6 , 49 (2008).

22 Boorsma, A., Foat, B. C., Vis, D., Klis, F. \& Bussemaker, H. J. T-profiler: scoring the activity of predefined groups of genes using gene expression data. Nucleic acids research 33, W592-595 (2005).

23 Kienhuis, A. S. et al. Parallelogram approach using rat-human in vitro and rat in vivo toxicogenomics predicts acetaminophen-induced hepatotoxicity in humans. Toxicol Sci 107, 544-552 (2009). 
24 Hockley, S. L., Arlt, V. M., Brewer, D., Giddings, I. \& Phillips, D. H. Time- and concentration-dependent changes in gene expression induced by benzo(a)pyrene in two human cell lines, MCF-7 and HepG2. BMC genomics 7, 260 (2006).

25 Lambert, C. B., Spire, C., Claude, N. \& Guillouzo, A. Dose- and time-dependent effects of phenobarbital on gene expression profiling in human hepatoma HepaRG cells. Toxicology and applied pharmacology 234, 345-360 (2009).

26 Hinson, J. A., Roberts, D. W. \& James, L. P. Mechanisms of acetaminophen-induced liver necrosis. Handb Exp Pharmacol, 369-405 (2010).

27 Belin, M. W., Bouchard, C. S. \& Phillips, T. M. Update on topical cyclosporin A. Background, immunology, and pharmacology. Cornea 9, 184-195 (1990).

28 Van Summeren, A. et al. Screening for Drug-Induced Hepatotoxicity in Primary Mouse Hepatocytes Using Acetaminophen, Amiodarone, and Cyclosporin A as Model Compounds: An Omics-Guided Approach. Omics : a journal of integrative biology (2013).

29 Hewitt, N. J. \& Hewitt, P. Phase I and II enzyme characterization of two sources of HepG2 cell lines. Xenobiotica 34, 243-256 (2004).

30 Zahno, A. et al. The role of CYP3A4 in amiodarone-associated toxicity on HepG2 cells. Biochem Pharmacol 81, 432-441 (2010).

31 Edinger, A. L. \& Thompson, C. B. Death by design: apoptosis, necrosis and autophagy. Curr Opin Cell Biol 16, 663669 (2004).

32 Chiang, J. Y. Bile acids: regulation of synthesis. Journal of lipid research 50, 1955-1966 (2009).

33 Wagner, M., Zollner, G. \& Trauner, M. Nuclear receptor regulation of the adaptive response of bile acid transporters in cholestasis. Seminars in liver disease 30, 160-177 (2010).

34 Wang, Y. et al. Regulation of cholesterologenesis by the oxysterol receptor, LXRalpha. The Journal of biological chemistry 283, 26332-26339 (2008).

35 Andersson, B. S., Rundgren, M., Nelson, S. D. \& Harder, S. N-acetyl-p-benzoquinone imine-induced changes in the energy metabolism in hepatocytes. Chemico-biological interactions 75, 201-211 (1990).

36 Lang, C. et al. Impaired hepatic fatty acid oxidation in rats with short-term cholestasis: characterization and mechanism. Journal of lipid research 42, 22-30 (2001).

37 Ballantyne, C. M. et al. Effects of cyclosporine therapy on plasma lipoprotein levels. Jama 262, 53-56 (1989). 


\section{CHAPTER 6}

\section{In vitro to in vivo comparison of cyclosporin A- induced protein expression profiles}

Anke Van Summeren, Anne Kienhuis, Johan Renes, Freek G. Bouwman, Ewoud N. Speksnijder, Jean-Paul Noben, Joost H. M. van Delft, Jos C. S. Kleinjans and Edwin C. M. Mariman 


\section{ABSTRACT}

In toxicology and pharmacology, hepatotoxic properties of chemicals and drugs are usually analyzed in in vivo repeated-dose toxicity tests, which involve a high number of laboratory animals. To reduce the amount of laboratory animals, the development of alternative in vitro models is necessary. Ideally these in vitro models reflect the in vivo toxicological response and later stage phenotypes such as cholestasis. In this study the protein expression in livers from C57BL/6 mice after cyclosporin A-induced cholestasis was analyzed. The development of cholestasis was examined after 4, 11 and 25 days of a daily cyclosporin A treatment, after 25 days the cholestatic phenotype was established. The data on liver protein expression of this in vivo study enabled an in vitro to in vivo comparison by using the results of our previous studies with HepG2 and primary mouse hepatocytes. The in vivo proteomics data show that cyclosporin A-induced oxidative stress and mitochondrial dysfunction, leading to a decreased mitochondrial ATP production and an altered urea cycle. These processes were also altered by cyclosporin $A$ in the in vitro models HepG2 and primary mouse hepatocytes. In addition detoxification enzymes like methyl- and glutathione-S-transferases were differentially expressed after cyclosporin A treatment. Changes in these detoxification enzymes were mainly detected in vivo, though primary mouse hepatocytes showed a differential expression of some of these enzymes. By means of a functional classification of differentially expressed proteins we demonstrated similarities and differences between in vitro and in vivo models in the proteome response of cyclosporin A-induced hepatotoxicity. The relevance of these results for developing protein signatures in in vitro models predictive for hepatotoxicity in vivo, is discussed. 


\section{INTRODUCTION}

Before their release, novel drugs should be recognized as safe for human exposure. With respect to drug-induced toxicity hepatotoxicity is prominent, because most drugs are metabolized to be eliminated by the liver. The hepatotoxic properties of chemicals and drugs are usually analyzed in in vivo repeated-dose toxicity tests, which involve a high number of laboratory animals. To reduce the amount of laboratory animals, alternative in vitro models are currently developed and their screening properties evaluated ${ }^{1-3}$. Ideally these in vitro models reflect the in vivo toxicological response. Accordingly, in vitro to in vivo comparisons are necessary. By applying 'omics technologies it is possible to measure similar endpoints of druginduced changes between in vitro and in vivo which enables a global comparison of both models ${ }^{4}$. While the conventional assays generally measure only a limited set of biological endpoints, 'omics technologies offers the possibility to measure multiple endpoints simultaneously in a single experiment. Currently, transcriptomics studies, where thousands of genes are measured simultaneously, have shown to be successful for this purpose ${ }^{4-6}$. However, transcriptomics investigates the relative mRNA levels of genes which often only moderately correlate with the relative abundance of their protein product. This moderate correlation is due to turnover differences of proteins and mRNA ${ }^{7}$. In addition, post-translational modifications and protein interactions are not detected by transcriptomics, which emphasizes the need for proteomics. For example, by applying difference gel electrophoresis (DIGE) proteins are separated based on their $\mathrm{pl}$ and molecular weight, so different protein iso-forms can be visualized $^{8}$.

Previously we investigated the proteome of HepG2 cells and primary mouse hepatocytes after exposure to three well-defined hepatotoxicants ${ }^{9,10}$. These were acetaminophen, amiodarone and cyclosporin A, of which cyclosporin A generated the most prominent response. Cyclosporin $A$ is an immunosuppressive drug, however, as an adverse side effect it induces cholestasis caused by the inhibition of the bile salt transporters in hepatocytes ${ }^{11}$.

The aim of the present study is to identify cholestatic-specific mechanisms in vivo, with use of proteomics. Furthermore, we want to compare these results with our previous in vitro studies with HepG2 and primary mouse hepatocytes ${ }^{9,10}$.

To this purpose the hepatic protein expression from $\mathrm{C} 57 \mathrm{BL} / 6$ mice after cyclosporin Ainduced cholestasis was examined. The development of cholestasis at the proteome level was analyzed after 4, 11 and 25 days of a daily doses of cyclosporin A. The cholestatic phenotype was established after 25 days and was confirmed by serum biochemistry and histopathology. DIGE was used to analyze the differentially expressed proteins induced by cyclosporin $A$. The results from our previous studies with HepG $2{ }^{9}$ and primary mouse hepatocytes ${ }^{10}$ were used to establish an in vitro to in vivo comparison of cyclosporin A-induced protein expression profiles. 


\section{MATERIALS AND METHODS}

\section{Chemicals}

Cyclosporin A (CAS-no 59865-13-3, purity minimum $98 \%$ ), was kindly provided by Novartis, Basel, Switzerland. N,N-dimethylformamide (anhydrous, $99.8 \%$ ) was purchased from SigmaAldrich (Zwijndrecht, The Netherlands), the Protein Assay Kit was from Bio-Rad (Veenendaal, The Netherlands). All chemicals used for DIGE were purchased from GE Healthcare (Diegem, Belgium).

\section{Animals}

Male C57BL/6 mice, aged 10 weeks at the start of the treatment period (21-27 g), were obtained from Charles River $\mathrm{GmbH}$, Sulzfeld, Germany. Animals were kept under controlled specific pathogen-free conditions $\left(23^{\circ} \mathrm{C}, 40 \%-50 \%\right.$ humidity) under a $12 \mathrm{~h}$ light-dark cycle, and housed in groups of five. Food and tap water were available ad libitum during the whole experiment. Experiments were conducted at the animal facility of the Leiden University Medical Center, under ethical review in accordance with the Dutch law (approval no. 09157).

\section{Animal treatment}

For the 25 day repeated dose study, forty animals were assigned to eight groups of five mice per group. For the 4 and 11 day repeated-dose study, twenty-four animals were assigned to six groups of four mice per group. After an acclimatization period, treatment protocols were used in which mice were dosed with cyclosporin A in olive oil or with the vehicle only, by oral gavage in a volume of $4 \mathrm{ml} / \mathrm{kg}$ body weight, for five times per week (working days) between 2:00 and 4:00 pm. In the 25 day study, mice were treated with cyclosporin A up to $80 \mathrm{mg} / \mathrm{kg}$ body weight, this study was used for dose range finding, selecting the dose of $26.6 \mathrm{mg} / \mathrm{kg}$ body weight which was determined to be the critical effect dose that induced cholestasis clinical chemistry parameters at 25 days of exposure. The animals were sacrificed by inhalation of $\mathrm{CO}_{2}$ and heart puncture at four, eleven and twenty-five days post cyclosporin $\mathrm{A}$ administration. Blood was collected in $0.8 \mathrm{~mL}$ Minicollect serum collection tubes (Greiner Bio-One, Alphen aan de Rijn, The Netherlands) for serum chemistry analyses. The liver samples were frozen in liquid nitrogen and stored at $-80^{\circ} \mathrm{C}$ until the protein isolation. Liver samples of animals treated with $26.6 \mathrm{mg} / \mathrm{kg}$ body weight in the 25 day dose range finding study and liver samples of the 4 and 11 day study were used for proteome analysis.

\section{Serum biochemistry}

Alanine transaminase (ALT), Aspartate transaminase (AST), Cholesterol (CHOL), total bilirubin (TBIL) and total bile acids (TBA) were analyzed on a Beckman Coulter LX20 Clinical Chemistry Analyzer using Beckman reagent kits (Beckman Coulter B.V., Woerden, The Netherlands) for 
TBIL, and CHOL, and a Dialab reagent kit (DIALAB GmbH, Neudorf, Austria) for TBA. A student's-T test was performed on the results to determine significant differences.

\section{Histopathology}

After $24 \mathrm{~h}$ fixation in $4 \%$ neutral buffered formalin, liver samples were stored in $70 \%$ ethanol until further processing, which included automated dehydration, embedding in paraffin, sectioning at $5 \mu \mathrm{m}$, and staining with hematoxylin and eosin.

\section{Protein extraction}

Liver samples were ground into fine powder in liquid nitrogen and homogenized in DIGE labeling buffer containing $7 \mathrm{M}$ urea, $2 \mathrm{M}$ thiourea, $4 \%$ (w/v) CHAPS and $30 \mathrm{mM}$ Tris- $\mathrm{HCl}$. This mixture was mixed thoroughly and subjected to three cycles of freeze thawing with liquid nitrogen. Unbroken cells or connective tissue were removed from the homogenate by centrifugation at $20000 \mathrm{~g}$ for $60 \mathrm{~min}$ at $10^{\circ} \mathrm{C}$. Supernatant was collected and stored at $-80^{\circ} \mathrm{C}$ until further analysis. Protein concentrations were determined with the Protein Assay Kit from BioRad (Veenendaal, The Netherlands).

\section{DIGE}

The protein labeling and the DIGE were performed as described before ${ }^{9}$. A one-way ANOVA test $(P \leq 0.05)$ was used to select the significant differential spots between the experimental groups. In addition, two way ANOVA-treatment, two-way ANOVA-time, and two-way ANOVAinteraction were computed to assign statistically significant changes in spot intensity due to the treatment alone, time alone and due to both treatment and time. The differentially expressed proteins were excised and identified by matrix assisted laser desorption ionization time of flight tandem mass spectrometry (MALDI-TOF/TOF-MS) according to Bouwman et al ${ }^{12}$. Protein spots that could not be identified by MALDI-TOF/TOF- MS were further analyzed by nano liquid chromatography tandem mass spectrometry (LC-MSMS) on an LCQ Classic (ThermoFinnigan) as described before ${ }^{13}$.

\section{Functional Classification}

The proteins differentially expressed after exposure to cyclosporin A in HepG2 and primary mouse hepatocytes, were retrieved from previous studies ${ }^{9,10}$. The Panther classification system (http://www.pantherdb.org) was used to compare the effect of cyclosporin A upon the protein expression in HepG2, primary mouse hepatocytes and in vivo mouse liver. From each experiment the differentially expressed proteins were uploaded to the Panther classification system. 
Furthermore, the Functional Classification Tool of the DAVID Bioinformatics resource 6.7 (http://david.abcc.ncifcrf.gov/) was used to cluster functionally related proteins. For this purpose the differentially expressed proteins from the three experiments were uploaded and classified with the lowest stringency. Afterwards it was retrieved in which experiment these proteins were differentially expressed.
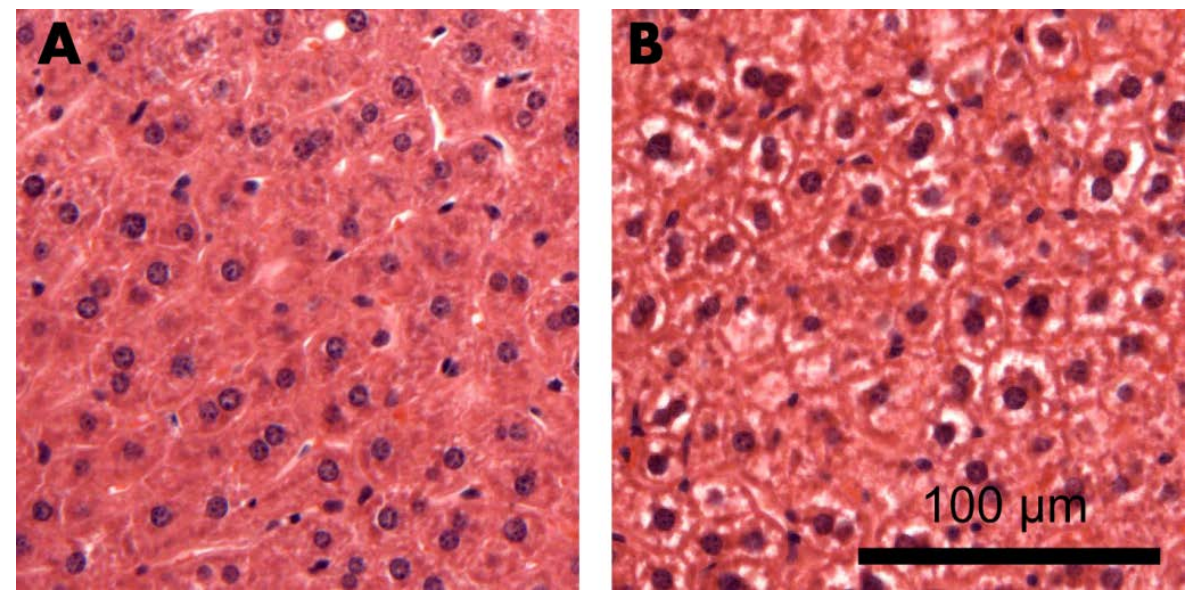

Figure 6.1: Mouse liver treated with vehicle for 25 days is shown in (A). Submembraneous vacuolization suggestive of cholestasis in mouse liver upon treatment with $26.6 \mathrm{mg} / \mathrm{kg}$ body weight for 25 days (B). Magnification: 60X.

\section{RESULTS}

\section{Traditional toxicology parameters}

To induce cholestasis C57BL/6 mice were treated with $26.6 \mathrm{mg} / \mathrm{kg}$ cyclosporin A. Histopathology showed submembraneous vacuolization suggesting cholestasis at 25 days (Figure 6.1).

The plotted serum values per animal for $\mathrm{CHOL}$, TBIL, TBA, ALT and AST are presented in Figure 6.2. Although not always significant dose- and time-dependent increased-responses were observed for the cholestatic parameters $\mathrm{CHOL}$, TBIL, TBA. The general hepatotoxicity markers ALT and ALT did not show an increase at day 4 and 11. However, at day 25 these markers show a trend to an increased expression. 

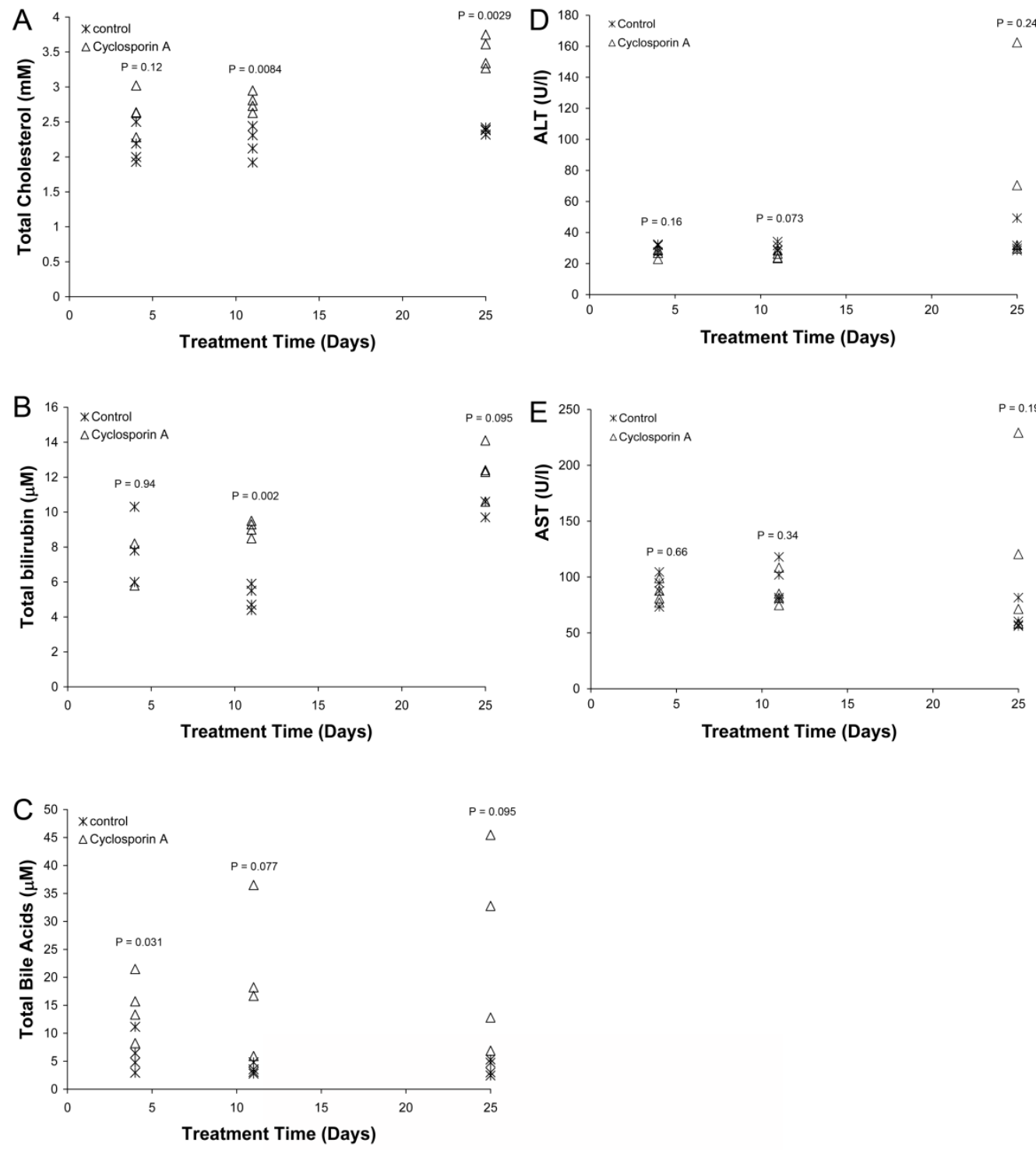

Figure 6.2: Plotted serum values per animal for (A) Cholesterol, (B) Total Bilirubine, (C) Total Bile Acids, (D) ALT and (E) AST at 4,11 , and 25 days of exposure to $26.6 \mathrm{mg} / \mathrm{kg}$ body weight cyclosporin A in Male C57BL/ 6 mice.

\section{DIGE analysis}

To analyze the in vivo hepatotoxic effects of cyclosporin A, C57BL/6 mice were exposed to cyclosporin A for 4,11 and 25 days with olive oil as a vehicle control. On these time-points the animals were sacrificed and the liver was isolated. The proteins were extracted from these 
liver samples and the differentially expressed proteins were determined using DIGE. In total 3235 spots could be matched within all images. With a one-way ANOVA 60 spots were found significantly different $(P \leq 0.05)$ between all groups. With a two-way ANOVA analysis 92 spots were significantly different $(P \leq 0.05)$ in response to treatment, 12 spots were significantly different $(P \leq 0.05)$ in time and 8 spots were differentially expressed for the interaction of the treatment and time $(P \leq 0.05)$.

\section{Protein identification}

The differential spots were included in a pick list. For spot picking and identification a preparative gel was loaded with $150 \mu \mathrm{g}$ of the internal standard labeled with 300 pmol Cy2 and run in the same way as the analytical gels. Afterwards, with use of the DeCyder ${ }^{\mathrm{TM}} 7.0$ software (GE Healthcare) the preparative gel was matched with the analytical gels.

Protein identification was performed by in-gel digestion followed by MALDI-TOF/TOF-MS and/or LC-MS/MS analysis. From the 96 picked spots, all proteins were identified belonging to 81 different proteins. Figure 6.3 shows the 2-DE map made from the master gel with the identified differential spots indicated with a number which corresponds to the numbers presented in Table 6.1. A total of 28 spots were isoforms from 13 proteins due to post-translational modifications or processing of the protein. For the identified protein spots a Tukey's multiple comparison test was performed, from which 4 spots (methylcrotonoyl-CoA carboxylase alpha chain, aconitate hydratase and 2 isoforms of carbamoyl-phosphate synthase) were significantly differential after 4 days treatment of cyclosporin A. After 11 days of treatment 8 spots were differentially expressed (alpha enolase, selenium-binding protein 2, NADP-dependent malic enzyme, transketolase, eukaryotic peptide chain release factor subunit 1 , pyruvate kinase, superoxide dismutase and indolethylamine $\mathrm{N}$-methyltransferase). Twenty five days of treatment induced the differentially expression of 6 spots (glutathione S-transferase Mu 2, farnesyl pyrophosphate synthetase, selenium-binding protein 2, sulfite oxidase, thiopurine Smethyltransferase and protein disulfide-isomerase).

In Table 6.1 the spots are listed with their protein identification and their fold changes between the control and compound. The significant changes (Tukey's multiple comparison test P-value $\leq 0.05$ ) are marked with an asterisk. 


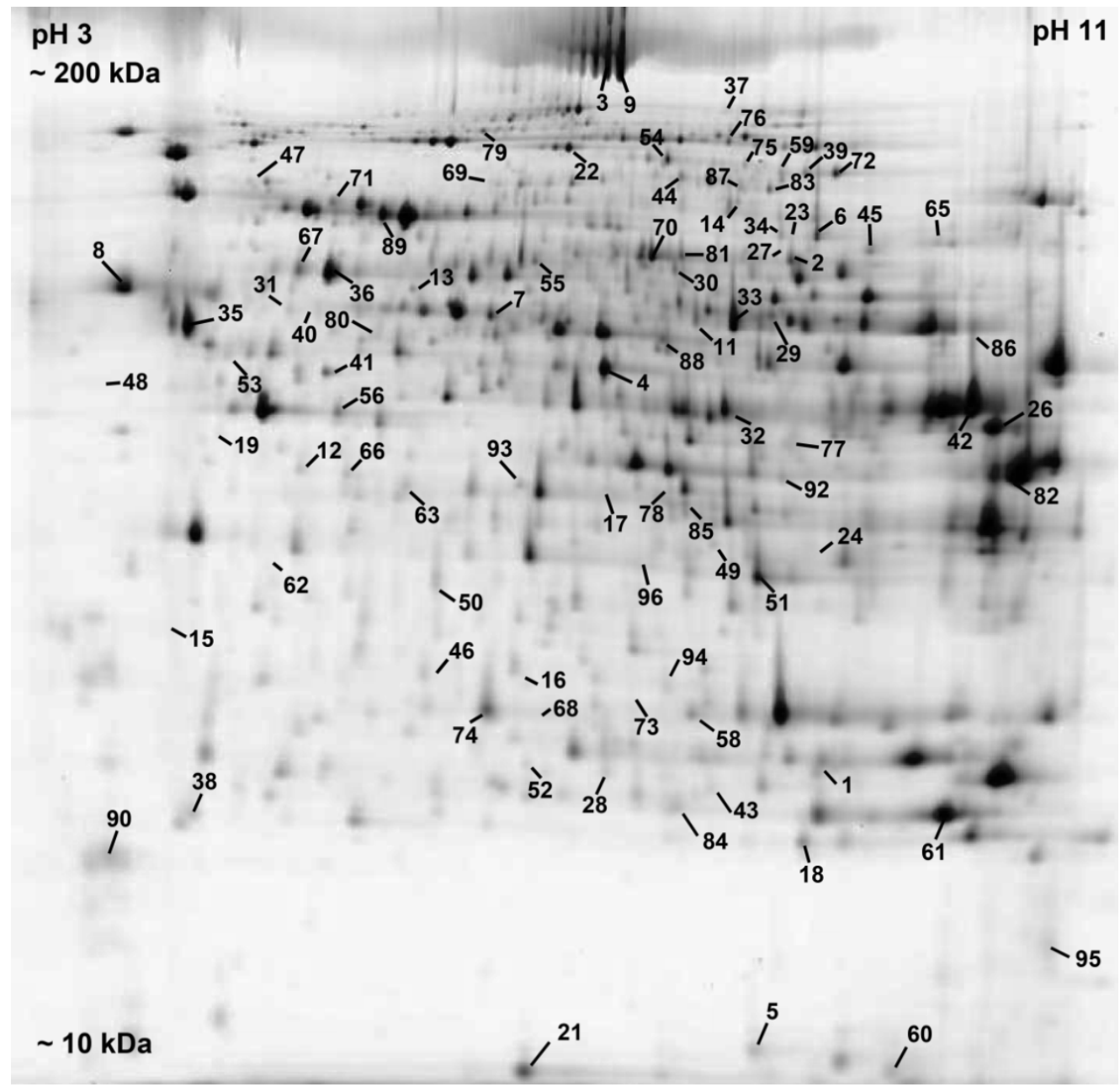

Figure 6.3: Proteome map of the differentially expressed proteins mouse liver after exposure to $26.6 \mathrm{mg} / \mathrm{kg}$ body weight cyclosporin A. The identified spots are indicated with a number which corresponds to the numbers used in Table 6.1.

\section{Functional classification}

Data from HepG2 and primary mouse hepatocytes were retrieved from previous studies ${ }^{9,10}$. The Venn diagram in Figure 6.4 illustrates the overlap of differentially expressed proteins induced by cyclosporin A in in vivo mouse liver, primary mouse hepatocytes and HepG2 cells. In order to compare the protein expression results in mouse and human cells, only mouse orthologues were used. The overlap of the differentially expressed proteins is the highest between the in vitro models PMH 
and HepG2. However the in vivo-in vitro comparison of cyclosporin A-induced hepatotoxicity based on single protein expression shows only a small overlap in the differentially expressed proteins from the different models. For that reason we made use of the Panther classification system to identify the functional properties of the identified proteins. The differentially expressed proteins in the liver from exposed mice were mostly involved in metabolic, immune system processes and generation of precursor metabolites and energy (Figure 6.5). For HepG2 cells and primary mouse hepatocytes the majority of the differential proteins are involved in transport, metabolic and cellular processes (Figure 6.5). Similar processes between the analyzed in vitro systems are cell cycle, cellular processes, developmental processes and cell adhesion (Figure 6.5). In all three models cyclosporin A altered proteins which belong to transport and a response to stimulus (Figure 6.5). The Functional Classification Tool of the DAVID Bioinformatics resource 6.7, revealed 12 clusters which are presented in Table 6.2.

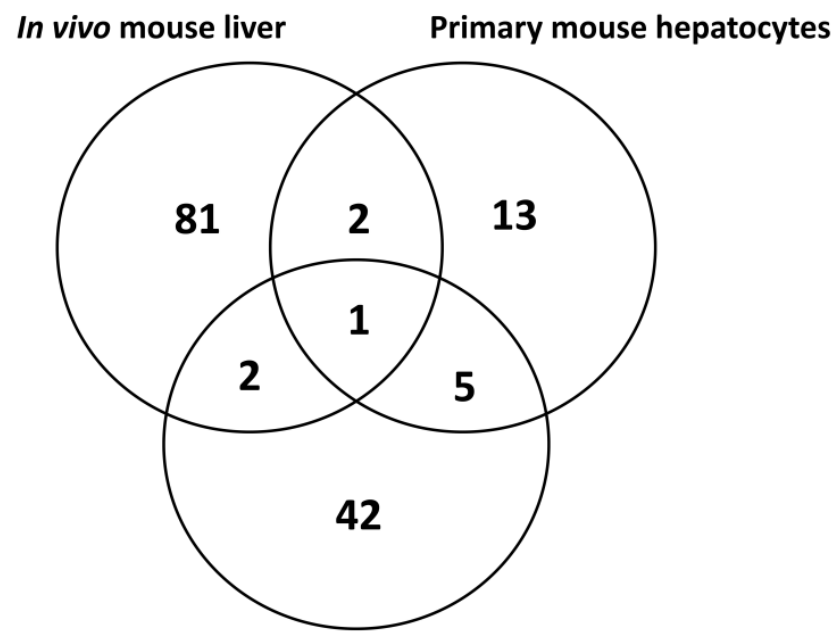

HepG2

Figure 6.4: Venn diagram of the significant differentially expressed proteins induced by cyclosporin A in in vivo mouse liver, HepG2 and primary mouse hepatocytes. 
Table 6.1: Protein identification of differentially expressed proteins in from mouse liver after exposure to CsA after 4, 11 and 25 days.

\begin{tabular}{|c|c|c|c|c|c|c|c|c|}
\hline \multirow[t]{2}{*}{ No. } & \multirow{2}{*}{$\begin{array}{c}\text { Uniprot } \\
\text { Accession } \\
\text { No. }\end{array}$} & \multirow{2}{*}{$\begin{array}{l}\text { Gene } \\
\text { name }\end{array}$} & \multirow[t]{2}{*}{ Protein description } & \multicolumn{4}{|c|}{ P-value } & Fold change $^{5}$ \\
\hline & & & & $\begin{array}{l}\text { one-way } \\
\text { ANOVA }^{1}\end{array}$ & $\begin{array}{l}\text { two-way } \\
\text { ANOVA. } \\
\text { treatment }^{2}\end{array}$ & $\begin{array}{l}\text { two-way } \\
\text { ANOVA. } \\
\text { time }^{3}\end{array}$ & $\begin{array}{l}\text { interaction } \\
\text { treat- } \\
\text { ment/time }\end{array}$ & $\begin{array}{c}\text { CsA4/C4 CsA11/C CsA25/C25 } \\
11\end{array}$ \\
\hline
\end{tabular}

Tricarboxylic acid cycle

\begin{tabular}{|c|c|c|c|c|c|c|c|c|c|c|}
\hline 39 & Q99KI0 & Aco2 & Aconitate hydratase, mitochondrial precursor & 0.018 & 0.0097 & 0.11 & 0.11 & $-1.17^{* *}$ & -1.01 & -1.07 \\
\hline 59 & Q99KI0 & Aco2 & Aconitate hydratase, mitochondrial precursor & 0.13 & 0.02 & 0.93 & 0.23 & -1.13 & -1 & -1.11 \\
\hline 72 & Q99KIO & Aco2 & Aconitate hydratase, mitochondrial precursor & 0.021 & 0.03 & 0.036 & 0.17 & -1.14 & 1 & -1.07 \\
\hline 32 & 088844 & Idh1 & Isocitrate dehydrogenase [NADP] cytoplasmic & 0.038 & 0.0068 & 0.4 & 0.18 & 1.01 & 1.11 & 1.14 \\
\hline 56 & Q97219 & Sucla2 & $\begin{array}{l}\text { Succinyl-CoA ligase [ADP-forming] subunit beta, mito- } \\
\text { chondrial }\end{array}$ & 0.068 & 0.015 & 0.11 & 0.89 & -1.09 & -1.06 & -1.09 \\
\hline 69 & P16332 & Mut & Methylmalonyl-Coenzyme A mutase & 0.01 & 0.028 & 0.2 & 0.29 & -1.04 & -1.14 & -1.33 \\
\hline \multicolumn{11}{|c|}{ Carbohydrate metabolism } \\
\hline 49 & P13707 & Gpd1 & $\begin{array}{l}\text { Glycerol-3-phosphate dehydrogenase }[\mathrm{NAD}+] \text {, cytoplas- } \\
\text { mic }\end{array}$ & 0.062 & 0.014 & 0.093 & 0.9 & 1.1 & 1.09 & 1.13 \\
\hline 82 & Q91Y97 & Aldob & Fructose-bisphosphate aldolase B & 0.087 & 0.042 & 0.6 & 0.083 & -1.05 & 1.16 & 1.13 \\
\hline 4 & P17182 & Eno1 & Alpha enolase & 0.0024 & 0.00028 & 0.47 & 0.052 & 1.02 & $1.11^{* *}$ & 1.05 \\
\hline 25 & Q9QXD6 & Fbp1 & Fructose-1,6-bisphosphatase & 0.001 & 0.0047 & 0.00085 & 0.4 & 1.04 & $1.1^{*}$ & 1.04 \\
\hline 58 & Q9DBJ1. & Pgam1 & Phosphoglycerate mutase 1 & 0.0075 & 0.018 & 0.0047 & 0.54 & 1.07 & 1.02 & 1.06 \\
\hline 30 & P53657 & Pklr & Pyruvate kinase, isozymes $R / L$ & 0.036 & 0.0058 & 0.046 & 0.17 & 1.07 & $1.24^{* *}$ & 1.07 \\
\hline 85 & Q93092 & Taldo1 & Transaldolase & 0.038 & 0.045 & 0.18 & 0.061 & -1.04 & 1.1 & 1.13 \\
\hline 50 & P97328 & Khk & Ketohexokinase & 0.073 & 0.014 & 0.75 & 0.15 & -1.01 & 1.15 & 1.14 \\
\hline 93 & Q9DBB8 & Dhdh & Trans-1,2-dihydrobenzene-1,2-diol dehydrogenase & 0.037 & 0.22 & 0.038 & 0.085 & -1.05 & 1.09 & 1.07 \\
\hline 88 & Q9JLJ2 & Aldh9a1 & 4-trimethylaminobutyraldehyde dehydrogenase & 0.19 & 0.05 & 0.87 & 0.18 & -1.02 & 1.12 & 1.09 \\
\hline \multicolumn{11}{|c|}{ Urea cycle } \\
\hline 17 & Q61176 & Arg1 & Arginase-1 & 0.0069 & 0.0025 & 0.04 & 0.24 & 1.02 & $1.12^{*}$ & 1.11 \\
\hline 78 & Q61176 & Arg1 & Arginase-1 & 0.25 & 0.038 & 0.52 & 0.61 & -1.12 & -1.1 & -1.03 \\
\hline 42 & P16460 & Ass1 & Argininosuccinate synthase & 0.15 & 0.011 & 0.62 & 0.8 & -1.18 & -1.22 & -1.29 \\
\hline 86 & P16460 & Ass1 & Argininosuccinate synthase & 0.3 & 0.049 & 0.65 & 0.55 & -1.12 & -1.16 & -1.35 \\
\hline 3 & Q8C196 & Cps1 & Carbamoyl-phosphate synthase & 0.0049 & 0.00021 & 0.9 & 0.36 & $-1.31^{* *}$ & -1.15 & -1.18 \\
\hline
\end{tabular}




\begin{tabular}{|c|c|c|c|c|c|c|c|c|c|c|}
\hline \multirow[t]{2}{*}{ No. } & \multirow{2}{*}{$\begin{array}{c}\text { Uniprot } \\
\text { Accession } \\
\text { No. }\end{array}$} & \multirow{2}{*}{$\begin{array}{l}\text { Gene } \\
\text { name }\end{array}$} & \multirow[t]{2}{*}{ Protein description } & \multicolumn{4}{|c|}{ P-value } & \multicolumn{3}{|c|}{ Fold change $^{5}$} \\
\hline & & & & $\begin{array}{l}\text { one-way } \\
\text { ANOVA }^{1}\end{array}$ & $\begin{array}{l}\text { two-way } \\
\text { ANOVA. } \\
\text { treatment }^{2}\end{array}$ & $\begin{array}{l}\text { two-way } \\
\text { ANOVA. } \\
\text { time }^{3}\end{array}$ & $\begin{array}{l}\text { interaction } \\
\text { treat- } \\
\text { ment/time }\end{array}$ & $\mathrm{CsA} 4 / \mathrm{C} 4$ & $\begin{array}{l}\text { CsA11/C } \\
11\end{array}$ & CsA25/C25 \\
\hline 9 & Q8C196 & Cps1 & Carbamoyl-phosphate synthase & 0.014 & 0.00081 & 0.52 & 0.37 & $-1.29^{* *}$ & -1.13 & -1.14 \\
\hline 11 & P26443 & Glud1 & Glutamate dehydrogenase 1, mitochondrial precursor & 0.026 & 0.0011 & 0.54 & 0.87 & -1.13 & -1.13 & -1.18 \\
\hline
\end{tabular}

Cholesterol and lipid metabolic processes

\begin{tabular}{|c|c|c|c|c|c|c|c|c|c|c|}
\hline 12 & Q920E5 & Fdps & Farnesyl pyrophosphate synthetase & 0.0032 & 0.0012 & 0.31 & 0.019 & -1.05 & $1.37^{*}$ & $1.65^{* *}$ \\
\hline 66 & Q920E5 & Fdps & Farnesyl pyrophosphate synthetase & 0.064 & 0.026 & 0.091 & 0.51 & 1.07 & 1.13 & 1.32 \\
\hline 19 & P52430 & Pon1 & Serum paraoxonase/arylesterase 1 & 0.048 & 0.0033 & 0.72 & 0.4 & -1.15 & $-1.36^{*}$ & -1.12 \\
\hline 55 & Q9QXE0 & Hacl1 & 2-hydroxyphytanoyl-CoA lyase & 0.097 & 0.015 & 0.38 & 0.41 & 1.05 & 1.15 & 1.07 \\
\hline 45 & P50544 & Acadvl & $\begin{array}{l}\text { Acyl-CoA dehydrogenase, very-long-chain specific, mito- } \\
\text { chondrial precursor }\end{array}$ & 0.02 & 0.012 & 0.019 & 0.87 & -1.08 & -1.13 & -1.11 \\
\hline 81 & Q8VCW8 & Acsf2 & Acyl-CoA synthetase family member 2 , mitochondrial & 0.043 & 0.042 & 0.19 & 0.1 & -1.01 & 1.19 & $1.06^{*}$ \\
\hline 26 & Q8BWT1 & Acaa2 & 3-ketoacyl-CoA thiolase, mitochondrial & 0.041 & 0.0048 & 0.31 & 0.42 & -1.1 & -1.04 & -1.13 \\
\hline 68 & Q8VCC1 & Hpgd & 5-hydroxyprostaglandin dehydrogenase [NAD+] & 0.16 & 0.027 & 0.91 & 0.29 & 1.1 & 1.43 & 1.14 \\
\hline 37 & Q91V92 & Acly & ATP citrate lyase & 0.036 & 0.0088 & 0.38 & 0.083 & -1.01 & $1.22^{*}$ & 1.17 \\
\hline 35 & P56480 & Atp5b & ATP synthase beta chain, mitochondrial precursor & 0.033 & 0.0081 & 0.067 & 0.8 & -1.09 & -1.05 & -1.1 \\
\hline \multicolumn{11}{|c|}{ Protein metabolic processes } \\
\hline 80 & Q8BWY3 & Etf1 & Eukaryotic peptide chain release factor subunit 1 & 0.05 & 0.041 & 0.64 & 0.035 & 1.05 & $-1.42^{* *}$ & -1.1 \\
\hline 43 & P49722 & Psma2 & Proteasome subunit alpha type- 2 & 0.12 & 0.011 & 0.67 & 0.48 & 1.04 & 1.13 & 1.12 \\
\hline 64 & 088685 & Psmc3 & $26 \mathrm{~S}$ protease regulatory subunit $6 \mathrm{~A}$ & 0.12 & 0.023 & 0.88 & 0.18 & 1.01 & 1.13 & $1.36^{*}$ \\
\hline 77 & P62334 & Psmc6 & $26 \mathrm{~S}$ protease regulatory subunit S10B & 0.0032 & 0.038 & 0.000059 & 0.52 & -1.03 & -1.09 & -1.03 \\
\hline 46 & P97371 & Psme1 & Proteasome activator complex subunit 1 & 0.15 & 0.013 & 0.58 & 0.77 & 1.07 & 1.11 & 1.14 \\
\hline 75 & Q9D0R2 & Tars & Threonyl-tRNA synthetase, cytoplasmic & 0.047 & 0.033 & 0.034 & 0.75 & 1.06 & 1.03 & 1.08 \\
\hline \multicolumn{11}{|c|}{ Other metabolic processes } \\
\hline 92 & Q80X81 & Acat3 & acetyl-Coenzyme $A$ acetyltransferase 3 & 0.03 & 0.12 & 0.29 & 0.015 & -1.07 & 1.12 & 1.08 \\
\hline 62 & P97355 & Srm & Spermidine synthase & 0.11 & 0.021 & 0.28 & 0.36 & 1.25 & 1.06 & 1.34 \\
\hline 14 & Q99MR8 & Mccc1 & $\begin{array}{l}\text { Methylcrotonoyl-CoA carboxylase alpha chain, mito- } \\
\text { chondrial precursor }\end{array}$ & 0.0094 & 0.0014 & 0.0049 & 0.08 & $-1.15^{* *}$ & -1.09 & -1.01 \\
\hline 6 & P40142 & Tkt & Transketolase & 0.00088 & 0.00044 & 0.0091 & 0.16 & 1.03 & $1.14^{* *}$ & 1.13 \\
\hline 23 & P40142 & Tkt & Transketolase & 0.014 & 0.004 & 0.11 & 0.29 & 1.14 & $1.5^{*}$ & 1.25 \\
\hline
\end{tabular}




\begin{tabular}{|c|c|c|c|c|c|c|c|c|c|c|}
\hline \multirow[t]{2}{*}{ No. } & \multirow{2}{*}{$\begin{array}{c}\text { Uniprot } \\
\text { Accession } \\
\text { No. }\end{array}$} & \multirow{2}{*}{$\begin{array}{l}\text { Gene } \\
\text { name }\end{array}$} & \multirow[t]{2}{*}{ Protein description } & \multicolumn{4}{|c|}{ P-value } & \multicolumn{3}{|c|}{ Fold change $^{5}$} \\
\hline & & & & $\begin{array}{l}\text { one-way } \\
\text { ANOVA }^{1}\end{array}$ & $\begin{array}{l}\text { two-way } \\
\text { ANOVA. } \\
\text { treatment }^{2}\end{array}$ & $\begin{array}{l}\text { two-way } \\
\text { ANOVA. } \\
\text { time }^{3}\end{array}$ & $\begin{array}{l}\text { interaction } \\
\text { treat- } \\
\text { ment/time }\end{array}$ & CsA4/C4 & $\begin{array}{l}\text { CsA11/C } \\
11\end{array}$ & CsA25/C25 \\
\hline 22 & Q99LB7 & Sardh & Sarcosine dehydrogenase, mitochondrial precursor & 0.026 & 0.0038 & 0.11 & 0.72 & -1.07 & -1.05 & -1.1 \\
\hline 54 & Q9DBT9 & $\begin{array}{l}\text { ME2GLYD } \\
\mathrm{H}\end{array}$ & $\begin{array}{l}\text { Dimethylglycine dehydrogenase, mitochondrial precur- } \\
\text { sor }\end{array}$ & 0.17 & 0.015 & 0.58 & 0.73 & -1.14 & -1.06 & -1.09 \\
\hline 27 & Q8VC30 & Dak & $\begin{array}{l}\text { Bifunctional ATP-dependent dihydroxyacetone ki- } \\
\text { nase/FAD-AMP lyase }\end{array}$ & 0.023 & 0.0048 & 0.42 & 0.1 & 1 & $1.28^{*}$ & 1.26 \\
\hline 70 & Q8VC30 & Dak & $\begin{array}{l}\text { Bifunctional ATP-dependent dihydroxyacetone ki- } \\
\text { nase/FAD-AMP lyase }\end{array}$ & 0.077 & 0.028 & 0.22 & 0.24 & 1 & 1.14 & 1.08 \\
\hline 63 & Q9CWS0 & Ddah1 & NG,NG-dimethylarginine dimethylaminohydrolase 1 & 0.12 & 0.021 & 0.14 & 0.79 & 1.05 & 1.1 & 1.07 \\
\hline 24 & P52196 & Tst & Thiosulfate sulfurtransferase & 0.0097 & 0.0041 & 0.025 & 0.5 & 1.19 & 1.38 & 1.07 \\
\hline 94 & P00920 & $\mathrm{Ca} 2$ & Carbonic anhydrase 2 & 0.000071 & 0.32 & 0.000005 & 0.98 & 1.06 & 1.07 & 1.04 \\
\hline 10 & Q78JT3 & Haao & 3-hydroxyanthranilate 3,4-dioxygenase & 0.012 & 0.001 & 0.46 & 0.22 & 1.03 & 1.13 & $1.14^{*}$ \\
\hline 76 & Q922D8 & Mthfd1 & C-1-tetrahydrofolate synthase, cytoplasmic & 0.076 & 0.034 & 0.2 & 0.21 & -1.02 & 1.21 & 1.29 \\
\hline \multicolumn{11}{|c|}{ Chaperone } \\
\hline 71 & P38647 & GRP 75 & Stress-70 protein, mitochondrial precursor & 0.0013 & 0.03 & 0.00034 & 0.58 & -1.11 & -1.03 & -1.11 \\
\hline 36 & P63038 & Hspd1 & $60 \mathrm{kDa}$ heat shock protein, mitochondrial precursor & 0.013 & 0.0085 & 0.014 & 0.78 & -1.09 & -1.11 & -1.06 \\
\hline 67 & P63038 & Hspd1 & $60 \mathrm{kDa}$ heat shock protein, mitochondrial precursor & 0.23 & 0.027 & 0.44 & 0.89 & -1.16 & -1.12 & -1.09 \\
\hline 57 & Q8CGK3 & Lonp1 & Lon protease homolog & 0.16 & 0.017 & 0.87 & 0.41 & -1.08 & -1.04 & -1.16 \\
\hline 79 & Q8CGK3 & Lonp1 & Lon protease homolog & 0.064 & 0.038 & 0.083 & 0.38 & -1.01 & -1.04 & -1.08 \\
\hline 60 & P17742 & Ppia & Peptidyl-prolyl cis-trans isomerase & 0.08 & 0.021 & 0.23 & 0.33 & 1.04 & 1.19 & 1.07 \\
\hline 95 & P24369 & Ppib & Peptidyl-prolyl cis-trans isomerase B & 0.021 & 0.36 & 0.079 & 0.014 & -1.03 & -1.23 & 1.14 \\
\hline 8 & P09103 & P4hb & Protein disulfide-isomerase & 0.005 & 0.00081 & 0.059 & 0.38 & 1.17 & 1.1 & $1.27^{* *}$ \\
\hline \multicolumn{11}{|c|}{ Secreted } \\
\hline 89 & P07724 & Alb & Serum albumin precursor & 0.00019 & 0.061 & 0.000047 & 0.12 & -1.1 & 1.03 & -1.18 \\
\hline 44 & Q921I1 & Tf & Serotransferrin precursor & 0.01 & 0.011 & 0.012 & 0.45 & -1.06 & -1.26 & -1.19 \\
\hline 83 & Q921I1 & Tf & Serotransferrin precursor & 0.036 & 0.043 & 0.35 & 0.033 & 1.13 & -1.29 & $-1.4^{*}$ \\
\hline 87 & Q921I1 & Tf & Serotransferrin precursor & 0.15 & 0.049 & 0.94 & 0.12 & 1.05 & -1.21 & -1.31 \\
\hline \multirow[t]{2}{*}{90} & P04938 & \multicolumn{9}{|c|}{ Mup8 and Major urinary proteins 11 and 8} \\
\hline & & 10 & & 0.043 & 0.076 & 0.082 & 0.19 & -1.07 & -1.7 & -1.05 \\
\hline
\end{tabular}




\begin{tabular}{|c|c|c|c|c|c|c|c|c|}
\hline \multirow[t]{2}{*}{ No. } & Uniprot & Gene & Protein description & & & -value & & Fold change $^{5}$ \\
\hline & $\begin{array}{l}\text { Accession } \\
\text { No. }\end{array}$ & name & & $\begin{array}{l}\text { one-way } \\
\text { ANOVA }^{1}\end{array}$ & $\begin{array}{l}\text { two-way } \\
\text { ANOVA. } \\
\text { treatment }^{2}\end{array}$ & $\begin{array}{l}\text { two-way } \\
\text { ANOVA. } \\
\text { time }^{3}\end{array}$ & $\begin{array}{l}\text { interaction } \\
\text { treat- } \\
\text { ment/time }\end{array}$ & $\begin{array}{c}\text { CsA4/C4 CsA11/C CsA25/C25 } \\
11\end{array}$ \\
\hline
\end{tabular}

cytoskeleton

\section{$29 \quad \mathrm{P} 40124$}

$20 \quad \mathrm{P} 68134$

Cap1

Acta1

Adenylyl cyclase-associated protein 1

Actin, alpha skeletal muscle

Xenobiotic metabolism

$84 \quad \mathrm{P} 24472$

$1 \quad \mathrm{P} 15626 \quad$ Gstm2

$52035660 \quad$ Gstm6

61 P19157 Gstp1

28 Q9WVLO Gstz1

21 P08228 Sod1

Q9QXF8

96 Q9QXF8 Gnmt

$74 \quad \mathrm{P} 40936 \quad$ Inmt

$16055060 \quad$ Tpmt

15 Q91VF2 Hnmt

$34 \quad$ Q60967 Papss1

33 P26443 Glud1

Glutathione S-transferase 5.7

Glutathione S-transferase Mu 2

Glutathione S-transferase Mu 6

Glutathione S-transferase P 1

Maleylacetoacetate isomerase

Superoxide dismutase [Cu-Zn]

Glycine N-methyltransferase

Glycine N-methyltransferase

Indolethylamine N-methyltransferase

Thiopurine S-methyltransferase

Histamine N-methyltransferase

Adenosylhomocysteinase

Bifunctional 3'-phosphoadenosine 5'-phosphosulfate

synthase 2

Glutamate dehydrogenase 1, mitochondrial precursor
51 Q9QXF8 Gnmt

$91 \quad \mathrm{P} 50247$ Ahcy

Superoxide dismutase

\subsection{2}

0.048

0.0054

0.0033

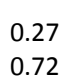

0.13

0.044

$0.00004 \quad 5.1 \mathrm{E}-06$

$0.11 \quad 0.015$

$0.078 \quad 0.021$

$0.032 \quad 0.0052$

$0.042 \quad 0.0034$

$0.033 \quad 0.0031$

$0.047 \quad 0.014$

$0.023 \quad 0.9$

$0.094 \quad 0.031$

$0.02 \quad 0.0018$

0.038

0.0044

0.038

0.0043

0.001

0.092

0.0075

0.0068

Programmed cell death protein 8 , mitochondrial precur-

Apoptosis

$\begin{array}{lll}65 & \text { Q970X1 } & \text { Aifm1 } \\ 47 & \text { Q91VD9 } & \text { Ndufs1 } \\ 53 & \text { P05784 } & \text { Krt18 }\end{array}$

$\mathrm{NADH}$-ubiquinone oxidoreductase $75 \mathrm{kDa}$ subunit,

Not listed

$\begin{array}{lllllll}0.074 & 0.024 & 0.012 & 0.51 & -1.11 & -1.03 & -1.05 \\ 0.0063 & 0.013 & 0.012 & 0.13 & -1.09 & -1.01 & -1.17^{*} \\ 0.063 & 0.015 & 0.23 & 0.31 & 1.03 & 1.18 & 1.27\end{array}$




\begin{tabular}{|c|c|c|c|c|c|c|c|c|c|c|}
\hline \multirow[t]{2}{*}{ No. } & \multirow{2}{*}{$\begin{array}{c}\text { Uniprot } \\
\text { Accession } \\
\text { No. }\end{array}$} & \multirow{2}{*}{$\begin{array}{l}\text { Gene } \\
\text { name }\end{array}$} & \multirow[t]{2}{*}{ Protein description } & \multicolumn{4}{|c|}{ P-value } & \multicolumn{3}{|c|}{ Fold change $^{5}$} \\
\hline & & & & $\begin{array}{l}\text { one-way } \\
\text { ANOVA }^{1}\end{array}$ & $\begin{array}{l}\text { two-way } \\
\text { ANOVA. } \\
\text { treatment }^{2}\end{array}$ & $\begin{array}{l}\text { two-way } \\
\text { ANOVA. } \\
\text { time }^{3}\end{array}$ & $\begin{array}{l}\text { interaction } \\
\text { treat- } \\
\text { ment/time }\end{array}$ & $\mathrm{CsA} 4 / \mathrm{C} 4$ & $\begin{array}{l}\text { CsA11/C } \\
11\end{array}$ & CsA25/C25 \\
\hline 2 & P06801 & Me1 & NADP-dependent malic enzyme & 0.0029 & 0.00019 & 0.13 & 0.53 & 1.16 & $1.26^{* *}$ & 1.14 \\
\hline 40 & Q00896 & Serpina1c & Alpha-1-antitrypsin 1-3 & 0.11 & 0.0098 & 0.62 & 0.56 & -1.1 & -1.34 & -1.29 \\
\hline 31 & Q00897 & Serpina1d & Alpha-1-antitrypsin 1-4 precursor & 0.004 & 0.0065 & 0.0033 & 0.79 & -1.13 & -1.2 & -1.14 \\
\hline 73 & P00920 & $\mathrm{Ca} 2$ & Carbonic anhydrase 2 & 0.002 & 0.031 & 0.000033 & 0.62 & 1.1 & 1.21 & 1.07 \\
\hline 5 & Q01768 & Nme2 & Nucleoside diphosphate kinase B & 0.0053 & 0.00028 & 0.2 & 0.69 & 1.11 & 1.08 & $1.13^{*}$ \\
\hline 7 & Q63836 & Selenbp2 & Selenium-binding protein 2 & 0.001 & 0.00079 & 0.29 & 0.0043 & -1.03 & $1.16^{* *}$ & $1.16^{* *}$ \\
\hline 48 & Q9QYG0 & Ndrg2 & Isoform 1 of Protein NDRG2 & 0.14 & 0.013 & 0.8 & 0.42 & -1.09 & $1.06^{*}$ & -1.3 \\
\hline 13 & Q8R086 & Suox & Sulfite oxidase, mitochondrial precursor & 0.00061 & 0.0013 & 0.0013 & 0.22 & -1.09 & -1.08 & $-1.22^{* *}$ \\
\hline 41 & Q9CZ13 & Uqcrc1 & $\begin{array}{l}\text { Ubiquinol-cytochrome-c reductase complex core } \\
\text { protein I, mitochondrial precursor }\end{array}$ & 0.15 & 0.01 & 1 & 0.57 & 1.08 & -1.03 & -1.06 \\
\hline 38 & P70296 & Pebp1 & Phosphatidylethanolamine-binding protein 1 & 0.079 & 0.0093 & 0.22 & 0.97 & 1.13 & 1.15 & 1.14 \\
\hline
\end{tabular}

${ }^{1} \mathrm{P}$-value from one way ANOVA statistical test between the six groups with each four biological replicates.

${ }^{2}$ P-value from two way ANOVA (treatment) statistical test between the six groups, which indicates the differences between the control and exposed groups.

${ }^{3}$ P-value from two way ANOVA (time) statistical test between the six groups, which indicates the differences between the day 4,11 and 25.

${ }^{4} \mathrm{P}$-value from two way ANOVA (interaction) statistical test between the six groups, which indicates the interaction between time and treatment.

${ }^{5}$ The difference in the standardized abundance of the proteins is expressed as the fold change between the control (C) and the treated groups (T). The fold change is calculated by taking the means of standardized volume values for the protein spot in the corresponding groups $(C=\operatorname{control}, \mathrm{Cs} A=\mathrm{cyclosporin} A, 4=$ day $4,11=$ day $11,25=$ day 25$)$, values are calculated as T/C and displayed in the range of +1 to $+\infty$ for increases in expression and calculated as $-\mathrm{C} / \mathrm{T}$ and displayed in the range of $-\infty$ to -1 for decreased expression.

${ }^{* *}$ Indicates significant fold changes $(\mathrm{P} \leq 0.05)$ between the control and the treated group, calculated with a multiple comparison test.

* Indicates significant fold changes $(\mathrm{P} \leq 0.1)$ between the control and the treated group, calculated with a multiple comparison test. 


\section{DISCUSSION}

The aim of this study was to identify cholestatic-specific mechanisms in vivo, with use of proteomics. In addition, an in vitro to in vivo comparison of cyclosporin A-induced protein expression profiles was established by comparing these results with the results from our previous in vitro studies with HepG2 and primary mouse hepatocytes ${ }^{9,10}$. To this purpose, we analyzed the hepatic protein expression in C57BL/6 mice after cyclosporin A-induced cholestasis. The cholestatic phenotype was established after 25 days and confirmed by histopathology and serum parameters, which allowed us to search for cholestatic-specific mechanisms in vivo.

\section{Differential protein expression in mice after cyclosporin A treatment}

Cyclosporin $A$ inhibits the bile salt export pump (ABCB11), multidrug resistance protein 2 $(A B C C 2)$ and P-glycoprotein $(A B C B 1)$ in the canalicular membrane vesicles. These ATP Binding Cassette transporters ( $A B C$ transporters) are responsible for the bile secretion into the bile canaliculus ${ }^{14}$. Therefore, inhibition of these transport proteins cause the hepatic accumulation of bile salts resulting in cholestasis ${ }^{15}$. Previous studies suggest that accumulated bile acids induce oxidative stress and can cause mitochondrial dysfunction in the liver ${ }^{16}$. Moreover, cyclosporin A induced in vitro as well as in vivo oxidative stress, increases lipid peroxidation and depletes the hepatic pool of glutathione ${ }^{17,18}$. Superoxide is one of the main reactive oxygen species in the cell which can be converted into oxygen and hydrogen peroxide by superoxide dismutase. In our study cytoplasmatic superoxide dismutase 1 was up-regulated after cyclopsorin A treatment, while mitochondrial superoxide dismutase 2 was downregulated, indicating that cyclosporin $A$ induced oxidative stress and mitochondrial dysfunction. Indications for mitochondrial dysfunction are already visible after 4 days of cyclosporin A treatment, since all proteins with a significantly differential expression are mitochondrial.

Furthermore, the down-regulation of several enzymes of the TCA-cycle like aconitate hydratase, succinyl-CoA ligase [ADP-forming] subunit beta and methylmalonyl-coenzyme A mutase suggest a deficient ATP production by the mitochondria. Previously, others have demonstrated an ATP reduction in hepatocytes after exposure to necrotic concentrations of toxic bile salts ${ }^{19,20}$. The reduced ATP was directly due to mitochondrial dysfunction as glycolytic ATP generation was intact ${ }^{20}$. In our study several proteins from the glycolysis were found to be up-regulated. This suggests a compensative mechanism for mitochondrial dysfunction via the glycolytic pathway.

Glutamate dehydrogenase is responsible for the conversion of glutamate to $\alpha$-ketoglutarate and ammonium, which will bled off to the urea-cycle. The first step of the urea cycle requires ATP for the conversion of $\mathrm{NH}_{4}^{+}$and $\mathrm{HCO}_{3}{ }^{-}$to carbamoyl phosphate, catalyzed by carbamoylphosphate synthase (Cps1). In a later step, ATP is necessary for the conversion of citrulline and aspartate in argininosuccinate, catalyzed by argininosuccinate synthase (Ass1). In our 
study glutamate dehydrogenase 1, Ass1 and Cps1, were all down-regulated together with other enzymes from the urea cycle.

Rare autosomal recessive disorders of the urea cycle like arginase deficiency and citrin deficiency are associated with neonatal intrahepatic cholestasis ${ }^{21,22}$. Neonatal intrahepatic cholestasis is defined as impaired bilirubin excretion, resulting in jaundice and conjugated hyperbilirubinemia, detected either in a newborn or an infant up to 4 months old ${ }^{21,23}$. The pathogenesis of cholestasis in these urea cycle disorders remains unclear, probably the combination of a primary mitochondrial defect and a delayed maturity of bile acid metabolism, may form a vicious circle in transient neonatal intrahepatic cholestasis ${ }^{22}$. A similar mechanism for cholestasis as observed in neonatal intrahepatic cholestasis may explain down-regulation of enzymes from the urea cycle in our study.

Methylation enzymes indolethylamine $N$-methyltransferase (Inmt), thiopurine $S$ methyltransferase, glycine $N$-methyltransferase and histamine $N$-methyltransferase showed an increased expression after cyclosporin A treatment. Methyl conjugation is mainly used for the metabolism of small endogenous compounds such as epinephrine, norepinephrine, dopamine, and histamine but is also involved in the metabolism of macromolecules such as nucleic acids and in the biotransformation of certain drugs ${ }^{24}$. In contrast to other conjugative reactions, methylation leads to less polar compounds that may be less readily excreted from the body ${ }^{24}$. Inmt, thiopurine $S$-methyltransferase, glycine $N$-methyltransferase and histamine $\mathrm{N}$-methyltransferase all use $S$-adenosylmethionine as methyldonor. Previously, it was shown that $S$-adenosylmethionine protects against cyclosporin $A{ }^{25,26}$, chlorpromazine- ${ }^{27}$ and ethenylestradiol-induced cholestasis ${ }^{28}$. Cholestatic rat liver, induced by common bile duct ligation, exhibited increased enzymatic activities of Inmt and thiol methyltransferase ${ }^{29}$.

The increased expression of these $N$-methyltransferases results in an increased conversion of $S$-adenosylmethionine to $S$-adenosyl-homocysteine, which in turn is converted in homocysteine and adenosine by adenosylhomocysteinase, here differentially expressed. The increased conversion of $S$-adenosylmethionine and $S$-adenosyl-homocystein activates the transsulfuration pathway, leading to the formation of glutathione ${ }^{27}$. Glutathione is responsible for the detoxification of various compounds to protect the cells from oxidative stress. Moreover it plays an important role in bile formation ${ }^{30}$. Previously it has been demonstrated that glutathione is depleted during cholestasis, therefore activation of the trans-sulfuration pathway is necessary to maintain the glutathione levels in the cholestasic liver ${ }^{30,31}$. In addition, sulfite oxidase and Bifunctional 3'-phosphoadenosine 5'-phosphosulfate synthase 2 other enzymes of the trans-sulfuration pathway were also differentially expressed.

Glutathione is a substrate of both conjugation and reduction reactions, catalyzed by glutathione $S$-transferase enzymes. Mice exposed to cyclosporin A, show a differential expression of several glutathione $S$-transferases (GSTs). GSTs are not only important phase II detoxification enzymes, they are able to bind bile acids and are thought to play a role in the intracellular trafficking of bile acids ${ }^{32}$. 
Furthermore, cyclosporin A induced the differential expression of proteins related to cholesterol biosynthesis and lipid metabolism. Bile acid synthesis is the main route for cholesterol metabolism and is initiated by cholesterol 7 $\alpha$-hydroxylase (CYP7A1). In our study Farnesyl diphosphate synthase (Fdps) showed an increased expression after 11 days of cyclosporin A exposure. Fdps is responsible for the formation of farnesyldiphosphate, a key intermediate in cholesterol synthesis and protein farnesylation. Previously drug-induced cholestasis was associated with an increased hepatic cholesterol synthesis, which is in line with the presently observed Fdps expression ${ }^{33}$.

In addition, cholesterol synthesis requires the presence of cytosolic acetyl-coA. Previously, we mentioned a down-regulation of the TCA-cycle, a mitochondrial source of acetyl-coA. The upregulation of citrate-lyase and NADP-dependent malic enzyme in our study, suggests a drain of mitochondrial acetyl-coA to the cytosol for cholesterol synthesis.

Furthermore, an increased cholesterol synthesis is associated with a decreased CYP7A1 expression as a protective adaptive response to reduce cellular bile accumulation ${ }^{33}$ Probably these two mechanisms are the cause of high plasma cholesterol in cholestasis. In a previous study, in which transcriptomics analysis was performed on liver samples used for proteome analysis in this study, CYP7A1 down-regulation was observed as one of the strongest effects upon progression of cholestasis in mice and was interpreted as an adaptive response ${ }^{34}$.

Serum paraoxonase/arylesterase 1 (Pon1) is an antioxidant enzyme responsible for the detoxification of organophosphates and prevention of low-density lipoprotein oxidative modification. Our study shows a down-regulation of Pon1 after cyclosporin A treatment. Rats treated with $\mathrm{CCl}_{4}$ showed a reduced activity of hepatic Pon1 together with increased lipid peroxidation ${ }^{35}$ Furthermore, Pon 1 was down-regulated in the protein extract from rat liver exposed to acetaminophen ${ }^{36}$ However, Pon1 failed as candidate marker for drug-induced hepatotoxicity, because it was not consistently altered in response to several hepatotoxicants ${ }^{37}$.

\section{In vivo to in vitro comparison}

Previously we analyzed cyclosporin A-induced cholestasis in HepG2 cell ${ }^{9}$ and in primary mouse hepatocytes ${ }^{10}$. An in vivo to in vitro comparison of cyclosporin A-induced hepatotoxicity based on single protein expression is difficult, partly because there is only a small overlap in the differentially expressed proteins from the different models (Figure 6.4). Therefore the differentially expressed proteins from these models were classified based on the GO-terms with the Panther classification system. 


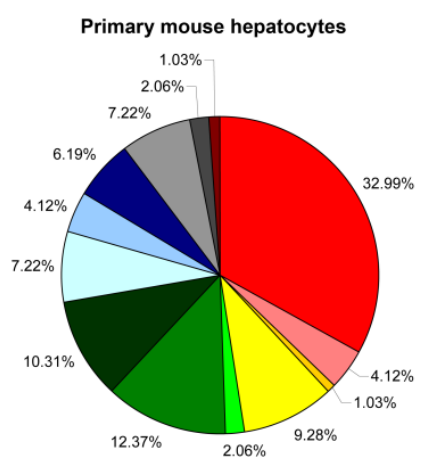

ametabolic process

$\square$ cell adhesion

$\square$ cell communication

a transport

$\square$ system process

- response to stimulus

घeneration of precursor metabolites and energy

aapoptosis
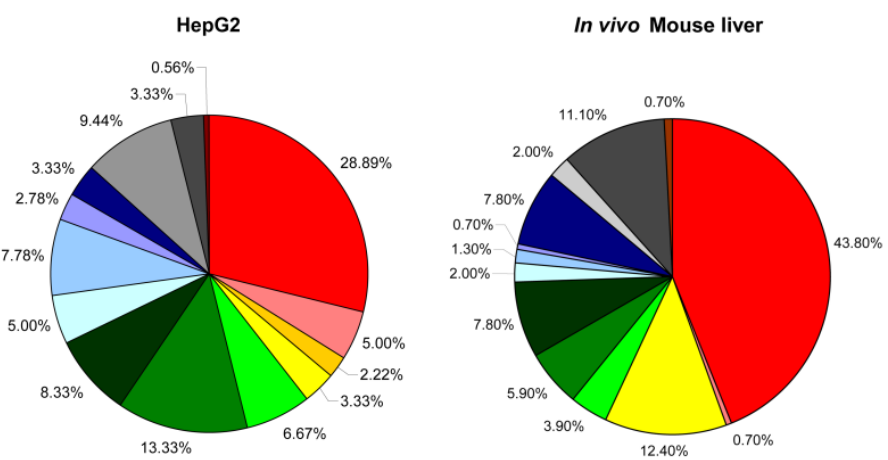

$\square$ cell cycle

$\square$ immune system process

$\square$ cellular process

$\square$ cellular component organization

$\square$ reproduction

$\square$ developmental process

घunkown

Figure 6.5: Classification of the differential expressed proteins in primary mouse hepatocytes, HepG2 and in vivo liver after exposure to cyclosporin A with the Panther classification system (http://www.pantherdb.org).

This classification revealed a similar outcome for HepG2 and primary mouse hepatocytes. The majority of the differentially expressed proteins are involved in metabolic processes, cellular processes and transport. Similar processes between the analyzed in vitro systems are cell cycle, cellular processes, developmental processes and cell adhesion (Figure 6.5). In all three models cyclosporin A altered proteins belonging to transport and a response to stimulus (Figure 6.5). For a more detailed overview the proteins were clustered according to their function with DAVID Bioinformatics Resources 6.7. Cluster 1 and 2 contain differentially expressed proteins from the three models. Cluster 1 involves ATP-binding and mitochondrial proteins and cluster 2 contains proteins from carbohydrate metabolism. These clusters show that cyclosporin A induces in vivo as well as in vitro mitochondrial dysfunction and changes in the energy metabolism, as also described before.

Mitochondria are critical targets for drug toxicity, therefore mitochondrial dysfunction and oxidative stress are often seen in drug-induced hepatotoxicity ${ }^{38}$. The differential expression of proteins from energy metabolism are probably a first indication of a toxicological response.

However, several proteins from energy metabolism are often detected in comparative proteomics and considered as proteins from a general stress response ${ }^{39}$. Therefore using those proteins as specific markers for cholestasis should be done with caution. Methyltransferases which are described earlier as detoxifying enzymes constitute cluster 4. Almost all methyltransferases were detected only in vivo, however Inmt was also detected in primary mouse hepatocytes. Furthermore, in vivo cyclosporin A induced cholestasis was accompanied with the differential expression of glutathione-S-transferases (cluster 7). In primary mouse hepatocytes we have also detected some glutathione-S-transferases, although they were not signifi- 
cantly changed after cyclosporin A treatment and were therefore excluded from the cluster analysis. We believe that both methyltransferases and glutathione-S-transfereses are important detoxification proteins in cyclosporin A-induced cholestasis. These proteins were mainly detected in vivo but some also in primary mouse hepatocytes. However, they were not observed in HepG2, indicating the minority of drug-metabolizing enzymes in HepG2 ${ }^{40,41}$.

Table 6.2: Gene classification of the differentially expressed proteins after cyclosporin A treatment in vivo, primary mouse hepatocytes and HepG2, performed with the Functional Classification Tool of the DAVID Bioinformatics resource 6.7 (http://david.abcc.ncifcrf.gov/).

\begin{tabular}{|c|c|c|c|c|c|}
\hline Cluster & Function & $\begin{array}{l}\text { Enrichment } \\
\text { Score }\end{array}$ & in vivo & $\mathrm{PMH}$ & HepG2 \\
\hline 1 & ATP-binding & 9.62041 & 40 & 5 & 16 \\
\hline 2 & Carbohydrate metabolism & 9.293973 & 8 & 1 & 9 \\
\hline 3 & metal binding & 7.999105 & 10 & 1 & 1 \\
\hline 4 & Methyltransferases & 5.247008 & 4 & 1 & 0 \\
\hline 5 & metal binding & 4.844126 & 8 & 2 & 5 \\
\hline 6 & NAD cofactor & 4.807059 & 1 & 0 & 3 \\
\hline 7 & $\begin{array}{l}\text { Detoxification glutathione } \\
\text { S-transferase }\end{array}$ & 4.368559 & 5 & 0 & 0 \\
\hline 8 & Chaperone activity & 3.910156 & 1 & 7 & 9 \\
\hline 9 & Cytoskelet & 3.231529 & 1 & 1 & 5 \\
\hline 10 & mRNA processing & 2.748475 & 0 & 0 & 5 \\
\hline 11 & Protein transport & 1.503542 & 1 & 1 & 1 \\
\hline 12 & $\begin{array}{l}\text { response to organic sub- } \\
\text { strate }\end{array}$ & 0.489311 & 2 & 1 & 0 \\
\hline
\end{tabular}

Characteristic for HepG2 cells, proteins responsible for mRNA processing were differentially expressed (cluster 10). These proteins have central roles in DNA repair, telomere elongation, cell signaling and in regulating gene expression at transcriptional and translational level ${ }^{42}$. Furthermore, heterogeneous nuclear ribonucleoproteins (hnRNPs) play a role in tumor development and are up-regulated in various cancers ${ }^{42}$. Previously it was shown that carcinoma cell lines including HepG2 cells have a higher expression of hnRNPs than human intestinal epithelium ${ }^{43}$. Therefore the expression of these proteins can be ascribed to the carcinoma character of the HepG2 cell line. Apparently cyclosporin A induced a down-regulation of these proteins and probably decreased cell proliferation.

In vitro cyclosporin A mainly induced the differentially expression of chaperone proteins (cluster 8), while this was less observed in vivo. Previously we hypothesized that cyclosporin A induces ER stress, with an altered chaperone activity together with a disturbed protein transport resulting in a decreased protein secretion ${ }^{9}$. However, this reaction of cyclosporin $A$ seems characteristic for in vitro models. Possibly in vitro cell cultures are more sensitive because they are in direct contact with the toxicant and they function independent from other cells/organs.

Our analysis identified the similarities and differences in in vitro and in vivo models with respect to the response to cyclosporin A induced hepatotoxicity. Previous studies have shown 
the potential of the current in vitro models to detect drug-induced hepatotoxicit ${ }^{2,9,44}$. However the different toxicant-induced responses between in vivo and in vitro models explain the current difficulties to validate in vitro biomarkers towards in vivo models.

\section{ACKNOWLEDGMENTS}

We thank Erik Royackers from the Biomedical Research Institute of Hasselt University for his technical support of the LC-MS/MS analysis. The authors thank Piet Beekhof and Dr. Eugene Janssen for clinical chemistry analyses, and Joke Robinson and Dr. Leo van der Ven for histopathology analyses. 


\section{REFERENCES}

1 Kienhuis, A. S. et al. A sandwich-cultured rat hepatocyte system with increased metabolic competence evaluated by gene expression profiling. Toxicol In Vitro 21, 892-901 (2007).

2 Mathijs, K. et al. Assessing the metabolic competence of sandwich-cultured mouse primary hepatocytes. Drug Metab Dispos 37, 1305-1311 (2009).

3 Jennen, D. G. et al. Comparison of HepG2 and HepaRG by whole-genome gene expression analysis for the purpose of chemical hazard identification. Toxicol Sci 115, 66-79 (2010).

4 Kienhuis, A. S. et al. Parallelogram approach using rat-human in vitro and rat in vivo toxicogenomics predicts acetaminophen-induced hepatotoxicity in humans. Toxicol Sci 107, 544-552 (2009).

5 Heijne, W. H., Jonker, D., Stierum, R. H., van Ommen, B. \& Groten, J. P. Toxicogenomic analysis of gene expression changes in rat liver after a 28-day oral benzene exposure. Mutat Res 575, 85-101 (2005).

6 de Longueville, F. et al. Use of a low-density microarray for studying gene expression patterns induced by hepatotoxicants on primary cultures of rat hepatocytes. Toxicological sciences : an official journal of the Society of Toxicology 75, 378-392 (2003).

7 Greenbaum, D., Colangelo, C., Williams, K. \& Gerstein, M. Comparing protein abundance and mRNA expression levels on a genomic scale. Genome Biol 4, 117 (2003).

8 Minden, J. Comparative proteomics and difference gel electrophoresis. Biotechniques 43, 739, 741, 743 passim (2007)

9 Van Summeren, A. et al. Proteomics Investigations of Drug-Induced Hepatotoxicity in HepG2 Cells. Toxicol Sci 120, 109-122 (2011).

10 Van Summeren, A. et al. Screening for drug-induced hepatotoxicity in primary mouse hepatocytes using acetaminophen, amiodarone, and cyclosporin a as model compounds: an omics-guided approach. Omics : a journal of integrative biology 17, 71-83 (2013).

11 Rotolo, F. S., Branum, G. D., Bowers, B. A. \& Meyers, W. C. Effect of cyclosporine on bile secretion in rats. Am J Surg 151, 35-40 (1986).

12 Bouwman, F. G. et al. The Physiologic Effects of Caloric Restriction Are Reflected in the in Vivo AdipocyteEnriched Proteome of Overweight/Obese Subjects. J Proteome Res 8, 5532-5540 (2009).

13 Dumont, D., Noben, J. P., Raus, J., Stinissen, P. \& Robben, J. Proteomic analysis of cerebrospinal fluid from multiple sclerosis patients. Proteomics 4, 2117-2124 (2004).

14 Trauner, M. \& Boyer, J. L. Bile salt transporters: molecular characterization, function, and regulation. Physiol Rev 83, 633-671 (2003).

15 Alrefai, W. A. \& Gill, R. K. Bile acid transporters: structure, function, regulation and pathophysiological implications. Pharm Res 24, 1803-1823 (2007).

16 Kaplan, M. M. Primary biliary cirrhosis--a first step in prolonging survival. N Engl J Med 330, 1386-1387 (1994).

17 Wolf, A. et al. Cyclosporine A-induced oxidative stress in rat hepatocytes. J Pharmacol Exp Ther 280, 1328-1334 (1997)

18 Jimenez, R., Galan, A. I., Gonzalez de Buitrago, J. M., Palomero, J. \& Munoz, M. E. Glutathione metabolism in cyclosporine A-treated rats: dose- and time-related changes in liver and kidney. Clin Exp Pharmacol Physiol 27, 991-996 (2000).

19 Spivey, J. R., Bronk, S. F. \& Gores, G. J. Glycochenodeoxycholate-induced lethal hepatocellular injury in rat hepatocytes. Role of ATP depletion and cytosolic free calcium. J Clin Invest 92, 17-24 (1993).

20 Gores, G. J., Miyoshi, H., Botla, R., Aguilar, H. I. \& Bronk, S. F. Induction of the mitochondrial permeability transition as a mechanism of liver injury during cholestasis: a potential role for mitochondrial proteases. Biochim Biophys Acta 1366, 167-175 (1998).

21 Gomes Martins, E., Santos Silva, E., Vilarinho, S., Saudubray, J. M. \& Vilarinho, L. Neonatal cholestasis: an uncommon presentation of hyperargininemia. J Inherit Metab Dis (2011).

22 Tazawa, Y. et al. A possible mechanism of neonatal intrahepatic cholestasis caused by citrin deficiency. Hepatol Res 31, 168-171 (2005).

23 Moyer, V. et al. Guideline for the evaluation of cholestatic jaundice in infants: recommendations of the North American Society for Pediatric Gastroenterology, Hepatology and Nutrition. J Pediatr Gastroenterol Nutr 39, 115 128 (2004). 
24 Lohr, J. W., Willsky, G. R. \& Acara, M. A. Renal drug metabolism. Pharmacol Rev 50, 107-141 (1998).

25 Galan, A. I., Munoz, M. E. \& Jimenez, R. S-Adenosylmethionine protects against cyclosporin A-induced alterations in rat liver plasma membrane fluidity and functions. J Pharmacol Exp Ther 290, 774-781 (1999).

26 Fernandez, E. et al. Reversal of cyclosporine A-induced alterations in biliary secretion by S-adenosyl-L-methionine in rats. J Pharmacol Exp Ther 275, $442-449$ (1995).

27 Friedel, H. A., Goa, K. L. \& Benfield, P. S-adenosyl-L-methionine. A review of its pharmacological properties and therapeutic potential in liver dysfunction and affective disorders in relation to its physiological role in cell metabolism. Drugs 38, 389-416 (1989).

28 Boelsterli, U. A., Rakhit, G. \& Balazs, T. Modulation by S-adenosyl-L-methionine of hepatic Na+,K+-ATPase, membrane fluidity, and bile flow in rats with ethinyl estradiol-induced cholestasis. Hepatology 3, 12-17 (1983).

$29 \mathrm{Kim}, \mathrm{Y}$. H. \& Joo, 2nd. Arylamine N-methyltransferase and thiol methyltransferase activities in cholestatic rat liver induced by common bile duct ligation. Exp Mol Med 33, 23-28 (2001).

30 Tiao, M. M., Lin, T. K., Wang, P. W., Chen, J. B. \& Liou, C. W. The role of mitochondria in cholestatic liver injury. Chang Gung Med J 32, 346-353 (2009).

31 Vendemiale, G., Grattagliano, I., Lupo, L., Memeo, V. \& Altomare, E. Hepatic oxidative alterations in patients with extra-hepatic cholestasis. Effect of surgical drainage. J Hepatol 37, 601-605 (2002).

32 Agellon, L. B. \& Torchia, E. C. Intracellular transport of bile acids. Biochim Biophys Acta 1486, 198-209 (2000).

33 Chisholm, J. W., Nation, P., Dolphin, P. J. \& Agellon, L. B. High plasma cholesterol in drug-induced cholestasis is associated with enhanced hepatic cholesterol synthesis. Am J Physiol 276, G1165-1173 (1999).

34 Kienhuis, A. S. et al. Phenotype-directed gene expression analysis of CSA induced cholestasis in C57BL/6J mice for comparison of in vivo to in vitro models of hepatotoxicity. (2013).

35 Ferre, N., Camps, J., Cabre, M., Paul, A. \& Joven, J. Hepatic paraoxonase activity alterations and free radical production in rats with experimental cirrhosis. Metabolism 50, 997-1000 (2001).

36 Amacher, D. E., Adler, R., Herath, A. \& Townsend, R. R. Use of proteomic methods to identify serum biomarkers associated with rat liver toxicity or hypertrophy. Clin Chem $\mathbf{5 1}$, 1796-1803 (2005).

37 Adler, M. et al. Assessment of candidate biomarkers of drug-induced hepatobiliary injury in preclinical toxicity studies. Toxicol Lett 196, 1-11 (2010).

38 Jaeschke, H., McGill, M. R. \& Ramachandran, A. Oxidant stress, mitochondria, and cell death mechanisms in druginduced liver injury: Lessons learned from acetaminophen hepatotoxicity. Drug Metab Rev 44, 88-106 (2012).

39 Wang, P., Bouwman, F. G. \& Mariman, E. C. Generally detected proteins in comparative proteomics--a matter of cellular stress response? Proteomics 9, 2955-2966 (2009).

40 Boess, F. et al. Gene expression in two hepatic cell lines, cultured primary hepatocytes, and liver slices compared to the in vivo liver gene expression in rats: possible implications for toxicogenomics use of in vitro systems. Toxicol Sci 73, 386-402 (2003).

41 Wilkening, S. \& Bader, A. Influence of culture time on the expression of drug-metabolizing enzymes in primary human hepatocytes and hepatoma cell line HepG2. J Biochem Mol Toxicol 17, 207-213 (2003).

42 Carpenter, B. et al. The roles of heterogeneous nuclear ribonucleoproteins in tumour development and progression. Biochim Biophys Acta 1765, 85-100 (2006).

43 Lenaerts, K., Bouwman, F. G., Lamers, W. H., Renes, J. \& Mariman, E. C. Comparative proteomic analysis of cell lines and scrapings of the human intestinal epithelium. BMC Genomics 8, 91 (2007).

44 Wang, K., Shindoh, H., Inoue, T. \& Horii, I. Advantages of in vitro cytotoxicity testing by using primary rat hepatocytes in comparison with established cell lines. J Toxicol Sci 27, 229-237 (2002). 


\section{CHAPTER 7}

\section{Analysis of protein expression patterns induced by classifying hepatotoxicants in primary mouse hepatocytes}

Anke Van Summeren, Johan Renes, Freek G. Bouwman, Jean-Paul Noben, Joost H. M. van Delft, Jos C. S. Kleinjans and Edwin C. M. Mariman 


\section{ABSTRACT}

The assessment of potential hepatotoxic compounds remains a challenge in the development of novel drugs. The currently used chronic rodent bioassays have only a limited reliability when it comes to predicting human hepatotoxicity. In addition they are time-consuming and have ethical drawbacks. Drug-induced liver failure comprises several phenotypes including necrosis, cholestasis and steatosis. The use of proteomics in an in vitro system may provide an alternative for the evaluation of hepatotoxcity.

In this study, we investigated whether compounds inducing similar toxicological endpoints in the liver produce similar changes in protein expression in an in vitro system. Primary mouse hepatocytes were exposed to six well-characterized hepatotoxicants selected based on their different hepatotoxic phenotypes: diclofenac and paraquat (necrosis), tetracycline and valproic acid (steatosis), chlorpramazine and ethinylestradiol (cholestasis), and two nephrotoxicants as negative controls: D-mannitol and $\mathrm{LiCO}_{3}$. Afterwards, changes in protein expression were determined by means of difference gel electrophoresis. By using a discriminant principal component analysis we attempted to differentiate between the hepatotoxic phenotypes. For this analysis we have included the protein spot maps from our previous study where primary mouse hepatocytes were exposed to acetaminophen, amiodarone and cyclosporin $A$, inducing necrosis, steatosis and cholestasis, respectively. From the discriminant principal component analysis the necrotizing compounds were mainly discriminated because of differential expression of Cat, Gpt2, Ugp2, Arg1 and Prdx6. Compounds which induce cholestasis could be distinguished on the basis of the expression of $\mathrm{Gc}$ and compounds which induce steatosis were distinguished by means of Cpt2. However, the classification of the different hepatotoxic phenotypes is challenged by the overlap of some phenotypical characteristics of the different hepatotoxic classes.

The proteome of the primary mouse hepatocytes reflected several hepatotoxic mechanisms related to the different phenotypes. Furthermore, the nephrotoxic compounds used as negative controls did not induce any hepatoxic effects, indicating the specific response of primary mouse hepatocytes to hepatotoxicants. 


\section{INTRODUCTION}

One of the main concerns in the development of novel drugs, is the withdrawal of potential new drug candidates due to undesired toxicity during preclinical studies and clinical trials ${ }^{1}$. When it comes to drug-induced toxicity, liver injury is the most prominent. This is because the liver is responsible for the detoxification and elimination of toxic compounds. The current tests to identify organ toxicity often rely on chronic rodent bioassays for traditional biological endpoints. These assays are relatively insensitive and generate false negative results ${ }^{2,3}$. Furthermore, it may take weeks, months, or even years before some of these traditional toxicological endpoints become manifest. On top of these limitations, chronic rodent assays are expensive, time consuming, and connected with ethical drawbacks. This emphasize the need for new preclinical screening methods to detect toxicological hazards earlier in the drug discovery process. Presently, hepatic in vitro models have shown promising results in toxicological research. Especially the use of primary hepatocytes is preferred, because of their liver specific characteristics like the expression of biotransformation enzymes. While, primary rat and human primary hepatocytes are well-described in vitro models, studies describing the use of primary mouse hepatocytes are limited. However, mouse models are gaining interest in toxicological studies thanks to the availability of transgenic mouse models. These models can provide primary hepatocytes suitable for dedicated mechanistic investigations of liver toxicity ${ }^{4}$. Furthermore, primary mouse hepatocytes maintain their metabolic competence better compared to primary rat hepatocytes ${ }^{5}$.

Drug-induced hepatotoxicity includes several phenotypes including necrosis, cholestasis and steatosis. Excessive injury to mitochondria caused by free radicals and/or toxic metabolites can cause necrosis, which is a form of premature cell death due to external factors ${ }^{6}$. Cholestasis is caused by impairment of the bile-salt transporter proteins leading to accumulation of bile, causing secondary injury to hepatocytes ${ }^{7}$. Steatosis is caused by a disturbed $\beta$-oxidation of lipids. Interruption of the $\beta$-oxidation cycle in hepatocytes will lead to intracellular accumulation of small lipid vesicles ${ }^{8}$. Besides the identification of hepatotoxic compounds it is preferable to classify these compounds based on their phenotypic toxicity pathway ${ }^{9}$.

Previously, it was shown that compounds from a similar toxicity class induced similar gene expression profiles in hepatic in vitro liver models, which could be discriminated from the profiles generated by a different class of chemicals ${ }^{10-13}$. These studies indicate that expression profiling applying 'omics technologies to organic cellular models have the potential to improve current toxicity tests ${ }^{14}$. Nowadays, transcriptomics is still the most frequently used technology within the field of toxicogenomics. Microarrays allow the simultaneous measurement of thousands of mRNA transcripts for gene expression. However, it is acknowledged that the relative gene expression levels often only moderately correlate with the protein expression. By means of proteomics techniques it is possible to generate protein expression profiles for drug-induced hepatotoxicity ${ }^{15-18}$. Furthermore, proteomics data correlates well 
with the clinical and histological data ${ }^{19}$. In addition, proteomic changes can even occur before the onset of toxicity ${ }^{19}$.

In this study, we investigated whether compounds inducing similar toxicological endpoints produce similar changes in protein expression. Primary mouse hepatocytes were exposed to six well-characterized hepatotoxicants selected on their different hepatotoxic phenotypes: diclofenac and paraquat (necrosis), tetracycline and valproic acid (steatosis), chlorpramazine and ethinylestradiol (cholestasis), and two nephrotoxicants, D-mannitol and $\mathrm{LiCO}_{3}$, as negative controls. Afterwards, differentially expressed proteins were analyzed by means of difference gel electrophoresis (DIGE).

In addition, we included the protein spot maps fom our previous study where primary mouse hepatocytes were exposed to acetaminophen, amiodarone and cyclosporin A, inducing respectively necrosis, steatosis and cholestasis.

\section{MATERIALS AND METHODS}

\section{Chemicals}

Dulbecco's modified Eagle's medium, fetal calf serum, penicillin/streptomycin, Hanks' calcium- and magnesium-free buffer and insulin were obtained from Life Technologies Europe BV (Bleiswijk, The Netherlands). Glucagon, hydrocortisone (water soluble), collagenase type IV, Dimethylsulfoxide (DMSO), Trypan blue, 3-(4,5-dimethylthiazol-2-yl)-2,5-diphenyltetrazolium bromide, $\mathrm{NaCl}, \mathrm{NaHCO}_{3}, \mathrm{KCl} ; \mathrm{KH}_{2} \mathrm{PO}_{4} ; \mathrm{MgSO}_{4}$; glucose; $\mathrm{CaCl}_{2}$, chlorpramazine, tetracycline, ethinylestradiol, valproic acid, diclofenac, paraquat, D-mannitol, $\mathrm{LiCO}_{3}$ and $\mathrm{N}, \mathrm{N}$ dimethylformamide (anhydrous, $99.8 \%$ ) were purchased from Sigma-Aldrich (Zwijndrecht, The Netherlands). Collagen Type I Rat Tail was obtained from BD BioSciences (Bedford, MA, USA), the Protein Assay Kit was from Bio-Rad (Veenendaal, The Netherlands). All chemicals used for DIGE were purchased from GE Healthcare (Diegem, Belgium).

\section{Animals}

Permission for animal studies was obtained from the Animal Ethical Committee, from the Maastricht University, The Netherlands. Adult male C57/B6 mice, weighing 20-25 g, were obtained from Charles River $\mathrm{GmbH}$ (Sulzfeld, Germany). The animals were housed in macrolon cages with sawdust bedding at $22^{\circ} \mathrm{C}$ and 50-60\% humidity. The light cycle was $12 \mathrm{~h}$ light/12 $\mathrm{h}$ dark. Food and tap water were available ad libitum.

\section{Isolation and culture of primary mouse hepatocytes}

Hepatocytes were isolated by a two-step collagenase perfusion method according to Seglen ${ }^{20}$, with modifications as described before ${ }^{5}$. 
Cell suspensions with cell viability $\geq 80 \%$, determined by trypan blue exclusion, were brought into culture in a collagen-collagen sandwich formation as described before ${ }^{5}$. Prior to treatment, the primary mouse hepatocytes were allowed to recover for $40-42 \mathrm{~h}$ at $37^{\circ} \mathrm{C}$ in a humidified chamber with $95 \%$ / 5 \% air / CO2 in serum-free culture medium supplemented with insulin $(0.5 \mathrm{U} / \mathrm{ml})$, glucagon $(7 \mathrm{ng} / \mathrm{ml})$, hydrocortisone $(7.5 \mu \mathrm{g} / \mathrm{ml})$, and $2 \%$ penicillin/streptomycin $(5000 \mathrm{U} / \mathrm{ml}$ penicillin and $5000 \mathrm{Im} / \mathrm{ml}$ streptomycin). Culture medium was refreshed every $24 \mathrm{~h}$. After the recovery period, the culture medium was replaced by culture medium containing one of the 8 selected compounds or with the vehicle controls $0.5 \%$ PBS and DMSO. For each compound the $\mathrm{IC}_{20}$ concentration after $48 \mathrm{~h}$ of exposure as determined by MTT reduction method ${ }^{21}$ was used. The compounds and their applied concentrations are shown in Table 7.1. This table also includes concentrations of hepatotoxicants applied in a previous study ${ }^{22}$, from which the data is included here for a comparative analysis. Each test condition consists of five independent biological experiments, each with cells from a different animal.

\section{Clinical chemistry}

The medium of the primary mouse hepatocytes was used to analyze Aspartate aminotransferase (AST), Alanine aminotransferase (ALT), Lactate dehydrogenase (LD) and Total Bilirubin (TBIL) on a Beckman Coulter LX20 Clinical Chemistry Analyzer using Beckman reagent kits (Beckman Coulter B.V., Woerden, The Netherlands).

\section{Protein extraction}

The cells were washed twice with PBS. For protein extraction from the hepatocytes the collagen layers were removed to prevent its interference with the proteome analysis. Intact cells were isolated from the collagen layers after $10 \mathrm{~min}$ incubation with collagenase buffer. The collagenase buffer contains $1150 \mathrm{CDU} / 100 \mathrm{ml}$ collagenase (CDU = collagen digestion units) dissolved in a Krebs buffer (118 mM NaCl; $25 \mathrm{mM} \mathrm{NaHCO}_{3} ; 4.8 \mathrm{mM} \mathrm{KCl} ; 1.2 \mathrm{mM} \mathrm{KH}_{2} \mathrm{PO}_{4} ; 1.2$ $\mathrm{mM} \mathrm{MgSO}_{4} ; 11 \mathrm{mM}$ glucose; $1.5 \mathrm{mM} \mathrm{CaCl}_{2}$ ). The suspension of detached cells was washed and further diluted till $50 \mathrm{ml}$ with PBS, and centrifuged for $5 \mathrm{~min}$ at $65 \mathrm{~g}$. To wash the cells thoroughly, the washing step was repeated. Afterwards the supernatant was removed and the cell pellet was dissolved in a DIGE labeling buffer containing $7 \mathrm{M}$ urea, $2 \mathrm{M}$ thiourea, $4 \%(w / v)$ CHAPS and $30 \mathrm{mM}$ Tris- $\mathrm{HCl}$. This mixture was subjected to three cycles of freeze thawing with liquid nitrogen, vortexed thoroughly and centrifuged at $20000 \mathrm{~g}$ for $30 \mathrm{~min}$ at $10^{\circ} \mathrm{C}$. Supernatant was collected, aliquoted and stored at $-80^{\circ} \mathrm{C}$ until further analysis. Protein concentrations were determined with the Protein Assay Kit from Bio-Rad (Veenendaal, The Netherlands). 
Table 7.1: Hepatotoxic compounds with their applied concentrations, solvent and the hepatotoxic phenotype they induce.

\begin{tabular}{|c|c|c|c|}
\hline Compound & Dose & $\begin{array}{l}\text { Solvent } \\
\text { (dose v/v (\%)) }\end{array}$ & $\begin{array}{l}\text { Hepatotoxicity } \\
\text { class }\end{array}$ \\
\hline Tetracycline & $200 \mu \mathrm{M}$ & DMSO (0.5 \%) & Steatosis \\
\hline Valproic acid & $10 \mathrm{mM}$ & PBS (0.5 \%) & Steatosis \\
\hline Amiodarone ${ }^{*}$ & $1 \mu \mathrm{M}$ & DMSO (0.5 \%) & Steatosis \\
\hline Ethinylestradiol & $100 \mu \mathrm{M}$ & DMSO (0.5 \%) & Cholestasis \\
\hline Chlorpramazine & $20 \mu \mathrm{M}$ & DMSO (0.5 \%) & Cholestasis \\
\hline Cyclosporin $\mathrm{A}^{*}$ & $10 \mu \mathrm{M}$ & DMSO (0.5 \%) & Cholestasis \\
\hline Diclofenac & $600 \mu \mathrm{M}$ & DMSO (0.5 \%) & Necrosis \\
\hline Paraquat & $15 \mu \mathrm{M}$ & PBS (0.5 \%) & Necrosis \\
\hline Acetaminophen ${ }^{*}$ & $1 \mathrm{mM}$ & DMSO (0.5 \%) & Necrosis \\
\hline D-mannitol & $2 \mathrm{mM}$ & DMSO (0.5 \%) & Non-hepatotoxic \\
\hline $\mathrm{LiCO}_{3}$ & $0.5 \mathrm{mM}$ & PBS (0.5 \%) & Non-hepatotoxic \\
\hline
\end{tabular}

${ }^{*}$ These compounds were used in chapter $3^{22}$.

\section{Difference gel electrophoresis}

The protein labeling and the DIGE were performed as described before ${ }^{18}$.

A one-way ANOVA test $(P \leq 0.05)$ was used to select the significant differential spots between the experimental groups. The differentially expressed proteins were excised and identified by matrix assisted laser desorption ionization time-of-flight (MALDI-TOF/TOF) mass spectrometry 23. Protein spots that could not be identified via MALDI-TOF MS were further analyzed by nano liquid chromatography tandem mass spectrometry (nLC-MS/MS) on an LCQ Classic (ThermoFinnigan) as described ${ }^{24}$.

\section{Discriminant analysis of principal components}

The spot maps from this experiment were matched with the spot maps from previous experiments ${ }^{22}$. The spot intensities were exported from the Decyder software and imported into the GeneMath XT software, version 2.12 (Applied Maths, Sint-Martens-Latem, Belgium). The spot maps of the compounds were grouped according to their drug class (Table 7.1), afterwards a supervised discriminant principal component analysis was performed. The experiment with diclofenac was considered as an outlier because all the spotmaps from diclofenac were positioned outside the PCA model and therefore excluded from the analysis. A variable with a geometric mean of the loading score on all components $>2.7$ was taken as an important contributor. 
A

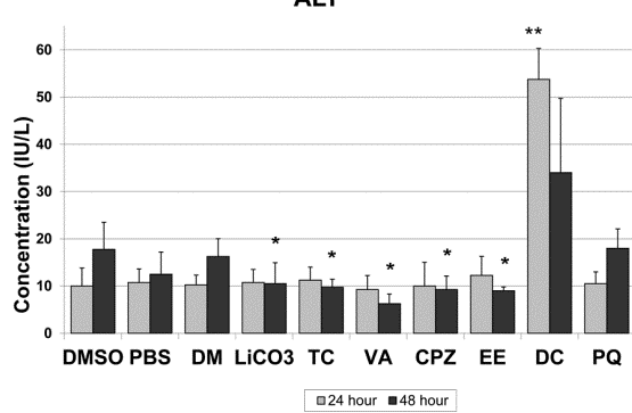

B

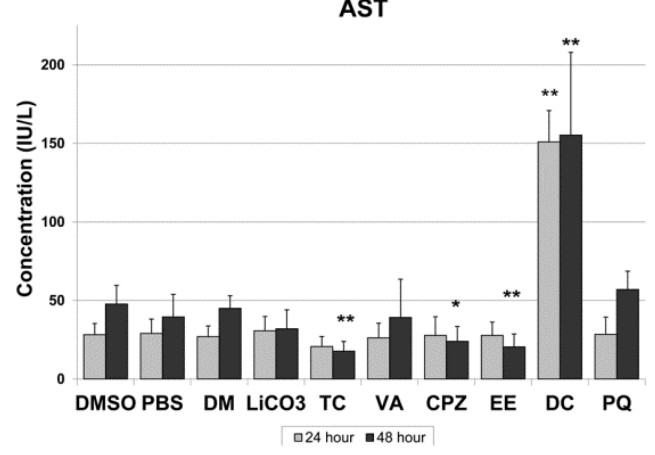

C

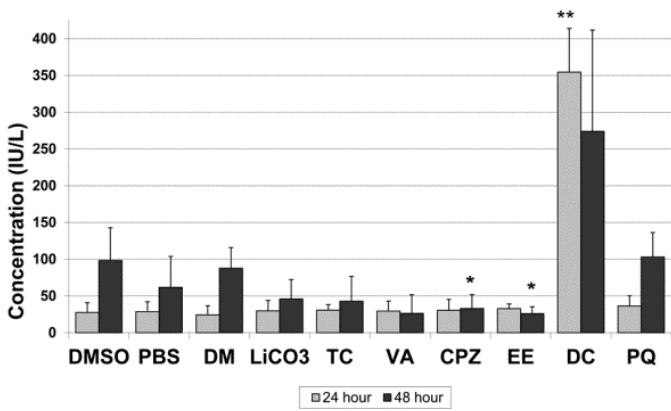

D

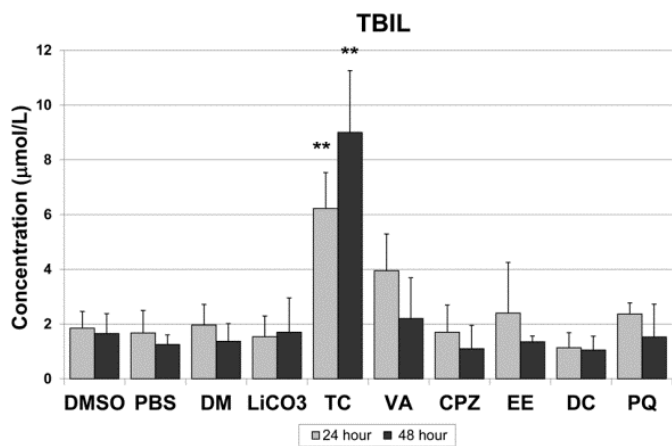

Figure 7.1: average values of (A) ALT ; (B) AST, (C) LD and (D) TBIL measured in the cell culture medium of the primary mouse hepatocytes. ${ }^{* *}$ indicates significant values in a student T-test with a P-value $\leq 0.01 .{ }^{*}$ indicates significant values with a P-value $\leq 0.05$ compared with the vehicle control, calculated with a student T-test.

\section{RESULTS}

\section{Clinical parameters}

In the medium of the primary mouse hepatocytes we measured ALT, AST, LD and TBIL (Figure 7.1). In comparison with the control samples and the other compounds, diclofenac significantly induced ALT, AST and LD. High levels of these metabolic enzymes also indicate an increased amount of cell death despite the application of an $\mathrm{IC}_{20}$ concentration.

Surprisingly, tetracycline significantly increased TBIL levels in the medium of the primary mouse hepatocytes. In addition, valproic acid showed a trend for increased TBIL levels in the medium of primary mouse hepatocytes. Increased TBIL levels are usually associated with cholestasis and together with AST it is the most important predictor of drug-induced hepatotoxicity ${ }^{25}$. 


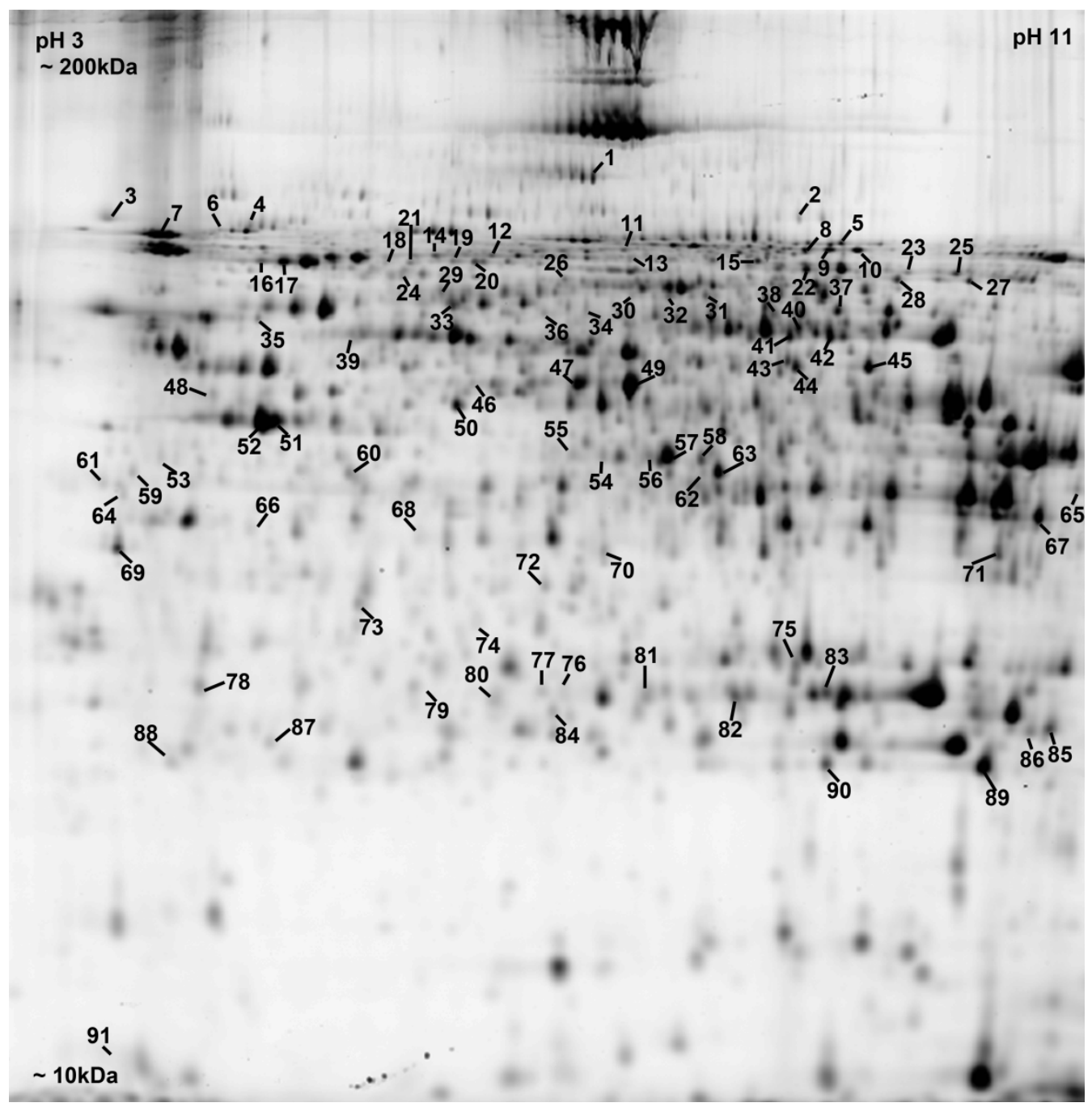

Figure 7.2: Proteome map of the differentially expressed proteins. The identified spots are indicated with a number which corresponds to the numbers used in Supplemental Table 7.1.

\section{DIGE analysis}

The cellular proteins of the treated primary mouse hepatocytes were analyzed using DIGE. In total 3125 spots could be matched with all the images. With a one-way ANOVA analysis ( $\mathrm{P} \leq$ $0.05) 418$ spots were detected as significantly differential. Based on a multiple comparison test 290 of the differentially expressed protein spots were assigned to diclofenac. Probably these differences are mainly caused by cell damage, since the clinical parameters showed high levels of AST, ALT and LD in the cell culture medium after exposure to diclofenac. Be- 
cause the aim of the study is to distinguish the differentially expressed proteins between drug-induced steatosis, cholestasis and necrosis before the onset of cell death, the spot maps from the diclofenac-treated hepatocytes were excluded from the analysis. After removal of the diclofenac-treated samples, 128 spots were detected as differentially expressed with a one-way ANOVA analysis $(\mathrm{P} \leq 0.05)$.

\section{Protein identification from the differential spots}

The differential spots were included in a pick list and excised from a preparative gel. Protein identification was performed by in-gel digestion followed by MALDI-TOF/TOF and/or nLCMS/MS analysis. Out of the 128 spots, the proteins of 91 spots were identified which belonged to 64 different proteins (Supplemental Table 7.1). Figure 7.2 shows the 2-DE map made from the master gel with the 91 identified differential spots indicated with a number that corresponds to the numbers presented in Supplemental Table 7.1.

Tables 7.2 to 7.4 lists the differentially expressed proteins according to the hepatotoxic phenotypes. Twenty-eight spots appeared isoforms from 16 proteins due to post-translational modifications or processing of the protein. For spot No. 76 two proteins were identified with $\mathrm{nLC}-\mathrm{MS} / \mathrm{MS}$, both protein identifications delivered the same number of peptides. Consequently, for spot No. 76 it is not possible to conclude which protein is responsible for the significant change.

Table 7.2: Protein identification of differentially expressed proteins in primary mouse hepatocytes after exposure to the steatosis-inducers tetracycline and valproic acid.

\begin{tabular}{|c|c|c|c|c|c|c|}
\hline \multirow{2}{*}{ No. } & \multirow{2}{*}{$\begin{array}{c}\text { Uniprot } \\
\text { Accession } \\
\text { No. }\end{array}$} & \multirow[b]{2}{*}{$\begin{array}{l}\text { Gene } \\
\text { name }\end{array}$} & \multirow[b]{2}{*}{ Protein description } & \multirow{2}{*}{$\begin{array}{l}\text { P-value } \\
\text { (one-way } \\
\text { ANOVA) }^{1}\end{array}$} & \multicolumn{2}{|c|}{ Fold Change $^{2}$} \\
\hline & & & & & $\begin{array}{l}\mathrm{DMSO} / \mathrm{T} \\
\mathrm{C}\end{array}$ & $\begin{array}{l}\text { PBS/ } \\
\text { VA }\end{array}$ \\
\hline 2 & Q78PY7 & Snd1 & $\begin{array}{l}\text { Staphylococcal nuclease domain- } \\
\text { containing protein } 1\end{array}$ & 0.0042 & 1.06 & $1.39^{*}$ \\
\hline 3 & P08113 & Hsp90b1 & Endoplasmin & 0.00024 & 1.03 & 1.49 \\
\hline 11 & Q8CAQ8 & Immt & Mitochondrial inner membrane protein & 0.033 & -1.03 & $1.32^{*}$ \\
\hline 12 & Q9CZD3 & Gars & Glycyl-tRNA synthetase & $3.90 \mathrm{E}-06$ & -1 & $1.42^{*}$ \\
\hline 15 & P41216 & Acsl1 & Long-chain-fatty-acid--CoA ligase 1 & 0.00043 & -1.12 & $1.1^{*}$ \\
\hline 20 & P07724 & Alb & Serum albumin & 1.60E-05 & $1.06^{*}$ & $-1.1^{*}$ \\
\hline 21 & P07724 & Alb & Serum albumin & 0.00022 & $-1.11^{*}$ & 1.03 \\
\hline 23 & Q9Z0X1 & Aifm1 & Apoptosis-inducing factor 1 & 0.008 & -1.07 & $-1.05^{*}$ \\
\hline 25 & Q9Z0X1 & Aifm1 & $\begin{array}{l}\text { Apoptosis-inducing factor } 1 \text {, mitochon- } \\
\text { drial }\end{array}$ & $3.90 \mathrm{E}-06$ & -1.1 & $-1.06^{*}$ \\
\hline 27 & P52825 & Cpt2 & $\begin{array}{l}\text { Carnitine O-palmitoyltransferase 2, } \\
\text { mitochondrial }\end{array}$ & 0.0079 & 1.19 & $1.13^{*}$ \\
\hline 28 & P50544 & Acadvl & $\begin{array}{l}\text { Very long-chain specific acyl-CoA dehy- } \\
\text { drogenase, mitochondrial }\end{array}$ & $3.90 \mathrm{E}-05$ & 1.01 & $1.31^{*}$ \\
\hline 30 & Q8VCW8 & Acsf2 & $\begin{array}{l}\text { Acyl-CoA synthetase family member } 2 \text {, } \\
\text { mitochondrial }\end{array}$ & 0.0047 & 1.11 & -1.09 \\
\hline 31 & Q8VCW8 & Acsf2 & $\begin{array}{l}\text { Acyl-CoA synthetase family member } 2 \text {, } \\
\text { mitochondrial }\end{array}$ & $9.60 \mathrm{E}-07$ & 1.11 & $-1.02^{*}$ \\
\hline 32 & Q8VCW8 & Acsf2 & $\begin{array}{l}\text { Acyl-CoA synthetase family member } 2 \\
\text { mitochondrial }\end{array}$ & $3.50 \mathrm{E}-05$ & 1.01 & $-1.54^{*}$ \\
\hline
\end{tabular}




\begin{tabular}{|c|c|c|c|c|c|c|}
\hline \multirow{2}{*}{ No. } & \multirow{2}{*}{$\begin{array}{c}\text { Uniprot } \\
\text { Accession } \\
\text { No. }\end{array}$} & \multirow[b]{2}{*}{$\begin{array}{l}\text { Gene } \\
\text { name }\end{array}$} & \multirow[b]{2}{*}{ Protein description } & \multirow{2}{*}{$\begin{array}{l}\text { P-value } \\
\text { (one-way } \\
\text { ANOVA) }\end{array}$} & \multicolumn{2}{|c|}{ Fold Change $^{2}$} \\
\hline & & & & & $\begin{array}{l}\mathrm{DMSO} / \mathrm{T} \\
\mathrm{C}\end{array}$ & $\begin{array}{l}\text { PBS/ } \\
\text { VA }\end{array}$ \\
\hline 33 & P27773 & Pdia3 & Protein disulfide-isomerase $\mathrm{A} 3$ & 0.0024 & 1.1 & $-1.4^{*}$ \\
\hline 34 & Q00612 & G6pdx & $\begin{array}{l}\text { Glucose-6-phosphate 1-dehydrogenase } \\
X\end{array}$ & $3.90 \mathrm{E}-05$ & 1.12 & $-1.3^{*}$ \\
\hline 35 & P21614 & Gc & Vitamin D-binding protein & 0.0049 & 1 & $-1.07^{*}$ \\
\hline 38 & P24270 & Cat & Catalase & 0.0028 & -1.09 & $-1.27^{*}$ \\
\hline 40 & Q8BGT5 & Gpt2 & Alanine aminotransferase 2 & 0.0049 & -1 & -1.41 \\
\hline 42 & Q91ZJ5 & Ugp2 & $\begin{array}{l}\text { UTP--glucose-1-phosphate uridylyl- } \\
\text { transferase }\end{array}$ & 0.0065 & -1.18 & $-1.33^{*}$ \\
\hline 44 & P54869 & Hmgcs2 & $\begin{array}{l}\text { Hydroxymethylglutaryl-CoA synthase, } \\
\text { mitochondrial }\end{array}$ & 0.007 & 1.07 & $-1.3^{*}$ \\
\hline 45 & P51855 & Gss & Glutathione synthetase & 0.0085 & -1.06 & -1.13 \\
\hline 48 & P05784 & Krt18 & Keratin, type I cytoskeletal 18 & 0.0026 & -1.04 & $-1.64^{*}$ \\
\hline 49 & P17182 & Eno1 & Alpha-enolase & 0.0007 & -1.19 & -1.07 \\
\hline 50 & P55264 & Adk & Adenosine kinase & 0.0028 & $-1.2^{*}$ & -1.05 \\
\hline 51 & P60710 & Actb & Actin, cytoplasmic 1 & 0.032 & -1.17 & $-1.35^{*}$ \\
\hline 52 & P60710 & Actb & Actin, cytoplasmic 1 & 0.00036 & -1.12 & $1.33^{*}$ \\
\hline 53 & P11679 & Krt8 & Keratin, type II cytoskeletal 8 & 0.05 & -1.31 & -2.46 \\
\hline 59 & P11679 & Krt8 & Keratin, type II cytoskeletal 8 & 0.00069 & -1.75 & $-2.75^{*}$ \\
\hline 60 & P60710 & Actb & Actin, cytoplasmic 1 & 0.00066 & -1.83 & $-2.02^{*}$ \\
\hline 62 & Q61176 & Arg1 & Arginase-1 & $6.00 \mathrm{E}-07$ & -1.08 & $1.23^{*}$ \\
\hline 64 & P05784 & Krt18 & Keratin, type I cytoskeletal 18 & 0.0011 & -1.94 & $-2.7^{*}$ \\
\hline 67 & P08249 & Mdh2 & Malate dehydrogenase, mitochondrial & 0.0022 & 1.02 & $1.36^{*}$ \\
\hline 71 & Q60932 & Vdac1 & $\begin{array}{l}\text { Voltage-dependent anion-selective } \\
\text { channel protein } 1\end{array}$ & 0.023 & 1.19 & $1.46^{*}$ \\
\hline 74 & Q99P30 & Nudt7 & $\begin{array}{l}\text { Isoform } 1 \text { of Peroxisomal coenzyme A } \\
\text { diphosphatase NUDT7 }\end{array}$ & 0.014 & $1.43^{*}$ & -1.04 \\
\hline 77 & 008807 & Prdx4 & Peroxiredoxin-4 & 0.00046 & -1.16 & $-1.37^{*}$ \\
\hline 88 & Q61171 & $\operatorname{Prdx} 2$ & Peroxiredoxin-2 & 0.0084 & $-1.21^{*}$ & 1.03 \\
\hline 89 & P35700 & Prdx1 & Peroxiredoxin-1 & $1.60 \mathrm{E}-06$ & -1.05 & $1.25^{*}$ \\
\hline
\end{tabular}

\footnotetext{
${ }^{1}$ P-value from one way ANOVA statistical test between the experimental groups (paraquat, tetracycline, valproic acid, chlorpromazine, ethinylestradiol, negative controls D-mannitol and $\mathrm{LiCO}_{3}$ and the vehicle controls $\mathrm{DMSO}$ and PBS) with each five biological replicates.

${ }^{2}$ The difference in the standardized abundance of the proteins is expressed as the fold change between the control (C) and the treated groups (T). The fold change is calculated by taking the means of standardized volume values for the protein spot in the corresponding groups (PBS = PBS vehicle control, DMSO = DMSO vehicle control, $\mathrm{TC}=$ tetracycline and VA = valproic acid), values are calculated as T/C and displayed in the range of +1 to $+\infty$ for increases in expression and calculated as $-\mathrm{C} / \mathrm{T}$ and displayed in the range of $-\infty$ to -1 for decreased expression.

* Indicates significant fold changes $(\mathrm{P} \leq 0.05)$ between the control and the treated group, calculated with a multiple comparison test.
} 
Table 7.3: Protein identification of differentially expressed proteins in primary mouse hepatocytes after exposure to the necrosis-inducer paraquat.

\begin{tabular}{|c|c|c|c|c|c|}
\hline No & $\begin{array}{c}\text { Uniprot } \\
\text { Accession } \\
\text { No }\end{array}$ & $\begin{array}{l}\text { Gene } \\
\text { name }\end{array}$ & Protein description & $\begin{array}{c}\text { P-value } \\
\text { (one-way } \\
\text { ANOVA) }^{1}\end{array}$ & $\begin{array}{c}\text { Fold } \\
\text { Change }^{2} \\
\text { PBS/PQ }\end{array}$ \\
\hline 5 & P28271 & Aco1 & Iron-responsive element binding protein 1 & $2.40 \mathrm{E}-07$ & $-1.06^{*}$ \\
\hline 10 & Q99KIO & Aco2 & Aconitate hydratase, mitochondrial precursor & $5.60 \mathrm{E}-06$ & $-1.2^{*}$ \\
\hline 14 & P97494 & Gclc & Glutamate--cysteine ligase catalytic subunit & 0.0031 & 1.06 \\
\hline 16 & P63017 & Hspa8 & Heat shock cognate $71 \mathrm{kDa}$ protein & 0.0054 & 1.1 \\
\hline 21 & P07724 & Alb & Serum albumin & 0.00022 & -1 \\
\hline 36 & Q9JMH6 & Txnrd1 & $\begin{array}{l}\text { Isoform } 2 \text { of Thioredoxin reductase } 1 \text {, cytoplas- } \\
\text { mic }\end{array}$ & 0.013 & 1.37 \\
\hline 37 & P24270 & Cat & Catalase & 0.002 & $1.29^{*}$ \\
\hline 43 & 009173 & Hgd & Homogentisate 1,2-dioxygenase & 0.0019 & $-1.26^{*}$ \\
\hline 46 & P46471 & Psmc2 & $26 S$ protease regulatory subunit 7 & 0.0084 & $1.46^{*}$ \\
\hline 54 & Q07417 & Acads & $\begin{array}{l}\text { Short-chain specific acyl-CoA dehydrogenase, } \\
\text { mitochondrial }\end{array}$ & 0.019 & 1.25 \\
\hline 56 & Q61176 & $\operatorname{Arg} 1$ & Arginase-1 & 3.10E-05 & $1.53^{*}$ \\
\hline 58 & Q64442 & Sord & Sorbitol dehydrogenase & $1.00 \mathrm{E}-06$ & $-1.85^{*}$ \\
\hline 62 & Q61176 & Arg1 & Arginase-1 & $6.00 \mathrm{E}-07$ & $1.86^{*}$ \\
\hline 70 & Q78JT3 & Haao & 3-hydroxyanthranilate 3,4-dioxygenase & $1.70 \mathrm{E}-05$ & $-1.47^{*}$ \\
\hline 72 & Q99KR3 & Lactb2 & Beta-lactamase-like protein 2 & 0.00029 & $-1.86^{*}$ \\
\hline 78 & P19639 & Gstm3 & Glutathione S-transferase Mu 3 & 0.00063 & $-2.11^{*}$ \\
\hline 80 & 008709 & Prdx6 & Peroxiredoxin-6 & 0.0027 & $1.56^{*}$ \\
\hline 85 & P10648 & Gsta2 & Glutathione S-transferase A2 & 8.30E-05 & $1.54^{*}$ \\
\hline 86 & P10648 & Gsta2 & Glutathione S-transferase A2 & 0.013 & $1.66^{*}$ \\
\hline 89 & P35700 & $\operatorname{Prd} \times 1$ & Peroxiredoxin-1 & $1.60 \mathrm{E}-06$ & $1.18^{*}$ \\
\hline 91 & P10639 & Txn1 & Thioredoxin & 0.0011 & $1.39^{*}$ \\
\hline
\end{tabular}

${ }^{1}$ P-value from one way ANOVA statistical test between the experimental groups (paraquat, tetracycline, valproic acid, chlorpromazine, ethinylestradiol, negative controls D-mannitol and $\mathrm{LiCO}_{3}$ and the vehicle controls $\mathrm{DMSO}$ and PBS) with each five biological replicates.

${ }^{2}$ The difference in the standardized abundance of the proteins is expressed as the fold change between the control (C) and the treated groups $(\mathrm{T})$. The fold change is calculated by taking the means of standardized volume values for the protein spot in the corresponding groups (PBS = PBS vehicle control, $\mathrm{PQ}=$ paraquat), values are calculated as $\mathrm{T} / \mathrm{C}$ and displayed in the range of +1 to $+\infty$ for increases in expression and calculated as $-\mathrm{C} / \mathrm{T}$ and displayed in the range of $-\infty$ to -1 for decreased expression.

* Indicates significant fold changes $(\mathrm{P} \leq 0.05)$ between the control and the treated group, calculated with a multiple comparison test. 
Table 7.4: Protein identification of differentially expressed proteins in primary mouse hepatocytes after exposure to cholestasis inducers chlorpramazine and ethinylestradiol.

\begin{tabular}{|c|c|c|c|c|c|c|}
\hline \multirow[b]{2}{*}{ No } & \multirow{2}{*}{$\begin{array}{c}\text { Uniprot } \\
\text { Accession } \\
\text { No }\end{array}$} & \multirow[b]{2}{*}{$\begin{array}{l}\text { Gene } \\
\text { name }\end{array}$} & \multirow[b]{2}{*}{ Protein description } & \multirow{2}{*}{$\begin{array}{c}\text { P-value } \\
\text { (one-way } \\
\text { ANOVA) }\end{array}$} & \multicolumn{2}{|c|}{ Fold Change $^{2}$} \\
\hline & & & & & $\begin{array}{l}\text { DMSO/ } \\
\text { Chlor }\end{array}$ & $\begin{array}{c}\text { DMSO/ } \\
\text { EE }\end{array}$ \\
\hline 7 & P07901 & Hsp90aa1 & Heat shock protein HSP 90-alpha (HSP 86) & $4.90 \mathrm{E}-05$ & $1.09^{*}$ & $1.04^{*}$ \\
\hline 13 & P48678 & Lmna & Prelamin-A/C & 0.016 & 1.09 & -1.06 \\
\hline 24 & Q8VCU1 & Gm4738 & Liver carboxylesterase 3b & 0.0092 & 1.12 & $1.09 *$ \\
\hline 29 & Q8QZR3 & Ces2a & Carboxylesterase 6 & 0.011 & -1.01 & -1.04 \\
\hline 34 & Q00612 & G6pdx & Glucose-6-phosphate 1-dehydrogenase X & $3.90 \mathrm{E}-05$ & -1.07 & -1.24 \\
\hline 39 & P11679 & Krt8 & Keratin, type II cytoskeletal 8 & 0.0065 & $1.55^{*}$ & 1.21 \\
\hline 60 & P60710 & Actb & Actin, cytoplasmic 1 & 0.00066 & -1.6 & -1.82 \\
\hline 65 & P16858 & Gapdh & $\begin{array}{l}\text { Glyceraldehyde-3-phosphate dehydroge- } \\
\text { nase }\end{array}$ & 0.018 & 1.29 & 1.02 \\
\hline 77 & 008807 & Prdx4 & Peroxiredoxin-4 & 0.00046 & -1.22 & $-1.31^{*}$ \\
\hline
\end{tabular}

${ }^{1}$ P-value from one way ANOVA statistical test between the experimental groups (paraquat, tetracycline, valproic acid, chlorpromazine, ethinylestradiol, negative controls D-mannitol and $\mathrm{LiCO}_{3}$ and the vehicle controls $\mathrm{DMSO}$ and PBS) with each five biological replicates.

${ }^{2}$ The difference in the standardized abundance of the proteins is expressed as the fold change between the control (C) and the treated groups (T). The fold change is calculated by taking the means of standardized volume values for the protein spot in the corresponding groups (DMSO = DMSO vehicle control, Chlor = chlorpramazine, $\mathrm{EE}=\mathrm{ethi}-$ nylestradiol), values are calculated as T/C and displayed in the range of +1 to $+\infty$ for increases in expression and calculated as $-\mathrm{C} / \mathrm{T}$ and displayed in the range of $-\infty$ to -1 for decreased expression.

* Indicates significant fold changes $(\mathrm{P} \leq 0.05)$ between the control and the treated group, calculated with a multiple comparison test.

\section{Discriminant analysis of principal components}

The principle component analysis was performed based on the spot maps of the different compounds and also included the spot maps obtained from our previous study ${ }^{22}$. Table 7.1 shows an overview of the tested compounds with their drug class. The discriminant analysis of principal components showed that the cholestatic and necrotic compounds can be distinguished from all groups on principle component 2, which accounts for $24.7 \%$ of the total subject variance (Figure 7.3A). The compounds causing steatosis could be distinguished in the principle component 3 , which accounts for $21.6 \%$ of the total subject variance (Figure $7.3 \mathrm{~A}$ ). The most contributing spots are shown in Figure 7.3B. Unfortunately, we could not identify all the proteins from these spots. For necrosis, catalase (Cat), alanine aminotransferase 2 (Gpt2), UTP--glucose-1-phosphate uridylyltransferase (Ugp2), arginase-1 (Arg1), peroxiredoxin-6 (Prdx6) were identified as most contributing spots. For cholestasis, vitamin D-binding protein (Gc) and for steatosis carnitine O-palmitoyltransferase 2 (cpt2) were identified as most contributing spots. 


\section{DISCUSSION}

In this study the proteome of primary mouse hepatocytes was assessed for its ability to discriminate between different types of drug-induced hepatotoxicity. Primary mouse hepatocytes were exposed to six well-characterized hepatotoxicants: diclofenac and paraquat (necrosis), tetracycline and valproic acid (steatosis), chlorpramazine and ethinylestradiol (cholestasis), and two nephrotoxicants as negative controls: D-mannitol and $\mathrm{LiCO}_{3}$. Afterwards, changes in protein expression were determined by means of difference gel electrophoresis. With use of a discriminant principal component analysis we attempted to differentiate between the hepatotoxic phenotypes. In addition, the protein spot maps from a previous study were included which contained data from primary mouse hepatocytes exposed to acetaminophen, amiodarone and cyclosporin A, inducing necrosis, steatosis and cholestasis, respectively.

Previously, we investigated the cellular ${ }^{22}$ and secreted proteins from primary mouse hepatocytes treated with acetaminophen, amiodarone or cyclosporin A. Based on both the cellular and the secreted protein expression profiles cyclosporin A could be distinguished from control, acetaminophen- and amiodarone-treated samples. In addition, the secretome of primary mouse hepatocytes was also suitable for detecting acetaminophen-induced differentially expressed proteins. Here we used additional hepatotoxicants, to identify class-specific biomarkers.

Based on the cellular proteome ${ }^{22}$, it was shown that cyclosporin A induced endoplasmic reticulum (ER) stress and altered the ER-Golgi transport in primary mouse hepatocytes. Furthermore the differential expression of liver carboxylesterase and bile salt sulfotransferase was associated with a protective adaptive response against cyclosporin A-induced cholestasis. Here, ethinylestradiol and chlorpromazine were used as additional cholestatic compounds. Ethinylestradiol is a synthetic bioactive estrogen which is mainly used in formulations of combined oral contraceptives. Estrogens are known to cause cholestasis in susceptible women during pregnancy, after administration of oral contraceptives, or during postmenopausal replacement therapy ${ }^{26}$. To examine the mechanisms involved in estrogen-induced cholestasis, ethinylestradiol can be used as a model compound ${ }^{26,27}$.

Chlorpromazine is a neuroleptic drug which is used in the treatment of schizophrenia. However, during its therapeutical use side effects, including several cases of liver injury and particularly cholestasis, have been observed ${ }^{28,29}$. The exposure of primary mouse hepatocytes to the cholestatic compounds, induced only a mild effect on the proteome. While liver carboxylesterase 1 was down-regulated by cyclosporin $A$, ethinyl estradiol induced a small upregulation of liver carboxylesterase 3B. Carboxylesterases are involved in the detoxification of xenobiotics and in the activation of ester- and amide pro-drugs.

Out the PCA analysis, Gc was one of the most contributing protein spots responsible for the discrimination of the cholestatic compounds. Indeed, chlorpromazine induced an up- 
regulation of 1.47 and ethinylestradiol an up-regulation of 1.23 , however this up-regulation was not significant.

Tetracycline and valproic acid were used as model compounds for steatosis. Tetracycline is a broad-spectrum antibiotic ${ }^{30}$, and valproic acid is one of the most widely prescribed antiepileptic drugs worldwide. Despite their pharmacological importance and effectiveness, their potential hepatotoxicity is still a major concern. The cell culture medium of primary mouse hepatocytes treated with valproic acid and tetracycline showed both a $>2$-fold elevated concentration of TBIL. Increased bilirubin concentrations are usually associated with cholestasis, however together with AST it is the most important predictor of drug-induced hepatotoxicity ${ }^{25}$. In contrast to valproic acid, tetracycline induced a minor effect on the proteome of primary mouse hepatocytes.

Valproic acid is an eight-carbon branched-chain fatty acid and is mainly metabolized in mitochondria by means of the fatty acid $\beta$-oxidation, and a minor in the endoplasmic reticulum, yielding $\Delta^{4}$-valproic acid. In order to enter the $\beta$-oxidation cycle, both valproic acid and $\Delta^{4}$-valproic acid need to be activated to valproyl-CoA and $\Delta^{4}$-valproyl-CoA, respectively ${ }^{31}$. This reaction is catalyzed by ATP-dependent acyl-coA ligases, located on the outer side of the mitochondrial membrane ${ }^{32}$. However, the acyl-coA ligase(s) responsible for the activation of valproic acid have not been identified yet ${ }^{33}$. Acyl-CoA synthetase family member 2 (Acsf2) and long-chain-fatty-acid-CoA ligase 1 (Acs/1) are probably good candidates. Here valproic acid induced a down-regulation of Acsf2 and Acsl1, which can cause an accumulation of fatty acids in the cytosol. After the activation both valproyl-CoA and $\Delta^{4}$-valproyl-CoA can enter the mitochondria via the carnitine shuttle, which involves carnitine palmitoyltransferase I (Cpt1), carnitine-acylcarnitine translocase (Cact) and carnitine palmitoyltransferase II (Cpt2) ${ }^{33}$. Previously, it was shown that both valproyl-CoA and $\Delta^{4}$-valproyl-CoA inhibit the activity of Cpt1 ${ }^{34}$. Cpt1 is the rate-limiting enzyme mediating the transport of valproyl-CoA and $\Delta^{4}$-valproyl-CoA to the inside of the mitochondria. Inhibition of Cpt1 automatically affects Cpt2, which was significantly up-regulated in our study. Valproyl-CoA or $\Delta 4$-valproyl-CoA thioester generated by $\mathrm{Cpt} 2$ will be dehydrogenated in the $\beta$-oxidation cycle. In addition, in the PCA analysis Cpt2 was one of the most contributing protein spots responsible for the discrimination of the steatosic compounds.

One of the main enzymes responsible for the dehydrogenation of long-chain fatty acids is very long-chain specific acyl-CoA dehydrogenase (Acadvl) ${ }^{35,36}$. Here, valproic acid induces an increased expression of Acadvl. Both, the increased expression of Cpt2 and Acadvl can be seen as a protective adaptive response against valproic acid-induced hepatotoxicity.

Mitochondrial dysfunction is central in the pathophysiology of steatosis. Besides the differential expression of several proteins from the $\beta$-oxidation other mitochondrial proteins like mitochondrial inner membrane protein and voltage-dependent anion-selective channel protein1 (Vdac1) were differentially expressed by valproic acid. Vdac1 is a member of the mitochondrial permeability transition pores (MPTP). Several drugs, or high levels of endogenous 
derivatives like calcium, fatty acids, and bile salts can induce MPTP opening ${ }^{37}$, which strongly alters mitochondrial function and structure ${ }^{37}$.
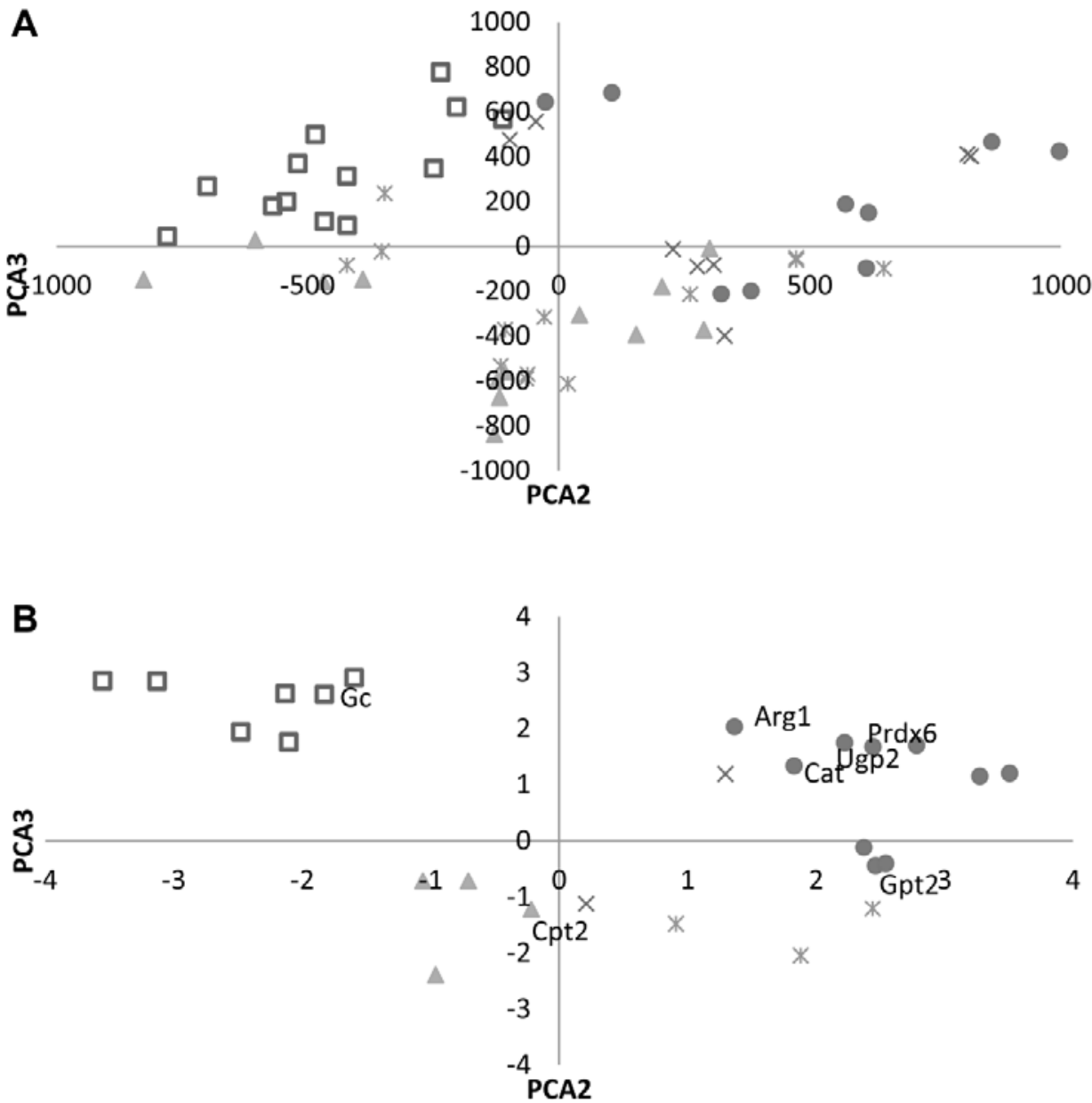

- Necrosis $\square$ Cholestasis $\triangle$ Steatosis $\times$ Negative Control $*$ Control

Figure 7.3: discriminant PCA of the spot intensities based on the drug classes cholestasis, steatosis and necrosis. (A) Score plot. (B) Loading plot. The top $10 \%$ of variables with a loading score $>2.7$ are indicated.

To study drug-induced necrosis, paraquat was used as a model compound. Paraquat is a widely used herbicide ${ }^{38}$. In case of accidental poisoning paraquat will induce liver necrosis, mediated by the production of reactive oxygen species (ROS) such as hydrogen peroxide and hydroxyl radicals, leading to oxidative stress. Anti-oxidant enzymes play an important role in the protection against oxidative stress. Catalase, one of the most important enzymes of the cellular anti-oxidant defense system was up-regulated after paraquat exposure. In addition, para- 
quat also induced an increased expression of the anti-oxidant enzymes peroxiredoxin 1 and 6 and thioredoxin. Peroxiredoxins use thioredoxin to detoxify hydrogen peroxide. Furthermore, The anti-oxidants Cat and Prdx6 were two of the most contributing protein spots responsible for the discrimination of the necrotic compounds. ThiseEmphasizes the value of anti-oxidant enzymes as candidate biomarkers for identification of necrotic hepatotoxicants.

In addition, paraquat also induced an increased expression of glutathione-S- transferases A2 and Mu3. Glutathione-S-transferases conjugate reduced gluthatione to electrophilic centers of several toxicants in order to make them more soluble.

Remarkably, several metal-binding proteins like Aco1 and 2, Hgd, Haao, sord and lactb2 were down-regulated after paraquat exposure. Possibly, these metal-binding proteins are vulnerable targets for oxygen radicals. Aco 1 and 2, Hgd and Haao bind to iron, while sord and lactb2 are zinc-containing enzymes. Iron and other transition metals are known to enhance the conversion of superoxide and hydrogen peroxide into the highly toxic free hydroxyl radicals ${ }^{39}$ Previously it was shown that paraquat induces oxidation of the $[4 \mathrm{Fe}-4 \mathrm{~S}]^{2+}$ cluster in Aco1 and $2^{40}$. This oxidation destabilizes the complex and promotes release of the labile iron atom. Consequently, hydrogen peroxide is formed by the reduction of superoxide ${ }^{40,41}$. Although, iron usually enhances the formation of free radicals, other trace elements, such as zinc and selenium, protect against the harmful effects of these radicals ${ }^{42}$. Zinc has the capacity to antagonize transition metal-mediated oxidations and transfer of electrons under normal and pathologic conditions ${ }^{43}$. Probably, the down-regulation of these metal-binding proteins is an adaptive response to the intracellular redox status and the formation of free radicals.

The primary mouse hepatocytes treated with the nephrotoxic compounds D-mannitol or $\mathrm{LiCO}_{3}$ did not show an increase of serum aminotransferases and LD in cell culture medium. In addition we could not identify any differentially expressed proteins on the proteome map from the primary mouse hepatocytes treated with D-mannitol or $\mathrm{LiCO}_{3}$. This indicates, that primary mouse hepatocytes specifically responds to hepatotoxicants and will not respond to other organ toxic compounds.

The objective of this study was to investigate whether compounds inducing similar toxicological endpoints produce similar changes in protein expression. By means of a discriminant principal component analysis a moderate discrimination of the hepatotoxic phenotypes was achieved. In this analysis the necrotizing compounds were mainly discriminated due to the expression of Cat, Gpt2, Ugp2, Arg1 and Prdx6. Compounds which induce cholestasis can be distinguished due to the expression of Gc and compounds which induce steatosis can be distinguished by means of the expression of Cpt2. Whether these proteins can be used as classspecific biomarkers needs further validation. 


\section{CONCLUSION}

Based on a PCA analysis of the spot intensities from 2-DE proteome maps, the proteome of the primary mouse hepatocytes was able to distinguish the necrotic and cholestatic inducers,. The steatosis-inducers were more difficult to distinguish. Potential distinguishing marker proteins were proposed. In addition, several hepatotoxic mechanisms related to the different phenotypes were identified. Furthermore, the nephrotoxic compounds which served as negative controls did not induce hepatoxic effects, indicating the specific response of primary mouse hepatocytes to hepatotoxicants.

Nevertheless, the classification of drug-induced hepatotoxicity remains complicated, since some phenotypical characteristics can overlap as for example elevated TBIL levels seen with the steatosis-inducers tetracycline and valproic acid, while this is more typical for cholestatic compounds.

\section{ACKNOWLEDGMENTS}

We thank Erik Royackers from the Biomedical Research Institute of Hasselt University for his technical support of the LC MS/MS analysis. We also thank dr. Will Wodzig and his group from Maastricht UMC+ for the analysis of the clinical parameters. 


\section{REFERENCES}

1 Johnson, D. E. \& Wolfgang, G. H. Predicting human safety: screening and computational approaches. Drug discovery today 5, 445-454 (2000).

2 Olson, H., Betton, G., Stritar, J. \& Robinson, D. The predictivity of the toxicity of pharmaceuticals in humans from animal data--an interim assessment. Toxicol Lett 102-103, 535-538 (1998).

3 Suter, L. et al. EU Framework 6 Project: Predictive Toxicology (PredTox)-overview and outcome. Toxicol Appl Pharmacol 252, 73-84 (2010).

4 van Kesteren, P. C. et al. Deregulation of cancer-related pathways in primary hepatocytes derived from DNA repair-deficient Xpa-/-p53+/- mice upon exposure to benzo[a]pyrene. Toxicol Sci 123, 123-132 (2011).

5 Mathijs, K. et al. Assessing the metabolic competence of sandwich-cultured mouse primary hepatocytes. Drug Metab Dispos 37, 1305-1311 (2009).

6 Nelson, S. D. Mechanisms of the formation and disposition of reactive metabolites that can cause acute liver injury. Drug Metab Rev 27, 147-177 (1995).

7 Pauli-Magnus, C. \& Meier, P. J. Hepatobiliary transporters and drug-induced cholestasis. Hepatology 44, 778-787 (2006).

8 Fromenty, B. et al. Amiodarone inhibits the mitochondrial beta-oxidation of fatty acids and produces microvesicular steatosis of the liver in mice. J Pharmacol Exp Ther 255, 1371-1376 (1990).

9 Kienhuis, A. S. et al. Application of toxicogenomics in hepatic systems toxicology for risk assessment: acetaminophen as a case study. Toxicol Appl Pharmacol 250, 96-107 (2011).

10 Waring, J. F., Ciurlionis, R., Jolly, R. A., Heindel, M. \& Ulrich, R. G. Microarray analysis of hepatotoxins in vitro reveals a correlation between gene expression profiles and mechanisms of toxicity. Toxicology letters 120, 359368 (2001).

11 de Longueville, F. et al. Use of a low-density microarray for studying gene expression patterns induced by hepatotoxicants on primary cultures of rat hepatocytes. Toxicological sciences : an official journal of the Society of Toxicology 75, 378-392 (2003).

12 Mathijs, K. et al. Discrimination for genotoxic and nongenotoxic carcinogens by gene expression profiling in primary mouse hepatocytes improves with exposure time. Toxicol Sci 112, 374-384 (2009).

13 Bartosiewicz, M. J., Jenkins, D., Penn, S., Emery, J. \& Buckpitt, A. Unique gene expression patterns in liver and kidney associated with exposure to chemical toxicants. The Journal of pharmacology and experimental therapeutics 297, 895-905 (2001).

14 Aardema, M. J. \& MacGregor, J. T. Toxicology and genetic toxicology in the new era of 'toxicogenomics': impact of '-omics' technologies. Mutat Res 499, 13-25 (2002).

15 Yamamoto, T., Kikkawa, R., Yamada, H. \& Horii, I. Identification of oxidative stress-related proteins for predictive screening of hepatotoxicity using a proteomic approach. J Toxicol Sci 30, 213-227 (2005).

16 Yamamoto, T., Kikkawa, R., Yamada, H. \& Horii, I. Investigation of proteomic biomarkers in in vivo hepatotoxicity study of rat liver: toxicity differentiation in hepatotoxicants. J Toxicol Sci 31, 49-60 (2006).

17 Thome-Kromer, B. et al. Toward the identification of liver toxicity markers: a proteome study in human cell culture and rats. Proteomics 3, 1835-1862 (2003).

18 Van Summeren, A. et al. Proteomics Investigations of Drug-Induced Hepatotoxicity in HepG2 Cells. Toxicol Sci 120, 109-122 (2011).

19 Meneses-Lorente, G. et al. Identification of early proteomic markers for hepatic steatosis. Chem Res Toxicol 19, 986-998 (2006).

20 Seglen, P. O. Preparation of isolated rat liver cells. Methods Cell Biol 13, 29-83 (1976).

21 Mosmann, T. Rapid colorimetric assay for cellular growth and survival: application to proliferation and cytotoxicity assays. J Immunol Methods 65, 55-63 (1983).

22 Van Summeren, A. et al. Screening for drug-induced hepatotoxicity in primary mouse hepatocytes using acetaminophen, amiodarone, and cyclosporin a as model compounds: an omics-guided approach. Omics : a journal of integrative biology 17, 71-83 (2013). 
23 Bouwman, F. G. et al. The Physiologic Effects of Caloric Restriction Are Reflected in the in Vivo AdipocyteEnriched Proteome of Overweight/Obese Subjects. J Proteome Res 8, 5532-5540 (2009).

24 Dumont, D., Noben, J. P., Raus, J., Stinissen, P. \& Robben, J. Proteomic analysis of cerebrospinal fluid from multiple sclerosis patients. Proteomics 4, 2117-2124 (2004).

25 Bjornsson, E. \& Olsson, R. Outcome and prognostic markers in severe drug-induced liver disease. Hepatology 42, 481-489 (2005).

26 Yamamoto, Y. et al. Estrogen receptor alpha mediates 17alpha-ethynylestradiol causing hepatotoxicity. The Journal of biological chemistry 281, 16625-16631 (2006).

27 Lee, J. M. et al. Expression of the bile salt export pump is maintained after chronic cholestasis in the rat. Gastroenterology 118, 163-172 (2000).

28 Regal, R. E., Billi, J. E. \& Glazer, H. M. Phenothiazine-induced cholestatic jaundice. Clin Pharm 6, 787-794 (1987).

29 Antherieu, S. et al. Oxidative stress plays a major role in chlorpromazine-induced cholestasis in human heparg cells. Hepatology 57, 1518-1529 (2013).

30 Yin, H. Q. et al. Hepatic gene expression profiling and lipid homeostasis in mice exposed to steatogenic drug, tetracycline. Toxicological sciences : an official journal of the Society of Toxicology 94, 206-216 (2006).

31 Aires, C. C. et al. Studies on the extra-mitochondrial CoA -ester formation of valproic and Delta4 -valproic acids. Biochimica et biophysica acta 1771, 533-543 (2007).

32 Ponchaut, S., van Hoof, F. \& Veitch, K. In vitro effects of valproate and valproate metabolites on mitochondrial oxidations. Relevance of CoA sequestration to the observed inhibitions. Biochemical pharmacology 43, 24352442 (1992).

33 Silva, M. F. et al. Valproic acid metabolism and its effects on mitochondrial fatty acid oxidation: a review. Journal of inherited metabolic disease 31, 205-216 (2008).

34 Aires, C. C. et al. Inhibition of hepatic carnitine palmitoyl-transferase I (CPT IA) by valproyl-CoA as a possible mechanism of valproate-induced steatosis. Biochemical pharmacology 79, 792-799 (2010).

35 Fromenty, B. \& Pessayre, D. Inhibition of mitochondrial beta-oxidation as a mechanism of hepatotoxicity. Pharmacol Ther 67, 101-154 (1995).

36 Izai, K., Uchida, Y., Orii, T., Yamamoto, S. \& Hashimoto, T. Novel fatty acid beta-oxidation enzymes in rat liver mitochondria. I. Purification and properties of very-long-chain acyl-coenzyme A dehydrogenase. The Journal of biological chemistry 267, 1027-1033 (1992).

37 Begriche, K., Massart, J., Robin, M. A., Borgne-Sanchez, A. \& Fromenty, B. Drug-induced toxicity on mitochondria and lipid metabolism: mechanistic diversity and deleterious consequences for the liver. Journal of hepatology $\mathbf{5 4}$ 773-794 (2011).

38 Haley, T. J. Review of the toxicology of paraquat (1,1'-dimethyl-4,4'-bipyridinium chloride). Clin Toxicol 14, 1-46 (1979).

39 Hershko, C. \& Weatherall, D. J. Iron-chelating therapy. Crit Rev Clin Lab Sci 26, 303-345, (1988).

40 Cantu, D., Fulton, R. E., Drechsel, D. A. \& Patel, M. Mitochondrial aconitase knockdown attenuates paraquatinduced dopaminergic cell death via decreased cellular metabolism and release of iron and $\mathrm{H}(2) \mathrm{O}(2)$. J Neurochem 118, 79-92 (2011).

41 Flint, D. H., Tuminello, J. F. \& Emptage, M. H. The inactivation of Fe-S cluster containing hydro-lyases by superoxide. The Journal of biological chemistry 268, 22369-22376 (1993).

42 Coudray, C., Rachidi, S. \& Favier, A. Effect of zinc on superoxide-dependent hydroxyl radical production in vitro. Biol Trace Elem Res 38, 273-287 (1993).

43 Powell, S. R. The antioxidant properties of zinc. J Nutr 130, 1447S-1454S (2000). 


\section{CHAPTER 8}

Summary and general discussion 
The safety assessment of potential drug candidates remains a challenge for the pharmaceutical industry. Consequently, unexpected hepatotoxicity is one of the major reasons why candidate-drugs fail during preclinical or clinical trials. The liver is responsible for the metabolism of drugs and toxic compounds, thus it is vulnerable for drug-induced toxicity. This emphasizes the need for new screening methods that address toxicological hazards early in the drug discovery process. These new screening methods should improve the prediction, characterization and understanding of drug-induced hepatotoxicity. Consequently, this will lead to safer drugs and a more efficient drug discovery and development process.

The use of genomics-based technologies in toxicology is called 'toxicogenomics'. The application of genomics-based technologies, enable to investigate the function of the complete genome by expression analyses at the level of transcription (transcriptomics), translation (proteomics) and metabolism (metabonomics). Through simultaneous measurements of global gene expression, proteins or metabolites it is possible to identify specific hepatotoxic pathways and mechanisms. In addition, by means of toxicogenomics it is possible to select similar endpoints in vivo and in vitro, enabling to perform in vitro to in vivo comparisons. This allows the development and use of in vitro models for the prediction of hepatotoxic responses in humans. Also, it is preferred to correctly classify hepatotoxicants based on toxicity pathways related to their hepatotoxic phenotype ${ }^{1}$. Hepatotoxic phenotypes observed upon chemical exposure often fall into one of the following categories: 1 ) necrosis, a form of premature cell death due to external factors like free radicals and/or toxic metabolites; 2 ) hepatic steatosis, thought to occur due to mitochondrial damage causing impairment of $\beta$-oxidation and accumulation of small lipid vesicles within hepatocytes; 3 ) cholestasis, which represents failure of bile excretion, often caused by inhibition of bile salt transporters leading to the intracellular accumulation of bile.

The aim of this thesis was to obtain insight in the use of in vitro cell systems for classification of hepatotoxic compounds, and in the hepatotoxic effects of those compounds at the molecular and pathway level. To that purpose, a toxicogenomics approach was used, with the focus on proteomics, enriched with transcriptomics data.

\section{TOXICOLOGICAL RESPONSES IN PRIMARY MOUSE HEPATOCYTES}

Currently, hepatic cell lines, primary hepatocytes or precision cut liver slices from several species are the most common in vitro models used for hepatotoxicity screening. The use of primary hepatocytes is preferred because of their liver specific characteristics, in particular their drug metabolizing capacity. While primary rat and human hepatocytes are well described in vitro systems, studies using primary mouse hepatocytes are limited. However, it was shown that primary mouse hepatocytes maintain their metabolic competence better compared to primary rat hepatocytes ${ }^{2}$. In addition, transgenic mouse models, provide primary hepatocytes suitable for dedicated mechanistic investigations of liver toxicity ${ }^{3}$. In chapter 3 we analyzed the cellular proteome of primary mouse hepatocytes for its ability to detect 
drug-induced hepatotoxicity. For this study, three well-defined hepatotoxicants (acetaminophen, amiodarone and cyclosporin A) were selected. Each compound induces in vivo a different phenotype of hepatotoxicity: acetaminophen for necrosis, amiodarone for steatosis and cyclosporin A for cholestasis. After exposure to these hepatotoxic compounds, the proteome of primary mouse hepatocytes was analyzed. It appeared possible to distinguish the protein expression profile of cyclosporin A from the control, as well as from acetaminophen and amiodarone-treated samples. However, the proteome of primary mouse hepatocytes was not able to make an appropriate differentiation between acetaminophen, amiodarone and the control.

The cellular proteome of primary mouse hepatocytes showed that cyclosporin A induced molecular mechanisms related to endoplasmic reticulum (ER) stress and to an altered ERGolgi transport. ER stress can be caused by accumulated cholesterol in the ER ${ }^{4}$. Production and secretion of bile acids is an important route for the elimination of excessive cholesterol ${ }^{5}$. Inhibition of bile salt transport proteins by cyclosporin A, will not only lead to the accumulation of bile acids but it will also increase the hepatic pool of free cholesterol. Furthermore, the cyclosporin A-induced proteome revealed a modulation of mechanisms to remove the excess of intracellular cholesterol. These mechanisms, involve a down-regulation of Ces1 and the differential expression of several phase II detoxifying enzymes.

ER stress and an altered the ER-Golgi transport caused by the intracellular accumulation of cholesterol can result in an inhibited protein secretion. This led us to analyze the proteins secreted by these primary mouse hepatocytes after administration of acetaminophen, amiodarone and cyclosporin A, as described in chapter 4.

The proteins secreted by hepatocytes contain a conceivable amount of candidate biomarkers for drug-induced hepatotoxicity. This is because, besides the detoxification of toxic compounds, the liver is also responsible for the production and secretion of a large variety of serum proteins. Consequently, when specific protein markers for hepatotoxicity can be found in the secretome of hepatocytes, it is likely that these markers are present in serum and available for diagnosing hepatotoxicity in patients or subjects from clinical trials. However, the analysis of proteins secreted by cultured cells is challenged by the presence of cytoplasmic proteins, released in the cell culture medium after cell death. For that reason we have used a secretion blocking strategy by means of a reduction of the incubation temperature to $20^{\circ} \mathrm{C}$, to verify the active secretion of the proteins.

Analysis of the baseline secretome of primary mouse hepatocytes showed that these cells secrete a considerable amount of plasma proteins such as albumin, ceruloplasmin and antithrombin-III. This indicates that liver-specific characteristics regarding protein secretion in primary mouse hepatocytes are well preserved.

Based on the findings from chapter 3, we hypothesized that the ER stress induced by cyclosporin A may result in an inhibited protein secretion. Indeed, the secretome of cyclosporin A treated primary mouse hepatocytes showed indeed a decreased secretion of particular proteins. However, cyclosporin A also increased the secretion of several plasma proteins like 
ApoA1, ApoA4, ApoE, haptoglobulin, hemopexin and serum albumin. Furthermore, some of these plasma proteins were also up-regulated after exposure to acetaminophen or amiodarone. These findings were unexpected, since liver failure is mostly accompanied with a decreased plasma level of serum albumin. Serum albumin in known to scavenge toxicological compounds, therefore an increased expression of serum albumin is probably a protective response in an early stage of toxicity. When liver failure occurs in a later stage of toxicity, the liver will no longer be able to produce adequate amounts of albumin, which will result in decreased levels of serum albumin.

Besides the differential expression of generic proteins which may be released into plasma, in primary mouse hepatocytes, the hepatotoxicants induced the secretion of proteins related to an impaired cholesterol and lipid metabolism. While one would expect these proteins to be differentially expressed by steatosis- and/or cholestasis-inducing compounds, some of these proteins also showed up after an acetaminophen treatment. These findings indicate an overlap between some molecular mechanisms inducing phenotypically characteristics of the model-hepatotoxicants. On the other hand, the differential expression of vitamin-D-binding protein (Gc) and farnesyl pyrophosphate synthetase (Fdps), two proteins related to cholesterol metabolism, were only induced by cyclosporin A.

This thesis mainly focuses on the use of acetaminophen, amiodarone and cyclosporin $A$ as prototypical compounds for hepatotoxicity. Besides the detection of hepatotoxicity per se it is preferred to correctly classify hepatotoxicants according to their hepatotoxic phenotype. Compounds which induce similar toxicological endpoints in the liver, might produce similar changes in protein expression. In order to obtain further insight in the use of primary mouse hepatocytes for the classification of hepatotoxic compounds, it was necessary to extend the number of analyzed compounds per category. In chapter 7 we describe the protein expression of primary mouse hepatocytes exposed to six well-characterized hepatotoxicants selected based on their different hepatotoxic phenotypes: diclofenac and paraquat (necrosis), tetracycline and valproic acid (steatosis), chlorpramazine and ethinylestradiol (cholestasis), and two nephrotoxicants as negative controls: D-mannitol and $\mathrm{LiCO}_{3}$. Afterwards, the protein spot maps from chapter 7 were combined with the spot maps from chapter 3, where primary mouse hepatocytes were exposed to acetaminophen, amiodarone and cyclosporin $A$, inducing necrosis, steatosis and cholestasis, respectively. A discriminant principal component analysis was used to differentiate between the different hepatotoxic phenotypes. The necrotizing compounds were mainly discriminated on the basis of the differential expression of catalase, glutamic pyruvate transaminase 2, UDP-glucose pyrophosphorylase 2, arginase 1 and peroxiredoxin 6. Compounds which induce cholestasis could be distinguished on the basis of the expression of Gc and compounds which induce steatosis, were distinguished by means of Cpt2. Further validation will indicate whether these proteins can be used as class-specific biomarkers. 


\section{MOUSE-HUMAN INTERSPECIES COMPARISONS}

To predict if an adverse response determined in primary mouse hepatocytes, is conserved in humans, it is necessary to extrapolate the adverse responses from a mouse to a human model. Primary human hepatocytes are most suitable for a direct mouse-human interspecies comparison. However, their use is complicated by the considerable inter-individual variability caused by genetic, life style and age differences of the donors. In addition, suitable liver samples as a source of primary human hepatocytes are only limitedly available. To overcome these restrictions, we chose to work with HepG2 cells, a human hepatoma cell line, which can be cultured in large amounts. In contrast to primary hepatocytes, HepG2 cells are sufficiently available. They are easier to culture, have a longer life span, demonstrate less biological variation and they are widely used in toxicological and toxicogenomics studies ${ }^{6,7}$. Despite the advantages of HepG2, it is fair to note that they have a lower expression of certain phase I drug metabolizing enzymes ${ }^{8,9}$. In chapter 2 we analyzed the cellular proteome of HepG2 cells after exposure to acetaminophen, amiodarone and cyclosporin A. The results from this study permit a direct comparison with the protein expression of primary mouse hepatocytes exposed to the same compounds, as described in chapter 3.

Both in vitro systems affected similar pathways after exposure to cyclosporin A. Cyclosporin A induced in both models ER stress and affected the ER-Golgi transport, probably a result from intracellular cholesterol accumulation. In addition, both in vitro models show the differential expression of mitochondrial proteins, proteins involved in ATP-binding and carbohydrate metabolism. These results indicate that the protein expression induced by the hepatotoxicants and cyclosporin A in particular, is conserved between primary mouse hepatocytes and human HepG2 cells.

These conserved responses are also represented in the gene expression profiles as described in Chapter 5. In contrast to the current protemics techniques, a transcriptomics approach using micro-array data enables the analysis of the whole genome gene expression. These data allows a comprehensive pathway analysis of toxicological mechanisms. This study, revealed that corresponding hepatotoxicants induced similar patterns of gene expression in both hepatic in vitro models. Particularly, mechanisms related to cholesterol metabolism. In addition, the pathway analysis of the transcriptomic data reveals hepatotoxicity-associated conserved responses among HepG2 and primary mouse hepatocytes. These conserved responses indicate that adverse reactions in primary hepatocytes have the capacity to predict hepatotoxicty in humans.

Besides the similarities between HepG2 and primary mouse hepatocytes, HepG2 cells showed the differential expression of proteins identifying the carcinoma character of the HepG2 cell line. These included proteins responsible for mRNA processing such as heterogeneous nuclear ribonucleoproteins (hnRNP). hnRNPs play a role in tumor development and are up-regulated in various cancers ${ }^{10}$. 


\section{IN VITRO TO IN VIVO COMPARISONS}

In Chapter 6 of this thesis, in vitro to in vivo comparisons of the protein expression profiles were performed using the model compound cyclosporin A.

In vitro as well as in vivo, it was shown that cyclosporin A induced the differential expression of proteins related to cholesterol metabolism, a known side-effect of cyclosporin A. As mentioned before, accumulation of intracellular cholesterol can lead to ER stress ${ }^{4}$. While in vitro evidence was found for ER stress by the expression of several chaperone proteins, this effect was less obvious in vivo.

In vivo the main target of cyclosporin A are the mitochondria instead of the ER. However, HepG2 as well as the primary mouse hepatocytes also show the differential expression of several mitochondrial proteins after exposure to cyclosporin A, albeit to a lesser extent than in vivo.

These differences indicates that in vitro cell cultures can respond differently compared to the in vivo situation, probably because they are in direct contact with the toxicant and they function independently from other cells/organs. These differences between in vitro and vivo models explain the current difficulties to validate in vitro biomarkers towards in vivo models. Nevertheless, despite the toxicological response in vitro is slightly different from the in vivo situation, it was shown that the current in vitro models have the potential to distinguish different types of drug-induced hepatotoxicity.

\section{LIMITATIONS AND FURTHER RECOMMENDATIONS}

Not all the hepatotoxic compounds analyzed in this thesis showed a genuine toxicological response on the proteome of the used in vitro models. Probably this is due to a decreased expression of certain drug metabolizing enzymes in the in vitro models. In addition, the classification of drug-induced hepatotoxicity is complicated by the overlap of some phenotypical characteristics of different classes of hepatotoxicity.

To obtain a broader insight in the hepatotoxic mechanisms it is recommended to compare data from transcriptomics, proteomics and metabolomics experiments in a systems biology approach. In this thesis, the data obtained from proteome analysis was partially supplemented with complementary transcriptome data. Despite the fact that several bioinformatics tools are available, direct comparison of proteomic and transcriptomic expression data from divergent platforms remains challenging. By means of microarrays it is possible to analyze an almost complete set of target transcripts, whereas this is not the case by using standard proteomics techniques. Proteome analysis by means of DIGE is biased towards the detection of soluble proteins with non-extreme pl values. These limitations can be partly overcome by combining a gel-based approach with shotgun proteomics, linking high performance liquid chromatography with mass spectrometry. However, even after combining both techniques, 
the coverage of differentially expressed proteins which can be detected with proteomics technologies is less than the number of gene alterations covered by microarray procedures. For that reason, a direct comparison of proteomics and transcriptomics data is inherently biased towards highly expressed genes. Ultimately, both technologies studying gene or protein expression contribute to a systems-level understanding of drug-induced hepatotoxicity. In the immanent future, the development of new analytical software will further enhance the power of the emerging high-throughput technologies in proteomics as well as in transcriptomics.

The in vitro models used in this thesis have some limitations, which hampers the extrapolation of in vitro hepatotoxic effects to human in vivo effects.

In general single-cell in vitro models are limited with respect to extrapolation of biological effects to the in vivo situation. The in vitro models used in this thesis rely only on the use of hepatocytes, whereas other liver cells like Kupffer and stellate cells are absent. These cells often play an important role in the mediation of hepatotoxicity ${ }^{11}$. Hepatotoxic compounds can activate Kupffer cells, which secrete pro-inflammatory cytokines and superoxides. These cytokines can influence the expression of biotransformation enzymes and affect the stellate cells. Activated stellate cells show an enhanced collagen synthesis, which eventually will result in fibrosis. In contrast to hepatocyte-based in vitro models, precision cut liver slices preserves the three-dimensional structure of the liver. In addition they contain all the cell types which are present in the liver in vivo.

Furthermore, translation of effects seen in a hepatocyte-based in vitro model to the in vivo situation is also hampered by the absence of blood flow. A continuous blood flow enables exchanges of proteins and metabolites between hepatocytes and other cells and organs. Altogether, future efforts will continue to search for the optimal in vitro model system. For instance, the proteome of HepG2 cells indicates that the HepG2 cells can be used to analyze the cholestatic properties of cyclosporin A. However, their applicability for toxicity screening is often questioned because they have a lower expression level of some drug metabolizing enzymes ${ }^{8,9}$. The development of new cell lines such as HepaRG cells, which better retain their drug metabolism capacity, hold the promise to deliver a convenient in vitro model with the characteristics of primary hepatocytes.

Despite the conserved toxicological responses in the primary mouse hepatocytes, mice are not men. The use of hepatocytes from genetically identical mouse facilitates the study of hepatotoxic responses, while human hepatocytes have major inter-individual variability. While inter-individual differences are mostly a disadvantage in in vitro toxicology, the interindividual effects observed in primary human hepatocytes reflects the genuine phenotypic variability in the human population. A panel of primary hepatocytes composed from various genetically different donors might overcome these limitations. 


\section{CONCLUSION}

The research described in this thesis showed the relevance of hepatocyte-based in vitro models for classifying well-known hepatotoxicants based on the molecular toxicological effects of those compounds. The presented studies showed the value of proteomics data of cellular and secreted proteins for the identification of toxicological mechanisms.

Comparison of the gene and protein expression profiles of HepG2 with those of the primary mouse hepatocytes showed on both levels a strong conservation of the toxicological responses. The in vitro to in vivo comparisons of the cyclosporin A-induced protein expression profiles, revealed similar responses regarding ATP-binding, cholesterol and carbohydrate metabolism. Despite these similar responses induced by cyclosporin A, the in vivo data showed that the mitochondrial proteins were mainly attacked whereas in vitro the ER was mainly affected. These different toxicant-induced responses between in vivo and in vitro models can complicate the validation of in vitro biomarkers towards in vivo models. Nevertheless, it was shown that the current in vitro models have the potential to distinguish different types of drug-induced hepatotoxicity and can be used for diagnostic purposes.

This research has laid a foundation for the further development of hepatocytes-based in vitro assays to identify and classify possible hepatoxicants by means of an 'omics approach. 


\section{REFERENCES}

1 Kienhuis, A. S. et al. Application of toxicogenomics in hepatic systems toxicology for risk assessment: acetaminophen as a case study. Toxicol Appl Pharmacol 250, 96-107 (2011).

2 Mathijs, K. et al. Assessing the metabolic competence of sandwich-cultured mouse primary hepatocytes. Drug Metab Dispos 37, 1305-1311 (2009).

3 van Kesteren, P. C. et al. Deregulation of cancer-related pathways in primary hepatocytes derived from DNA repair-deficient Xpa-/-p53+/- mice upon exposure to benzo[a]pyrene. Toxicol Sci 123, 123-132 (2011).

4 Kockx, M. et al. Cholesterol accumulation inhibits ER to Golgi transport and protein secretion: studies of apolipoprotein E and VSVGt. The Biochemical journal 447, 51-60 (2012).

5 Zhao, B., Natarajan, R. \& Ghosh, S. Human liver cholesteryl ester hydrolase: cloning, molecular characterization, and role in cellular cholesterol homeostasis. Physiol Genomics 23, 304-310 (2005).

6 Van Summeren, A., Renes, J., Mariman, E. C., Kleinjans, J. C. \& van Delft, J. H. Response to Pathophysiological Relevance of Proteomics Investigations of Drug-Induced Hepatotoxicity in HepG2 Cells. Toxicol Sci 121, 431-433 (2011)

7 Knasmuller, S. et al. Use of human-derived liver cell lines for the detection of environmental and dietary genotoxicants; current state of knowledge. Toxicology 198, 315-328 (2004).

8 Boess, F. et al. Gene expression in two hepatic cell lines, cultured primary hepatocytes, and liver slices compared to the in vivo liver gene expression in rats: possible implications for toxicogenomics use of in vitro systems. Toxicol Sci 73, 386-402 (2003).

9 Wilkening, S. \& Bader, A. Influence of culture time on the expression of drug-metabolizing enzymes in primary human hepatocytes and hepatoma cell line HepG2. J Biochem Mol Toxicol 17, 207-213 (2003).

10 Carpenter, B. et al. The roles of heterogeneous nuclear ribonucleoproteins in tumour development and progression. Biochim Biophys Acta 1765, 85-100 (2006).

11 Guyot, C. et al. Hepatic fibrosis and cirrhosis: the (myo)fibroblastic cell subpopulations involved. Int J Biochem Cell Biol 38, 135-151 (2006). 
Nederlandse samenvatting 
Een correcte risicoanalyse van nieuwe geneesmiddelen is nog steeds een uitdaging voor de farmaceutische industrie. Het onverwacht optreden van leverbeschadiging tijdens het ontwikkelingsproces is één van de meest voorkomende redenen waarom sommige kandidaat geneesmiddelen niet op de markt gebracht kunnen worden. De lever is vatbaar voor beschadiging omdat het verantwoordelijk is voor de omzetting en verwijdering van giftige stoffen. Vaak wordt deze levertoxiciteit pas opgemerkt in een latere fase van de ontwikkeling van een nieuw geneesmiddel of zelfs pas tijdens klinische studies. Er is daarom nood aan nieuwe screeningsmethoden die deze toxische effecten al in een vroeg stadium van de ontwikkeling kunnen detecteren. Deze nieuwe screeningsmethoden zullen uiteindelijk leiden tot veiligere medicijnen en een efficiëntere ontwikkeling van nieuwe geneesmiddelen.

Genomica is de studie van de organisatie en de functies van het genoom van een cel of organisme. Hiervoor wordt de volledige genexpressie geanalyseerd van transcriptie van DNA naar mRNA (transcriptomica) en translatie van mRNA naar een functioneel eiwit (proteomica) tot aan de enzymatische productie van metabolieten (metabolomica).

Het gebruik van genomica om toxicologische processen te bestuderen heet toxicogenomica. Met toxicogenomica kunnen moleculaire mechanismen die gepaard gaan met de toxiciteit van bepaalde stoffen geïdentificeerd worden. De toxicologische eindpunten die gebruikt worden in de toxicogenomica kunnen zowel in vitro als in vivo, in proefdierstudies, bepaald worden. Daardoor kunnen in vivo metingen direct gelinkt worden met in vitro bepalingen. Deze vergelijkingen maken het mogelijk om in vitro modellen te ontwikkelen die lever toxische geneesmiddelen kunnen identificeren. Naast het identificeren van lever toxische stoffen is het ook belangrijk om deze stoffen te classificeren volgens het type levertoxiciteit dat ze veroorzaken. De meest voorkomende soorten levertoxiciteit zijn: 1) necrose, celdood veroorzaakt door vrije radicalen en toxische metabolieten; 2) steatose, opstapeling van kleine vetdruppels in de lever vaak veroorzaakt door mitochondriële schade waardoor vetzuren niet of slecht worden afgebroken; 3) cholestase; opstapeling van gal in de lever door een slechte werking van de gal transport eiwitten.

Het doel van dit proefschrift is om betrouwbare in vitro modellen te ontwikkelen om lever toxische stoffen te identificeren, te classificeren en een inzicht te krijgen in de moleculaire processen die deze stoffen teweegbrengen. Hiervoor werden gekende lever toxische (hepatotoxische) stoffen gebruikt die ofwel necrose, steatose of cholestase induceren. Aan de hand van proteoom analyse, gedeeltelijk verrijkt met transcriptomica data, werden expressieprofielen gegenereerd reflecterend voor de verschillende soorten van lever toxiciteit (necrose, cholestase en steatose).

\section{SAMENVATTING VAN DE STUDIES BESCHREVEN IN DIT PROEFSCHRIFT}

Voor het bestuderen van toxicologische processen wordt vaak gebruik gemaakt van HepG2 cellen. HepG2 is een onsterfelijke cellijn die gemaakt werd van humane levercellen afkomstig 
uit een hepatocellulair carcinoom. Deze cellen met lever-specifieke eigenschappen kunnen zichzelf oneindig vermenigvuldigen waardoor ze gemakkelijk te kweken zijn. In Hoofdstuk 2 werden HepG2 cellen bloodgesteld aan de hepatotoxische stoffen acetaminophen (induceert necrose), amiodarone (induceert steatose) en cyclosporine A (induceert cholestase). Daarna werd de eiwitexpressie in deze cellen bestudeerd. Aan de hand van de eiwitexpressie werden de moleculaire processen veroorzaakt door deze hepatotoxische stoffen geanalyseerd. Bovendien is onderzocht of deze hepatotoxische stoffen van elkaar onderscheiden kunnen worden op basis van hun eiwitexpressie profiel. Uit deze studie bleek dat het mogelijk was om cyclosporine A te onderscheiden van de controle en de HepG2 cellen behandeld met acetaminophen en amiodarone. Daarnaast werden er aanwijzingen gevonden dat cyclosporine $A$ een stressreactie veroorzaakt in het endoplasmatisch reticulum (ER). Een stressreactie van het ER kan onder andere veroorzaakt worden door opstapeling van cholesterol in het ER. Het is bekend dat cyclosporine $A$ het transport van galzouten uit de lever naar de galkanalen remt. Galzouten worden gemaakt uit cholesterol, de productie van gal is een van de manieren om de hoeveelheid cholesterol in de lever op peil te houden. Daarom zal cyclosporine A niet alleen zorgen voor een opstapeling van galzouten maar ook voor een stijging van de cholesterol, hetgeen vervolgens een stressreactie in het ER te weeg brengt.

Ondanks dat HepG2 cellen een veelgebruikt in vitro model is, hebben deze cellen ook een aantal nadelen. Zo missen ze een aantal lever specifieke enzymen die belangrijk zijn voor het metabolisme en de detoxificatie van toxische stoffen. Deze specifieke enzymen zijn wel aanwezig in primaire levercellen (hepatocyten) geïsoleerd uit humaan leverweefsel. Daarom wordt het gebruik van primaire humane hepatocyten aangeraden voor toxicologische onderzoeken. Echter, geschikt humaan leverweefsel om hepatocyten te isoleren is slechts in beperkte mate verkrijgbaar. Dit probleem kan deels worden opgelost door primaire muizen hepatocyten te gebruiken. In hoofdstuk 3 analyseerden we de eiwitexpressie van primaire muizen hepatocyten die werden blootgesteld aan acetaminophen, amiodarone of cyclosporine $A$. Bovendien werden deze resultaten vergeleken met de eiwitexpressie van HepG2 cellen, een in vitro model met humane cellen. Ook in primaire muizen hepatocyten kon het effect van cyclosporine A op het proteoom, onderscheiden worden van de controle en de, acetaminphen en amiodarone behandelde cellen. Vergelijkbaar met de HepG2 cellen bracht cyclosporine A ook in primaire muizen hepatocyten een stressreactie van het ER te weeg. Bovendien werden er aanwijzingen gevonden voor een belemmerd eiwittransport van het ER naar het Golgi-apparaat. ER-stress en een belemmerd ER-Golgi transport kan er toe leiden dat bepaalde eiwitten die normaal worden uitgescheiden, niet meer gesecreteerd kunnen worden en dus zullen opstapelen in de cel. Om deze hypothese verder te onderzoeken werd het secretoom (eiwitten uitgescheiden door de cellen) van primaire muizen hepatocyten na behandeling met acetaminophen, amiodarone of cyclosporine A geanalyseerd. Deze studie staat beschreven in hoofdstuk 4.

Het secretoom van de hepatocyten is een goede bron van kandidaat biomakers voor hepatotoxiciteit. Dit komt omdat, naast de detoxificatie van toxische stoffen, de lever ook verantwoordelijk is voor de productie en secretie van verschillende serumeiwitten. Met andere 
woorden, wanneer er specifieke eiwit markers voor hepatotoxiciteit worden gevonden in het secretoom van hepatocyten, is de kans groot dat deze markers ook aanwezig kunnen zijn in het bloed of serum van patiënten met levertoxiciteit. Hierdoor zou het mogelijk zijn om hepatotoxiciteit op te sporen bij patiënten, bijvoorbeeld in klinische studies.

Analyse van het secretoom van de primaire muizen hepatocyten toonde aan dat deze cellen een aanzienlijke hoeveelheid plasma-eiwitten uitscheiden, zoals albumine, ceruloplasmine en antitrombine III. Dit geeft aan dat in primaire muizen hepatocyten de lever-specifieke eigenschappen met betrekking tot eiwitsecretie goed bewaard zijn gebleven.

Op basis van de bevindingen uit hoofdstuk 3, veronderstelden we dat cyclosporine $A$ een stressreactie veroorzaakt in het ER, wat een remming van de eiwitsecretie zou veroorzaken. Het secretoom van primaire muizen hepatocyten behandeld met cyclosporine A vertoonde inderdaad een verminderde hoeveelheid van bepaalde eiwitten. Maar cyclosporine A verhoogde ook de secretie van een aantal plasma-eiwitten zoals serumalbumine. Sommige van deze plasmaeiwitten zijn ook verhoogd teruggevonden na blootstelling aan acetaminophen of amiodarone. Meestal gaat leverfalen gepaard met een verminderde hoeveelheid serumalbumine; een verhoogde aanwezigheid van serum eiwitten was dan ook onverwacht. Albumine is het meest voorkomende eiwitmolecuul in het bloed. Het is onder andere verantwoordelijk voor het transport van stoffen in het bloed. Niet alleen lichaamseigen stoffen maar ook lichaamsvreemde stoffen zoals medicijnen en toxicologische verbindingen. Waarschijnlijk is de verhoogde aanwezigheid van albumine in het secretoom van primaire muizen hepatocyten een beschermingsreactie in een vroeg stadium van toxiciteit. In een later stadium bij het optreden van leverfalen is de lever niet meer in staat om voldoende hoeveelheden albumine te produceren, wat zal resulteren in verlaagde hoeveelheid albumine in het bloed. Naast de differentiële expressie van typische plasmaeiwitten, hadden de lever toxische stoffen ook een invloed op het cholesterol- en lipidenmetabolisme. Veranderingen in het cholesterol- en lipidenmetabolisme zijn typisch voor het ziektebeeld van cholestase en steatose. Men zou daarom verwachten dat eiwitten die geassocieerd worden met het cholesterol- en lipidenmetabolism voornamelijk differentieel tot expressie worden gebracht door steatosis- en/of cholestase- inducerende verbindingen. Toch werden sommige van deze eiwitten ook door acetaminophen veranderd tot expressie gebracht. Dit wijst op een overlap van de moleculaire mechanismen geactiveerd door hepatotoxische stoffen die toch verschillende ziektebeelden teweegbrengen.

In de vorige studies werden HepG2 cellen en primaire muizen hepatocyten geëvalueerd op basis van een beperkte set eiwitten, geïdentificeerd met behulp van differentiële gel elektroforese (DIGE). Dankzij microarrays is het mogelijk om de expressie van duizenden genen te volgen. In hoofdstuk 5 werd de invloed van acetaminophen, amiodarone en cyclosporine A geanalyseerd op het niveau van genexpressie in HepG2 cellen en primaire muizen hepatocyten. Na de analyse van de genen die verschillend tot expressie werden gebracht door de hepatotoxische stoffen, bleek er een sterke gelijkenis te zijn tussen de toxische respons in 
HepG2 cellen en de reactie in primaire muizen hepatocyten. Dit wijst erop dat primaire muizen hepatocyten in staat zijn om toxische reacties in de mens te voorspellen.

In de voorgaande hoofdstukken werden alternatieve in vitro modellen (HepG2 en primaire muizen hepatocyten) geëvalueerd op hun vermogen om hepatotoxische stoffen te identificeren. Idealiter geven deze in vitro modellen een weerspiegeling van een toxicologische reactie in vivo. Daarom is het relevant om in vitro/in vivo vergelijkingen uit te voeren. In hoofdstuk 6 wordt de ontwikkeling van cholestase als gevolg van een dagelijkse dosis cyclosporine $A$ bestudeerd. Hiervoor werd de eiwit expressie in de levers van C57BL/6 muizen na 4, 11 en 25 dagen blootstelling aan cylcosporine A geanalyseerd. Na 25 dagen blootstelling konden typische kenmerken van cholestase waargenomen worden. Om een in vitro/in vivo vergelijking mogelijk te maken werd de eiwitexpressie na blootstelling van cyclosporine A aan de HepG2 cellen (hoofdstuk 2) en de primaire muizen hepatocyten (hoofdstuk 3) vergeleken met de eiwitexpressie geïnduceerd in vivo. Zowel in vitro als in vivo werd aangetoond dat cyclosporine $A$ een invloed heeft op het cholesterolmetabolisme, wat mogelijk kan leiden tot een stressreactie van het ER. Terwijl er in vitro aanwijzingen werden gevonden voor een stressreactie van het ER, waren deze effecten in vivo minder uitgesproken. In vivo werden voornamelijk de mitochondriën aangetast door cyclosporine A in plaats van het ER. Deze verschillen geven aan dat in vitro modellen verschillend kunnen reageren ten opzichte van de in vivo situatie. Mogelijk is dit te verklaren doordat in vitro de cellen in direct contact komen met de toxische stoffen. Bovendien functioneren in vitro de cellen onafhankelijk van andere cellen en organen. Deze verschillen tussen in vitro en in vivo modellen kunnen verklaren waarom het zo moeilijk is om in vitro biomarkers te valideren ten opzichtevan in vivo modellen. Ondanks sommige verschillen in de toxicologische respons in vitro ten op zichtte van in vivo, hebben de huidige in vitro modellen het potentieel om verschillende types van levertoxiciteit onderscheiden.

Tot nu toe werd voor het bestuderen van necrose, steatose en cholestase gebruik gemaakt van de levertoxische stoffen acetaminophen, amiodarone en cyclosporine A. In Hoofdstuk 7 wordt nagegaan of chemische stoffen die gelijkaardige toxicologische eindpunten (necrose, steatose of cholestase) veroorzaken, ook gelijkaardige eiwitprofielen laten zien. Hiervoor werden primaire muizen hepatocyten blootgesteld aan zes goed gekarakteriseerde hepatotoxische stoffen: diclofenac en paraquat (induceren necrose), tetracycline en valproic acid (induceren steatose) en chlorpramazine en ethenylestradiol (induceren cholestase). Twee stoffen die toxisch zijn voor de nieren: D-mannitol en $\mathrm{LiCO}_{3}$ werden gebruikt als negatieve controles. Daarna werd hun eiwitexpressie profiel geanalyseerd. Vervolgens werden deze resultaten gecombineerd met de resultaten uit hoofdstuk 3 , waar de primaire muis hepatocyten werden blootgesteld aan acetaminophen, amiodarone en cyclosporine A. Een principale componentenanalyse werd gebruikt om te bepalen of het mogelijk was om de verschillende soorten hepatotoxische stoffen van elkaar te onderscheiden op basis van hun eiwitexpressieprofiel. De necrotiserende verbindingen konden voornamelijk onderscheiden worden van de andere stoffen op basis van de volgende eiwitten: catalase, glutamaat pyruvaat transaminase 2 , UDP-glucose pyrophosphorylase 2, arginase 1 en peroxiredoxine 6 . Stoffen die cholesta- 
se induceren werden voornamelijk onderscheiden op basis van vitamine $D$ verbindend eiwit. Tot slot werden stoffen die steatose veroorzaken voornamelijk onderscheiden op basis van carnitine O-palmitoyltransferase 2. Daarom zijn deze eiwitten goede kandidaten voor klassespecifieke biomarkers, maar verdere validatie is nodig om hierover uitsluitsel te geven.

\section{TEKORTKOMINGEN EN AANBEVELINGEN}

Niet alle hepatotoxische stoffen bestudeerd in dit proefschrift, toonden een typisch toxicologisch effect op het proteoom van de geteste in vitro modellen. Mogelijk hebben deze in vitro modellen een verlaagde expressie van enzymen verantwoordelijk voor het metabolisme en de detoxificatie van toxische stoffen.

Verder wordt de classificeren van hepatoxische stoffen bemoeilijkt door een overlap van sommige fenotypische eigenschappen tussen de verschillende soorten van hepatotoxiciteit. Om verder inzicht te krijgen in de hepatoxische mechanismen is het aanbevolen om transcriptomica, proteomica en metabolomica data met elkaar te vergelijken. In dit proefschrift werd data van proteome analyse gedeeltelijk aangevuld met transcriptome data. Ondanks, de verschillende technieken uit de bio-informatica blijft het moeilijk om proteomica data te vergelijken met transcriptomica data. Met behulp van microarrays is het mogelijk om een bijna volledige set van target genen te analyseren, terwijl dit niet mogelijk is met de standaard technieken uit de proteomica. Proteome analyse met behulp van DIGE is vooral gericht op de detectie van oplosbare eiwitten zonder extreme pl waarden. Deze tekortkomingen kunnen gedeeltelijk worden overkomen door een DIGE analyse te combineren met vloeistofchromatografie gekoppeld met massa spectrometrie. Maar zelfs na het combineren van beide technieken is het aantal differentiële eiwitten die gedetecteerd kunnen worden met proteomics slechts een fractie van de data die met microarrays gegenereerd worden. In een directe vergelijking van proteome en transcriptome data zullen sterk genen die sterk tot uiting komen bevooroordeeld worden. In de toekomst, zal de ontwikkeling van nieuwe software de kracht van high-throughput technologieën uit de proteomica en transcriptomica versterken.

De in vitro modellen uit dit proefschrift hebben enkele beperkingen die de extrapolatie van toxicologische effecten in vitro naar humane in vivo effecten hinderen. Dit komt omdat de in vitro modellen uit dit proefschrift gebruik maken van slechts één soort celtype, namelijk hepatocyten. Terwijl in de lever ook nog andere soorten cellen voorkomen zoals Kupffer en stellate cellen. Dit zou kunnen voorkomen worden door gebruik te maken van in vitro modellen op basis van lever slices. In deze lever slices wordt de driedimensionale structuur behouden en bovendien bevatten ze de verschillende celtypes die voorkomen in de lever. Deze lever slices hebben echter als nadeel dat ze slechts voor een beperkte tijd in cultuur kunnen worden gehouden en de grote interindividuele variatie. Uiteindelijk zal verder onderzoek zal leiden tot het optimaliseren van de huidige in vitro modellen. 


\section{CONCLUSIE}

Het onderzoek uit dit proefschrift toont aan dat in vitro modellen op basis van hepatocyten relevant zijn voor het classificeren van gekende hepatotoxische stoffen. De studies uit dit proefschrift tonen aan dat proteoom analyse van zowel cellulaire als gesecreteerde eiwitten toxicologische mechanismen aan het licht kan brengen.

Een vergelijking van de gen- en eiwitexpressie profielen, geïnduceerd door hepatotoxische stoffen in HepG2 toonde sterke overeenkomsten met de toxicologische reacties in primaire muizen hepatocyten.

Een in vitro/in vivo vergelijking van de eiwit expressie geïnduceerd door cyclosporine $A$, toonde overeenkomsten in ATP-binding, en cholesterol en koolhydraat metabolisme. Ondanks deze overeenkomstige reacties, werden er ook verschillen gevonden tussen de in vitro en de in vivo response. Zo werden in vivo voornamelijk de mitochondriën aangetast, terwijl in vitro voornamelijk het ER werd aangevallen. Deze verschillen in toxicologische reacties tussen in vivo en in vitro modellen kunnen de validatie van in vitro biomarkers in in vivo modellen bemoeilijken.

Desondanks werd aangetoond dat de huidige in vitro modellen het potentieel hebben om hepatoxische stoffen te identificeren en ze in te delen volgens het type hepatotoxiciteit dat ze veroorzaken. Daarmee vormt dit onderzoek een basis voor de verdere ontwikkeling van in vitro modellen om hepatotoxische stoffen te identificeren en te classificeren met behulp van toxicogenomica. 
Supplemental data 


\section{SUPPLEMENTAL DATA}

\section{Supplemental data chapter 5}

A

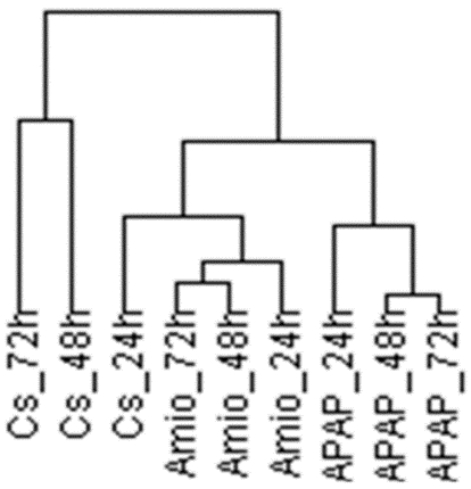

B

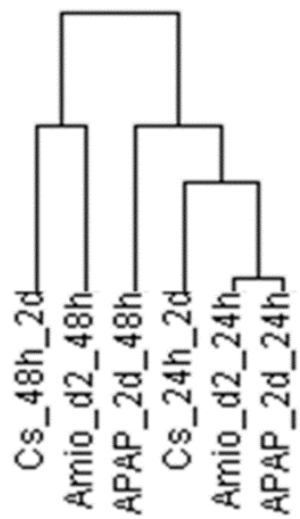

Supplemental Figure 5.1: Hierarchical clustering of differentially expressed mRNA in HepG2 and primary mouse hepatocytes (PMH) cells after acetaminophen (APAP), cyclosporin A (CsA) and amiodarone (Amio) exposure across time. (A) Data set in which at least 6 of 9 conditions in HepG2 were significant. (B) Data set in which at least 2 of 6 conditions in PMH were significant. The differentially expressed genes (Fold change $\geq 1.5$; $\mathrm{p}$-value $<0.05$ ) upon 9 compounds exposure showed improved clusters at $48 \mathrm{~h}$.

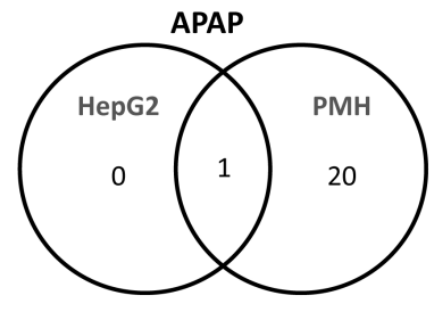

1. Extracellular space

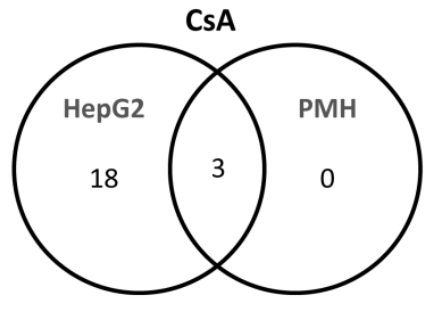

1. Lipid transport activity

2. Extracellular space

3. very-low-density lipoprotein particle
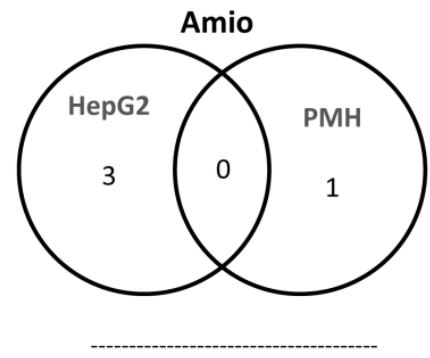
Supplemental Table 5.1: Overview of the differentially expressed genes in HepG2 and primary mouse hepatocytes across time after the exposure to acetaminophen (APAP), cyclosporin A ( CsA) and amiodarone (Amio) across time.

HepG2

\begin{tabular}{|c|c|c|c|c|c|c|c|c|}
\hline APAP $24 h$ & APAP $48 \mathrm{~h}$ & APAP $24 \mathrm{~h}$ & CsA 24h & CsA 48h & CsA 72h & Amio $24 \mathrm{~h}$ & Amio $48 \mathrm{~h}$ & Amio $72 \mathrm{~h}$ \\
\hline 2279 & 2595 & 2819 & 3145 & 3975 & 3528 & 34 & 121 & 276 \\
\hline $1238 \uparrow \quad 1041 \downarrow$ & $1393 \uparrow \quad 1202 \downarrow$ & $1536 \uparrow \quad 1283 \downarrow$ & $1309 \uparrow \quad 1836 \downarrow$ & $1777 \uparrow \quad 1298 \downarrow$ & $1682 \uparrow \quad 1846 \downarrow$ & $25 \downarrow$ & $77 \downarrow$ & $125 \uparrow \quad 151 \downarrow$ \\
\hline
\end{tabular}

Primary mouse hepatocytes

\begin{tabular}{|c|c|c|c|c|c|}
\hline APAP $24 \mathrm{~h}$ & APAP $48 \mathrm{~h}$ & CsA $24 \mathrm{~h}$ & CsA 48h & Amio $24 \mathrm{~h}$ & Amio $48 \mathrm{~h}$ \\
\hline 1773 & 2037 & 3594 & 3799 & 1421 & 2980 \\
\hline $788 \uparrow \quad 985 \downarrow$ & $847 \uparrow \quad 1190 \downarrow$ & $1789 \uparrow \quad 1805 \downarrow$ & $1837 \uparrow \quad 1962 \downarrow$ & $655 \uparrow 766 \downarrow$ & $1324 \uparrow \quad 1656 \downarrow$ \\
\hline
\end{tabular}

$\uparrow / \downarrow=$ Upregulated/Downregulated differentially expressed genes.

Supplemental Table 5.2: Conserved GO Processes in both HepG2 cells and primary mouse hepatocytes (PMH) after exposure acetaminophen (APAP), cyclosporin A (CsA) and amiodarone (Amio) exposure, per time point.

\begin{tabular}{|c|c|c|c|c|c|c|c|c|c|c|c|c|c|c|c|}
\hline \multirow{3}{*}{ GO Processes } & \multicolumn{9}{|c|}{ HepG2 cells (t-values) } & \multicolumn{6}{|c|}{ Primary mouse hepatocytes ( $t$-values) } \\
\hline & APAP & APAP & APAP & Amio & Amio & Amio & CsA & CsA & CsA & APAP & APAP & Amio & Amio & CsA & CsA \\
\hline & $24 \mathrm{~h}$ & $48 \mathrm{~h}$ & $72 \mathrm{~h}$ & $24 \mathrm{~h}$ & $48 \mathrm{~h}$ & $72 \mathrm{~h}$ & $24 \mathrm{~h}$ & $48 \mathrm{~h}$ & $72 \mathrm{~h}$ & $24 \mathrm{~h}$ & $48 \mathrm{~h}$ & $24 \mathrm{~h}$ & $48 \mathrm{~h}$ & $24 \mathrm{~h}$ & $48 \mathrm{~h}$ \\
\hline $\begin{array}{l}\text { lipid transporter } \\
\text { activity }\end{array}$ & -2.89 & -2.51 & -1.71 & -0.65 & -1.08 & -1.57 & -5.68 & -4.35 & -5.12 & -3.1 & -0.66 & -0.87 & -3.71 & -2.26 & -4.35 \\
\hline extracellular space & -4.01 & -4.24 & -2.87 & -2.56 & 0.74 & -3.17 & -14.71 & -4.64 & -7.36 & -6.54 & -3.3 & -1.59 & -2.74 & -3.3 & -4.15 \\
\hline $\begin{array}{l}\text { very-low-density } \\
\text { lipoprotein particle }\end{array}$ & -3.27 & -3.55 & -2.64 & 0.78 & 0.47 & -0.18 & -5.87 & -4.39 & -5.46 & -1.87 & -0.64 & -1.27 & -4.74 & -2.49 & -4.97 \\
\hline
\end{tabular}

Positive and negative t-values indicate predominant up- and down-regulation of genes within the term. All the significant GO terms highlighted in this table have an e-value (Bonferroni corrected $p$-value) below 0.05 and $\mid t$-value $\mid \geq 4$. 


\section{Supplemental data chapter 7}

Supplemental Table 7.1: Protein identification of differentially expressed proteins in primary mouse hepatocytes after exposure to chlorpromazine (Chlor), ethinylestradiol $(\mathrm{EE})$, tetracycline $(\mathrm{TC})$, valproic acid $(\mathrm{VA})$, paraquat $(\mathrm{PQ})$, negative controls D-mannitol (Dm) and $\mathrm{LiCO}_{3}$.

\begin{tabular}{|c|c|c|c|c|c|c|c|c|c|c|c|}
\hline \multirow{3}{*}{$\begin{array}{l}\text { Spot } \\
\text { No. }\end{array}$} & \multirow{3}{*}{$\begin{array}{l}\text { Uniprot } \\
\text { Acces- } \\
\text { sion No. }\end{array}$} & \multirow{3}{*}{$\begin{array}{l}\text { Gene } \\
\text { name }\end{array}$} & \multirow[t]{3}{*}{ Protein Description } & \multirow{3}{*}{$\begin{array}{l}\text { P-value } \\
\text { (one-way } \\
\text { ANOVA) }\end{array}$} & \multicolumn{7}{|c|}{ Fold Change } \\
\hline & & & & & \multicolumn{2}{|c|}{ Cholestasis } & \multicolumn{2}{|c|}{ Steatosis } & \multirow{2}{*}{$\begin{array}{l}\text { Necrosis } \\
\text { PBS/PQ }\end{array}$} & \multicolumn{2}{|c|}{$\begin{array}{l}\text { Negative } \\
\text { Controls }\end{array}$} \\
\hline & & & & & $\begin{array}{l}\text { DMSO/ } \\
\text { Chlor }\end{array}$ & $\begin{array}{l}\text { DMSO/ } \\
\text { EE }\end{array}$ & $\begin{array}{l}\text { DMSO/ } \\
\text { TC }\end{array}$ & PBS/VA & & $\begin{array}{l}\text { DMSO } \\
\text { /Dm }\end{array}$ & $\begin{array}{l}\mathrm{PBS} / \\
\mathrm{LiCO}_{3}\end{array}$ \\
\hline 1 & Q05920 & Pc & Pyruvate carboxylase, mitochondrial precursor & $8.39 \mathrm{E}-03$ & -1.10 & -1.11 & -1.08 & 1.22 & -1.18 & -1.01 & -1.02 \\
\hline 2 & Q78PY7 & Snd1 & $\begin{array}{l}\text { Staphylococcal nuclease domain-containing protein } \\
1\end{array}$ & $4.20 \mathrm{E}-03$ & 1.16 & 1.30 & 1.06 & $1.39^{* *}$ & 1.03 & 1.12 & 1.08 \\
\hline 3 & P08113 & Hsp90b1 & Endoplasmin & $2.40 \mathrm{E}-04$ & 1.06 & 1.20 & $1.03^{* *}$ & 1.49 & -1.13 & 1.09 & 1.16 \\
\hline 4 & P57780 & Actn4 & Alpha-actinin 4 (Non-muscle alpha-actinin 4) & $3.10 \mathrm{E}-03$ & 1.02 & 1.05 & -1.02 & 1.30 & -1.20 & 1.02 & -1.04 \\
\hline 5 & P28271 & Aco1 & Iron-responsive element binding protein 1 & $2.40 \mathrm{E}-07$ & -1.14 & -1.08 & -1.20 & -1.10 & -1.06 & 1.05 & 1.08 \\
\hline 6 & Q01853 & Vcp & Transitional endoplasmic reticulum ATPase & $5.70 \mathrm{E}-03$ & 1.02 & -1.00 & -1.02 & -1.06 & -1.50 & 1.08 & -1.01 \\
\hline 7 & P07901 & Hsp90aa1 & Heat shock protein HSP 90-alpha (HSP 86) & $4.90 \mathrm{E}-05$ & $1.09^{* *}$ & $1.04^{* *}$ & 1.02 & -1.23 & -1.08 & 1.03 & 1.03 \\
\hline 8 & Q99KI0 & Aco2 & Aconitate hydratase, mitochondrial precursor & $9.90 \mathrm{E}-03$ & 1.00 & -1.03 & -1.00 & -1.02 & 1.06 & -1.02 & -1.09 \\
\hline 9 & Q99KI0 & Aco2 & Aconitate hydratase, mitochondrial precursor & 7.70E-03 & 1.08 & 1.03 & 1.02 & 1.08 & -1.11 & 1.03 & -1.02 \\
\hline 10 & Q99KI0 & Aco2 & Aconitate hydratase, mitochondrial precursor & $5.60 \mathrm{E}-06$ & 1.03 & 1.01 & -1.08 & 1.20 & -1.20 & -1.01 & -1.05 \\
\hline 11 & Q8CAQ8 & Immt & Mitochondrial inner membrane protein & $3.30 \mathrm{E}-02$ & 1.06 & 1.13 & -1.03 & $1.32^{* *}$ & 1.18 & 1.07 & 1.14 \\
\hline 12 & Q9CZD3 & Gars & Glycyl-tRNA synthetase & $3.90 \mathrm{E}-06$ & 1.03 & 1.10 & -1.00 & $1.42^{* *}$ & 1.05 & 1.06 & 1.08 \\
\hline 13 & P48678 & Lmna & Prelamin-A/C & $1.60 \mathrm{E}-02$ & $1.09^{*}$ & -1.06 & 1.19 & -1.48 & -1.13 & -1.08 & -1.11 \\
\hline 14 & P97494 & Gclc & Glutamate--cysteine ligase catalytic subunit & 3.10E-03 & 1.23 & 1.10 & -1.05 & 1.02 & 1.06 & -1.03 & 1.04 \\
\hline 15 & P41216 & Acsl1 & Long-chain-fatty-acid--CoA ligase 1 & $4.30 \mathrm{E}-04$ & -1.07 & -1.13 & -1.12 & $1.10^{* *}$ & 1.00 & -1.12 & 1.09 \\
\hline 16 & P63017 & Hspa8 & Heat shock cognate $71 \mathrm{kDa}$ protein & $5.40 \mathrm{E}-03$ & -1.03 & 1.11 & 1.10 & 1.46 & 1.10 & -1.02 & 1.05 \\
\hline 17 & P63017 & Hspa8 & Heat shock cognate $71 \mathrm{kDa}$ protein & $8.50 \mathrm{E}-03$ & 1.15 & 1.15 & -1.05 & -1.11 & 1.43 & -1.02 & 1.09 \\
\hline
\end{tabular}




\begin{tabular}{|c|c|c|c|c|c|c|c|c|c|c|c|}
\hline \multirow{3}{*}{$\begin{array}{l}\text { Spot } \\
\text { No. }\end{array}$} & \multirow{3}{*}{$\begin{array}{l}\text { Uniprot } \\
\text { Acces- } \\
\text { sion No. }\end{array}$} & \multirow{3}{*}{$\begin{array}{l}\text { Gene } \\
\text { name }\end{array}$} & \multirow[t]{3}{*}{ Protein Description } & \multirow{3}{*}{$\begin{array}{l}\text { P-value } \\
\text { (one-way } \\
\text { ANOVA) }\end{array}$} & \multicolumn{7}{|c|}{ Fold Change } \\
\hline & & & & & \multicolumn{2}{|c|}{ Cholestasis } & \multicolumn{2}{|c|}{ Steatosis } & \multirow[t]{2}{*}{ Necrosis } & \multicolumn{2}{|c|}{$\begin{array}{l}\text { Negative } \\
\text { Controls }\end{array}$} \\
\hline & & & & & $\begin{array}{l}\text { DMSO/ } \\
\text { Chlor }\end{array}$ & $\begin{array}{l}\text { DMSO/ } \\
\text { EE }\end{array}$ & $\begin{array}{l}\text { DMSO/ } \\
\text { TC }\end{array}$ & PBS/VA & & $\begin{array}{l}\text { DMSO } \\
\text { /Dm }\end{array}$ & $\begin{array}{l}\mathrm{PBS} / \\
\mathrm{LiCO}_{3}\end{array}$ \\
\hline 18 & P07724 & Alb & Serum albumin & $3.50 \mathrm{E}-02$ & 1.14 & 1.15 & -1.07 & -1.06 & 1.21 & -1.00 & 1.04 \\
\hline 19 & P07724 & Alb & Serum albumin & $2.10 \mathrm{E}-02$ & -1.23 & -1.19 & -1.42 & 1.31 & -1.11 & -1.05 & 1.03 \\
\hline 20 & P07724 & Alb & Serum albumin & $1.60 \mathrm{E}-05$ & -1.06 & -1.03 & $1.06^{* *}$ & $-1.10^{* *}$ & 1.01 & -1.00 & -1.02 \\
\hline 21 & P07724 & Alb & Serum albumin & $2.20 \mathrm{E}-04$ & 1.04 & 1.02 & $-1.11^{* *}$ & 1.03 & -1.00 & -1.03 & 1.08 \\
\hline 22 & P40142 & Tkt & Transketolase & $6.50 \mathrm{E}-03$ & -1.08 & -1.10 & -1.12 & 1.35 & -1.05 & -1.03 & 1.00 \\
\hline 23 & Q9Z0X1 & Aifm1 & Apoptosis-inducing factor 1 & $8.00 \mathrm{E}-03$ & -1.03 & 1.03 & -1.07 & $-1.05^{* *}$ & -1.16 & -1.04 & 1.08 \\
\hline 24 & Q8VCU1 & Gm4738 & Liver carboxylesterase 31-like & $9.20 \mathrm{E}-03$ & 1.12 & $1.09^{* *}$ & -1.06 & 1.34 & 1.13 & -1.03 & -1.06 \\
\hline 25 & Q9Z0X1 & Aifm1 & Apoptosis-inducing factor 1 , mitochondrial & $3.90 \mathrm{E}-06$ & -1.17 & -1.61 & -1.10 & $-1.06^{* *}$ & -1.17 & -1.07 & -1.05 \\
\hline 26 & P24527 & Lta4h & Leukotriene $\mathrm{A}-4$ hydrolase & $3.10 \mathrm{E}-02$ & -1.01 & 1.02 & -1.01 & 1.36 & 1.02 & -1.05 & -1.02 \\
\hline 27 & P52825 & Cpt2 & Carnitine O-palmitoyltransferase 2, mitochondrial & 7.90E-03 & 1.03 & 1.00 & 1.19 & $1.13^{* *}$ & -1.09 & 1.09 & 1.02 \\
\hline 28 & P50544 & Acadvl & $\begin{array}{l}\text { Very long-chain specific acyl-CoA dehydrogenase, } \\
\text { mitochondrial }\end{array}$ & $3.90 \mathrm{E}-05$ & -1.00 & -1.01 & 1.01 & $1.31^{* *}$ & 1.05 & -1.05 & 1.04 \\
\hline 29 & Q8QZR3 & Ces2a & Carboxylesterase 6 & $1.10 \mathrm{E}-02$ & -1.01 & $-1.04^{*}$ & -1.03 & -1.09 & -1.06 & -1.01 & 1.05 \\
\hline 30 & Q8VCW8 & Acsf2 & $\begin{array}{l}\text { Acyl-CoA synthetase family member } 2 \text {, mitochon- } \\
\text { drial }\end{array}$ & $4.70 \mathrm{E}-03$ & 1.02 & 1.02 & 1.11 & $-1.09^{*}$ & -1.02 & 1.07 & 1.01 \\
\hline 31 & Q8VCW8 & Acsf2 & $\begin{array}{l}\text { Acyl-CoA synthetase family member } 2 \text {, mitochon- } \\
\text { drial }\end{array}$ & $9.60 \mathrm{E}-07$ & 1.08 & 1.01 & 1.11 & $-1.02^{* *}$ & 1.03 & 1.01 & -1.04 \\
\hline 32 & Q8VCW8 & Acsf2 & $\begin{array}{l}\text { Acyl-CoA synthetase family member } 2 \text {, mitochon- } \\
\text { drial }\end{array}$ & $3.50 \mathrm{E}-05$ & -1.02 & -1.06 & 1.01 & $-1.54^{* *}$ & -1.21 & 1.02 & 1.02 \\
\hline 33 & P27773 & Pdia3 & Protein disulfide-isomerase $\mathrm{A} 3$ & $2.40 \mathrm{E}-03$ & 1.10 & 1.04 & 1.10 & $-1.40^{* *}$ & -1.11 & 1.05 & -1.01 \\
\hline 34 & Q00612 & G6pdx & Glucose-6-phosphate 1-dehydrogenase X & $3.90 \mathrm{E}-05$ & -1.07 & $-1.24^{*}$ & 1.12 & $-1.30^{* *}$ & -1.02 & -1.08 & -1.12 \\
\hline 35 & P21614 & $\mathrm{Gc}$ & Vitamin D-binding protein & $4.90 \mathrm{E}-03$ & 1.47 & 1.20 & 1.00 & $-1.07^{* *}$ & 1.20 & 1.10 & 1.13 \\
\hline 36 & Q9JMH6 & Txnrd1 & Isoform 2 of Thioredoxin reductase 1 , cytoplasmic & $1.30 \mathrm{E}-02$ & 1.08 & 1.05 & 1.17 & 1.33 & $1.37^{*}$ & -1.06 & 1.01 \\
\hline
\end{tabular}




\begin{tabular}{|c|c|c|c|c|c|c|c|c|c|c|c|}
\hline \multirow{3}{*}{$\begin{array}{l}\text { Spot } \\
\text { No. }\end{array}$} & \multirow{3}{*}{$\begin{array}{l}\text { Uniprot } \\
\text { Acces- } \\
\text { sion No. }\end{array}$} & \multirow{3}{*}{$\begin{array}{l}\text { Gene } \\
\text { name }\end{array}$} & \multirow[t]{3}{*}{ Protein Description } & \multirow{3}{*}{$\begin{array}{l}\text { P-value } \\
\text { (one-way } \\
\text { ANOVA) }\end{array}$} & \multicolumn{7}{|c|}{ Fold Change } \\
\hline & & & & & \multicolumn{2}{|c|}{ Cholestasis } & \multicolumn{2}{|c|}{ Steatosis } & \multirow[t]{2}{*}{ Necrosis } & \multicolumn{2}{|c|}{$\begin{array}{l}\text { Negative } \\
\text { Controls }\end{array}$} \\
\hline & & & & & $\begin{array}{l}\text { DMSO/ } \\
\text { Chlor }\end{array}$ & $\begin{array}{l}\text { DMSO/ } \\
\text { EE }\end{array}$ & $\begin{array}{l}\text { DMSO/ } \\
\text { TC }\end{array}$ & PBS/VA & & $\begin{array}{l}\text { DMSO } \\
\text { /Dm }\end{array}$ & $\begin{array}{l}\mathrm{PBS} / \\
\mathrm{LiCO}_{3}\end{array}$ \\
\hline 37 & $\mathrm{P} 24270$ & Cat & Catalase & $2.00 \mathrm{E}-03$ & 1.04 & 1.14 & 1.06 & -1.11 & $1.29^{* *}$ & -1.02 & -1.07 \\
\hline 38 & P24270 & Cat & Catalase & $2.80 \mathrm{E}-03$ & -1.18 & -1.11 & -1.09 & $-1.27^{* *}$ & 1.02 & -1.05 & -1.06 \\
\hline 39 & P11679 & Krt8 & Keratin, type II cytoskeletal 8 & $6.50 \mathrm{E}-03$ & $1.55^{* *}$ & 1.21 & 1.20 & -1.06 & 1.21 & 1.05 & 1.07 \\
\hline 40 & Q8BGT5 & Gpt2 & Alanine aminotransferase 2 & $4.90 \mathrm{E}-03$ & -1.14 & 1.00 & -1.00 & $-1.41^{*}$ & -1.35 & 1.11 & 1.02 \\
\hline 41 & Q91ZJ5 & Ugp2 & UTP--glucose-1-phosphate uridylyltransferase & $1.70 \mathrm{E}-02$ & 1.08 & -1.03 & -1.13 & -1.37 & 1.07 & 1.09 & 1.06 \\
\hline 42 & Q91ZJ5 & Ugp2 & UTP--glucose-1-phosphate uridylyltransferase & $6.50 \mathrm{E}-03$ & -1.02 & -1.06 & -1.18 & $-1.33^{* *}$ & 1.01 & 1.04 & 1.06 \\
\hline 43 & 009173 & Hgd & Homogentisate 1,2-dioxygenase & $1.90 \mathrm{E}-03$ & -1.09 & -1.08 & -1.13 & -1.04 & $-1.26^{* *}$ & -1.02 & 1.03 \\
\hline 44 & P54869 & Hmgcs2 & Hydroxymethylglutaryl-CoA synthase, mitochondrial & 7.00E-03 & -1.03 & -1.05 & 1.07 & $-1.30^{* *}$ & -1.03 & 1.05 & -1.02 \\
\hline 45 & P51855 & Gss & Glutathione synthetase & $8.50 \mathrm{E}-03$ & -1.05 & -1.09 & $-1.06^{*}$ & -1.13 & -1.15 & -1.05 & -1.01 \\
\hline 46 & P46471 & Psmc2 & 265 protease regulatory subunit 7 & $8.40 \mathrm{E}-03$ & 1.27 & 1.07 & 1.02 & 1.11 & $1.46^{* *}$ & 1.13 & 1.28 \\
\hline 47 & P17182 & Eno1 & Alpha-enolase & $1.80 \mathrm{E}-02$ & 1.14 & 1.07 & -1.20 & -1.16 & 1.22 & 1.01 & 1.04 \\
\hline 48 & P05784 & Krt18 & Keratin, type I cytoskeletal 18 & $2.60 \mathrm{E}-03$ & -1.37 & -1.39 & -1.04 & $-1.64^{* *}$ & -1.24 & -1.09 & -1.28 \\
\hline 49 & P17182 & Eno1 & Alpha-enolase & 7.00E-04 & 1.05 & -1.01 & $-1.19^{*}$ & -1.07 & -1.03 & 1.02 & 1.13 \\
\hline 50 & P55264 & Adk & Adenosine kinase & $2.80 \mathrm{E}-03$ & -1.11 & -1.09 & $-1.20^{* *}$ & -1.05 & -1.06 & 1.01 & 1.10 \\
\hline 51 & P60710 & Actb & Actin, cytoplasmic 1 & $3.20 \mathrm{E}-02$ & -1.02 & -1.14 & -1.17 & $-1.35^{* *}$ & -1.14 & -1.04 & -1.10 \\
\hline 52 & P60710 & Actb & Actin, cytoplasmic 1 & $3.60 \mathrm{E}-04$ & -1.11 & 1.23 & $-1.12^{*}$ & $1.33^{* *}$ & -1.15 & -1.13 & 1.08 \\
\hline 53 & P11679 & Krt8 & Keratin, type II cytoskeletal 8 & $5.00 \mathrm{E}-02$ & -1.79 & -1.69 & -1.31 & $-2.46^{*}$ & -1.30 & -1.01 & -1.36 \\
\hline 54 & Q07417 & Acads & $\begin{array}{l}\text { Short-chain specific acyl-CoA dehydrogenase, } \\
\text { mitochondrial }\end{array}$ & $1.90 \mathrm{E}-02$ & 1.01 & -1.01 & 1.05 & 1.13 & $1.25^{*}$ & 1.02 & -1.05 \\
\hline 55 & Q61176 & Arg1 & Arginase-1 & $1.30 \mathrm{E}-02$ & -1.01 & 1.07 & -1.11 & -1.18 & 1.30 & 1.09 & 1.02 \\
\hline 56 & Q61176 & Arg1 & Arginase-1 & $3.10 \mathrm{E}-05$ & 1.02 & 1.15 & -1.02 & 1.16 & $1.53^{* *}$ & 1.06 & 1.09 \\
\hline 57 & Q61176 & Arg1 & Arginase-1 & $4.40 \mathrm{E}-02$ & -1.12 & -1.06 & -1.25 & -1.12 & -1.11 & 1.03 & 1.07 \\
\hline 58 & Q64442 & Sord & Sorbitol dehydrogenase & $1.00 \mathrm{E}-06$ & 1.13 & 1.15 & 1.00 & -1.15 & $-1.85^{* *}$ & 1.12 & 1.15 \\
\hline
\end{tabular}




\begin{tabular}{|c|c|c|c|c|c|c|c|c|c|c|c|}
\hline \multirow{3}{*}{$\begin{array}{l}\text { Spot } \\
\text { No. }\end{array}$} & \multirow{3}{*}{$\begin{array}{l}\text { Uniprot } \\
\text { Acces- } \\
\text { sion No. }\end{array}$} & \multirow{3}{*}{$\begin{array}{l}\text { Gene } \\
\text { name }\end{array}$} & \multirow[t]{3}{*}{ Protein Description } & \multirow{3}{*}{$\begin{array}{l}\text { P-value } \\
\text { (one-way } \\
\text { ANOVA) }\end{array}$} & \multicolumn{7}{|c|}{ Fold Change } \\
\hline & & & & & \multicolumn{2}{|c|}{ Cholestasis } & \multicolumn{2}{|c|}{ Steatosis } & \multirow[t]{2}{*}{ Necrosis } & \multicolumn{2}{|c|}{$\begin{array}{l}\text { Negative } \\
\text { Controls }\end{array}$} \\
\hline & & & & & $\begin{array}{l}\text { DMSO/ } \\
\text { Chlor }\end{array}$ & $\begin{array}{l}\text { DMSO/ } \\
\text { EE }\end{array}$ & $\begin{array}{l}\text { DMSO/ } \\
\text { TC }\end{array}$ & PBS/VA & & $\begin{array}{l}\text { DMSO } \\
\text { /Dm }\end{array}$ & $\begin{array}{l}\mathrm{PBS} / \\
\mathrm{LiCO}_{3}\end{array}$ \\
\hline 59 & P11679 & Krt8 & Keratin, type II cytoskeletal 8 & $6.90 \mathrm{E}-04$ & -2.18 & -1.89 & -1.75 & $-2.75^{* *}$ & 1.12 & 1.17 & 1.10 \\
\hline 60 & P60710 & Actb & Actin, cytoplasmic 1 & $6.60 \mathrm{E}-04$ & -1.60 & -1.82 & -1.83 & $-2.02^{* *}$ & -1.23 & -1.04 & 1.17 \\
\hline 61 & P05784 & Krt18 & Keratin, type I cytoskeletal 18 & $4.80 \mathrm{E}-03$ & -1.56 & -1.53 & -1.50 & -1.64 & 1.13 & 1.05 & 1.06 \\
\hline 62 & Q61176 & Arg1 & Arginase-1 & $6.00 \mathrm{E}-07$ & -1.07 & -1.03 & -1.08 & $1.23^{* *}$ & $1.86^{* *}$ & -1.02 & -1.01 \\
\hline 63 & Q61176 & Arg1 & Arginase-1 & 4.70E-03 & -1.16 & -1.08 & -1.27 & -1.25 & -1.18 & 1.02 & 1.06 \\
\hline 64 & P05784 & Krt18 & Keratin, type I cytoskeletal 18 & $1.10 \mathrm{E}-03$ & -2.30 & -1.83 & -1.94 & $-2.70^{* *}$ & 1.10 & 1.05 & 1.19 \\
\hline 65 & P16858 & Gapdh & Glyceraldehyde-3-phosphate dehydrogenase & $1.80 \mathrm{E}-02$ & 1.29 & 1.02 & 1.04 & 1.20 & 1.01 & 1.07 & -1.00 \\
\hline 66 & Q9D819 & Ppa1 & Inorganic pyrophosphatase & $3.20 \mathrm{E}-02$ & 1.10 & 1.13 & -1.04 & 1.00 & 1.29 & 1.02 & 1.11 \\
\hline 67 & P08249 & Mdh2 & Malate dehydrogenase, mitochondrial & $2.20 \mathrm{E}-03$ & -1.05 & -1.05 & 1.02 & $1.36^{* *}$ & 1.02 & -1.10 & 1.07 \\
\hline 68 & P14152 & Mdh1 & Malate dehydrogenase, cytoplasmic & $3.80 \mathrm{E}-02$ & -1.23 & -1.05 & -1.20 & 1.14 & 1.34 & -1.22 & 1.18 \\
\hline 69 & P48036 & Anxa5 & Annexin A5 & $8.90 \mathrm{E}-03$ & 1.31 & 1.13 & -1.04 & -1.25 & -1.13 & 1.01 & 1.15 \\
\hline 70 & Q78JT3 & Haao & 3-hydroxyanthranilate 3,4-dioxygenase & $1.70 \mathrm{E}-05$ & 1.05 & 1.12 & -1.00 & -1.09 & $-1.47^{* *}$ & -1.01 & 1.03 \\
\hline 71 & Q60932 & Vdac1 & $\begin{array}{l}\text { Voltage-dependent anion-selective channel protein } \\
1\end{array}$ & $2.30 \mathrm{E}-02$ & 1.04 & 1.04 & 1.19 & $1.46^{* *}$ & 1.10 & 1.05 & 1.06 \\
\hline 72 & Q99KR3 & Lactb2 & Beta-lactamase-like protein 2 & $2.90 \mathrm{E}-04$ & -1.00 & -1.02 & -1.14 & -1.10 & $-1.86^{* *}$ & -1.00 & 1.14 \\
\hline 73 & P67778 & Phb & Prohibitin & $1.70 \mathrm{E}-02$ & -1.07 & -1.15 & -1.12 & 1.07 & -1.10 & -1.09 & -1.07 \\
\hline 74 & Q99P30 & Nudt7 & $\begin{array}{l}\text { Isoform } 1 \text { of Peroxisomal coenzyme A diphospha- } \\
\text { tase NUDT7 }\end{array}$ & $1.40 \mathrm{E}-02$ & 1.05 & 1.13 & $1.43^{* *}$ & -1.04 & 1.06 & 1.07 & -1.08 \\
\hline 75 & P17751 & Tpi1 & Triosephosphate isomerase & $2.80 \mathrm{E}-04$ & -1.05 & -1.08 & -1.14 & 1.31 & 1.03 & -1.19 & 1.04 \\
\hline 76 & P17751 & Tpi1 & Triosephosphate isomerase & $3.80 \mathrm{E}-02$ & -1.06 & 1.06 & 1.06 & -1.05 & 1.26 & -1.08 & 1.10 \\
\hline 76 & 008709 & Prdx6 & Peroxiredoxin- 6 & $3.80 \mathrm{E}-02$ & -1.06 & 1.06 & 1.06 & -1.05 & 1.26 & -1.08 & 1.10 \\
\hline 77 & 008807 & Prdx4 & Peroxiredoxin-4 & $4.60 \mathrm{E}-04$ & -1.22 & $-1.31^{* *}$ & -1.16 & $-1.37^{* *}$ & -1.16 & -1.09 & -1.05 \\
\hline 78 & P19639 & Gstm3 & Glutathione S-transferase Mu 3 & $6.30 \mathrm{E}-04$ & -1.06 & 1.13 & -1.22 & -1.36 & $-2.11^{* *}$ & -1.07 & 1.02 \\
\hline
\end{tabular}




\begin{tabular}{|c|c|c|c|c|c|c|c|c|c|c|c|}
\hline \multirow{3}{*}{$\begin{array}{l}\text { Spot } \\
\text { No. }\end{array}$} & \multirow{3}{*}{$\begin{array}{l}\text { Uniprot } \\
\text { Acces- } \\
\text { sion No. }\end{array}$} & \multirow{3}{*}{$\begin{array}{l}\text { Gene } \\
\text { name }\end{array}$} & \multirow[t]{3}{*}{ Protein Description } & \multirow{3}{*}{$\begin{array}{l}\text { P-value } \\
\text { (one-way } \\
\text { ANOVA) }\end{array}$} & \multicolumn{7}{|c|}{ Fold Change } \\
\hline & & & & & \multicolumn{2}{|c|}{ Cholestasis } & \multicolumn{2}{|l|}{ Steatosis } & \multirow{2}{*}{$\begin{array}{l}\text { Necrosis } \\
\text { PBS/PQ }\end{array}$} & \multicolumn{2}{|c|}{\begin{tabular}{|l|} 
Negative \\
Controls
\end{tabular}} \\
\hline & & & & & $\begin{array}{l}\text { DMSO/ } \\
\text { Chlor }\end{array}$ & $\begin{array}{l}\mathrm{DMSO} / \\
\mathrm{EE}\end{array}$ & $\begin{array}{l}\text { DMSO/ } \\
\text { TC }\end{array}$ & PBS/VA & & $\begin{array}{l}\text { DMSO } \\
\text { /Dm }\end{array}$ & $\begin{array}{l}\mathrm{PBS} / \\
\mathrm{LiCO}_{3}\end{array}$ \\
\hline 79 & 088441 & Mtx2 & Metaxin-2 & $4.80 \mathrm{E}-02$ & -1.67 & -1.73 & -1.36 & -1.50 & -1.32 & -1.39 & -1.07 \\
\hline 80 & 008709 & $\operatorname{Prd} \times 6$ & Peroxiredoxin- 6 & $2.70 \mathrm{E}-03$ & -1.04 & 1.04 & -1.12 & -1.14 & $1.56^{* *}$ & -1.00 & 1.09 \\
\hline 81 & P17751 & Tpi1 & Triosephosphate isomerase & $2.00 \mathrm{E}-03$ & 1.03 & 1.02 & -1.13 & -1.12 & 1.21 & 1.05 & 1.14 \\
\hline 82 & P17751 & Tpi1 & Triosephosphate isomerase & $1.30 \mathrm{E}-02$ & 1.14 & 1.03 & -1.10 & -1.11 & 1.14 & 1.10 & 1.19 \\
\hline 83 & P19639 & Gstm3 & Glutathione S-transferase Mu 3 & $2.60 \mathrm{E}-02$ & -1.10 & -1.03 & -1.25 & -1.41 & 1.27 & 1.07 & 1.20 \\
\hline 84 & 035660 & Gstm6 & Glutathione S-transferase Mu 6 & $2.80 \mathrm{E}-02$ & -1.01 & 1.16 & 1.02 & 1.54 & 1.16 & -1.03 & 1.11 \\
\hline 85 & P10648 & Gsta2 & Glutathione S-transferase A2 & $8.30 \mathrm{E}-05$ & 1.21 & 1.46 & 1.40 & -1.32 & $1.54^{* *}$ & 1.01 & 1.17 \\
\hline 86 & P10648 & Gsta2 & Glutathione S-transferase A2 & $1.30 \mathrm{E}-02$ & 1.05 & 1.31 & 1.08 & 1.30 & $1.66^{* *}$ & 1.01 & 1.09 \\
\hline 87 & 088587 & Comt1 & $\begin{array}{l}\text { Isoform Membrane-bound of Catechol O- } \\
\text { methyltransferase }\end{array}$ & $6.20 \mathrm{E}-03$ & -1.17 & -1.13 & -1.15 & -1.27 & -1.28 & 1.04 & 1.05 \\
\hline 88 & Q61171 & $\operatorname{Prdx} 2$ & Peroxiredoxin-2 & $8.40 \mathrm{E}-03$ & -1.01 & -1.08 & $-1.21^{* *}$ & 1.03 & -1.08 & -1.04 & 1.00 \\
\hline 89 & P35700 & $\operatorname{Prdx} 1$ & Peroxiredoxin-1 & $1.60 \mathrm{E}-06$ & 1.04 & 1.04 & -1.05 & $1.25^{* *}$ & $1.18^{* *}$ & 1.02 & 1.06 \\
\hline 90 & P09671 & Sod2 & Superoxide dismutase $[\mathrm{Mn}]$, mitochondrial & 1.10E-02 & -1.09 & -1.14 & -1.02 & 1.17 & -1.01 & -1.10 & -1.04 \\
\hline 91 & P10639 & Txn1 & Thioredoxin & $1.10 \mathrm{E}-03$ & 1.05 & 1.17 & -1.01 & -1.06 & $1.39^{* *}$ & -1.09 & 1.34 \\
\hline
\end{tabular}

${ }^{1}$ P-value from one way ANOVA statistical test between the experimental groups chlorpromazine (Chlor), ethinylestradiol (EE), tetracycline (TC), valproic acid (VA), paraquat $(\mathrm{PQ})$, negative controls $\mathrm{D}$-mannitol $(\mathrm{Dm})$ and $\mathrm{LiCO}_{3}$, with each five biological replicates.

${ }^{2}$ The difference in the standardized abundance of the proteins is expressed as the fold change between the control (C) and the treated groups (T). The fold change is calculated by taking the means of standardized volume values for the protein spot in the corresponding groups $(\mathrm{PBS}=\mathrm{PBS}$ vehicle control, $\mathrm{DMSO}=\mathrm{DMSO}$ vehicle control, $\mathrm{Pq}=$ paraquat), values are calculated as T/C and displayed in the range of +1 to $+\infty$ for increases in expression and calculated as $-C / T$ and displayed in the range of $-\infty$ to -1 for decreased expression.

** Indicates significant fold changes $(P \leq 0.05)$ between the control and the treated group, calculated with a multiple comparison test.

*Indicates significant fold changes $(P \leq 0.1)$ between the control and the treated group, calculated with a multiple comparison test. 
Dankwoord 
Mijn promotietraject was een uitdaging waar geen einde aan leek te komen. Het combineren van een nieuwe baan met het schrijven van een proefschrift was moeilijker dan ik dacht. Door verschillende mensen werd me meermaals gevraagd of mijn proefschrift nu eindelijk klaar was. Ondertussen ben ik eindelijk gekomen aan het laatste hoofdstuk. Een hoofdstuk waarin ik de tijd wil nemen om al deze mensen te bedanken voor interesse, bezorgdheid en steun.

Allereerst wil ik mijn promotores Prof. dr. Edwin Marin, Prof. dr. Jos Kleinjans en copromotores dr. Johan Renes en dr. Joost van Delft bedanken. Edwin, als promotor speelde je een centrale rol tijdens mijn promotietraject. Ik kon steeds bij je binnenlopen om het eender wat. Ook na de zoveelste paniekaanval wist je de rust te bewaren, "alles komt goed" zei je. Even leek het of je daar zelf aan twijfelde, en zei je "de wonderen zijn de wereld nog niet uit". Echte wonderen zijn er (denk ik) niet gebeurd, maar uiteindelijk is alles goed gekomen dankzij jou.

Jos, bedankt voor de kansen die ik heb gekregen. Ik heb veel geleerd van je opbouwende en leerzame commentaren bij het nalezen van mijn schrijfsels.

Johan, dankjewel voor je vertrouwen in mij. Ik kreeg van jou de vrijheid in het opzetten van experimenten, waardoor ik me kon ontwikkelen tot een zelfstandig onderzoeker. Telkens weer moedigde je me aan om door te zetten en met je kritische blik wist je de puntjes op de ' $i$ ' te zetten.

Joost, bedankt voor de revisies van alle hoofdstukken en manuscripten die dit proefschrift naar een hoger niveau hebben gebracht. Jammer dat je vlak voor de eindstreep hebt moeten afhaken. Veel succes met je nieuwe baan!

De beoordelingscommissie bestaande uit Prof. dr. W. H. Lamers, Prof. dr. L. Arckens, Prof. dr. H. van Loveren, dr. S. Olde Damink en Prof dr. H. H. H. W. Schmidt, wil ik graag bedanken voor het kritisch evalueren van mijn proefschrift.

Mijn paranimfen en kamergenoten Freek Bouwman en Antoine Zorenc, we hebben samen een leuke tijd gehad waar ik nog vaak aan terugdenk. We hebben goed kunnen lachen, maar ook konden we elkaar vinden tijdens moeilijkere momenten. Ik ben daarom blij dat jullie ook bij de verdediging naast me zullen staan! Ook kon ik steeds terugvallen op jullie technische expertise. Wanneer mijn gelen of western-blots niet wilden lukken hielpen jullie mee een oplossing te bedenken. Bedankt voor alles!

Ping Wang, no question was too much you were always there for me. I admire you for your passion for science and how you pass this passion on to the rest of the group.

Anja Rosenow, het grootste deel van ons promotietraject hebben we samen doorlopen. Gelukkig was er ook tijd voor ontspanning, tijdens de koffieklets bij de DE-corner konden we elkaars hart luchten. Ook werden er uitstapjes gemaakt naar Aken, Dortmund en Berlijn. Overal was je mijn persoonlijke gids. Bedankt voor de fijne tijd die we samen hebben doorgebracht. 
Ook andere mensen van Humane Biologie wil ik bedanken voor de fijne sfeer en gezelligheid. (Ex-)FunGenners, Janneke, Ronny, Jonathan, Montserrat, Roel, en Egbert, bedankt voor de fijne samenwerking. Ellen, Anneke, Johan J., Yvonne, Joan en Karianna het was steeds gezellig om met jullie in het labo te werken. Madeleen en Bianca, bedankt voor de gezellige babbeltjes! Jos, het is al meermaals gezegd, je bent de vaste waarde van Humane Biologie! Samen met alle andere HB-ers wil ik je bedanken voor alle fijne momenten tijdens de borrels, kerstdiners, vakgroepuitjes, promotiefeestjes, carnaval...

Al was mijn werkplek bij Humane Biologie, ook heb ik ook heel wat tijd doorgebracht bij de mensen van Toxicogenomics.

Dankzij Karen Mathijs leerde ik de muizen leverperfusie. Karen, zonder jou weet ik niet of ik erin geslaagd was om deze techniek te leren, bedankt om jouw ervaringen te delen.

Daneida Lizarraga, thanks for everything I learned from you! I enjoyed our collaboration during the liver perfusions and the experiments we did together.

De transcriptome analyse uit dit proefschrift was nooit tot stand gekomen zonder Karen Brauers en Charly John. Karen bedankt voor je hulp en de tijd die je hebt willen vrijmaken! Charly thanks for your help with the data analysis of the micro-arrays.

Wim van Hof mijn vaste taxi naar de NTC-meetings $(-)$, bedankt voor de fijne ritten en samenwerking.

Een dikke merci aan de mensen die allerlei administratieve zaken voor mij in orde hebben gebracht: Anneloes Melman, René Rijnders, Rob Schoolz en Sandrien Wansink.

Verder wil ik alle andere mensen van Toxicogenomics bedanken voor het warme onthaal en de leuke sfeer in het labo, tijdens meetings, en uitjes!

Een eerste kennismaking met wetenschappelijk onderzoek kreeg ik in het labo van Prof. dr. Jean-paul Noben, tijdens mijn stage aan de universiteit Hasselt. Ruth Daniels, mijn toenmalige stage begeleidster moedigde me aan om te doctoreren. Dankzij haar heb ik die stap durven nemen. Uiteindelijk niet aan de universiteit van Hasselt, maar toch bleef de samenwerking bestaan. Jean-Paul en Erik Royackers zorgden voor de identificatie van verschillende eiwitspotjes, zonder hen had dit proefschrift er vast anders uitgezien.

Tijdens het scannen en analyseren van de DIGE gelen heb ik veel tijd doorgebracht in het labo van klinische chemie. Bianca, Daniëlle, Yves en Anita, bedankt voor jullie hulp en gezelligheid.

Anne Kienhuis, voor mij ben je een echte toxicogenomics-expert. Ik bewonder je enthousiaste en doordachte manier van onderzoek doen. Bedankt voor de fijne samenwerking!

Mijn huidige collega's bij Estée Lauder, bedankt voor jullie interesse en het toeleven naar de eindelijke verdediging.

Familie en vrienden bedankt voor jullie interesse en steun. Eindelijk zien jullie het resultaat van hetgeen waar ik zolang aan heb gewerkt. 
Schrijven is nooit mijn beste kant geweest. Mijn zus Ilse is de talenknobbel van de familie, gelukkig kon ik op haar hulp rekenen bij het nalezen van een aantal manuscripten.

Lieve mama en papa, bedankt voor de steun en kansen die ik van jullie heb gekregen. Steeds kan ik op jullie rekenen, ik kan niet beschrijven hoe dankbaar ik jullie ben voor alles.

Bert, het was voor jou niet makkelijk om samen te zijn met een doctorerende vriendin. Samen trokken we de grens over naar Roermond, waar ons avontuur begon. Roermond, juist tussen ons werk in Eindhoven en Maastricht, daar was over nagedacht. Toch kon je er maar moeilijk aarden, elk weekend was je dan ook opgelucht als we weer richting Lommel reden. Het was dan ook geen toeval dat we snel terug naar onze geboorteplaats verhuisden, dicht bij onze vrienden en familie. Ik had ondertussen een nieuwe baan gevonden, maar dat proefschrift was nog niet af... Je hebt veel geduld moeten hebben maar gelukkig is het proefschrift nu eindelijk af. Ik kijk er naar uit om weer meer tijd met je door te brengen! Bedankt voor alles lieve schat! 
Curriculum Vitae 
Anke Van Summeren werd geboren op 17 augustus 1984 te Lommel, België. In 2002 behaalde ze haar secundair diploma in 'Techniek Wetenschappen' aan de WICO campus Sint-Jozef te Lommel. In datzelfde jaar begon ze aan de bachelor opleiding Chemie (specialisatie Biochemie), aan de Katholieke Hogeschool Limburg (KHLim) te Diepenbeek, België en studeerde af in 2005. Om haar biochemische kennis verder uit te breiden startte ze de master opleiding Industriële Wetenschappen, Biochemie aan de KHLim (in associatie met de Katholieke Universiteit Leuven) te Diepenbeek. Voor haar master thesis deed ze 4 maanden stage bij the Biomedisch Onderzoeksinstituut van de Universiteit Hasselt. Na het beëndigen van haar thesis 'Kwantitatieve analyse van differentiële expressie in het hersenproteoom bij het EAE-proefdiermodel voor multiple sclerose', studeerde ze af in 2007. Daarna werkte ze als project assistent bij IMEC te Leuven, België waar ze onderzoek deed naar biosensoren op basis van nanoparticle films. In april 2008 startte ze haar promotie onderzoek aan de Universiteit Maastricht bij de afdeling Toxicogenomics in samenwerking met de afdeling Humane Biologie. Sinds maart 2012 werkt ze als project engineer op de onderzoeksafdeling van Estée Lauder te Oevel, België.

Anke Van Summeren was born on August 17th 1984 in Lommel, Belgium. In 2002, she graduated from her secundairy education in the discipline 'Technical Sciences' at the WICO campus Sint-Jozef in Lommel. In the same year, she started the bachelor study Chemistry, with specialization in Biochemistry, at the KHLim in Diepenbeek, Belgium and graduated in 2005. For more in-depth knowledge on biochemistry she completed her education with the master Industrial Sciences in Biochemistry at the KHLim (in association with the Catholic University of Leuven) in Diepenbeek. For her thesis she performed a 4-month internship at the Biomedical Research Institute of Hasselt University. She finished her thesis 'Quantitative analysis of differential expression in the brain proteome of the EAE laboratory animal model for multiple sclerosis', and graduated in 2007. After her graduation she joined the company IMEC in Leuven, Belgium as a project assistant were she performed research on biosensing based on the use of gold nanoparticle films. In April 2008 she started as a PhD student at Maastricht University at the department of Toxicogenomics in cooperation with the department of Human Biology. From March 2012 onwards, Anke has been working as a project engineer at the Basic Science Research department of Estée Lauder in Oevel, Belgium. 
List of publications 


\section{FULL PAPERS}

Van Summeren A., Renes J., van Delft J. H. M., Kleinjans J. C. S., Mariman E. C. M. Proteomics in the search for mechanisms and biomarkers of drug-induced hepatotoxicity. Toxicology in vitro : an international journal published in association with BIBRA 26, 373-385, (2012).

Van Summeren A., Renes J., Bouwman F. G., Noben J. P., van Delft J. H. M., Kleinjans J. C. S., and Mariman E. C. M. Proteomics investigations of drug-Induced hepatotoxicity in HepG2 cells. Toxicol Sci 120, 109-122 (2011).

Van Summeren A., Renes J., Lizarraga D., Bouwman F. G., Noben J. P., van Delft J. H. M., Kleinjans J. C. S., and Mariman E. C. M. Screening for drug-induced hepatotoxicity in primary mouse hepatocytes using acetaminophen, amiodarone, and cyclosporin a as model compounds: an omics-guided approach. Omics : a journal of integrative biology 17, 71-83 (2013).

Van Summeren A., Renes J., Bouwman F. G., Noben J. P., van Delft J. H. M., Kleinjans J. C. S., and Mariman E. C. M. The secretome of primary mouse hepatocytes in drug-Induced hepatotoxicity Screening. Submitted.

Van Summeren A., Lizarraga D., Renes J., Bouwman F. G., Noben J. P., van Delft J. H. M., Kleinjans J. C. S., and Mariman E. C. M. Conserved hepatotoxic responses in HepG2 and primary mouse hepatocytes. In preparation.

Van Summeren A., Kienhuis A., Renes J., Bouwman F. G., Speksnijder E., Noben J. P., van Delft J. H., Kleinjans J. C., and Mariman E. .C. M. In vitro to in vivo comparison of cyclosporin Ainduced protein expression profiles. Submitted.

Van Summeren A., Renes J., Bouwman F. G., Noben J. P., van Delft J. H. M., Kleinjans J. C. S., and Mariman E. C. M. Analysis of protein expression patterns induced by classifying hepatotoxicants in primary mouse hepatocytes. In preparation.

Van den Hof W. F. P. M., Ruiz-Aracama A., Van Summeren A, Jennen D. G. J., Gaj S., Coonen M., Brauers K., Wodzig W. K. W. H., Kleinjans J. C. S.,. van Delft J. H. M. Integrating multiple omics to unravel mechanisms of cyclosporin A induced hepatotoxicity. Submitted.

Jiang J., Briedé J. J., Jennen D. G. J., Van Summeren A., Brauers K., Kleinjans J. C. S, de Kok T. M. C. M. Non-cytotoxic doses of acetaminophen results in disrupted expression of mitochondrial electron transport chain related genes explaining the associated increased mitochondrial ROS formation in hepatic cells. Submitted. 
Bonroy K., Jans H., Van de Broek B., Jans K., Reekmans G., Van Summeren A., Van Meerbergen B., Huang C., Stakenborg T., Trekker J., Willems M., Bartic C, Verhaegen K., Borghs G. Functionalized Inorganic Nanostructures For Biomedical Applications. NSTI Nanotech 2008 Technical Proceedings - Life Sciences, Medicine, and Bio Materials: Vol. 2. Nanotech 2008. Boston, USA, 2008 1-5 June; 262 -265.

\section{LETTERS}

Van Summeren A., Renes J., Mariman E. C. M., Kleinjans J. C. S. and van Delft J. H. M. Response to Pathophysiological Relevance of Proteomics Investigations of Drug-Induced Hepatotoxicity in HepG2 Cells. Toxicol Sci 121, 431-433 (2011).

\section{ABSTRACTS}

Van Summeren A., Renes J., Bouwman F. G., Noben J. P., van Delft J. H. M., Kleinjans J. C. S., and Mariman E. C. M. Proteomics Investigations of Drug-Induced Hepatotoxicity in HepG2 Cells and primary Mice Hepatocytes. Poster presentation at Proteomic Forum, April 2011 Berlin, Germany.

Van Summeren A., Renes J., Bouwman F. G., Noben J. P., van Delft J. H. M., Kleinjans J. C. S., and Mariman E. C. M. Proteomics Investigations of Drug-Induced Hepatotoxicity in HepG2 Cells and primary Mice Hepatocytes. Oral presentation at conference of The Royal Flemish Chemical Society on 'Proteomics and the world of tomorrow', December 2010, Antwerp, Belgium.

Van Summeren A., Renes J., Bouwman F. G., Noben J. P., van Delft J. H. M., Kleinjans J. C. S., and Mariman E. C. M. Proteomics Investigations of Drug-Induced Hepatotoxicity in HepG2 Cells. Poster presentation at the PhD meeting of the Dutch Society of Toxicology and Annual Meeting of the NVT, June 2010, Zeist, The Netherlands.

Van Summeren A., Renes J., van Delft J. H. M., Kleinjans J. C. S., and Mariman E. C. M. Proteomics investigations towards mechanisms and Biomarkers for hepatotoxicity. Oral presentation at the Annual meeting of the Netherlands Toxicogenomics Centre (NTC), January 2010, Amsterdam, The Netherlands.

Van Summeren A., Renes J., Bouwman F. G., Noben J. P., van Delft J. H. M., Kleinjans J. C. S, and Mariman E. C. M. Proteomics Investigations of Drug-Induced Hepatotoxicity in HepG2 Cells. Poster presentation at the Annual Nutrim day, December 2009, Maastricht, The Netherlands. 
Van Summeren A., Renes J., van Delft J. H. M., Kleinjans J. C. S., and Mariman E. C. M. Proteomics investigations towards mechanisms and Biomarkers for hepatotoxicity. Poster presentation at the PhD meeting of the Dutch Society of Toxicology and Annual Meeting of the NVT, June 2009, Wageningen, The Netherlands.

Van Summeren A., Renes J., van Delft J. H. M., Kleinjans J. C. S., and Mariman E. C. M. Proteomics investigations towards mechanisms and Biomarkers for hepatotoxicity. Poster presentation at the Annual Nutrim day, November 2008, Maastricht, The Netherlands. 